\title{
Kobayashi-Hitchin correspondence for tame harmonic bundles II
}

\author{
TAKURO MOCHIZUKI
}

\begin{abstract}
Let $X$ be a smooth irreducible projective complex variety with an ample line bundle $L$, and $D$ be a simple normal crossing hypersurface. We establish the KobayashiHitchin correspondence between tame harmonic bundles on $X-D$ and $\mu_{L}$-stable parabolic $\lambda$-flat bundles with trivial characteristic numbers on $(X, D)$. In particular, we obtain the quasiprojective version of the Corlette-Simpson correspondence between flat bundles and Higgs bundles.
\end{abstract}

$14 \mathrm{~J} 60 ; 53 \mathrm{C} 07$

\section{Introduction}

\subsection{Main results}

We explain the main results of this paper. We do not review the history or the background on the study of Kobayashi-Hitchin correspondence and harmonic bundles, for which we refer to Lübke and Teleman [11], Simpson [19; 20] or Mochizuki [14], for example. The notion of regular filtered $\lambda$-flat bundle and parabolic $\lambda$-flat bundle are explained in Section 2.1. (See also Sections 3.1-3.2 of [14]. But note that we use slightly different notation and terminology.) Since they are equivalent, we will not make a distinction between them in this introduction. The notion of filtered local system is explained in Section 6.

1.1.1 Kobayashi-Hitchin Correspondence Let $X$ be a smooth irreducible complex projective variety with an ample line bundle $L$. Let $D$ be a simple normal crossing hypersurface of $X$. Our main purpose is to show the following theorem.

Theorem 1.1 (Theorem 5.16, Proposition 2.55, Proposition 2.56) Let $\left(\mathbf{E}_{*}, \mathbb{D}^{\lambda}\right)$ be a regular filtered $\lambda$-flat bundle on $(X, D)$. We put $E:=\mathbf{E}_{\mid X-D}$. Then the following conditions are equivalent.

- $\left(\mathbf{E}_{*}, \mathbb{D}^{\lambda}\right)$ is $\mu_{L}$-polystable with trivial characteristic numbers.

- There exists a pluri-harmonic metric $h$ of $\left(E, \mathbb{D}^{\lambda}\right)$ adapted to the parabolic structure.

Such a metric is unique up to obvious ambiguity. 
Remark 1.2 Theorem 1.1 in the case $\lambda=0$ has already been proven in our previous paper [14]. Hence, we restrict ourselves to the case $\lambda \neq 0$ in this paper.

Corollary 1.3 (Corollary 5.18) Let $\mathcal{C}_{\lambda}^{\text {poly }}$ denote the category of $\mu_{L}$-polystable regular filtered $\lambda$-flat bundles on $(X, D)$ with trivial characteristic numbers. Then we have the natural equivalence of the categories $\Xi_{\lambda_{1}, \lambda_{2}}: \mathcal{C}_{\lambda_{1}}^{\text {poly }} \simeq \mathcal{C}_{\lambda_{2}}^{\text {poly }}$ for any $\lambda_{i} \in \mathbf{C}$ $(i=1,2)$. The equivalence preserves tensor products, direct sums and duals.

Remark 1.4 Let $\lambda_{i} \in \mathbf{C}^{*}(i=1,2)$. A $\lambda_{2}$-connection $\mathbb{D}^{\lambda_{2}}=d^{\prime \prime}+\left(\lambda_{2} / \lambda_{1}\right) \cdot d^{\prime}$ is induced by a $\lambda_{1}$-connection $\mathbb{D}^{\lambda_{1}}=d^{\prime \prime}+d^{\prime}$, which gives the obvious functor Obv: $\mathcal{C}_{\lambda_{1}}^{\text {poly }} \longrightarrow \mathcal{C}_{\lambda_{2}}^{\text {poly }}$. Note that this is not the same as the above functor $\Xi_{\lambda_{1}, \lambda_{2}}$.

In particular, we obtain a generalization of the Corlette-Simpson correspondence between flat bundles and Higgs bundles in the so-called nonabelian Hodge theory.

Corollary 1.5 We have an equivalence of the following two categories:

- The category of $\mu_{L}$-polystable regular filtered Higgs bundles on $(X, D)$ with trivial characteristic numbers.

- The category of $\mu_{L}$-polystable regular filtered flat bundles on $(X, D)$ with trivial characteristic numbers.

Remark 1.6 C Simpson [18] established these results in the case $\operatorname{dim} X=1$. O Biquard [1] obtained the correspondence in the case that $D$ is smooth.

\subsubsection{Bogomolov-Gieseker inequality and a formula for the characteristic num-} bers Let $X, L$ and $D$ be as above.

Theorem 1.7 (Corollary 3.20) Let $\left(\mathbf{E}_{*}, \mathbb{D}^{\lambda}\right)$ be a $\mu_{L}$-stable regular filtered $\lambda$-flat bundle on $(X, D)$. Then the following inequality holds for the parabolic characteristic numbers for $\mathbf{E}_{*}$ :

$$
\int_{X} \operatorname{par-ch}{ }_{2, L}\left(\mathbf{E}_{*}\right) \leq \frac{\int_{X} \operatorname{par}^{-c_{1, L}^{2}}\left(\mathbf{E}_{*}\right)}{2 \operatorname{rank} E} .
$$

It is a generalization of the so-called Bogomolov-Gieseker inequality.

In the case $\lambda \neq 0$, there exist some formulas to express $\int_{X}$ par-ch ${ }_{2, L}\left(\mathbf{E}_{*}\right)$ in terms of the data at $D$, which are valid for any parabolic $\lambda$-flat bundles. One of them is comprehensible from the viewpoint of the correspondence between regular filtered $\lambda$-flat sheaves and filtered local systems. Let $\left(\mathbf{E}_{*}, \mathbb{D}^{\lambda}\right)$ be a regular filtered $\lambda$-flat sheaf 
on $(X, D)$. As is explained in Remark 1.4, we have the obvious correspondence of a flat $\lambda$-connection $\mathbb{D}^{\lambda}=d^{\prime \prime}+d^{\prime}(\lambda \neq 0)$ and a flat connection $\mathbb{D}^{\lambda f}=d^{\prime \prime}+\lambda^{-1} d^{\prime}$. In particular, we obtain a local system $\mathcal{L}$ on $X-D$ from the flat bundle $\left(\mathbf{E}_{*}, \mathbb{D}^{\lambda, f}\right)_{\mid X-D}$. Moreover, the parabolic structure of $\left(\mathbf{E}_{*}, \mathbb{D}^{\lambda}\right)$ induces the filtered structure of $\mathcal{L}$, and we have the more refined claims as in the following proposition.

Proposition 1.8 (Corollary 6.5 and Corollary 6.7) Let $\widetilde{\mathcal{C}}(X, D)$ denote the category of filtered local systems on $(X, D)$, and let $\mathcal{C}_{\lambda}^{\text {sat }}(X, D)$ denote the category of saturated regular filtered $\lambda$-flat sheaves on $(X, D)$ for $\lambda \neq 0$. Then we have an equivalence of categories $\Phi_{\lambda}: \tilde{\mathcal{C}}(X, D) \longrightarrow \mathcal{C}_{\lambda}^{\text {sat }}(X, D)$ such that par-c ${ }_{1}\left(\Phi_{\lambda}\left(\mathcal{L}_{*}\right)\right)=\operatorname{par} \mathrm{c}_{1}\left(\mathcal{L}_{*}\right)$ and $\int_{X}$ par-ch ${ }_{2, L}\left(\Phi_{\lambda}\left(\mathcal{L}_{*}\right)\right)=\int_{X}$ par-ch ${ }_{2, L}\left(\mathcal{L}_{*}\right)$. The functor $\Phi_{\lambda}$ preserves the $\mu_{L}-$ stability.

Let us also describe the formula $\int_{X}$ par-ch ${ }_{2, L}\left(\Phi\left(\mathcal{L}_{*}\right)\right)=\int_{X}$ par-ch ${ }_{2, L}\left(\mathcal{L}_{*}\right)$ in terms of the $\mathbf{c}$-truncation $\left(\mathbf{c} E_{*}, \mathbb{D}^{\lambda}\right)$ of saturated regular filtered $\lambda$-flat bundle $\Phi_{\lambda}\left(\mathcal{L}_{*}\right)$. For simplicity, we assume $\operatorname{dim} X=2$.

(1) $\int_{X} \operatorname{par}-\operatorname{ch}_{2}\left(\mathrm{c} E_{*}\right)$

$=\frac{1}{2} \sum_{i \in S} \sum_{u \in \mathcal{K} \mathcal{M S}\left({ }_{\mathrm{c}} E_{*}, i\right)}\left(\operatorname{Re}\left(\lambda^{-1} \alpha\right)+a\right)^{2} \cdot r(i, u) \cdot\left[D_{i}\right]^{2}$

$+\frac{1}{2} \sum_{i \in S} \sum_{\substack{j \neq i \\ P \in D_{i} \cap D_{j}}} \sum_{\left(u_{i}, u_{j}\right) \in \mathcal{K} \mathcal{M S}\left(\mathrm{c}_{\mathrm{c}} E_{*}, P\right)}\left(\operatorname{Re}\left(\lambda^{-1} \alpha_{i}\right)+a_{i}\right)\left(\operatorname{Re}\left(\lambda^{-1} \alpha_{j}\right)+a_{j}\right) \cdot r\left(P, u_{i}, u_{j}\right)$.

Here, $u=(a, \alpha), u_{i}=\left(a_{i}, \alpha_{i}\right)$ and $u_{j}=\left(a_{j}, \alpha_{j}\right)$ denote elements of the KMSspectrum of $\left({ }_{\mathbf{c}} E, \mathbb{D}^{\lambda}\right)$. We put $r(i, u):=\operatorname{rank}^{i} \mathrm{Gr}_{u}^{F, \mathbb{E}}\left({ }_{\mathbf{c}} E\right)$ for $u \in \mathcal{K} \mathcal{M S}\left({ }_{\mathbf{c}} E_{*}, i\right)$, and $r\left(P, u_{i}, u_{j}\right):=\operatorname{rank}^{P} \operatorname{Gr}_{\left(u_{i}, u_{j}\right)}^{F, \mathbb{E}}\left(E_{\mid P}\right)$ for $\left(u_{i}, u_{j}\right) \in \mathcal{K} \mathcal{M S}\left({ }_{\mathbf{c}} E, P\right)$ and $P \in D_{i} \cap D_{j}$. And, $\left[D_{i}\right]^{2}$ denote the self-intersection number of $D_{i}$.

Remark 1.9 We also have some other formulas for $\int_{X}$ par-ch ${ }_{2}\left(\mathbf{c} E_{*}\right)$ (Proposition 3.22) or some vanishings for the data of $\left({ }_{\mathbf{c}} E_{*}, \mathbb{D}^{\lambda}\right)$ at $D$ (Corollary 3.20 and Proposition $3.22)$.

Remark 1.10 From Theorem 1.7 and Proposition 1.8, we obtain the BogomolovGieseker inequality for $\mu_{L}$-stable filtered local systems (Corollary 6.8). This kind of inequality is discussed by Simpson [21]. 


\subsubsection{Vanishing of the characteristic numbers and existence of the Corlette-} Jost-Zuo metric Due to Proposition 1.8, we obtain the vanishings $\operatorname{par}-\operatorname{deg}_{L}\left(\mathbf{E}_{*}\right)=$ $\int_{X} \operatorname{par}^{-c_{2, L}}\left(\mathbf{E}_{*}\right)=0$, when $\left(\mathbf{E}_{*}, \nabla\right)$ corresponds to a filtered local system whose parabolic structure is trivial, in other words, $\operatorname{Re}(\alpha)+a=0$ is satisfied for any element of the KMS-spectrum $u=(a, \alpha) \in \mathcal{K} \mathcal{M S}(i)$ and for any $i \in S$. We can apply such a consideration to the canonical prolongation of a flat bundle due to P Deligne [3]. Let $(E, \nabla)$ be a flat bundle on $X-D$. Deligne showed [3, Section II.5] that there uniquely exists a holomorphic vector bundle $\widetilde{E}$ on $X$ satisfying (i) $\widetilde{E}_{\mid X-D}=E$, (ii) $\nabla \widetilde{E} \subset \widetilde{E} \otimes \Omega_{X}^{1,0}(\log D)$, (iii) the real parts of any eigenvalues of $\operatorname{Res}_{i}(\nabla)$ are contained in $[0,1[$. (Note that he also studied the case in which $D$ is not necessarily normal crossing. See also the nice textbook by Hotta, Takeuchi and Tanisaki [8].) In that case, we have the naturally defined parabolic structure $\mathbf{F}$ for which $\operatorname{Re}(\alpha)+a=0$ is satisfied for any element of the KMS-spectrum $(a, \alpha)$. Hence, we obtain the vanishing $\operatorname{par}^{-d_{e}}{ }_{L}(\widetilde{E}, \mathbf{F})=\int_{X} \operatorname{par}^{-c_{2, L}}(\widetilde{E}, \mathbf{F})=0$.

This vanishing is significant to understand the existence theorem for the Corlette-JostZuo metric from the viewpoint of Kobayashi-Hitchin correspondence. Recall the existence of a tame pure imaginary pluri-harmonic metric for a semisimple flat bundle $(E, \nabla)$ on $X-D$, which we call the Corlette-Jost-Zuo metric. (See Corlette [2] for the case $D=\varnothing$ and Jost and Zuo [10] for the general case. See also Mochizuki [15].) Since semisimplicity of $(E, \nabla)$ is equivalent to the $\mu_{L}$-polystability of $(\widetilde{E}, \mathbf{F}, \nabla)$ (see Sabbah [16], for example), we can derive the existence of the Corlette-Jost-Zuo metric from Theorem 1.1 and the above vanishing of the characteristic numbers. (See Section 5.3.3.)

\subsection{Methods and difficulty}

1.2.1 Perturbation of parabolic structure Let us explain our basic strategy in [14] and this paper. See also Section 1.2.2 of [14].

Simpson [17] showed a very nice result on the existence of Hermitian-Einstein metrics for a Higgs bundle on open manifolds, which can be generalized for flat $\lambda$-connections for any $\lambda$, as he mentioned in [18]. To apply it, however, we need to construct an initial metric whose pseudo-curvature satisfies some finiteness condition. In the one dimensional case, a construction was done by Simpson himself. The nilpotent part of the residue on the graded pieces made it more complicated than that for ordinary parabolic bundles. If $D$ is smooth, it was generalized by Biquard. However, in the normal crossing case, it is difficult to generalize their construction without any assumption on compatibility of the nilpotent parts of the residues.

To overcome it, we introduced the method of $\epsilon$-perturbation in [14]. Let us briefly recall it for flat $\lambda$-connections. (See Section 2.1.6 for more details and precise.) 
Let $X$ be a smooth irreducible projective surface, and let $D$ be a simple normal crossing divisor of $X$. Let $\left(E, \mathbf{F}, \mathbb{D}^{\lambda}\right)$ be a parabolic $\lambda$-flat bundle on $(X, D)$. On each graded piece ${ }^{i} \mathrm{Gr}_{a}^{F}(E)$, we obtain the filtration by vector subbundles such that it gives the weight filtration of the nilpotent part of $\operatorname{Gr}_{a}^{F}\left(\operatorname{Res}_{i}\left(\mathbb{D}^{\lambda}\right)\right)$ at the generic point of $D_{i}$. Mixing it to the parabolic filtration, for any small $\epsilon>0$, we take an $\epsilon-$ perturbation $\mathbf{F}^{(\epsilon)}$ of the parabolic structure so that $\left(E, \mathbf{F}^{(\epsilon)}, \mathbb{D}^{\lambda}\right)$ is graded semisimple, ie, the residues are essentially semisimple. A construction of an initial metric for $\left(E, \mathbf{F}^{(\epsilon)}, \mathbb{D}^{\lambda}\right)$ is not difficult, which can be done in a rather naive way (Section 3). Hence, if $\left(E, \mathbf{F}^{(\epsilon)}, \mathbb{D}^{\lambda}\right)$ is $\mu_{L}$-stable, we obtain the existence of a Hermitian-Einstein metric $h_{\mathrm{HE}}^{(\epsilon)}$ of $\left(E_{\mid X-D}, \mathbb{D}^{\lambda}\right)$ which is adapted to $\mathbf{F}^{(\epsilon)}$ for $\epsilon>0$. In particular, we obtain the Bogomolov-Gieseker inequality for $\left(E, \mathbf{F}^{(\epsilon)}, \mathbb{D}^{\lambda}\right)$.

Then we can easily derive the Bogomolov-Gieseker inequality (Theorem 1.7) since the characteristic numbers continuously depend on the parabolic weights. As for the existence of a pluri-harmonic metric (Theorem 1.1), we need much more work. Ideally, the $\operatorname{limit}_{\lim _{\epsilon \rightarrow 0}} h_{\mathrm{HE}}^{(\epsilon)}$ should give the desired pluri-harmonic metric for the given parabolic $\lambda$-flat bundle $\left(E, \mathbf{F}, \mathbb{D}^{\lambda}\right)$. However, it is not easy to show such a convergence. That is the main problem which we have to overcome in this paper.

Note that we also obtain formulas like (1) by using the method of $\epsilon$-perturbation.

1.2.2 Difficulty In [14], we gave an argument to deal with such a convergence problem for the case $\lambda=0$. The argument doesn't work in the case $\lambda \neq 0$. Let us explain the difference heuristically and imprecisely in the case $\lambda=1$. Since we have $\operatorname{par}^{-\operatorname{deg}_{L}}\left(E, \mathbf{F}^{(\epsilon)}\right)=0$, the metrics $h_{\mathrm{HE}}^{(\epsilon)}$ give harmonic metrics in this case. Recall that a harmonic metric can be regarded as a harmonic map, at least locally, and that we know a well established argument for the convergence of a sequence of harmonic maps if their energies are dominated. (See Eells and Sampson [6].) In our case, the energies of $h_{\mathrm{HE}}^{(\epsilon)}$ over $X-D$ are not finite, in general. Even if we consider the energies over a compact subset $Z \subset X-D$, it is not clear how to derive an estimate uniformly in $\epsilon$.

If $\lambda=0$, the Higgs field is fixed for this convergence problem. Although the metrics are varied, the eigenvalues of the Higgs field are fixed. Hence, we can derive the estimate of the local $L^{2}$-norms of the Higgs field with respect to the varied metrics, independently from $\epsilon$. Since such $L^{2}$-norms play the role of the energies, the local convergence can be easily shown in the case $\lambda=0$, although we need some more consideration for the global convergence. On the contrary, even the local convergence is not easy to show in the case $\lambda \neq 0$.

1.2.3 Convergences Our argument to attack this convergence problem consists of three steps, which is a variant of the classical one due to S K Donaldson in $[4 ; 5]$. 
Step 1 We study a similar convergence problem in the curve case where the KobayashiHitchin correspondence was established and well understood by the work of Simpson [18]. Let $C$ be a smooth projective curve, and let $D$ be a divisor of $C$. Let $\left(E_{C}, \mathbf{F}_{C}, \mathbb{D}_{C}^{\lambda}\right)$ be a $\lambda$-flat stable parabolic bundle on $(C, D)$, and let $\mathbf{F}_{C}^{(\epsilon)}$ be $\epsilon-$ perturbations. Note $\operatorname{det}\left(E_{C}, \mathbf{F}_{C}, \mathbb{D}_{C}^{\lambda}\right)=\operatorname{det}\left(E_{C}, \mathbf{F}_{C}^{(\epsilon)}, \mathbb{D}_{C}^{\lambda}\right)$. We can take a sequence of harmonic metrics $h_{C}^{(\epsilon)}$ for $\left(E_{C}, \mathbf{F}_{C}^{(\epsilon)}, \mathbb{D}_{C}^{\lambda}\right)(\epsilon \geq 0)$ such that $\operatorname{det} h_{C}^{(\epsilon)}=\operatorname{det} h_{C}^{(0)}$, according to the result of Simpson. We will show that the sequence $\left\{h_{C}^{(\epsilon)} \mid \epsilon>0\right\}$ converges to $h_{C}^{(0)}$.

This is not so easy as it looks. We argue as follows. Let $h_{i n}^{(\epsilon)}(\epsilon>0)$ be initial metrics for $\left(E_{C}, \mathbf{F}_{C}^{(\epsilon)}, \mathbb{D}_{C}^{\lambda}\right)$, and let $s^{(\epsilon)}$ be the endomorphism determined by $h_{C}^{(\epsilon)}=h_{i n}^{(\epsilon)} \cdot s^{(\epsilon)}$. We can show the following relations:

$$
\begin{gathered}
M\left(h_{i n}^{(\epsilon)}, h_{C}^{(\epsilon)}\right) \leq 0, \quad\left|\log s^{(\epsilon)}\right|_{h_{i n}^{(\epsilon)}} \leq C_{1, \epsilon}+C_{2, \epsilon} \cdot M\left(h_{i n}^{(\epsilon)}, h_{C}^{(\epsilon)}\right), \\
\left\|\mathbb{D}^{\lambda} s^{(\epsilon)}\right\|_{L^{2}, h_{i n}^{(\epsilon)}, \omega_{\epsilon}}^{2} \leq \int\left|\operatorname{tr}\left(s^{(\epsilon)} \cdot G\left(h_{i n}^{(\epsilon)}\right)\right)\right| \operatorname{dvol}_{\omega_{\epsilon}}
\end{gathered}
$$

Here, $M\left(h_{i n}^{(\epsilon)}, h_{C}^{(\epsilon)}\right)$ denote the Donaldson functionals, and $\omega_{\epsilon}$ denote appropriate metrics of $C-D$. (See Lemma 2.50 for the first, Proposition 2.41 for the second, and Lemma 4.14 for the third.)

Moreover, we show that $C_{i, \epsilon}$ can be taken independently from $\epsilon$ for some $\omega_{\epsilon}$ (Proposition 2.49), and we can construct appropriate family of initial metrics $h_{i n}^{(\epsilon)}$ such that $G\left(h_{i n}^{(\epsilon)}\right)$ are uniformly bounded with respect to $\omega_{\epsilon}$ and $h_{i n}^{(\epsilon)}$ (Sections 4.2-4.4). Then we obtain the $L_{1}^{2}$-boundedness of the family $\left\{s^{(\epsilon)},\left(s^{(\epsilon)}\right)^{-1} \mid \epsilon>0\right\}$. Then by using a standard bootstrapping argument, we can show that the sequence $\left\{s^{(\epsilon)}\right\}$ is convergent to the identity in the $C^{\infty}$-sense, ie, $\left\{h_{C}^{(\epsilon)}\right\}$ is convergent to $h^{(0)}$ (Proposition 4.1).

Step 2 To argue the convergence of $\left\{h_{\mathrm{HE}}^{(\epsilon)}\right\}$ on $X-D$, we consider the convergence of their restrictions to almost every ample curves in $X$. Note the Mehta-Ramanathan type theorem for regular parabolic $\lambda$-flat bundles (Proposition 2.21). Under the setting of Step 1, it is rephrased as follows. We consider hermitian metrics $\widetilde{h}_{C}^{(\epsilon)}:=h_{C}^{(\epsilon)} \cdot \widetilde{s}^{(\epsilon)}$ for $\epsilon>0$, with the following properties:

- $\operatorname{det} \tilde{h}_{C}^{(\epsilon)}=\operatorname{det} h_{C}^{(\epsilon)}$.

- $\int\left|G\left(\tilde{h}_{C}^{(\epsilon)}\right)\right|^{2} \longrightarrow 0$.

- $\left\|\mathbb{D}^{\lambda} \widetilde{s}^{(\epsilon)}\right\|^{2}<\infty$. (We do not need uniform bound.)

Then we can show that $\left\{\widetilde{h}_{C}^{(\epsilon)}\right\}$ is convergent to $h_{C}^{(0)}$. (See Section 5.1 for more precise claims.) 
Step 3 Let $h_{C}$ be a harmonic metric of $\left(E, \mathbf{F}, \mathbb{D}^{\lambda}\right)_{\mid C}$. Applying the result in Step 2 to $h_{H E \mid C}^{(\epsilon)}=\widetilde{h}_{C}^{(\epsilon)}$, we can show that $\left\{h_{H E \mid C}^{(\epsilon)}\right\}$ is convergent to $h_{C}$ almost everywhere on $C$ for almost every very ample $C \subset X$. Therefore, we obtain a metric $h_{\mathcal{V}}$ defined almost everywhere on $X-D$ such that $h_{\mathcal{V} \mid C}=h_{C}$ almost everywhere on $C$ for almost every curve $C \subset X$. With some more additional argument, we can show that $h_{\mathcal{V}}$ gives the desired pluri-harmonic metric, indeed (Section 5.2).

Remark 1.11 Perhaps, the argument of this paper may be applicable in the Higgs case, to show the existence of a pluri-harmonic metric. However, we remark that the argument for a convergence given in [14] can be applied in a wider range. In fact, we used it to discuss the convergence of a family of harmonic bundles induced by the constant multiplication of Higgs fields.

Acknowledgements This paper is a result of an effort to understand the works of C Simpson, in particular, [17; 19]. I am grateful to N Budur, H Konno, D Panov and C Sevenheck for some discussions. I thank A Ishii and Y Tsuchimoto for their constant encouragement. I express my gratitude to the anonymous referee for his valuable comments. Special thanks go to C Sabbah and K Vilonen.

I am grateful to the colleagues of Department of Mathematics and Research Institute for Mathematical Sciences at Kyoto University for their cooperation. I wrote the first version of this paper during my stay at Max-Planck Institute for Mathematics. I acknowledge their excellent hospitality and support. This work was partly supported by a grant from Ministry of Education, Culture, Sports, Science and Technology.

\section{Preliminaries}

\subsection{Generality of regular filtered $\lambda$-flat sheaves in complex geometry}

We give a generality of regular filtered $\lambda$-flat sheaves in complex geometry. Recall that the notions of parabolic bundle, filtered bundle and their characteristic numbers are explained also in Sections 3.1-3.2 of [14]. We will use the notation there.

\subsubsection{Regular c-parabolic $\lambda$-flat sheaf and regular filtered $\lambda$-flat sheaf}

$\lambda$-connection Let $Y$ be a complex manifold, and let $\mathcal{E}$ be an $\mathcal{O}_{Y}$-module. Recall that a $\lambda$-connection of $\mathcal{E}$ is defined to be a linear map $\mathbb{D}^{\lambda}: \mathcal{E} \longrightarrow \mathcal{E} \otimes \Omega_{Y}^{1,0}$ satisfying the twisted Leibniz rule $\mathbb{D}^{\lambda}(f \cdot s)=f \cdot \mathbb{D}^{\lambda}(s)+\lambda \cdot d_{Y}(f) \cdot s$, where $f$ and $s$ denote holomorphic sections of $\mathcal{O}_{Y}$ and $\mathcal{E}$ respectively. The maps $\mathbb{D}^{\lambda}: \mathcal{E} \otimes \Omega^{p, 0} \longrightarrow$ $\mathcal{E} \otimes \Omega^{p+1,0}$ are induced. If $\mathbb{D}^{\lambda} \circ \mathbb{D}^{\lambda}=0$ is satisfied, it is called flat. 
Regular c-parabolic $\lambda$-flat sheaf Let $X$ be a complex manifold, and let $D$ be a simple normal crossing divisor with the irreducible decomposition $D=\bigcup_{i \in S} D_{i}$. Let $\mathbf{c} \in \mathbf{R}^{S}$. The $i$-th component of $\mathbf{c}$ is denoted by $c_{i}$. Let $\mathcal{E}_{*}=\left(\mathcal{E},\left\{{ }^{i} \mathcal{F} \mid i \in S\right\}\right)$ be a $\mathbf{c}-$ parabolic sheaf on $(X, D)$. Namely, $\mathcal{E}$ is a torsion-free $\mathcal{O}_{X}$-coherent sheaf, and ${ }^{i} \mathcal{F}$ are increasing filtrations of $\mathcal{E}$ indexed by $\left.] c_{i}-1, c_{i}\right]$ such that (i) ${ }^{i} \mathcal{F}_{a}(\mathcal{E}) \supset \mathcal{E} \otimes \mathcal{O}_{X}\left(-D_{i}\right)$, (ii) ${ }^{i} \mathcal{F}_{a}(\mathcal{E})=\bigcap_{a<b}{ }^{i} \mathcal{F}_{b}(\mathcal{E})$, (iii) the sets $\operatorname{Par}\left(\mathcal{E}_{*}, i\right):=\left\{a \mid{ }^{i} \operatorname{Gr}_{a}^{\mathcal{F}}(\mathcal{E}) \neq 0\right\}$ are finite, where ${ }^{i} \operatorname{Gr}_{a}^{\mathcal{F}}(\mathcal{E}):={ }^{i} \mathcal{F}_{a}(\mathcal{E}) /{ }^{i} \mathcal{F}_{<a}(\mathcal{E})$. (See Section 3.1 of [14] for more details on c-parabolic sheaf.)

A flat logarithmic $\lambda$-connection of $\mathcal{E}_{*}$ is defined to be a map $\mathbb{D}^{\lambda}: \mathcal{E} \longrightarrow \mathcal{E} \otimes \Omega^{1,0}(\log D)$ satisfying the same twisted Leibniz rule as above, the flatness $\mathbb{D}^{\lambda} \circ \mathbb{D}^{\lambda}=0$ and $\mathbb{D}^{\lambda}\left({ }^{i} \mathcal{F}_{a}\right) \subset{ }^{i} \mathcal{F}_{a} \otimes \Omega^{1,0}(\log D)$. Such a tuple $\left(\mathcal{E}_{*}, \mathbb{D}^{\lambda}\right)$ will be called a regular parabolic $\lambda$-flat sheaf. A morphism of regular filtered $\lambda$-flat sheaves $\left(\mathcal{E}_{1 *}, \mathbb{D}_{1}^{\lambda}\right) \longrightarrow\left(\mathcal{E}_{2 *}, \mathbb{D}_{2}^{\lambda}\right)$ is defined to be a morphism of the underlying $\mathcal{O}_{X}$-modules $\mathcal{E}_{1} \longrightarrow \mathcal{E}_{2}$ compatible with $\lambda$-connections and the filtrations.

If the underlying $\mathbf{c}$-parabolic sheaf $\mathcal{E}_{*}$ is a $\mathbf{c}$-parabolic bundle in codimension $k$, it is called a regular $\lambda$-flat $\mathbf{c}$-parabolic bundle in codimension $k$.

Remark 2.1 We do not recall the precise definition of $\mathbf{c}$-parabolic bundle. See Definition 3.12 of [14]. Although it is not difficult, it is a little complicated to state. It briefly means that $\mathcal{F}$ is a locally free $\mathcal{O}_{X}$-module, and that ${ }^{i} \mathrm{Gr}_{a}^{\mathcal{F}}(\mathcal{E})$ are locally free $\mathcal{O}_{D_{i}}$-modules. However, we need some compatibility conditions at the intersection of divisors in the case $\operatorname{dim} X \geq 3$. Note that it is equivalent to filtered bundle below, and J Iyer and C Simpson [9] and C Hertling and C Sevenheck [7] gave different but equivalent conditions.

Remark 2.2 We often omit to state "regular" in this paper, because we always assume regularity. The nonregular case is studied in [13].

Regular filtered $\lambda$-flat sheaf Let $\mathbf{E}_{*}=\left(\mathbf{E},\{\mathbf{c} E\} \mid \mathbf{c} \in \mathbf{R}^{S}\right)$ be a filtered sheaf on $(X, D)$. Namely:

- $\mathbf{E}$ is a torsion-free coherent $\mathcal{O}_{X}(* D)$-module.

- $\left\{{ }_{\mathbf{c}} E\right\}$ is an increasing filtration by coherent $\mathcal{O}_{X}$-submodules of $\mathbf{E}$ indexed by $\mathbf{R}^{S}$ such that (i) $\mathbf{E}_{\mid X-D}={ }_{\mathbf{c}} E_{\mid X-D}$ for any $\mathbf{c}$, (ii) ${ }_{\mathbf{a}} E=\bigcap_{\mathbf{a}<\mathbf{b}} \mathbf{b} E$, (iii) $\mathbf{E}=\bigcup_{\mathbf{a} \in \mathbf{R}^{S} \mathbf{a}} E$.

- $\mathbf{a}^{\prime} E={ }_{\mathbf{a}} E \otimes \mathcal{O}_{X}\left(-\sum n_{j} \cdot D_{j}\right)$ as submodules of $\mathbf{E}$, where $\mathbf{a}^{\prime}=\mathbf{a}-\left(n_{j} \mid j \in S\right)$. 
- For each $\mathbf{c} \in \mathbf{R}^{S}$, let ${ }^{i} \mathcal{F}$ be a filtration of ${ }_{\mathbf{c}} E$ indexed by $] c_{i}-1, c_{i}$ ] given as follows:

$$
{ }^{i} \mathcal{F}_{d}\left({ }_{\mathbf{c}} E\right):=\bigcup_{\substack{a_{i} \leq d \\ \mathbf{a} \leq \mathbf{c}}}{ }_{\mathbf{a}} E .
$$

Then the tuple ${ }_{\mathbf{c}} E_{*}:=\left({ }_{\mathbf{c}} E,\left\{{ }^{i} \mathcal{F} \mid i \in S\right\}\right)$ is a $\mathbf{c}$-parabolic sheaf, ie, the sets $\left\{a \mid{ }^{i} \mathrm{Gr}_{a}^{\mathcal{F}}(\mathbf{c} E) \neq 0\right\}$ are finite.

See Section 3.2 of [14] for more details on filtered sheaf. Each ${ }_{\mathbf{c}} E_{*}$ is called the $\mathbf{c}$-truncation of $\mathbf{E}_{*}$. We can reconstruct $\mathbf{E}_{*}$ from ${ }_{\mathbf{c}} E_{*}$. It is easy to observe that the notions of $\mathbf{c}$-parabolic sheaves and filtered sheaves are essentially equivalent. If each $\mathbf{c}$-truncation is a $\mathbf{c}$-parabolic bundle, $\mathbf{E}_{*}$ is called a filtered bundle. If there exists a closed subset $Z \subset X$ such that (i) $\operatorname{codim}_{X}(Z) \geq k$, (ii) $\mathbf{E}_{* \mid X-Z}$ is a filtered bundle, then $\mathbf{E}_{*}$ is called a filtered bundle in codimension $k$.

A regular flat $\lambda$-connection of $\mathbf{E}_{*}$ is defined to be a flat $\lambda$-connection $\mathbb{D}^{\lambda}$ of $\mathbf{E}$ satisfying $\mathbb{D}^{\lambda}\left({ }_{\mathbf{c}} e\right) \subset_{\mathbf{c}} E \otimes \Omega_{X}^{1,0}(\log D)$. Such a tuple $\left(\mathbf{E}_{*}, \mathbb{D}^{\lambda}\right)$ is called a regular filtered $\lambda$-flat sheaf. A morphism of regular filtered $\lambda$-flat sheaves $\left(\mathbf{E}_{1 *}, \mathbb{D}_{1}^{\lambda}\right) \longrightarrow\left(\mathbf{E}_{2 *}, \mathbb{D}_{2}^{\lambda}\right)$ is defined to be a morphism of the underlying $\mathcal{O}_{X}(* D)$-modules compatible with the $\lambda$-connections and the filtrations. It is easy to observe that the categories of regular filtered $\lambda$-flat sheaves and regular $\mathbf{c}$-parabolic sheaves are equivalent, given by the functor taking $\mathbf{c}$-truncations. We will not have to distinguish them so carefully.

If the underlying filtered sheaf is a filtered bundle in codimension $k$, it is called a regular filtered $\lambda$-flat bundle in codimension $k$.

Lemma 2.3 A regular filtered $\lambda$-flat sheaf on $(X, D)$ is a regular filtered $\lambda$-flat bundle in codimension one.

Proof We have only to check that there exists a subset $W \subset D$ with $\operatorname{codim}_{X}(W) \geq 2$, such that $E_{* \mid X \backslash W}$ is a c-parabolic bundle on $(X \backslash W, D \backslash W)$ for some c. We can take $W$ as $\bigcup_{i \neq j} D_{i} \cap D_{j} \subset W$, and hence we may assume $D$ is smooth. Since $E=\mathbf{E}_{\mid X-D}$ is locally free and $\mathbf{c} E$ is torsion-free, we can take $W^{\prime} \subset D$ with $\operatorname{codim}_{X}\left(W^{\prime}\right) \geq 2$ such that ${ }_{\mathrm{c}} E_{\mid X-W^{\prime}}$ is locally free. We may also take a subset $W^{\prime \prime} \subset D \backslash W^{\prime}$ with $\operatorname{codim}_{X}\left(W^{\prime \prime}\right) \geq 2$ such that the parabolic filtration of ${ }_{\mathbf{c}} E_{\mid D \backslash\left(W^{\prime} \cup W^{\prime \prime}\right)}$ is filtration in the category of vector bundles. Then $W=W^{\prime} \cup W^{\prime \prime}$ gives the desired subset.

Stability If $X$ is an $n$-dimensional projective variety with an ample line bundle $L$, we can define the $\mu$-stability, $\mu$-semistability, and $\mu$-polystability of regular filtered $\lambda$-flat sheaves with respect to $L$, in the standard manner. " $\mu$-stability with respect to $L "$ will be called $\mu_{L}$-stability, in this paper. 
Remarks about the terminology and the notation We give some remarks about the terminology "parabolic structure". We often study a regular c-parabolic $\lambda$-flat bundle on $(X, D)$ for some $\mathbf{c} \in \mathbf{R}^{S}$. In our most arguments, a choice of $\mathbf{c}$ are not relevant. In fact, $\mathbf{c}$ is fixed to be $(0, \ldots, 0)$ in many references where the parabolic structure is investigated. However, it is sometimes convenient to avoid the case $c_{i} \in \mathcal{P} a r\left({ }_{\mathbf{c}} E_{*}, i\right)$, for example, when we consider a perturbation of the parabolic structure. That is the main reason why we consider general $\mathbf{c}$-parabolic structure.

In the following argument, we often assume $c_{i} \notin \mathcal{P} \operatorname{ar}\left({ }_{\mathrm{c}} E_{*}, i\right)$ implicitly, and we often omit to distinguish c, and use the terminology "parabolic structure" instead of "c-parabolic structure", when we do not have to care about the choice of $\mathbf{c}$. The author hopes that there will be no risk of confusion and that it will reduce unnecessary complexity of the description.

Relatedly we have the remark about the notation for parabolic bundles. We often use the symbols $(\mathbf{c} E, \mathbf{F})$ or ${ }_{\mathbf{c}} E_{*}$ to denote a $\mathbf{c}$-parabolic bundle, when we would like to distinguish c. The symbol " $E$ " is also appropriate and useful, when we regard it as a prolongment of a locally free sheaf $E$ on $X-D$. But, in some case, a vector bundle is given not only on $X-D$ but also on $X$ from the beginning. And, as is said above, we will not care about the choice of $\mathbf{c}$. In such a case, we often prefer the symbols $(E, \mathbf{F})$ or $E_{*}$ for simplicity of the description.

One more remark is that we will not distinguish regular $\mathbf{c}$-parabolic sheaves and regular filtered $\lambda$-flat sheaves, because the notions are essentially equivalent.

2.1.2 KMS-structure, graded semisimplicity and SPW-condition We prepare some notation and conditions. Let $\left(\mathbf{E}_{*}, \mathbb{D}^{\lambda}\right)$ be a regular filtered $\lambda$-flat bundle over $(X, D)$. For simplicity, we consider only the case $\lambda \neq 0$. Let us take any element $\mathbf{c} \in \mathbf{R}^{S}$, and the $\mathbf{c}$-truncation ${ }_{\mathbf{c}} E_{*}$ of $\mathbf{E}_{*}$. Let $D_{I}:=\bigcap_{i \in I} D_{i}$ for any subset $I \subset S$. We have the induced filtrations ${ }^{i} F(i \in I)$ on ${ }_{\mathbf{c}} E_{\mid D_{I}}$. For $\left.\left.\mathbf{a} \in \prod_{i \in I}\right] c_{i}-1, c_{i}\right]$, we put

$$
{ }^{I} \mathrm{Gr}_{\mathbf{a}}^{F}(\mathbf{c} E):=\frac{{ }^{I} F_{\mathbf{a}}\left(\mathbf{c} E_{\mid D_{I}}\right)}{\sum_{\mathbf{b} \Varangle \mathbf{a}} I^{I} F_{\mathbf{b}}\left(\mathbf{c} E_{\mid D_{I}}\right)}, \quad{ }^{I} F_{\mathbf{a}}\left({ }_{\mathbf{c}} E_{\mid D_{I}}\right)=\bigcap_{i \in I}{ }^{i} F_{a_{i}}\left({ }_{\mathbf{c}} E_{\mid D_{I}}\right) .
$$

We obtain the following sets:

$$
\mathcal{P} a r\left(\mathbf{c} E_{*}, I\right):=\left\{\left.\mathbf{a}\right|^{I} \operatorname{Gr}_{\mathbf{a}}^{F}\left({ }_{\mathbf{c}} E\right) \neq 0\right\}, \quad \mathcal{P} a r\left(\mathbf{E}_{*}, I\right):=\bigcup_{\mathbf{c} \in \mathbf{R}^{S}} \mathcal{P} a r\left({ }_{\mathbf{c}} E_{*}, I\right)
$$

Any elements of these sets are called parabolic weights. Due to the regularity, we have the residue endomorphism $\operatorname{Res}_{i}\left(\mathbb{D}^{\lambda}\right)(i \in I)$ on $E_{\mathbf{c}} E_{\mid D_{I}}$, which preserves the filtrations ${ }^{j} F(j \in I)$. (Such a tuple of filtrations and endomorphisms is called KMS-structure.) 
Hence we have the induced endomorphism ${ }^{I} \mathrm{Gr}_{\mathbf{a}}^{F} \operatorname{Res}_{i}\left(\mathbb{D}^{\lambda}\right)$ of ${ }^{I} \mathrm{Gr}_{\mathbf{a}}^{F}\left({ }_{\mathbf{c}} e\right)$. We remark that the eigenvalues of $\operatorname{Res}_{i}\left(\mathbb{D}^{\lambda}\right)$ are constant on $D_{i}$. In particular, we obtain the generalized eigen decomposition:

$$
{ }^{I} \mathrm{Gr}_{\mathbf{a}}^{F}(\mathbf{c} E)=\bigoplus_{\boldsymbol{\alpha} \in \mathbf{C}^{I}}{ }^{I} \mathrm{Gr}_{\mathbf{a}, \boldsymbol{\alpha}}^{F, \mathbb{E}}(\mathbf{c} E) .
$$

We put $\left.\left.\mathcal{K} \mathcal{M S}\left({ }_{\mathbf{c}} E_{*}, I\right):=\left\{(\mathbf{a}, \boldsymbol{\alpha}) \in \prod_{i \in I}(] c_{i}-1, c_{i}\right] \times \mathbf{C}\right) \mid{ }^{I} \mathrm{Gr}_{\mathbf{a}, \boldsymbol{\alpha}}^{F, \mathbb{E}}\left({ }_{\mathbf{c}} E_{\mid D_{I}}\right) \neq 0\right\}$. The sets

$$
\mathcal{K M S}\left({ }_{\mathbf{c}} E_{*}, I\right), \quad \mathcal{K} \mathcal{M S}\left(\mathbf{E}_{*}, I\right):=\bigcup_{\mathbf{c} \in \mathbf{R}^{S}} \mathcal{K} \mathcal{M S}\left({ }_{\mathbf{c}} E_{*}, I\right)
$$

are called the KMS-spectrum.

Remark 2.4 In our other papers [15; 14; 13], an element of the above sets (2) is called a KMS-spectrum. We follow the suggestion of the referee in this paper.

Remark 2.5 Although we assumed that $\left(\mathbf{E}_{*}, \mathbb{D}^{\lambda}\right)$ is a regular filtered $\lambda$-flat bundle, the above sets make sense for $I \subset S$ with $|I| \leq k$ if $\left(\mathbf{E}_{*}, \mathbb{D}^{\lambda}\right)$ is a regular filtered $\lambda$-flat bundle in codimension $k$. In particular, we always have the sets $\operatorname{Par}\left(\mathbf{E}_{*}, i\right)$, $\mathcal{K} \mathcal{M S}\left(\mathbf{E}_{*}, i\right)$, etc., for $i \in S$.

Definition 2.6 We introduce two auxiliary conditions on $\left(\mathbf{E}_{*}, \mathbb{D}^{\lambda}\right)$.

- (Graded semisimple) The nilpotent parts of $\operatorname{Gr}_{a}^{F} \operatorname{Res}_{i}\left(\mathbb{D}^{\lambda}\right) \in \operatorname{End}\left({ }^{i} \operatorname{Gr}_{a}^{F}\left({ }_{c} E\right)\right)$ are 0 for any $i \in S, \mathbf{c} \in \mathbf{R}^{S}$ and $a \in \mathbf{R}$.

- $(S P W)$ There exist a positive integer $m$ and real numbers $\gamma_{i}(i \in S)$ such that $\mathcal{P a r}\left(\mathbf{E}_{*}, i\right)$ is contained in $\left\{\gamma_{i}+p / m \mid p \in \mathbf{Z}\right\}$ for each $i$.

We will study $\left({ }_{\mathbf{c}} E_{*}, \mathbb{D}^{\lambda}\right)$ satisfying these conditions in Section 3 as a preparation for our main theorem.

2.1.3 Saturated regular filtered $\lambda$-flat sheaf We introduce a nice class of regular filtered $\lambda$-flat sheaves. Let $\left(\mathbf{E}_{*}, \mathbb{D}^{\lambda}\right)$ be a regular filtered $\lambda$-flat sheaf on $(X, D)$.

Definition 2.7 $\left(\mathbf{E}_{*}, \mathbb{D}^{\lambda}\right)$ is called saturated, if there exists a subset $Z \subset D$ with $\operatorname{codim}_{X}(Z) \geq 2$ such that each $\mathbf{a}_{\mathbf{a}} E$ are determined on ${ }_{\mathbf{a}} E_{\mid X-Z}$. Namely, for any open subset $U \subset X$, the following holds:

$$
{ }_{\mathbf{a}} E(U)={ }_{\mathbf{a}} E(U \backslash Z) \cap \mathbf{E}(U)
$$

It is easy to see that a regular filtered $\lambda$-flat bundle is saturated. 
Lemma 2.8 If $\left(\mathbf{E}_{*}, \mathbb{D}^{\lambda}\right)$ is saturated, each $\mathbf{c}$-truncation ${ }_{\mathbf{c}} E$ is reflexive.

Proof Recall we have already known that $E_{*}$ is a filtered bundle in codimension one (Lemma 2.3). Let $E_{\mathbf{c}} E^{\vee \vee}$ denote the double dual of $\mathbf{c} E$. We have the naturally defined injective map ${ }_{\mathrm{c}} E \longrightarrow{ }_{\mathrm{c}} E^{\vee \vee}$. Due to the saturatedness, any sections of ${ }_{\mathrm{c}} E^{\vee \vee}$ naturally gives sections of ${ }_{\mathrm{c}} E$, ie, ${ }_{\mathrm{c}} E$ is isomorphic to ${ }_{\mathrm{c}} E^{\vee \vee}$.

Lemma 2.9 A saturated regular filtered $\lambda$-flat sheaf $\left(\mathbf{E}_{*}, \mathbb{D}^{\lambda}\right)$ on $(X, D)$ is a regular filtered $\lambda$-flat bundle in codimension two.

Proof We have only to show that there exists a subset $Z \subset D$ with $\operatorname{codim}_{X}(Z) \geq 3$ such that $E_{* \mid X-Z}$ is a $\mathbf{c}$-parabolic bundle on $(X-Z, D-Z)$ for any c. Because ${ }_{\mathbf{c}+\mathbf{b}} E={ }_{\mathbf{c}} E \otimes \mathcal{O}(\mathbf{b} \cdot D)$, where $\mathbf{b} \cdot D=\sum_{i \in S} b_{i} \cdot D_{i}$, we have only to show such a claim for a finite number of tuples c. Due to Lemma 2.8, there exists a subset $Z^{\prime} \subset D$ with $\operatorname{codim}_{X}\left(Z^{\prime}\right) \geq 3$ such that ${ }_{\mathbf{c}} E_{\mid X-Z^{\prime}}$ is locally free. Hence, we can assume that ${ }_{\mathrm{c}} E$ is locally free from the beginning.

We have the parabolic filtration ${ }^{i} F=\left\{{ }^{i} F_{a} \mid c_{i}-1<a \leq c_{i}\right\}$ of ${ }_{\mathbf{c}} E_{\mid D_{i}}$. We can take the saturation ${ }^{i} \widetilde{F}_{a}$ of ${ }^{i} F_{a}$. Namely, we put $G_{a}:={ }_{\mathrm{c}} E_{\mid D_{i}} /{ }^{i} F_{a}$, and let $G_{a}$ tor denote the torsion-part of $G_{a}$. Let $\pi_{a}:{ }_{\mathrm{c}} E_{\mid D_{i}} \longrightarrow G_{a}$ denote the projection, and we put ${ }^{i} \widetilde{F}_{a}:=\pi_{a}^{-1}\left(G_{a \text { tor }}\right)$.

\section{Lemma 2.10 ${ }^{i} \widetilde{F}_{a}={ }^{i} F_{a}$.}

Proof By our construction, we have ${ }^{i} F_{a} \subset{ }^{i} \widetilde{F}_{a}$, and we also know that there exists a subset $W \subset D_{i}$ with $\operatorname{codim}_{D_{i}}(W) \geq 1$ such that ${ }^{i} F_{a \mid D_{i}-W}={ }^{i} \widetilde{F}_{a \mid D_{i}-W}$.

Let $P$ be any point of $D_{i}$. Let $g$ be a germ of a section of ${ }^{i} \widetilde{F}_{a}$ at $P$, and let $G$ be a local section of $\mathbf{c} E$ on an open neighbourhood $U$ of $P$ in $X$ such that the germ of the restriction of $G$ to $D_{i}$ gives $g$. Then $G_{\mid U \backslash W}$ gives a section of ${ }_{\mathbf{c}^{\prime}} E$ on $U \backslash W$, where $\mathbf{c}^{\prime}=\left(c_{j}^{\prime}\right)$ is determined by $c_{j}^{\prime}=c_{j}(j \neq i)$ and $c_{i}=a$. Due to the saturatedness, $G$ is a section of $\mathbf{c}^{\prime} E$ on $U$. Thus, $g$ is the germ of a section of ${ }^{i} F_{a}$, and ${ }^{i} F_{a}={ }^{i} \widetilde{F}_{a}$. Hence, we obtain Lemma 2.10 .

Let us return to the proof of Lemma 2.9. Due to Lemma 2.10, the associated graded vector bundle ${ }^{i} \mathrm{Gr}^{F}\left({ }_{\mathrm{c}} E_{\mid D_{i}}\right)$ is torsion free. Hence, there exists a subset $Z_{i}^{\prime \prime} \subset D_{i}$ with $\operatorname{codim}_{D_{i}} Z_{i}^{\prime \prime} \geq 2$ such that ${ }^{i} F_{\mid D_{i} \backslash Z_{i}^{\prime \prime}}$ is a filtration in the category of vector bundles on $D_{i}^{\prime \prime} \backslash Z_{i}^{\prime \prime}$. Then ${ }_{\mathbf{c}} E_{* \mid X-Z^{\prime \prime}}$ is a c-parabolic locally free sheaf on $\left(X-Z^{\prime \prime}, D-Z^{\prime \prime}\right)$. Thus we are done.

Remark 2.11 By the correspondence of saturated regular filtered flat sheaves and filtered local systems, we can obtain more concrete picture of the saturated regular filtered flat sheaves. See Section 6. 
2.1.4 Canonical decomposition Let $X$ be a smooth irreducible projective variety with an ample line bundle $L$. Let $D$ be a simple normal crossing hypersurface of $X$. Let $\left(\mathcal{E}_{*}^{(i)}, \mathbb{D}^{\lambda(i)}\right)(i=1,2)$ be $\mu_{L}$-semistable regular $\mathbf{c}$-parabolic $\lambda$-flat sheaves on $(X, D)$ such that $\mu_{L}\left(\mathcal{E}_{*}^{(1)}\right)=\mu_{L}\left(\mathcal{E}_{*}^{(2)}\right)$. Let $f:\left(\mathcal{E}_{*}^{(1)}, \mathbb{D}^{\lambda(1)}\right) \longrightarrow\left(\mathcal{E}_{*}^{(2)}, \mathbb{D}^{\lambda(2)}\right)$ be a nontrivial morphism. Let $\left(\mathcal{K}_{*}, \mathbb{D}_{\mathcal{K}}^{\lambda}\right)$ denote the kernel of $f$, which is naturally equipped with the parabolic structure and the flat $\lambda$-connection. Let $\mathcal{I}$ denote the image of $f$, and $\tilde{\mathcal{I}}$ denote the saturated subsheaf of $\mathcal{E}^{(2)}$ generated by $\mathcal{I}$. The parabolic structures of $\mathcal{E}_{*}^{(1)}$ and $\mathcal{E}_{*}^{(2)}$ induce the parabolic structures of $\mathcal{I}$ and $\tilde{\mathcal{I}}$, respectively. We denote the induced parabolic flat sheaves by $\left(\mathcal{I}_{*}, \mathbb{D}_{\mathcal{I}}^{\lambda}\right)$ and $\left(\widetilde{\mathcal{I}}_{*}, \mathbb{D}_{\widetilde{\mathcal{I}}}^{\lambda}\right)$. The following lemma can be shown by the same argument as the proof of Lemma 3.9 of [14].

Lemma $2.12\left(\mathcal{K}_{*}, \mathbb{D}_{\mathcal{K}}^{\lambda}\right),\left(\mathcal{I}_{*}, \mathbb{D}_{\mathcal{I}}^{\lambda}\right)$ and $\left(\widetilde{\mathcal{I}}_{*}, \mathbb{D}_{\widetilde{\mathcal{I}}}^{\lambda}\right)$ are also $\mu_{L}$-semistable such that $\mu_{L}\left(\mathcal{K}_{*}\right)=\mu_{L}\left(\mathcal{I}_{*}\right)=\mu_{L}\left(\widetilde{\mathcal{I}}_{*}\right)=\mu_{L}\left(\mathcal{E}_{*}^{(i)}\right)$. Moreover, $\mathcal{I}_{*}$ and $\tilde{\mathcal{I}}_{*}$ are isomorphic in codimension one.

Lemma 2.13 Let $\left(\mathcal{E}_{*}^{(i)}, \mathbb{D}^{\lambda(i)}\right)(i=1,2)$ be $\mu_{L}$-semistable reflexive saturated regular parabolic $\lambda$-flat sheaves such that $\mu_{L}\left(\mathcal{E}_{*}^{(1)}\right)=\mu_{L}\left(\mathcal{E}_{*}^{(2)}\right)$. Assume either one of the following:

(1) One of $\left(\mathcal{E}_{*}^{(i)}, \mathbb{D}^{\lambda(i)}\right)$ is $\mu_{L}$-stable, and $\operatorname{rank}\left(\mathcal{E}^{(1)}\right)=\operatorname{rank}\left(\mathcal{E}^{(2)}\right)$ holds.

(2) Both $\left(\mathcal{E}_{*}^{(i)}, \mathbb{D}^{\lambda(i)}\right)$ are $\mu_{L}-$ stable.

If there is a nontrivial morphism $f:\left(\mathcal{E}_{*}^{(1)}, \mathbb{D}^{\lambda(1)}\right) \longrightarrow\left(\mathcal{E}_{*}^{(2)}, \mathbb{D}^{\lambda(2)}\right)$, then $f$ is an isomorphism.

Proof If $\left(\mathcal{E}_{*}^{(1)}, \mathbb{D}^{\lambda(1)}\right)$ is $\mu_{L}$-stable, the kernel of $f$ is trivial due to Lemma 2.12. If $\left(\mathcal{E}_{*}^{(2)}, \mathbb{D}^{\lambda(2)}\right)$ is $\mu_{L}$-stable, the image of $f$ and $\mathcal{E}^{(2)}$ are the same at the generic point of $X$. Thus, we obtain that $f$ is generically isomorphic in any case. Then we obtain that $f$ is isomorphic in codimension one, due to Lemma 3.7 of [14]. Since both $\mathcal{E}_{*}^{(i)}$ are reflexive and saturated, we obtain that $f$ is isomorphic.

Corollary 2.14 Let $\left(\mathcal{E}_{*}, \mathbb{D}^{\lambda}\right)$ be a $\mu_{L}$-polystable reflexive saturated regular parabolic $\lambda$-flat sheaf. Then we have the unique decomposition:

$$
\left(\mathcal{E}_{*}, \mathbb{D}^{\lambda}\right)=\bigoplus_{j}\left(\mathcal{E}_{*}^{(j)}, \mathbb{D}^{\lambda(j)}\right) \otimes \mathbf{C}^{m(j)}
$$

Here, $\left(\mathcal{E}_{*}^{(j)}, \mathbb{D}^{\lambda(j)}\right)$ are $\mu_{L}$-stable with $\mu_{L}\left(\mathcal{E}_{*}^{(j)}\right)=\mu\left(\mathcal{E}_{*}\right)$, and they are mutually nonisomorphic. It is called the canonical decomposition in the rest of the paper. 


\subsubsection{Prolongment of flat subbundle and a Mehta-Ramanathan type theorem} Mehta-Ramanathan type theorems are one of the most important and standard tools in the study of stable objects on projective varieties (Proposition 2.21). In the case of regular filtered $\lambda$-flat sheaves, it can be proved in a much more elementary way.

To begin with, we recall a well known fact about regular singularity of a flat meromorphic connection.

Lemma 2.15 Let $E$ be a holomorphic bundle on a disc $\Delta$, and let $\nabla$ be a logarithmic flat connection of $E$ on $(\Delta, O)$, ie, $\nabla(E) \subset E \otimes \Omega_{\Delta}^{1,0}(\log O)$. Let $f$ be a flat section of $E_{\mid \Delta^{*}}$. Then $f$ naturally gives a meromorphic section of $E$.

Corollary 2.16 We put $X=\Delta_{z} \times \Delta_{w}^{n}$ and $D=\{0\} \times \Delta_{w}^{n}$. Let $E$ be a holomorphic vector bundle on $X$ and $\nabla$ be the logarithmic flat connection of $E$ on $(X, D)$. Let $e$ be a flat section of $E_{\mid X-D}$.

- $e$ gives a meromorphic section of $E$.

- Assume that $e$ is holomorphic on $E$ and that $e_{\mid Q} \neq 0$ for some $Q \in D$. Then $e_{\mid Q^{\prime}} \neq 0$ for any $Q^{\prime} \in D$.

Proof We may assume that we have a holomorphic frame $\mathbf{v}$ of $E$. We have the expression $e=\sum f_{i}(z, w) \cdot v_{i}$. When we fix $w$, then $f_{i}(z, w)$ are meromorphic with respect to $z$. Thus, we have the least integer $j(w)$ such that the orders of the poles of $f_{i}(z, w)$ are less than $j(w)$. We put $\mathcal{S}_{j}:=\{w \mid j(w) \leq j\}$. We have $D=\bigcup_{j} \mathcal{S}_{j}$. If $\mathcal{S}_{j} \neq D$, the measure of $\mathcal{S}_{j}$ is 0 . Hence, we obtain $\mathcal{S}_{j}=D$ for some $j$, which means $e$ is meromorphic. Thus, we obtain the first claim.

Assume that $e$ is holomorphic and that $e_{\mid Q} \neq 0$ for some $Q \in D$. Recall that we have the induced connection ${ }^{D} \nabla$ of $E_{\mid D}$. Namely, for any holomorphic section $f \in E_{\mid D}$, take a holomorphic $F \in E$ such that $F_{\mid D}=f$, and then ${ }^{D} \nabla(f):=\nabla(F)_{\mid D}$ is well defined. Because ${ }^{D} \nabla\left(e_{\mid D}\right)=0$, we obtain the second claim.

Corollary 2.17 We put $X=\Delta^{n}, D_{i}=\left\{z_{i}=0\right\}$ and $D=\bigcup_{i=1}^{n} D_{i}$. Let $(E, \nabla)$ be a logarithmic connection on $(X, D)$, and let $e$ be a flat section on $X-D$.

- $e$ gives a meromorphic section of $E$.

- Assume that $e$ is holomorphic. We put $D_{i}^{\circ}:=D_{i} \backslash \bigcup_{j \neq i} D_{j}$. If $e_{\mid Q} \neq 0$ for some $Q \in D_{i}^{\circ}$, we have $e_{\mid Q^{\prime}} \neq 0$ for any $Q^{\prime} \in D_{i}^{\circ}$. 
Let $X$ be a complex manifold, and let $D$ be a normal crossing divisor of $X$. Let $(E, \nabla)$ be a flat bundle on $X-D$. Recall that P Deligne gave the extension $\widetilde{E}$ of $E$ in [3], such that (i) $\widetilde{E}_{\mid X-D}=E$, (ii) $\nabla(\widetilde{E}) \subset \widetilde{E} \otimes \Omega^{1,0}(\log D)$, (iii) the real parts of the eigenvalues of $\operatorname{Res}_{i}(\nabla)$ are contained in $\{0 \leq t<1\}$. Such an extension is unique, or in other words, it is unique as the subsheaf of $\iota_{*} E$, where $\iota$ denotes the inclusion $X-D \longrightarrow X$. If $\lambda \neq 0$, the prolongment can also be done for $\lambda$-flat bundle $\left(E, \mathbb{D}^{\lambda}\right)$ on $X-D$, or more precisely, for the associated flat bundle $\left(E, \mathbb{D}^{\lambda f}\right)$.

Lemma 2.18 Let $\left(\mathbf{E}_{*}, \mathbb{D}^{\lambda}\right)$ be a regular filtered $\lambda$-flat bundle on $(X, D)$, and we put $\left(E, \mathbb{D}^{\lambda}\right):=\left(\mathbf{E}_{*}, \mathbb{D}^{\lambda}\right)_{\mid X-D}$. Let $\left(\widetilde{E}, \mathbb{D}^{\lambda}\right)$ be the Deligne extension of $\left(E, \mathbb{D}^{\lambda}\right)$. Then we have $\mathbf{E}=\widetilde{E} \otimes \mathcal{O}_{X}(* D)$, where $\mathcal{O}_{X}(* D)$ denotes the sheaf of meromorphic functions on $X$ whose poles are contained in $D$.

Proof We have the naturally defined flat section $s$ on $\operatorname{Hom}(\mathbf{c} E, \widetilde{E})_{\mid X-D}$. Due to Corollary 2.17, $s$ is a meromorphic section, and hence we obtain the flat inclusion c $E \longrightarrow \widetilde{E} \otimes \mathcal{O}(N \cdot D)$ for some large integer $N$, which induce the morphism $\mathbf{E}=$ $\bigcup_{\mathbf{c}} E={ }_{\mathbf{c}} E \otimes \mathcal{O}(* D) \longrightarrow \widetilde{E} \otimes \mathcal{O}(* D)$. Similarly, we obtain the inclusion $\widetilde{E} \longrightarrow$ c $E \otimes \mathcal{O}(N \cdot D)$, and $\widetilde{E} \otimes \mathcal{O}(* D) \longrightarrow \mathbf{E}$. They are clearly mutually inverse.

Lemma 2.19 Let $\left(\mathbf{E}_{*}, \mathbb{D}^{\lambda}\right)$ be a regular filtered $\lambda$-flat sheaf on $(X, D)$, and let $\left(\widetilde{E}, \mathbb{D}^{\lambda}\right)$ be as in the previous lemma. Then we have $\mathbf{E} \simeq \widetilde{E} \otimes \mathcal{O}(* D)$ naturally.

Proof Due to Lemma 2.3 and Lemma 2.18, there exists a subset $W \subset D$ with $\operatorname{codim}_{X}(W) \geq 2$ such that $\mathbf{E}_{\mid X-W} \simeq \widetilde{E} \otimes \mathcal{O}(* D)_{\mid X-W}$. Let us fix c. There exists a large integer $N$ such that we have ${ }_{\mathbf{c}} E_{\mid X-W} \subset \widetilde{E} \otimes \mathcal{O}(N \cdot D)_{\mid X-W}$. Since $\widetilde{E}$ is locally free, we obtain ${ }_{\mathbf{c}} E \subset \widetilde{E} \otimes \mathcal{O}(N \cdot D)$, and thus $\mathbf{E} \subset \widetilde{E} \otimes \mathcal{O}(* D)$. On the other hand, there exists a large integer $N^{\prime}$ such that $\widetilde{E}_{\mid X-W} \subset \mathbf{c} E \otimes \mathcal{O}\left(N^{\prime} \cdot D\right)_{\mid X-W}$. Hence, $\widetilde{E} \subset{ }_{\mathbf{c}} E^{\vee \vee} \otimes \mathcal{O}\left(N^{\prime} \cdot D\right)$, where ${ }_{\mathbf{c}} E^{\vee \vee}$ denotes the double dual of $\mathbf{c}_{\mathbf{c}} E$. Hence, we obtain $\widetilde{E} \otimes \mathcal{O}(* D) \subset_{\mathbf{c}} E^{\vee \vee} \otimes \mathcal{O}(* D)$. It is easy to see ${ }_{\mathrm{c}} E^{\vee \vee} \otimes \mathcal{O}(* D) \simeq{ }_{\mathrm{c}} E \otimes \mathcal{O}(* D)$. Thus we are done.

Lemma 2.20 Let $\left(\mathbf{E}_{*}, \mathbb{D}^{\lambda}\right)$ be a regular filtered $\lambda$-flat sheaf on $(X, D)$, and we put $\left(E, \mathbb{D}^{\lambda}\right):=\left(\mathbf{E}_{*}, \mathbb{D}^{\lambda}\right)_{\mid X-D}$. Let $E^{\prime}$ be a $\lambda$-flat subbundle of $E$. Then we have the corresponding regular filtered $\lambda$-flat subsheaf $\mathbf{E}_{*}^{\prime} \subset \mathbf{E}_{*}$ such that $E_{\mathbf{c}}^{\prime}$ are saturated in ${ }_{\mathrm{c}} E, \mathrm{ie}, \mathrm{c}_{\mathrm{c}} E / \mathrm{c}_{\mathrm{c}} E^{\prime}$ is torsion-free.

Proof Let $\widetilde{E}$ denote the Deligne extension of $\left(E, \mathbb{D}^{\lambda}\right)$. We have the corresponding subbundle $\widetilde{E}^{\prime} \subset \widetilde{E}$. Therefore, we obtain $\widetilde{\mathbf{E}}^{\prime}:=\widetilde{E}^{\prime} \otimes \mathcal{O}(* D) \subset \widetilde{E} \otimes \mathcal{O}(* D)=\mathbf{E}$. For each $\mathbf{c}$, the c-truncation ${ }_{\mathbf{c}} E^{\prime}$ is given by the intersection of ${ }_{\mathbf{c}} E$ and $\mathbf{E}^{\prime}$ in $\mathbf{E}$. Or equivalently, $E^{\prime}$ can be given by the intersection of ${ }_{\mathbf{c}} E$ and $\widetilde{E^{\prime}}(N \cdot D)$ in $\widetilde{E}(N \cdot D)$ for a sufficiently large $N$. Thus, we obtain $\mathbf{E}_{*}^{\prime} \subset \mathbf{E}_{*}$. 
Let us show the Mehta-Ramanathan type theorem for regular filtered $\lambda$-flat sheaves. Let $X$ be a smooth irreducible projective variety with an ample line bundle $L$ and a simple normal crossing divisor $D$. Let $\left(\mathbf{E}_{*}, \mathbb{D}^{\lambda}\right)$ be a regular filtered $\lambda$-flat sheaf on $(X, D)$. Let $N$ be a sufficiently large number. We can take a generic hyper-plane section $Y$ of $L^{\otimes N}$ satisfying the properties: (i) $D_{Y}:=Y \cap D$ is simply normal crossing in $Y$, (ii) $\pi_{1}(Y \backslash D) \longrightarrow \pi_{1}(X \backslash D)$ is surjective.

Proposition 2.21 Assume $\operatorname{dim} X \geq 2$. Then $\left(\mathbf{E}_{*}, \mathbb{D}^{\lambda}\right)$ is $\mu_{L}-$ stable, if and only if $\left(\mathbf{E}_{*}, \mathbb{D}^{\lambda}\right)_{\mid Y}$ is $\mu_{L}-$ stable.

Proof Let us fix c. If $W \subset_{\mathbf{c}} E$ destabilizes, the restriction $W_{\mid Y}$ clearly destabilizes. Hence, the $\mu_{L}$-stability of $\left({ }_{\mathbf{c}} E_{*}, \mathbb{D}^{\lambda}\right)_{\mid Y}$ implies the $\mu_{L}$-stability of $\left({ }_{\mathbf{c}} E_{*}, \mathbb{D}^{\lambda}\right)$. Assume $\left({ }_{\mathbf{c}} E_{*}, \mathbb{D}^{\lambda}\right)_{\mid Y}$ is not $\mu_{L}$-stable, and let $W$ be a subsheaf of ${ }_{\mathbf{c}} E_{\mid Y}$ satisfying $\mathbb{D}^{\lambda}(W) \subset W \otimes \Omega_{Y}^{1,0}\left(\log D_{Y}\right)$ and $\operatorname{par}-\operatorname{deg}\left(W_{*}\right) / \operatorname{rank}(W) \geq \operatorname{par}-\operatorname{deg}\left({ }_{\mathbf{c}} E_{*}\right) / \operatorname{rank} E$. Let $Q$ be any point of $X-D$. Take a path $\gamma$ connecting $Q$ and a point $P$ of $Y \backslash D$. By the parallel transport along the path, we obtain the vector subspace $W_{Q}^{\prime} \subset E_{\mid Q}$. It is independent of choices of $P$ and $\gamma$, and we obtain the flat subbundle $W^{\prime} \subset_{\mathbf{c}} E_{\mid X-D}$. Due to Lemma 2.20, we obtain the saturated subsheaf $\widetilde{W}^{\prime} \subset{ }_{\mathbf{c}} E$. By a general argument, it can be shown that there exists a subset $Z \subset D$ with $\operatorname{codim}_{X}(Z) \geq 2$ such that $\widetilde{W}_{* \mid X-Z}^{\prime}$ is a parabolic subbundle of $\mathbf{c} E_{\mid X-Z}$. Then it is easy to check that $\widetilde{W}^{\prime}$ destabilizes.

2.1.6 Perturbation of parabolic structure We recall the method of perturbation of parabolic structure, which is one of the main ideas in [14] and this paper.

Let $X$ be a smooth projective surface with an ample line bundle $L$, and $D$ be a simple normal crossing hypersurface with the irreducible decomposition $D=\bigcup_{i \in S} D_{i}$. Note that each $D_{i}$ is smooth by the assumption. Let $\left({ }_{\mathbf{c}} E, \mathbf{F}, \mathbb{D}^{\lambda}\right)$ be a regular $\mathbf{c}$-parabolic $\lambda$-flat bundle over $(X, D)$ for some $\mathbf{c} \in \mathbf{R}^{S}$. Assume $\lambda \neq 0$. We also assume $c_{i} \notin \mathcal{P a r}\left({ }_{\mathbf{c}} E, \mathbf{F}, i\right)$ for each $i \in S$, for simplicity. Let $\mathcal{N}_{i}$ denote the nilpotent part of the induced endomorphism $\mathrm{Gr}^{F} \operatorname{Res}_{i}\left(\mathbb{D}^{\lambda}\right)$ on ${ }^{i} \mathrm{Gr}_{a}^{F}(\mathrm{c} E)$. We would like to consider perturbation of parabolic structure, as in Section 3.4 of [14]. First, we will recall a general construction. Then we will give two kinds of more specified perturbations.

Let $\eta$ be a generic point of $D_{i}$. We have the weight filtration $W_{\eta}$ of the nilpotent map $\mathcal{N}_{i, \eta}$ on ${ }^{i} \mathrm{Gr}^{F}\left({ }_{\mathbf{c}} E\right)_{\eta}$, which is indexed by $\mathbf{Z}$. Then we can extend it to the filtration $W$ of ${ }^{i} \mathrm{Gr}^{F}(\mathrm{c} E)$ in the category of vector bundles on $D_{i}$ because $D_{i}$ are smooth curves. By our construction, $\mathcal{N}_{i}\left(W_{k}\right) \subset W_{k-2}$. The endomorphism $\operatorname{Res}_{i}\left(\mathbb{D}^{\lambda}\right)$ preserves the filtration $W$ on ${ }^{i} \mathrm{Gr}^{F}\left({ }_{\mathbf{c}} E\right)$, and the nilpotent part of the induced endomorphisms on $\mathrm{Gr}^{W}\left({ }^{i} \mathrm{Gr}^{F}\left({ }_{\mathrm{c}} E\right)\right)$ are trivial. Recall that the flat $\lambda$-connection $\mathbb{D}^{\lambda}$ locally induces the 
$\lambda$-connection ${ }^{i} \mathbb{D}^{\lambda}$ of the vector bundle ${ }_{\mathrm{c}} E_{\mid D_{i}}$ on $D_{i}$. Since ${ }^{i} \mathrm{Gr}^{F}\left({ }^{i} \mathbb{D}^{\lambda}\right)$ commutes with $\operatorname{Res}_{i} \mathbb{D}^{\lambda}$, it preserves the filtration $W$.

Let us take a refinement of the filtration ${ }^{i} F$ for each $i$. For any $\left.\left.a \in\right] c_{i}-1, c_{i}\right]$, we have the surjection $\pi_{a}:{ }^{i} F_{a}\left({ }_{\mathrm{c}} E_{\mid D_{i}}\right) \longrightarrow{ }^{i} \mathrm{Gr}_{a}{ }_{a}\left({ }_{\mathrm{c}} E\right)$. We put ${ }^{i} \widetilde{F}_{a, k}:=\pi_{a}^{-1}\left(W_{k}\right)$. Thus, we obtain the increasing filtration ${ }^{i} \widetilde{F}$ indexed by $\left.] c_{i}-1, c_{i}\right] \times \underset{\widetilde{Z}}{\mathbf{Z}}$ with the lexicographic order. Obviously, the set $\left.\left.\widetilde{S}_{i}:=\{(a, k) \in] c_{i}-1, c_{i}\right] \times \mathbf{Z} \mid{ }^{i} \mathrm{Gr}_{(a, k)} \neq 0\right\}$ is finite.

Let us explain an $\epsilon$-perturbation. Let $\left.\varphi_{i}: \widetilde{S}_{i} \longrightarrow\right] c_{i}-1, c_{i}$ ] be increasing maps such that $\left|\varphi_{i}(a, k)-a\right| \leq C \cdot \epsilon$ for some $C>0$. (Since we are interested in the family of filtrations $\mathbf{F}^{(\epsilon)}(\epsilon>0)$, this condition makes sense.) Then ${ }^{i} \widetilde{F}$ and $\varphi_{i}$ give a $\mathbf{c}$-parabolic filtration $\mathbf{F}^{(\epsilon)}=\left({ }^{i} F^{(\epsilon)} \mid i \in S\right)$, and we obtain a regular $\mathbf{c}$-parabolic $\lambda$-flat bundle $\left({ }_{\mathbf{c}} E, \mathbf{F}^{(\epsilon)}, \mathbb{D}^{\lambda}\right)$ called an $\epsilon$-perturbation of $\left({ }_{\mathbf{c}} E, \mathbf{F}, \mathbb{D}^{\lambda}\right)$. We mention two properties which are clear from the construction.

- We have the following convergence in the cohomology group $H^{*}(X, \mathbf{R})$.

$$
\begin{aligned}
& \lim _{\epsilon \rightarrow 0} \operatorname{par} \mathrm{c}_{1}\left(\mathbf{c} E, \mathbf{F}^{(\epsilon)}\right)=\operatorname{par}^{-c_{1}}(\mathbf{c} E, \mathbf{F}), \\
& \lim _{\epsilon \rightarrow 0} \operatorname{par}^{-c_{2}}\left(\mathbf{c} E, \mathbf{F}^{(\epsilon)}\right)=\operatorname{par}_{-c_{2}}(\mathbf{c} E, \mathbf{F})
\end{aligned}
$$

(See Sections 3.1.2 and 3.1.5 of [14] for $\operatorname{par}^{-c_{1}}\left({ }_{\mathbf{c}} E, \mathbf{F}\right)$ and $\left.\operatorname{par}^{-c_{2}}\left({ }_{\mathbf{c}} E, \mathbf{F}\right).\right)$

- $\left({ }_{\mathbf{c}} E, \mathbf{F}^{(\epsilon)}, \mathbb{D}^{\lambda}\right)$ is graded semisimple (Definition 2.6).

The following proposition is standard. (See Proposition 3.28 of [14], for example.)

Proposition 2.22 Assume that $\left({ }_{\mathbf{c}} E, \mathbf{F}, \mathbb{D}^{\lambda}\right)$ is $\mu_{L}$-stable. If $\epsilon$ is sufficiently small, the $\epsilon$-perturbation $\left(\mathbf{c} E, \mathbf{F}^{(\epsilon)}, \mathbb{D}^{\lambda}\right)$ is also $\mu_{L}$-stable.

We will use two kinds of perturbations $\varphi_{i}$ of weights.

(I) The image of $\varphi_{i}$ is contained in $\mathbf{Q}$ for each $i \in S$. This kind of perturbation will be used to obtain a formula to express the parabolic characteristic numbers in terms of the boundary data (Section 3.5).

(II) For simplicity, we assume $\epsilon=m^{-1}$ and $0<10 \operatorname{rank} E \cdot \epsilon<\operatorname{gap}\left({ }_{\mathbf{c}} E\right.$, F). (See Section 3.1 of [14] for gap.) Let $i \in S$. For each $a \in \mathcal{P a r}(\mathbf{c} E, \mathbf{F})$, we take $a^{\prime}(\epsilon, i) \in m^{-1} \cdot \mathbf{Z}$ such that $\left|a^{\prime}(\epsilon, i)-a\right|<m^{-1}$. Let $L(\epsilon, i) \in \mathbf{R}$ be determined by the following:

$$
L(\epsilon, i) \cdot \operatorname{rank}(E):=\sum\left(a^{\prime}(\epsilon, i)-a\right) \cdot \operatorname{rank}^{i} \mathrm{Gr}_{a}^{F}(\mathrm{c} E)
$$


Then we put $a(\epsilon, i):=a^{\prime}(\epsilon, i)-L(\epsilon, i)$ and $\varphi(a, k):=a(\epsilon, i)+k \cdot \epsilon$. By construction, we have the following equality:

$$
\sum_{a, k} \varphi(a, k) \cdot \operatorname{rank}\left({ }^{i} \mathrm{Gr}_{a, k}^{\widetilde{F}}(\mathrm{c} E)\right)=\sum_{a} a \cdot \operatorname{rank}\left({ }^{i} \mathrm{Gr}_{a}^{F}(\mathrm{c} E)\right)
$$

Hence, we have par-c ${ }_{1}(\mathbf{c} E, \mathbf{F})=\operatorname{par}_{-} \mathrm{c}_{1}\left(\mathbf{c} E, \mathbf{F}^{(\epsilon)}\right)$. For each $i$, we also have some $-1 / m<\gamma_{i} \leq 0$ such that

$$
\mathcal{P a r}\left({ }_{\mathbf{c}} E, \mathbf{F}^{(\epsilon)}, i\right) \subset\left\{c_{i}+\gamma_{i}+p / m \mid p \in \mathbf{Z}_{\leq 0},-1<\gamma_{i}+p / m \leq 0\right\} .
$$

In other words, $\left(\mathbf{c} E, \mathbf{F}^{(\epsilon)}, \mathbb{D}^{\lambda}\right)$ satisfies the SPW-condition (Definition 2.6).

Remark 2.23 The construction given in this subsection is valid, when the base manifold $X$ is a curve. However, some modification would be required in the case $\operatorname{dim} X \geq 3$, because a filtration at the generic point $D_{i}$ may not be extended to a filtration by vector bundles on $D_{i}$ if $\operatorname{dim} D_{i} \geq 2$.

\subsection{Generality for $\lambda$-connection in the $C^{\infty}$-category}

We give some generality for $\lambda$-connections in the $C^{\infty}$-category. They are straightforward generalizations of the argument for Higgs bundles or flat bundles given in Simpson's papers (for example [17; 19]), and hence we will often omit to give a detailed proof. For simplicity, we will assume $\lambda \neq 0$.

2.2.1 The induced operators Let $X$ be a complex manifold, and $\left(E, \mathbb{D}^{\lambda}\right)$ be a flat $\lambda$-connection on $X$. We have the decomposition of $\mathbb{D}^{\lambda}$ into the $(0,1)$-part $d_{E}^{\prime \prime}$ and the $(1,0)-$ part $d_{E}^{\prime}$. The holomorphic structure of $E$ is given by $d_{E}^{\prime \prime}$. Recall that the twisted Leibniz rule $d_{E}^{\prime}(f \cdot v)=\lambda \cdot \partial_{X}(f) v+f \cdot d_{E}^{\prime} v$ holds for $f \in C^{\infty}(X)$ and $v \in C^{\infty}(X, E)$. Let $h$ be a hermitian metric of $E$. From $d_{E}^{\prime \prime}$ and $h$, we obtain the $(1,0)$-operator $\delta_{E, h}^{\prime}$ determined by $\bar{\partial} h(u, v)=h\left(d_{E}^{\prime \prime} u, v\right)+h\left(u, \delta_{E, h}^{\prime} v\right)$. From $d_{E}^{\prime}$ and $h$, we obtain the $(0,1)$-operator $\delta_{E, h}^{\prime \prime}$ determined by $\lambda \partial h(u, v)=h\left(d_{E}^{\prime} u, v\right)+h\left(u, \delta_{E, h}^{\prime \prime} v\right)$. We remark that $\delta_{E, h}^{\prime \prime}(f \cdot v)=\bar{\lambda} \cdot \bar{\partial}_{X} f \cdot v+f \cdot \delta_{E, h}^{\prime \prime}(v)$. We obtain the operators

$$
\begin{aligned}
\bar{\partial}_{E, h} & :=\frac{1}{1+|\lambda|^{2}}\left(d_{E}^{\prime \prime}+\lambda \delta_{E, h}^{\prime \prime}\right), \quad \partial_{E, h}:=\frac{1}{1+|\lambda|^{2}}\left(\bar{\lambda} d_{E}^{\prime}+\delta_{E, h}^{\prime}\right), \\
\theta_{E, h}^{\dagger} & :=\frac{1}{1+|\lambda|^{2}}\left(\bar{\lambda} d_{E}^{\prime \prime}-\delta_{E, h}^{\prime \prime}\right), \quad \theta_{E, h}:=\frac{1}{1+|\lambda|^{2}}\left(d_{E}^{\prime}-\lambda \delta_{E, h}^{\prime}\right) .
\end{aligned}
$$

It is easy to see the following Leibniz rule:

$$
\bar{\partial}_{E, h}(f s)=\bar{\partial}_{X} f \cdot s+f \cdot \bar{\partial}_{E, h} s, \quad \partial_{E, h}(f s)=\partial_{X} f \cdot s+f \cdot \partial_{E, h} s .
$$


On the other hand, $\theta$ and $\theta^{\dagger}$ give the sections of $\operatorname{End}(E) \otimes \Omega^{1,0}$ and $\operatorname{End}(E) \otimes \Omega^{0,1}$ respectively. We also have the formulas:

$$
\begin{aligned}
d_{E}^{\prime \prime} & =\bar{\partial}_{E, h}+\lambda \theta_{E, h}^{\dagger}, & d_{E}^{\prime} & =\lambda \partial_{E, h}+\theta_{E, h}, \\
\delta_{E, h}^{\prime} & =\partial_{E, h}-\bar{\lambda} \theta_{E, h}, & \delta_{E, h}^{\prime \prime} & =\bar{\lambda} \bar{\partial}_{E, h}-\theta_{E, h}^{\dagger}
\end{aligned}
$$

Remark 2.24 The index " $E, h$ " is attached to emphasize the bundle $E$ and the metric $h$. We will often omit them if there are no risk of confusion.

Remark 2.25 We have the hermitian product $(\cdot, \cdot)_{h}:\left(E \otimes \Omega^{\cdot}\right) \otimes\left(E \otimes \Omega^{\prime}\right) \longrightarrow$ $\Omega$ induced by $h$. For a section $A$ of $\operatorname{End}(E) \otimes \Omega^{p, q}$, let $A_{h}^{\dagger}$ denote the section of $\operatorname{End}(E) \otimes \Omega^{q, p}$ which is the adjoint of $A$ with respect to $h$ in the sense $(A \cdot u, v)_{h}=\left(u, A_{h}^{\dagger} v\right)_{h}$. The above $\theta_{h}^{\dagger}$ is the adjoint of $\theta_{h}$ in this sense.

We put $\mathbb{D}_{h}^{\lambda \star}:=\delta_{h}^{\prime}-\delta_{h}^{\prime \prime}=\partial_{h}+\theta_{h}^{\dagger}-\bar{\lambda}\left(\bar{\partial}_{h}+\theta_{h}\right)$. We have the following formula:

$$
\bar{\partial}_{h}+\theta_{h}=\frac{\mathbb{D}^{\lambda}-\lambda \mathbb{D}_{h}^{\lambda \star}}{1+|\lambda|^{2}}, \quad \partial_{h}+\theta_{h}^{\dagger}=\frac{\mathbb{D}_{h}^{\lambda \star}+\bar{\lambda} \mathbb{D}^{\lambda}}{1+|\lambda|^{2}}
$$

We recall that $h$ is called a pluri-harmonic metric if $\left(\bar{\partial}_{h}+\theta_{h}\right)^{2}=0$ holds, ie, $\left(E, \bar{\partial}_{h}, \theta_{h}\right)$ is a Higgs bundle. The condition is equivalent to $\left[\mathbb{D}^{\lambda}, \mathbb{D}_{h}^{\lambda \star}\right]=0$. In the following, a $\lambda$-flat bundle with pluri-harmonic metric is called a harmonic bundle.

Let us consider the case in which $X$ is provided with a Kahler form $\omega$. For a differential operator $A$ of $E \otimes \Omega^{*}$ of degree one, ie, $A: C^{\infty}\left(X, E \otimes \Omega^{i}\right) \longrightarrow C^{\infty}\left(X, E \otimes \Omega^{i+1}\right)$, let $A^{*}$ denote a formal adjoint with respect to $\omega$ and $h$, ie, $\int_{X}(A u, v)_{h, \omega} \operatorname{dvol}_{\omega}=$ $\int_{X}\left(u, A^{*} v\right)_{h, \omega} \mathrm{dvol}_{\omega}$ hold for any $C^{\infty}$-sections $u$ and $v$ with compact supports. Here, $(\cdot, \cdot)_{h, \omega}$ denotes the Hermitian inner product of appropriate vector bundles induced by $h$ and $\omega$.

Lemma 2.26 $\left(\mathbb{D}^{\lambda \star}\right)^{*}=\sqrt{-1}\left[\Lambda_{\omega}, \mathbb{D}^{\lambda}\right]$ and $\left(\mathbb{D}^{\lambda}\right)^{*}=-\sqrt{-1}\left[\Lambda_{\omega}, \mathbb{D}^{\lambda \star}\right]$.

Proof It follows from the relations $\partial^{*}=\sqrt{-1}\left[\Lambda_{\omega}, \bar{\partial}_{E}\right], \bar{\partial}^{*}=-\sqrt{-1}\left[\Lambda_{\omega}, \partial_{E}\right], \theta^{*}=$ $-\sqrt{-1}\left[\Lambda_{\omega}, \theta^{\dagger}\right]$ and $\left(\theta^{\dagger}\right)^{*}=\sqrt{-1}\left[\Lambda_{\omega}, \theta\right]$.

The Laplacian $\Delta_{h, \omega}^{\lambda}: C^{\infty}(X, E) \longrightarrow C^{\infty}(X, E)$ is defined by

$$
\Delta_{h, \omega}^{\lambda}:=\sqrt{-1} \Lambda_{\omega} \mathbb{D}^{\lambda} \mathbb{D}^{\lambda \star} .
$$


Remark 2.27 For the differential operators of functions, we have

$$
\Delta_{\omega}^{\lambda}:=\sqrt{-1} \Lambda(\bar{\partial}+\lambda \partial) \circ(\partial-\bar{\lambda} \bar{\partial})=\left(1+|\lambda|^{2}\right) \sqrt{-1} \Lambda \bar{\partial} \partial=\left(1+|\lambda|^{2}\right) \Delta_{\omega}^{\prime \prime},
$$

where $\Delta_{\omega}^{\prime \prime}$ denotes the usual Laplacian $\sqrt{-1} \Lambda_{\omega} \bar{\partial} \partial$.

Lemma 2.28 When $\lambda \neq 0$, we have $\bar{\lambda}^{-1} \partial_{h}^{2}+\lambda^{-1} \theta_{h}^{2}=0$ and $\lambda^{-1} \bar{\partial}_{h}^{2}+\bar{\lambda}^{-1}\left(\theta_{h}^{\dagger}\right)^{2}=0$.

Proof From the flatness $\left(\mathbb{D}^{\lambda}\right)^{2}=0$, we obtain the following formulas:

$$
\begin{gathered}
\left(\bar{\partial}_{h}+\lambda \theta_{h}^{\dagger}\right)^{2}=\bar{\partial}_{h}^{2}+\lambda \bar{\partial}_{h} \theta_{h}^{\dagger}+\lambda^{2}\left(\theta_{h}^{\dagger}\right)^{2}=0 \\
\left(\lambda \partial_{h}+\theta_{h}\right)^{2}=\lambda^{2} \partial_{h}^{2}+\lambda \partial_{h} \theta_{h}+\theta_{h}^{2}=0 \\
{\left[\bar{\partial}_{h}+\lambda \theta_{h}^{\dagger}, \lambda \partial_{h}+\theta_{h}\right]=\lambda\left(\left[\bar{\partial}_{h}, \partial_{h}\right]+\left[\theta_{h}^{\dagger}, \theta_{h}\right]\right)+\bar{\partial}_{h} \theta_{h}+\lambda^{2} \partial_{h} \theta_{h}^{\dagger}=0}
\end{gathered}
$$

It is easy to see $\left(\bar{\partial}_{h}^{2}\right)_{h}^{\dagger}=-\partial_{h}^{2},\left(\bar{\partial}_{h} \theta_{h}^{\dagger}\right)^{\dagger}=\partial_{h} \theta_{h}$ and $\left(\theta_{h}^{\dagger}\right)^{2}=-\left(\theta_{h}^{2}\right)^{\dagger}$. Therefore, we obtain the following equality from (5):

$$
-\partial_{h}^{2}+\bar{\lambda}\left(\partial_{h} \theta_{h}\right)-\bar{\lambda}^{2} \theta_{h}^{2}=0
$$

From (6) and (8), we obtain $\left(\lambda+\bar{\lambda}^{-1}\right) \partial_{h}^{2}+\left(\lambda^{-1}+\bar{\lambda}\right) \theta_{h}^{2}=\left(1+|\lambda|^{2}\right)\left(\bar{\lambda}^{-1} \partial_{h}^{2}+\lambda^{-1} \theta_{h}^{2}\right)=$ 0 , which gives the first formula in the lemma. The second formula can be obtained by taking the adjoint.

Lemma 2.29 If $\lambda \neq 0$, the following holds:

$$
\bar{\lambda}^{-1} \cdot \partial_{h} \theta_{h}^{\dagger}+\lambda^{-1} \cdot \bar{\partial}_{h} \theta_{h}=0, \quad\left[\partial_{h}, \bar{\partial}_{h}\right]+\left[\theta_{h}, \theta_{h}^{\dagger}\right]=0
$$

Proof It is easy to check $\left[\partial_{h}, \bar{\partial}_{h}\right]_{h}^{\dagger}=-\left[\partial_{h}, \bar{\partial}_{h}\right],\left[\theta_{h}, \theta_{h}^{\dagger}\right]_{h}^{\dagger}=-\left[\theta_{h}, \theta_{h}^{\dagger}\right]$ and $\left(\bar{\partial}_{h} \theta_{h}\right)_{h}^{\dagger}=$ $\partial_{h} \theta_{h}^{\dagger}$. Hence, we obtain the following equality from (7):

$$
-\left[\bar{\partial}_{h}, \partial_{h}\right]-\left[\theta_{h}^{\dagger}, \theta_{h}\right]+\bar{\lambda}^{-1} \cdot \partial_{h} \theta_{h}^{\dagger}+\bar{\lambda} \cdot \bar{\partial}_{h} \theta_{h}=0 .
$$

The claim of the lemma immediately follows from (7) and (9).

Corollary 2.30 When $\lambda \neq 0$, the pluri-harmonicity of the metric $h$ is equivalent to the vanishings $\theta_{h}^{2}=0$ and $\bar{\partial}_{h} \theta_{h}=0$. 
2.2.2 Local expression Let $\left(E, \mathbb{D}^{\lambda}\right)$ be a flat $\lambda$-connection, and let $h$ be a $C^{\infty}$ metric. Let $\mathbf{v}=\left(v_{1}, \ldots, v_{r}\right)$ be a holomorphic frame of $E$. Let $H=H(h, \mathbf{v})$ denote the hermitian matrix valued function of $h$ with respect to $\mathbf{v}$, ie, $H_{i, j}=h\left(v_{i}, v_{j}\right)$. Let us look at the local expression of the induced operators.

Let $A$ denote the $M(r)$-valued $(1,0)$-form of $\mathbb{D}^{\lambda}$ with respect to $\mathbf{v}$, ie, $\mathbb{D}^{\lambda} \mathbf{v}=\mathbf{v} \cdot A$, in other words, $\mathbb{D}^{\lambda} v_{i}=\sum A_{j i} \cdot v_{j}$. Let $B$ denote the $(1,0)$-form of $\delta_{h}^{\prime}$ with respect to $\mathbf{v}$, ie, $\delta_{h}^{\prime} \mathbf{v}=\mathbf{v} \cdot B$, and then we have

$$
\bar{\partial} h\left(v_{i}, v_{j}\right)=h\left(v_{i}, \delta_{h}^{\prime} v_{j}\right)=\sum h\left(v_{i}, B_{k, j} v_{k}\right) .
$$

Hence, $\bar{\partial} H=H \cdot \bar{B}$, ie, we obtain $B=\bar{H}^{-1} \partial \bar{H}$. Let $C$ denote the $(0,1)$-form of $\delta_{h}^{\prime \prime}$ with respect to $\mathbf{v}$, ie, $\delta_{h}^{\prime \prime} \mathbf{v}=\mathbf{v} \cdot C$, and then we have

$$
\lambda \cdot \partial h\left(v_{i}, v_{j}\right)=h\left(d^{\prime} v_{i}, v_{j}\right)+h\left(v_{i}, \delta_{h}^{\prime \prime} v_{j}\right)=\sum_{k} h\left(A_{k, i} v_{k}, v_{j}\right)+\sum_{k} h\left(v_{i}, C_{k, j} v_{k}\right) .
$$

Hence, $\lambda \partial H={ }^{t} A H+H \bar{C}$, ie, we obtain $C=\bar{\lambda} \cdot \bar{H}^{-1} \bar{\partial} \bar{H}-\bar{H}^{-1 t} \bar{A} \bar{H}$. Thus, we obtain the following:

$$
\theta_{h} \mathbf{v}=\mathbf{v} \cdot \frac{1}{1+|\lambda|^{2}}\left(A-\lambda \bar{H}^{-1} \partial \bar{H}\right), \quad \bar{\partial}_{h} \mathbf{v}=\mathbf{v} \cdot \frac{\lambda}{1+|\lambda|^{2}}\left(\bar{\lambda} \cdot \bar{H}^{-1} \bar{\partial} \bar{H}-A_{h}^{\dagger}\right)
$$

Here, $A^{\dagger}$ denote the adjoint of $A$ with respect to $h$, ie, $A_{h}^{\dagger}=\bar{H}^{-1} \cdot{ }^{t} \bar{A} \cdot \bar{H}$.

2.2.3 Pseudo-curvature and a Hermitian-Einstein condition Assume $\lambda \neq 0$. For a flat $\lambda$-connection $\left(E, \mathbb{D}^{\lambda}\right)$ with a hermitian metric $h$, the pseudo-curvature $G\left(h, \mathbb{D}^{\lambda}\right)$ is defined as follows:

$$
G\left(h, \mathbb{D}^{\lambda}\right):=\left[\mathbb{D}^{\lambda}, \mathbb{D}_{h}^{\lambda \star}\right]=-\frac{\left(1+|\lambda|^{2}\right)^{2}}{\lambda}\left(\bar{\partial}_{h}+\theta_{h}\right)^{2}
$$

Then a hermitian metric $h$ is a pluri-harmonic metric for $\left(E, \mathbb{D}^{\lambda}\right)$, if and only if $G\left(h, \mathbb{D}^{\lambda}\right)=0$ holds. We will often use the symbols $G(h)$ or $G_{h}$ instead of $G\left(h, \mathbb{D}^{\lambda}\right)$ if there are no risk of confusion.

When $X$ is provided with a Kahler form $\omega$, a Hermitian-Einstein condition for $h$ is $\Lambda_{\omega} G\left(h, \mathbb{D}^{\lambda}\right)^{\perp}=0$, where “ $\perp$ ” means the trace free part.

2.2.4 Some relations between curvature and pseudo-curvature By the construction of $\delta_{h}^{\prime}$, the operator $d^{\prime \prime}+\delta_{h}^{\prime}$ is a unitary connection of $(E, h)$. The curvature of $d^{\prime \prime}+\delta_{h}^{\prime}$ is denoted by $R\left(d^{\prime \prime}, h\right)$. We have the following expression of $R\left(d^{\prime \prime}, h\right)$ due 
to $\left[d^{\prime \prime}, d^{\prime}\right]=0$ :

$$
\begin{aligned}
R\left(d^{\prime \prime}, h\right)=\left[d^{\prime \prime}, \delta_{h}^{\prime}\right] & =\left[d^{\prime \prime}, \lambda^{-1} d^{\prime}\right]-\frac{1+|\lambda|^{2}}{\lambda}\left[d^{\prime \prime}, \theta_{h}\right] \\
& =-\frac{1+|\lambda|^{2}}{\lambda}\left(\bar{\partial}_{h} \theta_{h}+\lambda\left[\theta_{h}^{\dagger}, \theta_{h}\right]\right)
\end{aligned}
$$

Lemma 2.31 The following equality holds:

$$
\operatorname{tr} R\left(d^{\prime \prime}, h\right)=\frac{1}{1+|\lambda|^{2}} \operatorname{tr} G\left(\mathbb{D}^{\lambda}, h\right)=-\frac{1+|\lambda|^{2}}{\lambda} \bar{\partial} \operatorname{tr} \theta_{h}
$$

Proof From (10), we obtain $\operatorname{tr} R\left(d^{\prime \prime}, h\right)=-\left(1+|\lambda|^{2}\right) \lambda^{-1} \cdot \bar{\partial} \operatorname{tr} \theta_{h}$. We have the following equality:

$$
\operatorname{tr} G\left(h, \mathbb{D}^{\lambda}\right)=-\frac{\left(1+|\lambda|^{2}\right)^{2}}{\lambda} \operatorname{tr}\left(\bar{\partial}_{h}^{2}+\bar{\partial}_{h} \theta_{h}+\theta_{h}^{2}\right)=-\frac{\left(1+|\lambda|^{2}\right)^{2}}{\lambda} \bar{\partial} \operatorname{tr} \theta_{h}
$$

Here, we have used $\operatorname{tr}\left(\theta_{h}^{2}\right)=0$, which implies $\operatorname{tr}\left(\bar{\partial}_{h}^{2}\right)=0$ due to Lemma 2.28. Thus we are done.

Lemma 2.32 In the case $\operatorname{dim} X=2$, we have the following formula:

$$
\operatorname{tr}\left(R\left(d^{\prime \prime}, h\right)^{2}\right)=\frac{1}{\left(1+|\lambda|^{2}\right)^{2}} \operatorname{tr}\left(G\left(h, \mathbb{D}^{\lambda}\right)^{2}\right)-\frac{\left(1+|\lambda|^{2}\right)^{2}}{\lambda} \bar{\partial} \operatorname{tr}\left(\theta_{h}^{2} \cdot \theta_{h}^{\dagger}\right)
$$

Proof We have the following equalities:

$$
\begin{gathered}
\operatorname{tr}\left(G\left(h, \mathbb{D}^{\lambda}\right)^{2}\right)=\frac{\left(1+|\lambda|^{2}\right)^{4}}{\lambda^{2}}\left(\operatorname{tr}\left(\left(\bar{\partial}_{h} \theta_{h}\right)^{2}\right)+2 \operatorname{tr}\left(\bar{\partial}_{h}^{2} \cdot \theta_{h}^{2}\right)\right) \\
\operatorname{tr}\left(R\left(h, d^{\prime \prime}\right)^{2}\right)=\frac{\left(1+|\lambda|^{2}\right)^{2}}{\lambda^{2}}\left(\operatorname{tr}\left(\left(\bar{\partial}_{h} \theta_{h}\right)^{2}\right)+2 \lambda \operatorname{tr}\left(\bar{\partial}_{h} \theta_{h} \cdot\left[\theta_{h}, \theta_{h}^{\dagger}\right]\right)+\lambda^{2} \operatorname{tr}\left(\left[\theta_{h}, \theta_{h}^{\dagger}\right]^{2}\right)\right)
\end{gathered}
$$

Because $\operatorname{tr}\left(\left[\theta_{h}, \theta_{h}^{\dagger}\right]^{2}\right)=-2 \operatorname{tr}\left(\theta_{h}^{2} \theta_{h}^{\dagger}{ }^{2}\right)$ and $\left(\bar{\partial}_{h}+\lambda \theta_{h}^{\dagger}\right)^{2}=\bar{\partial}_{h}^{2}+\lambda \bar{\partial}_{h} \theta_{h}^{\dagger}+\lambda^{2} \theta_{h}^{\dagger 2}=0$, we obtain the following:

$$
\lambda^{2} \operatorname{tr}\left(\left[\theta_{h}, \theta_{h}^{\dagger}\right]^{2}\right)=-2 \operatorname{tr}\left(\lambda^{2} \cdot \theta_{h}^{2} \cdot \theta_{h}^{\dagger 2}\right)=2 \operatorname{tr}\left(\bar{\partial}_{h}^{2} \cdot \theta_{h}^{2}+\lambda \cdot \bar{\partial}_{h} \theta_{h}^{\dagger} \cdot \theta_{h}^{2}\right)
$$

Hence, we have the following equality:

$$
\begin{aligned}
\operatorname{tr}\left(R\left(h, d^{\prime \prime}\right)^{2}\right) & =\left(\frac{1+|\lambda|^{2}}{\lambda}\right)^{2} \times \\
& \left(\operatorname{tr}\left(\left(\bar{\partial}_{h} \theta_{h}\right)^{2}\right)+2 \lambda \operatorname{tr}\left(\bar{\partial}_{h} \theta_{h} \cdot\left[\theta_{h}, \theta_{h}^{\dagger}\right]\right)+2 \operatorname{tr}\left(\bar{\partial}_{h}^{2} \cdot \theta_{h}^{2}\right)+2 \lambda \operatorname{tr}\left(\bar{\partial}_{h} \theta_{h}^{\dagger} \cdot \theta_{h}^{2}\right)\right)
\end{aligned}
$$


We also remark the following:

$$
\begin{aligned}
\operatorname{tr}\left(\bar{\partial}_{h} \theta_{h} \cdot\left[\theta_{h}, \theta_{h}^{\dagger}\right]\right)+ & \operatorname{tr}\left(\theta_{h}^{2} \cdot \bar{\partial}_{h} \theta_{h}^{\dagger}\right) \\
& =\operatorname{tr}\left(\left(\bar{\partial}_{h} \theta_{h}\right) \cdot \theta_{h} \cdot \theta_{h}^{\dagger}\right)+\operatorname{tr}\left(\bar{\partial}_{h} \theta_{h} \cdot \theta_{h}^{\dagger} \cdot \theta_{h}\right)-\operatorname{tr}\left(\theta_{h} \cdot \bar{\partial}_{h} \theta_{h}^{\dagger} \cdot \theta_{h}\right) \\
& =\bar{\partial} \operatorname{tr}\left(\theta_{h} \cdot \theta_{h}^{\dagger} \cdot \theta_{h}\right)=-\bar{\partial} \operatorname{tr}\left(\theta_{h}^{2} \cdot \theta_{h}^{\dagger}\right)
\end{aligned}
$$

Then the claim of the lemma immediately follows.

2.2.5 Change of hermitian metrics Let $h_{i}(i=1,2)$ be hermitian metrics of $E$. The endomorphism $s$ is determined by $h_{2}=h_{1} \cdot s$, ie, $h_{2}(u, v)=h_{1}(s \cdot u, v)=$ $h_{1}(u, s \cdot v)$, which is self-adjoint with respect to both $h_{1}$ and $h_{2}$. Then we have the relations $\delta_{h_{2}}^{\prime}=\delta_{h_{1}}^{\prime}+s^{-1} \delta_{h_{1}}^{\prime} s$ and $\delta_{h_{2}}^{\prime \prime}=\delta_{h_{1}}^{\prime \prime}+s^{-1} \delta_{h_{1}}^{\prime \prime} s$. Therefore, we have the following relations from (4):

$$
\begin{array}{ll}
\bar{\partial}_{h_{2}}=\bar{\partial}_{h_{1}}+\frac{\lambda}{1+|\lambda|^{2}} s^{-1} \delta_{h_{1}}^{\prime \prime} s, & \partial_{h_{2}}=\partial_{h_{1}}+\frac{1}{1+|\lambda|^{2}} s^{-1} \delta_{h_{1}}^{\prime} s \\
\theta_{h_{2}}^{\dagger}=\theta_{h_{1}}^{\dagger}-\frac{1}{1+|\lambda|^{2}} s^{-1} \delta_{h_{2}}^{\prime \prime} s, & \theta_{h_{2}}=\theta_{h_{1}}-\frac{\lambda}{1+|\lambda|^{2}} s^{-1} \delta_{h_{1}}^{\prime} s
\end{array}
$$

We also have $\mathbb{D}_{h_{2}}^{\lambda \star}=\mathbb{D}_{h_{1}}^{\lambda \star}+s^{-1} \mathbb{D}_{h_{1}}^{\lambda \star} s$, and thus

$$
\left[\mathbb{D}^{\lambda}, \mathbb{D}_{h_{2}}^{\lambda \star}\right]=\left[\mathbb{D}^{\lambda}, \mathbb{D}_{h_{1}}^{\lambda \star}\right]+\mathbb{D}^{\lambda}\left(s^{-1}\right) \cdot \mathbb{D}_{h_{1}}^{\lambda \star} s+s^{-1} \mathbb{D}^{\lambda} \mathbb{D}_{h_{1}}^{\lambda \star} s
$$

Then we obtain the following formula:

$$
\Delta_{h_{1}, \omega}^{\lambda} s=s \sqrt{-1}\left(\Lambda_{\omega} G\left(h_{2}\right)-\Lambda_{\omega} G\left(h_{1}\right)\right)+\sqrt{-1} \Lambda_{\omega}\left(\mathbb{D}^{\lambda} s \cdot s^{-1} \mathbb{D}^{\lambda \star} s\right)
$$

In particular, we obtain the following formula by taking the trace:

$$
\Delta_{\omega}^{\lambda} \operatorname{tr}(s)=\operatorname{tr}\left(s \sqrt{-1}\left(\Lambda_{\omega} G\left(h_{2}\right)-\Lambda_{\omega} G\left(h_{1}\right)\right)\right)-\left|\mathbb{D}^{\lambda}(s) s^{-1 / 2}\right|_{h_{1}, \omega}^{2}
$$

As in Lemma 3.1 of [17], we can derive the following inequality:

$$
\Delta_{\omega}^{\lambda} \log \operatorname{tr}(s) \leq\left|\Lambda_{\omega} G\left(h_{1}\right)\right|_{h_{1}}+\left|\Lambda_{\omega} G\left(h_{2}\right)\right|_{h_{2}}
$$

\subsection{Analytic stability of $\lambda$-flat bundle}

Let $X$ be a complex manifold with a Kahler form $\omega$. We often impose the following condition on them as in [17]. 


\section{Condition 2.33}

(1) The volume of $X$ with respect to $\omega$ is finite.

(2) There exists a $C^{\infty}$-function $\phi: X \longrightarrow \mathbf{R}_{\geq 0}$ with the following properties:

- $\{x \in X \mid \phi(x) \leq a\}$ is compact for any $a$.

- $0 \leq \sqrt{-1} \partial \bar{\partial} \phi \leq C \cdot \omega$, and $\bar{\partial} \phi$ is bounded with respect to $\omega$.

(3) There exists a continuous increasing function $a:[0, \infty[\longrightarrow[0, \infty[$ with the following properties:

- $a(0)=0$ and $a(t)=t$ for $t \geq 1$.

- Let $f$ be a positive bounded function on $X$ such that $\Delta_{\omega} f \leq B$ for some $B \in \mathbf{R}$. Then there exists a constant $C(B)$, depending only on $B$, such that $\sup _{X}|f| \leq C(B) \cdot a\left(\int_{X}|f| \cdot \operatorname{dvol}_{\omega}\right)$. Moreover, $\Delta_{\omega}(f) \leq 0$ implies $\Delta_{\omega}(f)=0$.

Let $\left(E, \mathbb{D}^{\lambda}\right)$ be a $\lambda$-flat bundle on $X$. There are two kinds of finiteness conditions for the pseudo-curvature of $\left(E, \mathbb{D}^{\lambda}, h\right)$. The stronger one is

$$
\sup \left|G\left(h, \mathbb{D}^{\lambda}\right)\right|_{h, \omega}<\infty .
$$

It implies the weaker one

$$
\sup \left|\Lambda_{\omega} G\left(h, \mathbb{D}^{\lambda}\right)\right|_{h, \omega}<\infty
$$

When we are given a hermitian metric $h$ of $E$ satisfying the finiteness (14), the degree $\operatorname{deg}_{\omega}(E, h)$ is defined as follows:

$$
\operatorname{deg}_{\omega}(E, h):=\frac{\sqrt{-1}}{2 \pi} \int_{X} \frac{\operatorname{tr} G\left(h, \mathbb{D}^{\lambda}\right)}{1+|\lambda|^{2}} \omega^{n-1}=\frac{\sqrt{-1}}{2 \pi} \int_{X} \operatorname{tr} R\left(h, d^{\prime \prime}\right) \cdot \omega^{n-1}
$$

Here, we have used (11). For any $\lambda$-flat subbundle $\left(V, \mathbb{D}_{V}^{\lambda}\right) \subset\left(E, \mathbb{D}^{\lambda}\right)$, the restriction $h_{V}:=h_{\mid V}$ induces $\operatorname{deg}_{\omega}\left(V, h_{V}\right)$. As in Lemma 3.2 of [17], we have the Chern-Weil formula. The proof is done in the same way.

Lemma 2.34 Assume that (14) is satisfied. Let $\pi_{V}$ denote the orthogonal projection of $E$ onto $V$. Then the following equality holds:

$$
\operatorname{deg}_{\omega}\left(V, h_{V}\right)=\frac{1}{2 \pi} \frac{1}{1+|\lambda|^{2}}\left(\sqrt{-1} \int_{X} \operatorname{tr}\left(\pi_{V} \circ G\left(h, \mathbb{D}^{\lambda}\right)\right) \cdot \omega^{n-1}-\int_{X}\left|\mathbb{D}^{\lambda} \pi_{V}\right|_{h, \omega}^{2}\right)
$$

The value is finite or $-\infty$. 
Definition $2.35\left(E, \mathbb{D}^{\lambda}, h\right)$ is defined to be analytically stable with respect to $\omega$, if the inequality

$$
\frac{\operatorname{deg}_{\omega}\left(V, h_{V}\right)}{\operatorname{rank} V}<\frac{\operatorname{deg}_{\omega}(E, h)}{\operatorname{rank} E}
$$

holds for any $\left(V, \mathbb{D}_{V}^{\lambda}\right) \subset\left(E, \mathbb{D}^{\lambda}\right)$ such that $0<\operatorname{rank} V<\operatorname{rank} E$.

\subsection{Review of Donaldson functional}

We recall the Donaldson functional by following Donaldson [4] and Simpson [17] in our situation. We give a rather detailed review because we would like to use variants (Lemma 2.45 and Lemma 2.48) of a main estimate (Proposition 2.41). They will be used in the proof of Propositions 4.1 and 4.2, together with Lemma 2.50.

2.4.1 Functions of self-adjoint endomorphisms Let $V$ be a vector space over $\mathbf{C}$ with a hermitian metric $h$. Let $S(V, h)$ denote the set of endomorphisms of $V$ which are self-adjoint with respect to $h$. Let $\varphi: \mathbf{R} \longrightarrow \mathbf{R}$ be a continuous function. Then $\varphi(s)$ is naturally defined for any $s \in S(V, h)$. Namely, let $v_{1}, \ldots, v_{r}$ be an orthogonal base which consists of eigen vectors of $s$, and let $v_{1}^{\vee}, \ldots, v_{r}^{\vee}$ be the dual base. Then we have the description $s=\sum \kappa_{i} \cdot v_{i}^{\vee} \otimes v_{i}$, and we put $\varphi(s):=\sum \varphi\left(\kappa_{i}\right) \cdot v_{i}^{\vee} \otimes v_{i}$. Thus, we obtain the induced map $\varphi: S(V, h) \longrightarrow S(V, h)$, which is well known to be continuous. To see the continuity, for example, we can argue as follows: Let $U(h)$ denote the unitary group with respect to $h$. Take $\mathbf{e}=\left(e_{1}, \ldots, e_{r}\right)$ be an orthogonal base of $V$. Let $T$ denote the set of endomorphisms of $V$ which is diagonal with respect to the base $\mathbf{e}$. Then we have the continuous surjective map $\pi: U(h) \times T \longrightarrow S(V, h)$ given by $(u, t) \longmapsto u \cdot t \cdot u^{-1}$. It is easy to check the continuity of the composite $\varphi \circ \pi$. Since the topology of $S(V, h)$ is the same as the induced topology via $\pi$, we obtain the continuity. When $\varphi$ is real analytic given by a convergent power series $\sum a_{j} \cdot t^{j}$, then $\varphi(s)=\sum a_{j} \cdot s^{j}$. The induced map is real analytic in this case.

Let $\Psi: \mathbf{R} \times \mathbf{R} \longrightarrow \mathbf{R}$ be a continuous function. For a self-adjoint map $s \in S(V, h)$, let $v_{1}, \ldots, v_{r}$ and $v_{1}^{\vee}, \ldots, v_{r}^{\vee}$ be as above. Then we put $\Psi(s)(A)=\sum \Psi\left(\kappa_{i}, \kappa_{j}\right) A_{i, j} v_{i}^{\vee} \otimes$ $v_{j}$ for any endomorphism $A=\sum A_{i, j} \cdot v_{i}^{\vee} \otimes v_{j}$ of $V$. Thus, we obtain $\Psi: S(V, h) \longrightarrow$ $S(\operatorname{End}(V), h)$, which is also well known to be continuous. Here, $S(\operatorname{End}(V), h)$ denotes the set of self-adjoint endomorphisms of $\operatorname{End}(V)$ with respect to the metric induced by $h$. To see the continuity, we can use the same argument as above. When $\Psi$ is real analytic given by a power series $\sum b_{m, n} t_{1}^{m} t_{2}^{n}$, then we have $\Psi(s)(A)=\sum b_{m, n} s^{m}$. $A \cdot s^{n}$, and the induced map is real analytic.

Let $\varphi: \mathbf{R} \longrightarrow \mathbf{R}$ be $C^{1}$, and let $d \varphi: \mathbf{R}^{2} \longrightarrow \mathbf{R}^{2}$ denote the continuous function given by $d \varphi\left(t_{1}, t_{2}\right)=\left(t_{1}-t_{2}\right)^{-1}\left(\varphi\left(t_{1}\right)-\varphi\left(t_{2}\right)\right)\left(t_{1} \neq t_{2}\right)$ and $d \varphi\left(t_{1}, t_{1}\right)=\varphi^{\prime}\left(t_{1}\right)$. In this 
case, the induced map $\varphi: S(V, h) \longrightarrow S(V, h)$ is also $C^{1}$, and the derivative at $s$ is given by $d \varphi(s)$. To see it, we can argue as follows: If $\varphi$ is real analytic, the claim can be checked by a direct calculation. In general, we can take an approximate sequence $\varphi_{i} \longrightarrow \varphi$ by real analytic functions on an appropriate compact neighbourhoods of the eigenvalues of $s \in S(V, h)$. The induced maps $\varphi_{i}: S(V, h) \longrightarrow S(V, h)$ and $d \varphi_{i}: S(V, h) \longrightarrow S(\operatorname{End}(V), h)$ uniformly converge to $\varphi$ and $d \varphi$ on an appropriate compact neighbourhoods of $s$. Then we can derive that $\varphi$ is the integral of the form $d \varphi$ by a general fact.

The construction can be done on manifolds. Namely, let $E$ be a $C^{\infty}$-vector bundle with a hermitian metric $h$. Let $S_{h}(E)$ (or simply $S_{h}$ ) be the bundle of selfadjoint endomorphisms of $(E, h)$, and let $S_{h}(\operatorname{End}(E))$ be the bundle of self-adjoint endomorphisms of $(\operatorname{End}(E), h)$. Then a continuous function $\varphi: \mathbf{R} \longrightarrow \mathbf{R}$ induces $\varphi: S_{h}(E) \longrightarrow S_{h}(E)$, and $\Psi: \mathbf{R}^{2} \longrightarrow \mathbf{R}$ induces $\Psi: S_{h}(E) \longrightarrow S_{h}(\operatorname{End}(E))$. We have $\mathbb{D}^{\lambda} \varphi(s)=d \varphi(s)\left(\mathbb{D}^{\lambda} s\right)$, if $\varphi$ is $C^{1}$.

2.4.2 A closed one form Let $(X, \omega)$ and $\left(E, \mathbb{D}^{\lambda}\right)$ be as in Condition 2.33. Following Simpson [17], we introduce the space $P\left(S_{h}\right)$ which consists of sections $s$ of $S_{h}(E)$ satisfying the following finiteness:

$$
\|s\|_{h, \omega, P}:=\sup _{X}|s|_{h}+\left\|\mathbb{D}^{\lambda} s\right\|_{2, h, \omega}+\left\|\Delta_{h, \omega}^{\lambda} s\right\|_{1, h, \omega}<\infty .
$$

Here, $\|\cdot\|_{p, h, \omega}$ denote the $L^{p}$-norm with respect to $(h, \omega)$. We will omit to denote $\omega$ and $h$, when there are no risk of confusion. The following lemma is the counterpart of Proposition 4.1 (d) in [17]. The proof is done in the same way.

Lemma 2.36 Suppose $\varphi$ and $\Psi$ are analytic functions on $\mathbf{R}$ with infinite radius of convergence. Then $\varphi: P\left(S_{h}\right) \longrightarrow P\left(S_{h}\right)$ and $\Psi: P\left(S_{h}\right) \longrightarrow P\left(S_{h}(\operatorname{End}(E))\right)$ are analytic.

Let $h$ be a metric satisfying (14). Let $\mathcal{P}_{+}\left(S_{h}\right)$ denote the set of self-adjoint positive definite endomorphisms $s$ with respect to $h$ such that $\|s\|_{h, P}<\infty$ and $\left\|s^{-1}\right\|_{h, P}<\infty$. Note $\|s\|_{h, P}<\infty$ and sup $\left|s^{-1}\right|_{h}<\infty$ imply $\left\|s^{-1}\right\|_{h, P}<\infty$. We put $\mathcal{P}_{h}:=\{h \cdot s \mid s \in$ $\left.\mathcal{P}_{+}\left(S_{h}\right)\right\}$. It is easy to see that any $h_{1} \in \mathcal{P}_{h}$ also satisfies (14) due to (12). It is also easy to see $\mathcal{P}_{h}=\mathcal{P}_{h_{1}}$ for $h_{1} \in \mathcal{P}_{h}$.

Let $\mathcal{P}\left(S_{h}\right)$ denote the space of self-adjoint endomorphisms $s$ with respect to $h$ such that $\|s\|_{P, h}<\infty$. It is easy to see that $\mathcal{P}_{+}\left(S_{h}\right)$ is open in $\mathcal{P}\left(S_{h}\right)$. In particular, we obtain the Banach manifold structure of $\mathcal{P}_{+}\left(S_{h}\right)$. By the natural bijection $\mathcal{P}_{h} \simeq \mathcal{P}_{+}\left(S_{h_{1}}\right)$ for $h_{1} \in \mathcal{P}_{h}$, we also obtain a Banach manifold structure of $\mathcal{P}_{h}$, which is independent of the choice of $h_{1} \in \mathcal{P}_{h}$. We have the map $\mathcal{P}\left(S_{h_{1}}\right) \longrightarrow \mathcal{P}_{+}\left(S_{h_{1}}\right)$ given by $s \longmapsto e^{s}$ 
(Lemma 2.36). It gives a diffeomorphism around $0 \in \mathcal{P}\left(S_{h_{1}}\right)$ and $1 \in \mathcal{P}_{+}\left(S_{h_{1}}\right)$. Therefore, the map $\mathcal{P}\left(S_{h_{1}}\right) \longrightarrow \mathcal{P}_{h}$ by $s \longmapsto h_{1} \cdot e^{s}$ gives a diffeomorphism around 0 and $h_{1}$. In particular, the tangent space $T_{h_{1}} \mathcal{P}_{h}$ can be naturally identified with $\mathcal{P}\left(S_{h_{1}}\right)$ for any $h_{1} \in \mathcal{P}_{h}$. We also have the natural isomorphism $\mathcal{P}\left(S_{h_{1}}\right) \simeq \mathcal{P}\left(S_{h}\right)$ given by $t \longmapsto u \cdot t$ for $h_{1}=h \cdot u \in \mathcal{P}_{h}$, which gives a local trivialization of the tangent bundle.

For any $h_{1} \in \mathcal{P}_{h}$ and $s \in T_{h_{1}} \mathcal{P}_{h}$, we put

$$
\Phi_{h_{1}}(s):=\int_{X} \Phi_{h_{1}}^{\prime}(s) \operatorname{dvol}_{\omega} \in \mathbf{C}, \quad \Phi_{h_{1}}^{\prime}(s):=\sqrt{-1} \operatorname{tr}\left(s \cdot \Lambda_{\omega} G\left(\mathbb{D}^{\lambda}, h_{1}\right)\right) .
$$

Then $\Phi^{\prime}$ gives an $L^{1}\left(X, \Omega_{X}^{1,1}\right)$-valued one form on $\mathcal{P}_{h}$, and $\Phi$ gives a one form of $\mathcal{P}_{h}$. The differentiability of $\Phi$ is easy to see.

Lemma 2.37 $\Phi$ is a closed one form.

Proof In the following argument, we use the symbol $\mathbb{D}^{\lambda \star}$ instead of $\mathbb{D}_{h}^{\lambda \star}$. Let $k_{1}, k_{2} \in \mathcal{P}_{h}$. They naturally give the vector field by addition. At any point $h_{1} \in \mathcal{P}_{h}$, they give the tangent vectors $\sigma=h_{1}^{-1} k_{1}$ and $\tau=h_{1}^{-1} k_{2}$ in $T_{h_{1}} \mathcal{P}_{h}=\mathcal{P}\left(S_{h_{1}}\right)$. Hence, the following holds at $h+\epsilon k_{1}$ :

$$
\Phi_{h+\epsilon k_{1}}\left(k_{2}\right)=\sqrt{-1} \int \operatorname{tr}\left(\left(h+\epsilon k_{1}\right)^{-1} \cdot k_{2} \cdot G\left(h+\epsilon k_{1}\right)\right) \cdot \omega^{n-1}
$$

We have $\left(h+\epsilon k_{1}\right)^{-1} k_{2}=(1+\epsilon \sigma)^{-1} \tau=\tau-\epsilon \sigma \tau+(1+\epsilon \sigma)^{-2} \epsilon^{2} \sigma^{2} \tau$. Remark $\sigma^{2} \tau$ is bounded. We also have the following:

$$
\begin{aligned}
(1+\epsilon \sigma)\left(G\left(h+\epsilon k_{1}\right)-\right. & G(h)) \\
& =\mathbb{D}^{\lambda} \mathbb{D}^{\lambda \star}(1+\epsilon \sigma)-\mathbb{D}^{\lambda}(1+\epsilon \sigma) \cdot(1+\epsilon \sigma)^{-1} \mathbb{D}^{\lambda \star}(1+\epsilon \sigma) \\
& =\epsilon \mathbb{D}^{\lambda} \mathbb{D}^{\lambda \star} \sigma-\epsilon^{2} \mathbb{D}^{\lambda} \sigma \cdot(1+\epsilon \sigma)^{-1} \mathbb{D}^{\lambda \star} \sigma .
\end{aligned}
$$

Hence, we have $G\left(h+\epsilon k_{1}\right)-G(h)=\epsilon \mathbb{D}^{\lambda} \mathbb{D}^{\lambda \star} \sigma+\epsilon^{2} R_{0}(\epsilon, \sigma, \tau)$, where $R_{0}(\epsilon, \sigma, \tau)$ is an $L^{1}$-section of $\operatorname{End}(E) \otimes \Omega^{2}$, and the $L^{1}$-norm is bounded independently from $\epsilon$. 
Therefore, we obtain the following:

$$
\begin{aligned}
\Phi_{h+\epsilon k_{1}}\left(k_{2}\right)-\Phi_{h}\left(k_{2}\right) \\
=\sqrt{-1} \int \operatorname{tr}\left(\left(h+\epsilon k_{1}\right)^{-1} k_{2} G\left(h+\epsilon k_{1}\right)\right) \omega^{n-1}-\sqrt{-1} \int \operatorname{tr}\left(h^{-1} k_{2} G(h)\right) \omega^{n-1} \\
=\sqrt{-1} \int \operatorname{tr}\left(\tau G\left(h+\epsilon k_{1}\right)-\tau G(h)\right) \omega^{n-1} \\
\quad-\epsilon \sqrt{-1} \int \operatorname{tr}\left(\sigma \tau G\left(h+\epsilon k_{1}\right)\right) \omega^{n-1}+\epsilon R_{1}(\epsilon, \sigma, \tau) \\
=\epsilon\left(\sqrt{-1} \int \operatorname{tr}\left(\tau \mathbb{D}^{\lambda} \mathbb{D}^{\lambda \star} \sigma\right) \cdot \omega^{n-1}-\sqrt{-1} \int \operatorname{tr}(\sigma \tau G(h)) \cdot \omega^{n-1}\right)+\epsilon R_{2}(\epsilon, \sigma, \tau)
\end{aligned}
$$

Here, we have $R_{i}(\epsilon, \sigma, \tau) \longrightarrow 0(i=1,2)$ as $\epsilon \rightarrow 0$, due to $\|\sigma\|_{P}<\infty$ and $\|\tau\|_{P}<\infty$. Hence, we obtain the following equality:

$$
d_{h} \Phi(\sigma, \tau)=\sqrt{-1} \int\left(\operatorname{tr}\left(\tau \mathbb{D}^{\lambda} \mathbb{D}^{\lambda \star} \sigma\right)-\operatorname{tr}\left(\sigma \mathbb{D}^{\lambda} \mathbb{D}^{\lambda \star} \tau\right)-\operatorname{tr}([\sigma, \tau] G(h)) \omega^{n-1}\right.
$$

We have the following equality, due to $\left[\mathbb{D}^{\lambda}, \mathbb{D}^{\lambda \star}\right]=G(h)$ :

$$
\begin{aligned}
(-\bar{\lambda} \bar{\partial}+\partial) & \operatorname{tr}\left(\tau \mathbb{D}^{\lambda} \sigma\right)+(\lambda \partial+\bar{\partial}) \operatorname{tr}\left(\sigma \mathbb{D}^{\lambda \star} \tau\right) \\
= & \operatorname{tr}\left(\mathbb{D}^{\lambda \star} \tau \mathbb{D}^{\lambda} \sigma\right)+\operatorname{tr}\left(\tau \mathbb{D}^{\lambda \star} \mathbb{D}^{\lambda} \sigma\right)+\operatorname{tr}\left(\mathbb{D}^{\lambda} \sigma \mathbb{D}^{\lambda \star} \tau\right)+\operatorname{tr}\left(\sigma \mathbb{D}^{\lambda} \mathbb{D}^{\lambda \star} \tau\right) \\
& =-\operatorname{tr}\left(\tau \mathbb{D}^{\lambda} \mathbb{D}^{\lambda \star} \sigma\right)+\operatorname{tr}(\tau \cdot[G(h), \sigma])+\operatorname{tr}\left(\sigma \mathbb{D}^{\lambda} \mathbb{D}^{\lambda \star} \tau\right) \\
& =-\operatorname{tr}\left(\tau \mathbb{D}^{\lambda} \mathbb{D}^{\lambda \star} \sigma\right)+\operatorname{tr}\left(\sigma \mathbb{D}^{\lambda} \mathbb{D}^{\lambda \star} \tau\right)+\operatorname{tr}([\sigma, \tau] \cdot G(h))
\end{aligned}
$$

Hence, we obtain $d_{h} \Phi(\sigma, \tau)=-\sqrt{-1} \int_{X}\left((-\bar{\lambda} \bar{\partial}+\partial) \operatorname{tr}\left(\tau \mathbb{D}^{\lambda} \sigma\right)+(\lambda \partial+\bar{\partial}) \operatorname{tr}\left(\sigma \mathbb{D}^{\lambda \star} \tau\right)\right)$. $\omega^{n-1}$. By using $\|\sigma\|_{P}<\infty$ and $\|\tau\|_{P}<\infty$, we obtain the vanishing of $d_{h} \Phi(\sigma, \tau)$, due to Lemma 5.2 of [17].

2.4.3 Donaldson functional For $h_{1}, h_{2} \in \mathcal{P}_{h}$, take a differentiable path $\gamma:[0,1] \longrightarrow$ $\mathcal{P}_{h}$ such that $\gamma(0)=h_{1}$ and $\gamma(1)=h_{2}$, and the Donaldson functional is defined to be

$$
M\left(h_{1}, h_{2}\right):=\int_{\gamma} \Phi .
$$

It is independent of the choice of a base metric $\omega$, in the case $\operatorname{dim} X=1$. We have $M\left(h_{1}, h_{2}\right)+M\left(h_{2}, h_{3}\right)=M\left(h_{1}, h_{3}\right)$ by the construction.

Lemma 2.38 When $h_{2}=h_{1} \cdot e^{s}$ for $s \in \mathcal{P}\left(S_{h_{1}}\right)$, we have the following formula:

$$
M\left(h_{1}, h_{2}\right)=\sqrt{-1} \int_{X} \operatorname{tr}\left(s \Lambda_{\omega} G\left(h_{1}\right)\right) \operatorname{dvol}_{\omega}+\int_{X}\left(\Psi(s) \mathbb{D}^{\lambda} s, \mathbb{D}^{\lambda} s\right)_{\omega, h_{1}} \mathrm{dvol}_{\omega}
$$


Here, $(\cdot, \cdot)_{\omega, h_{1}}$ denotes the hermitian product induced by $\omega$ and $h_{1}$, and $\Psi$ is given as follows:

$$
\Psi\left(t_{1}, t_{2}\right)=\frac{e^{t_{2}-t_{1}}-\left(t_{2}-t_{1}\right)-1}{\left(t_{2}-t_{1}\right)^{2}}
$$

See Section 2.4.1 for the meaning of $\Psi(s)\left(\mathbb{D}^{\lambda} s\right)$.

Proof Let $M^{\prime}\left(h_{1}, h_{2}\right)$ denote the right hand side of (15). The following formula immediately follows from the definition:

$$
\frac{\partial}{\partial u} M^{\prime}\left(h_{1} e^{t s}, h_{1} e^{(t+u) s}\right)_{\mid u=0}=\int_{X} \sqrt{-1} \operatorname{tr}\left(s \Lambda_{\omega} G\left(h_{1} e^{t s}\right)\right) .
$$

We also have the following equalities:

$$
\begin{aligned}
\frac{\partial^{2}}{\partial t \partial u} M^{\prime}\left(h_{1} e^{t s}, h_{1} e^{(t+u) s}\right)_{\mid u=0} & =\frac{\partial^{2}}{\partial t^{2}} M^{\prime}\left(h_{1}, h_{1} e^{t s}\right)_{\mid u=0} \\
& =\frac{\partial^{2}}{\partial t \partial u} M^{\prime}\left(h_{1}, h_{1} e^{(t+u) s}\right)_{\mid u=0} .
\end{aligned}
$$

The second equality can be shown formally. The first equality can be shown by the argument on page 883 of [17]. We also have the obvious equality:

$$
\frac{\partial}{\partial u} M^{\prime}\left(h_{1} e^{t s}, h_{1} e^{(t+u) s}\right)_{\mid t=0, u=0}=\frac{\partial}{\partial u} M^{\prime}\left(h_{1}, h_{1} e^{(t+u) s}\right)_{\mid t=0, u=0} .
$$

Hence, we obtain the following:

$$
\frac{\partial}{\partial t} M^{\prime}\left(h_{1}, h_{1} e^{t s}\right)=\int_{X} \sqrt{-1} \operatorname{tr}\left(s \Lambda_{\omega} G\left(h_{1} e^{t s}\right)\right) .
$$

Thus, $M^{\prime}\left(h_{1}, h_{1} e^{s}\right)$ is the integral of $\Phi^{\prime}$ along the path $\gamma(t)=h_{1} e^{t s}$, and hence $M^{\prime}\left(h_{1}, h_{2}\right)=M\left(h_{1}, h_{2}\right)$.

Remark 2.39 In [17], the formula (15) is adopted to be the definition of the functional. We follow the original definition due to Donaldson [4].

We obtain the following corollary due to the positivity of the function $\Psi$.

Corollary 2.40 If sup $\left|\Lambda_{\omega} G(h)\right|_{h}<B$ is satisfied, we have the following inequality:

$$
M\left(h, h e^{s}\right) \geq \sqrt{-1} \int \operatorname{tr}\left(s \Lambda_{\omega} G(h)\right) \cdot \operatorname{dvol}_{\omega} \geq-B \int|s|_{h} \cdot \operatorname{dvol}_{\omega} .
$$

In particular, the upper bound of $s$ gives the lower bound of $M\left(h, h e^{s}\right)$. 
2.4.4 Main estimate The following key estimate is the counterpart of Proposition 5.3 in [17]. The proof is the same.

Proposition 2.41 Fix $B>0$. Let $\left(E, \mathbb{D}^{\lambda}\right)$ be a flat $\lambda$-connection. Let $h$ be a hermitian metric of $E$ such that $\sup \left|\Lambda_{\omega} G\left(h, \mathbb{D}^{\lambda}\right)\right|_{h} \leq B$. Let $\left(E, \mathbb{D}^{\lambda}, h\right)$ be analytically stable with respect to $\omega$. Then there exist constants $C_{i} \geq 0(i=1,2)$ with the following property:

- Let $s$ be any self-adjoint endomorphism satisfying $\|s\|_{P, h}<\infty, \operatorname{tr}(s)=0$ and $\sup \left|\Lambda_{\omega} G\left(h \cdot e^{s}, \mathbb{D}^{\lambda}\right)\right| \leq B$. Then the following inequality holds:

$$
\sup _{X}|s|_{h} \leq C_{1}+C_{2} \cdot M\left(h, h e^{s}\right)
$$

Sketch of the proof The excellent argument given in [17] works in the case of flat $\lambda$-connection without any essential change. Since we would like to use some minor variants of this proposition (Sections 2.4.5-2.4.6), we recall an outline of the proof for the convenience of readers. To begin with, we remark that we have only to show the following inequality for some $C_{i}^{\prime}>0$ due to Corollary 2.40 :

$$
\sup _{X}|s|_{h} \leq C_{1}^{\prime}+C_{2}^{\prime} \cdot \max \left\{0, M\left(h, h e^{s}\right)\right\}
$$

In the following, $C_{i}$ denote positive constants. As is noticed in Section 2.2.5, the inequality

$$
\Delta_{\omega}^{\lambda} \log \operatorname{tr}\left(e^{s}\right) \leq|\Lambda G(h)|_{h}+\left|\Lambda G\left(h e^{s}\right)\right|_{h e^{s}} \leq 2 B
$$

holds. Hence, there exist $C_{i}>0(i=3,4)$ such that the inequality

$$
\sup \log \operatorname{tr}\left(e^{s}\right) \leq C_{3}+C_{4} \cdot \int \log \operatorname{tr}\left(e^{s}\right)
$$

holds for any $s$ as above, due to Condition 2.33. Because

$$
C_{5}+C_{6} \cdot|s|_{h} \leq \log \operatorname{tr} e^{s} \leq C_{7}+C_{8} \cdot|s|_{h}
$$

for some $C_{i}>0(i=5,6,7,8)$, there exist $C_{i}(i=9,10)$ such that the following holds for any $s$ as above:

$$
\sup |s|_{h} \leq C_{9}+C_{10} \cdot \int|s|_{h}
$$

Assume that the claim of the proposition does not hold, and we will derive a contradiction. Under the assumption, either one of the following occurs:

Case 1 There exists a sequence $\left\{s_{i} \in \mathcal{P}\left(S_{h}\right) \mid i=1,2, \cdots\right\}$ such that $\sup \left|s_{i}\right|_{h} \longrightarrow \infty$ and $M\left(h, h e^{s_{i}}\right) \leq 0$. 
Case 2 There exist sequences $\left\{s_{i} \in \mathcal{P}\left(S_{h}\right)\right\}$ and $\left\{C_{2, i} \in \mathbf{R}\right\}$ with the following properties:

$$
\begin{gathered}
\sup _{X}\left|s_{i}\right| \longrightarrow \infty, \quad C_{2, i} \longrightarrow \infty, \quad(i \longrightarrow \infty) \\
M\left(h, h e^{s_{i}}\right)>0, \quad \sup \left|s_{i}\right|_{h} \geq C_{2, i} M\left(h, h e^{s_{i}}\right)
\end{gathered}
$$

In both the cases, we have $\left\|s_{i}\right\|_{L^{1}} \longrightarrow \infty$ due to (17). We put $\ell_{i}:=\left\|s_{i}\right\|_{L^{1}}$ and $u_{i}:=s_{i} / \ell_{i}$. Clearly, we have $\left\|u_{i}\right\|_{L^{1}}=1$ and uniform boundedness $\sup _{X}\left|u_{i}\right|<C$ due to (17). In the following, let $L^{2}\left(S_{h}\right)$ (resp. $L_{1}^{2}\left(S_{h}\right)$ ) denote the space of $L^{2}-$ sections (resp. $L_{1}^{2}$-sections) of $S_{h}$. The following lemma is one of the keys in the proof of Proposition 2.41 .

Lemma 2.42 After going to an appropriate subsequence, $\left\{u_{i}\right\}$ weakly converges to some $u_{\infty} \neq 0$ in $L_{1}^{2}\left(S_{h}\right)$. Moreover, we have the following inequality, for any $C^{\infty}$-function $\Phi: \mathbf{R} \times \mathbf{R} \longrightarrow \mathbf{R}_{\geq 0}$ such that $\Phi\left(y_{1}, y_{2}\right) \leq\left(y_{1}-y_{2}\right)^{-1}$ for $y_{1}>y_{2}$ :

$$
\sqrt{-1} \int \operatorname{tr}\left(u_{\infty} \Lambda_{\omega} G(h)\right)+\int_{X}\left(\Phi\left(u_{\infty}\right) \mathbb{D}^{\lambda} u_{\infty}, \mathbb{D}^{\lambda} u_{\infty}\right)_{h, \omega} \leq 0 .
$$

Proof By considering $\Phi-\epsilon$ for any small positive number $\epsilon$, we have only to consider the case $\Phi\left(y_{1}, y_{2}\right)<\left(y_{1}-y_{2}\right)^{-1}$ for $y_{1}>y_{2}$. In both the cases, we have the inequalities for some positive constant $C$, from the formula (15):

$$
\ell_{i} \sqrt{-1} \int_{X} \operatorname{tr}\left(u_{i} \Lambda_{\omega} G\left(h, \mathbb{D}^{\lambda}\right)\right)+\ell_{i}^{2} \int\left(\Psi\left(\ell_{i} u_{i}\right) \mathbb{D}^{\lambda} u_{i}, \mathbb{D}^{\lambda} u_{i}\right)_{h} \leq \ell_{i} \cdot \frac{C}{C_{2, i}} .
$$

(In the case 1 , we take any sequence $\left\{C_{2, i}\right\}$ such that $C_{2, i} \longrightarrow \infty$ ). Let $\Phi$ be as above. Due to the uniform boundedness of $u_{i}$, we may assume that $\Phi$ has the compact support. Then if $\ell$ is sufficiently large, we have $\Phi\left(\lambda_{1}, \lambda_{2}\right)<\ell \Psi\left(\ell \lambda_{1}, \ell \lambda_{2}\right)$. Therefore, we obtain the following inequality:

$$
\sqrt{-1} \int_{X} \operatorname{tr}\left(u_{i} \Lambda_{\omega} G\left(h, \mathbb{D}^{\lambda}\right)\right)+\int_{X}\left(\Phi\left(u_{i}\right) \mathbb{D}^{\lambda} u_{i}, \mathbb{D}^{\lambda} u_{i}\right)_{h, \omega} \leq \frac{C}{C_{2, i}} .
$$

Since $\sup _{X}\left|u_{i}\right|$ is bounded independently of $i$, there exists a function $\Phi$ as above which satisfies $\Phi\left(u_{i}\right)=c \cdot \mathrm{id}$, moreover, for some small positive number $c>0$. Therefore, we obtain the boundedness of $\left\{u_{i}\right\}$ in $L_{1}^{2}$. By taking an appropriate subsequence, $\left\{u_{i}\right\}$ is weakly convergent in $L_{1}^{2}$. Let $u_{\infty}$ denote the weak limit. Let $Z$ be any compact subset of $X$. Then $\left\{u_{i}\right\}$ is convergent to $u_{\infty}$ on $Z$ in $L^{2}$, and hence $\int_{Z}\left|u_{i}\right| \rightarrow \int_{Z}\left|u_{\infty}\right|$. Since $\sup \left|u_{i}\right|$ are uniformly bounded, we obtain $\int_{Z}\left|u_{\infty}\right| \neq 0$, if the volume of $X-Z$ is sufficiently small. Thus, $u_{\infty} \neq 0$. Similarly, we can show the convergence $\int \operatorname{tr}\left(u_{i} \Lambda G\left(h, \mathbb{D}^{\lambda}\right)\right) \longrightarrow \int \operatorname{tr}\left(u_{\infty} \Lambda G\left(h, \mathbb{D}^{\lambda}\right)\right)$. Since $\left\{u_{i}\right\}$ are 
weakly convergent to $u_{\infty}$ in $L_{1}^{2}$, we have the almost everywhere convergence of $\left\{u_{i}\right\}$ and $\left\{\mathbb{D}^{\lambda} u_{i}\right\}$ to $u_{\infty}$ and $\mathbb{D}^{\lambda} u_{\infty}$ respectively. Therefore, the sequence $\left\{\Phi\left(u_{i}\right) \mathbb{D}^{\lambda} u_{i}\right\}$ converges to $\Phi\left(u_{\infty}\right) \mathbb{D}^{\lambda} u_{\infty}$ almost everywhere. Hence, we have

$$
\int\left(\Phi\left(u_{\infty}\right) \mathbb{D}^{\lambda} u_{\infty}, \mathbb{D}^{\lambda} u_{\infty}\right)_{h, \omega} \leq \underline{\lim } \int\left(\Phi\left(u_{i}\right) \mathbb{D}^{\lambda} u_{i}, \mathbb{D}^{\lambda} u_{i}\right)_{h, \omega}
$$

due to Fatou's lemma. Thus, we obtain the desired inequality, and the proof of Lemma 2.42 is finished.

We reproduce the rest of the excellent argument given in [17] just for the completeness. We do not use it in the later argument. The point is that the existence of a nontrivial section $u_{\infty}$ as in Lemma 2.42 contradicts the analytic stability of $\left(E, \mathbb{D}^{\lambda}, h\right)$.

Lemma 2.43 The eigenvalues of $u_{\infty}$ are constant, and $u_{\infty}$ has at least two distinct eigenvalues.

Proof To show the constantness of the eigenvalues, we have only to show the constantness of $\operatorname{tr}\left(\varphi\left(u_{\infty}\right)\right)$ for any $C^{\infty}$-function $\varphi: \mathbf{R} \longrightarrow \mathbf{R}$. We have $(\bar{\partial}+\lambda \partial) \operatorname{tr} \varphi\left(u_{\infty}\right)=$ $\operatorname{tr}\left(\mathbb{D}^{\lambda} \varphi\left(u_{\infty}\right)\right)=\operatorname{tr}\left(d \varphi\left(u_{\infty}\right) \mathbb{D}^{\lambda} u_{\infty}\right)$. Let $N$ be any large number. We can take a $C^{\infty}$-function $\Phi: \mathbf{R} \times \mathbf{R} \longrightarrow \mathbf{R}$ such that $\Phi\left(y_{1}, y_{1}\right)=d \varphi\left(y_{1}, y_{1}\right)$ and $N \Phi^{2}\left(y_{1}, y_{2}\right)<$ $\left(y_{1}-y_{2}\right)^{-1}$ for $y_{1}>y_{2}$. We obtain $\operatorname{tr}\left(d \varphi\left(u_{\infty}\right)\left(\mathbb{D}^{\lambda} u_{\infty}\right)\right)=\operatorname{tr}\left(\Phi\left(u_{\infty}\right) \mathbb{D}^{\lambda} u_{\infty}\right)$ due to the first condition. We obtain the following inequality from Lemma 2.42:

$$
\int_{X}\left|\Phi\left(u_{\infty}\right) \mathbb{D}^{\lambda} u_{\infty}\right|^{2} \leq-\frac{\sqrt{-1}}{N} \int_{X} \operatorname{tr}\left(u_{\infty} \Lambda G(h)\right) .
$$

Therefore, $\left|(\bar{\partial}+\lambda \partial) \operatorname{tr} \varphi\left(u_{\infty}\right)\right|_{L^{2}}^{2}=0$. Thus, the eigenvalues of $u_{\infty}$ are constant. Since $\operatorname{tr}\left(u_{\infty}\right)=0$ and $u_{\infty} \neq 0, u_{\infty}$ has at least two distinct eigenvalues.

Let $\kappa_{1}<\kappa_{2}<\cdots<\kappa_{w}$ denote the constant distinct eigenvalues of $u_{\infty}$. Then $\varphi\left(u_{\infty}\right)$ and $\Phi\left(u_{\infty}\right)$ depend only on the values $\varphi\left(\kappa_{i}\right)$ and $\varphi\left(\kappa_{i}, \kappa_{j}\right)$ respectively.

Lemma 2.44 Let $\Phi: \mathbf{R}^{2} \longrightarrow \mathbf{R}$ be a $C^{\infty}$-function such that $\Phi\left(\kappa_{i}, \kappa_{j}\right)=0$ for $\kappa_{i}>\kappa_{j}$. Then $\Phi\left(u_{\infty}\right)\left(\mathbb{D}^{\lambda} u_{\infty}\right)=0$.

Proof We may replace $\Phi$ with $\Phi_{1}$ satisfying $\Phi_{1}\left(\kappa_{i}, \kappa_{j}\right)=0$ for $\kappa_{i}>\kappa_{j}$ and $N \Phi_{1}^{2}\left(y_{1}, y_{2}\right)<\left(y_{1}-y_{2}\right)^{-1}$ for $y_{1}>y_{2}$. Then we obtain $\left\|\Phi_{1}\left(u_{\infty}\right) \mathbb{D}^{\lambda} u_{\infty}\right\|_{L^{2}}^{2} \leq C / N$ due to Lemma 2.42, and hence we obtain $\Phi\left(u_{\infty}\right) \mathbb{D}^{\lambda} u_{\infty}=\Phi_{1}\left(u_{\infty}\right) \mathbb{D}^{\lambda} u_{\infty}=0$. 
Let $\gamma_{i}$ denote the open interval $] \kappa_{i}, \kappa_{i+1}\left[\right.$. Let $p_{\gamma}: \mathbf{R} \longrightarrow[0,1]$ be any decreasing $C^{\infty}$-function such that $p_{\gamma}\left(\kappa_{i}\right)=1$ and $p_{\gamma}\left(\kappa_{i+1}\right)=0$. We put $\pi_{\gamma}=p_{\gamma}\left(u_{\infty}\right)$. It is easy to see that $\pi_{\gamma}$ is $L_{1}^{2}$. Due to $p_{\gamma}^{2}=p_{\gamma}$, we have $\pi_{\gamma}^{2}=\pi_{\gamma}$. We have $\mathbb{D}^{\lambda} \pi_{\gamma}=d p\left(u_{\infty}\right) \mathbb{D}^{\lambda} u_{\infty}$. We put $\Phi_{\gamma}\left(y_{1}, y_{2}\right)=\left(1-p_{\gamma}\right)\left(y_{2}\right) \cdot d p_{\gamma}\left(y_{1}, y_{2}\right)$, and then we have $\left(1-\pi_{\gamma}\right) \circ \mathbb{D}^{\lambda} \pi_{\gamma}=\Phi_{\gamma}\left(u_{\infty}\right) \circ \mathbb{D}^{\lambda} u_{\infty}$. On the other hand, since we have $\Phi_{\gamma}\left(\kappa_{i}, \kappa_{j}\right)=0\left(\kappa_{i}>\kappa_{j}\right)$, we obtain $\Phi_{\gamma}\left(u_{\infty}\right) \mathbb{D}^{\lambda} u_{\infty}=0$ due to Lemma 2.44. Therefore, we obtain $\left(1-\pi_{\gamma}\right) \circ \mathbb{D}^{\lambda} \pi_{\gamma}=0$.

From $\left(1-\pi_{\gamma}\right) d^{\prime \prime} \pi_{\gamma}=0$, we obtain a saturated coherent subsheaf $V_{\gamma}$ such that $\pi_{\gamma}$ is the orthogonal projection on $V_{\gamma}$ due to a result of Uhlenbeck and Yau [22]. From $\left(1-\pi_{\gamma}\right) d^{\prime} \pi_{\gamma}=0$, the bundle $V_{\gamma}$ is $\mathbb{D}^{\lambda}$-invariant. Since we consider the case $\lambda \neq 0$, it is easy to see that $V_{\gamma}$ is indeed a subbundle of $E$. Namely, we obtain a $\lambda$-flat subbundle $\left(V_{\gamma}, \mathbb{D}_{V_{\gamma}}^{\lambda}\right) \subset\left(E, \mathbb{D}^{\lambda}\right)$.

Let us show $\operatorname{deg}_{\omega}\left(V_{\gamma}, h_{\gamma}\right) / \operatorname{rank} V_{\gamma} \geq \operatorname{deg}_{\omega}(E, h) / \operatorname{rank} E$ for some $\gamma$, which contradicts the stability assumption of $\left(E, \mathbb{D}^{\lambda}, h\right)$, where $h_{\gamma}:=h_{\mid V_{\gamma}}$. From Lemma 2.34, we have

$$
\operatorname{deg}\left(V_{\gamma}\right)=\frac{1}{2 \pi} \frac{1}{1+|\lambda|^{2}}\left(\sqrt{-1} \int \operatorname{tr}\left(\pi_{\gamma} G(h)\right)-\int\left|\mathbb{D}^{\lambda} \pi_{\gamma}\right|^{2}\right) .
$$

We have $u_{\infty}=\kappa_{w} \cdot \operatorname{id}_{E}-\sum|\gamma| \cdot \pi_{\gamma}$, where $|\gamma|$ denotes the length of $\gamma$. We put

$$
\begin{aligned}
W & :=\kappa_{w} \operatorname{deg}(E)-\sum|\gamma| \cdot \operatorname{deg}\left(V_{\gamma}\right) \\
& =\frac{1}{2 \pi} \frac{1}{1+|\lambda|^{2}}\left(\sqrt{-1} \int \operatorname{tr}\left(u_{\infty} \Lambda G(h)\right)+\int \sum|\gamma| \cdot\left|\mathbb{D}^{\lambda} \pi_{\gamma}\right|^{2}\right) .
\end{aligned}
$$

Since $\mathbb{D}^{\lambda} \pi_{\gamma}=d p_{\gamma}\left(u_{\infty}\right) \mathbb{D}^{\lambda} u_{\infty}$, we obtain the following:

$$
W=\frac{1}{2 \pi} \frac{1}{1+|\lambda|^{2}}\left(\int \sqrt{-1} \operatorname{tr}\left(u_{\infty} \Lambda G(h)\right)+\int\left(\sum|\gamma| d p_{\gamma}\left(u_{\infty}\right)^{2} \mathbb{D}^{\lambda} u_{\infty}, \mathbb{D}^{\lambda} u_{\infty}\right)\right)
$$

We can check $\sum|\gamma|\left(d p_{\gamma}\right)\left(\kappa_{i}, \kappa_{j}\right)^{2}=\left(\kappa_{i}-\kappa_{j}\right)^{-1}$ for $\kappa_{i}>\kappa_{j}$ by a direct argument. Therefore, we obtain $W \leq 0$, due to Lemma 2.42 . Namely we obtain

$$
\kappa_{w} \cdot \operatorname{deg} E \leq \sum|\gamma| \cdot \operatorname{deg}\left(V_{\gamma}\right)
$$

On the other hand, we have $0=\operatorname{tr}\left(u_{\infty}\right)=\kappa_{w} \cdot \operatorname{rank} E-\sum|\gamma| \cdot \operatorname{rank} V_{\gamma}$. Therefore, we obtain $\operatorname{deg}\left(V_{\gamma}\right) / \operatorname{rank} V_{\gamma} \geq \operatorname{deg}(E) / \operatorname{rank} E$ for at least one of $\gamma$, which contradicts the stability of $\left(E, \mathbb{D}^{\lambda}, h\right)$. Thus, the proof of Proposition 2.41 is finished. 
2.4.5 Variant 1 of Proposition 2.41 Let $C$ be a smooth connected projective curve, and $D$ be a simple divisor. Let $\left(E, \mathbb{D}^{\lambda}\right)$ be a $\lambda$-flat bundle on $C-D$. Let $0<\eta<1 / 2$. Let $\epsilon_{0}>0$ be a sufficiently smaller number than $\eta$. Let $\omega_{\epsilon}\left(0 \leq \epsilon<\epsilon_{0}\right)$ be a Kahler metric of $C-D$ with the following conditions:

- Let $P \in D$. Let $(U, z)$ be a holomorphic coordinate around $P$ such that $z(P)=0$. Then the following holds for some positive constants $C_{i}(i=1,2)$ :

$$
C_{1} \cdot \omega_{\epsilon} \leq \epsilon^{2}|z|^{2 \epsilon} \frac{d z \cdot d \bar{z}}{|z|^{2}}+\eta^{2}|z|^{2 \eta} \frac{d z \cdot d \bar{z}}{|z|^{2}} \leq C_{2} \cdot \omega_{\epsilon}
$$

- $\omega_{\epsilon} \longrightarrow \omega_{0}$ as $\epsilon \rightarrow 0$ in the $C^{\infty}$-sense locally on $C-D$.

Suppose that we are given hermitian metrics $h^{(\epsilon)}$ of $E$ with the following properties:

- $\left|\Lambda_{\omega_{\epsilon}} G\left(h^{(\epsilon)}, \mathbb{D}^{\lambda}\right)\right|_{h^{(\epsilon)}} \leq C_{1}$, where the constant $C_{1}$ is independent of $\epsilon$.

- $\left\{h^{(\epsilon)}\right\}$ converges to $h^{(0)}$ as $\epsilon \rightarrow 0$ in the $C^{\infty}$-sense locally on $C-D$.

- $\left(E, \mathbb{D}^{\lambda}, h^{(0)}\right)$ is analytic stable.

Lemma 2.45 Let $s^{(\epsilon)}$ be self-adjoint endomorphisms of $\left(E, h^{(\epsilon)}\right)$ satisfying $\operatorname{tr} s^{(\epsilon)}=0$ and the following properties:

- $\left\|s^{(\epsilon)}\right\|_{P, h^{(\epsilon)}, \omega_{\epsilon}}<\infty$. But we do not assume the uniform boundedness.

- $\left|\Lambda_{\omega_{\epsilon}} G\left(h^{(\epsilon)} e^{s^{(\epsilon)}}, \mathbb{D}^{\lambda}\right)\right|_{h^{(\epsilon)}} \leq C_{1}$. The constant $C_{1}$ is independent of $\epsilon$.

Then there exist constants $C_{i}>0(i=3,4)$, which are independent of $\epsilon$, with the following property:

$$
\sup \left|s^{(\epsilon)}\right|_{h^{(\epsilon)}} \leq C_{3}+C_{4} \cdot M\left(h^{(\epsilon)}, h^{(\epsilon)} e^{s^{(\epsilon)}}\right) .
$$

Sketch of a proof The argument is essentially the same as the proof of Proposition 2.41. We assume that the claim does not hold, and we will derive a contradiction. After going to an appropriate subsequence, either one of the following holds:

Case $1 M\left(h^{(\epsilon)}, h^{(\epsilon)} e^{s^{(\epsilon)}}\right) \leq 0$ and $\sup _{C-D}\left|S^{(\epsilon)}\right|_{h^{(\epsilon)}} \longrightarrow \infty$ as $\epsilon \rightarrow 0$.

Case $2 M\left(h^{(\epsilon)}, h^{(\epsilon)} e^{s^{(\epsilon)}}\right)>0, \sup \left|s^{(\epsilon)}\right| \geq C_{2}^{(\epsilon)} M\left(h^{(\epsilon)}, h^{(\epsilon)} e^{s^{(\epsilon)}}\right), \sup \left|s^{(\epsilon)}\right|_{h^{(\epsilon)}} \longrightarrow \infty$ and $C_{2}^{(\epsilon)} \longrightarrow \infty$ as $\epsilon \rightarrow 0$.

By using Lemma 2.47 (given below) and the argument given in the first part of Proposition 2.41, we can show that there exist positive constants $C_{i}(i=5,6)$, which are independent of $\epsilon$, with the following property:

$$
\sup _{C-D}\left|S^{(\epsilon)}\right|_{h^{(\epsilon)}} \leq C_{5}+C_{6} \cdot \int\left|S^{(\epsilon)}\right|_{h^{(\epsilon)}} \operatorname{dvol}_{\omega_{\epsilon}} .
$$


We put $\ell^{(\epsilon)}:=\left\|s^{(\epsilon)}\right\|_{L^{1}}$ and $u^{(\epsilon)}:=s^{(\epsilon)} / \ell^{(\epsilon)}$. The following lemma is the counterpart of Lemma 2.42.

Lemma 2.46 We have a nontrivial $L_{1}^{2}$-section $u_{\infty}$ of $S_{h^{(0)}}$ with the following property:

- The following inequality holds for any $C^{\infty}$-function $\Phi: \mathbf{R} \times \mathbf{R} \longrightarrow \mathbf{R}_{\geq 0}$ such that $\Phi\left(y_{1}, y_{2}\right) \leq\left(y_{1}-y_{2}\right)^{-1}$ for $y_{1}>y_{2}$ :

$$
\int_{C-D}\left(\sqrt{-1} \operatorname{tr}\left(u_{\infty} \Lambda_{\omega_{0}} G\left(h^{(0)}\right)\right)+\left(\Phi\left(u_{\infty}\right) \mathbb{D}^{\lambda} u_{\infty}, \mathbb{D}^{\lambda} u_{\infty}\right)_{h^{(0)}, \omega_{0}}\right) \operatorname{dvol}_{\omega_{0}} \leq 0
$$

Proof The argument is essentially the same as the proof of Lemma 2.42. We have the following for some positive constant $C_{5}$ :

$$
\int_{C-D}\left(\sqrt{-1} \operatorname{tr}\left(u^{(\epsilon)} \Lambda_{\omega_{\epsilon}} G\left(h^{(\epsilon)}\right)\right)+\left(\Phi\left(u^{(\epsilon)}\right) \mathbb{D}^{\lambda} u^{(\epsilon)}, \mathbb{D}^{\lambda} u^{(\epsilon)}\right)_{h^{(\epsilon)}, \omega_{\epsilon}}\right) \operatorname{dvol}_{\omega_{\epsilon}} \leq \frac{C_{5}}{C_{2}^{(\epsilon)}}
$$

From this, we obtain the following boundedness as in the proof of Lemma 2.42:

$$
\int_{C-D}\left|\mathbb{D}^{\lambda} u^{(\epsilon)}\right|_{h^{(\epsilon)}}^{2} \operatorname{dvol}_{\omega_{\epsilon}}<C_{10}
$$

Let us take a sequence of $C^{\infty}$-isometries $F_{\epsilon}:\left(E, h^{(\epsilon)}\right) \longrightarrow\left(E, h^{(0)}\right)$ which converges to the identity of $E$, in the $C^{\infty}$-sense locally on $C-D$. Remark that the sequence $\left\{F_{\epsilon}\left(\mathbb{D}^{\lambda}\right)\right\}$ converges to $\mathbb{D}^{\lambda}$ as $\epsilon \rightarrow 0$ in the $C^{\infty}$-sense locally on $C-D$. The sequence $\left\{F_{\epsilon}\left(u^{(\epsilon)}\right)\right\}$ is bounded on $L_{1}^{2}$ locally on $C-D$. By going to an appropriate subsequence, we may assume that the sequence $\left\{u^{(\epsilon)}\right\}$ is weakly convergent in $L_{1}^{2}$ locally on $C-D$, and hence it is convergent in $L^{2}$ on any compact subset $Z \subset C-D$. Let $u_{\infty}$ denote the weak limit. We have $\int_{Z}\left|u^{(\epsilon)}\right| \longrightarrow \int_{Z}\left|u_{\infty}\right|$. Hence $\int_{Z}\left|u_{\infty}\right| \neq 0$, when the volume of $C-Z \cup D$ is sufficiently small, due to the boundedness of $\left\{\sup \left|u^{(\epsilon)}\right| \mid \epsilon>0\right\}$. In particular, $u_{\infty} \neq 0$. Similarly, we obtain $\int_{C-D} \operatorname{tr}\left(u^{(\epsilon)} G\left(h^{(\epsilon)}\right)\right) \longrightarrow \int_{C-D} \operatorname{tr}\left(u_{\infty} G\left(h^{(0)}\right)\right)$. Since we can derive the almost everywhere convergence $\Phi\left(u^{(\epsilon)}\right) \mathbb{D}^{\lambda} u^{(\epsilon)} \longrightarrow \Phi\left(u_{\infty}\right) \mathbb{D}^{\lambda} u_{\infty}$ and $u^{(\epsilon)} \longrightarrow u_{\infty}$, we obtain $\int_{C-D}\left(\Phi\left(u_{\infty}\right) \mathbb{D}^{\lambda} u_{\infty}, \mathbb{D}^{\lambda} u_{\infty}\right) \leq \underline{\lim } \int_{C-D}\left(\Phi\left(u^{(\epsilon)}\right) \mathbb{D}^{\lambda} u^{(\epsilon)}, \mathbb{D}^{\lambda} u^{(\epsilon)}\right)$ due to Fatou's lemma. Thus, the proof of Lemma 2.46 is finished.

The rest of the proof of Lemma 2.45 is completely the same as the argument for Proposition 2.41.

We have used the following lemma in the proof. 
Lemma 2.47 For any positive number $B$, there exist positive constants $C_{i}(i=1,2)$ with the following property:

- Let $\epsilon$ be any positive number such that $\epsilon<1 / 2$. Let $f$ be any nonnegative bounded $C^{\infty}$-function on $C-D$ such that $\Delta_{\omega_{\epsilon}}(f) \leq B$. Then the inequality $\sup (f) \leq C_{1}+C_{2} \int f \cdot \operatorname{dvol}_{\omega_{\epsilon}}$ holds.

Proof Let $\left(U_{P}, z\right)$ be as above for $P \in D$, and $U_{P}^{*}:=U_{P}-\{z=0\}$. On $U_{P}^{*}$, the inequality $\Delta_{\omega_{\epsilon}}(f) \leq B$ is equivalent to the following:

$$
\Delta_{g_{0}}(f) \leq B \cdot\left(\epsilon^{2} \frac{|z|^{2 \epsilon}}{|z|^{2}}+\eta^{2} \frac{|z|^{2 \eta}}{|z|^{2}}\right) .
$$

Here, $g_{0}:=d z \cdot d \bar{z}$. Because of the boundedness of $f$, (18) holds on $U_{\boldsymbol{P}}$. (See the proof of Proposition 2.2 of [17].) Then we obtain the following inequality on $U_{P}$ :

$$
\Delta_{g_{0}}(f-B \cdot \phi) \leq 0, \quad \phi=|z|^{2 \epsilon}+|z|^{2 \eta} .
$$

For any point $Q \in \Delta(P, 1 / 2)$, we have the following:

$$
(f-B \cdot \phi)(Q) \leq \frac{4}{\pi} \int_{\Delta(Q, 1 / 2)}(f-B \cdot \phi) \cdot \operatorname{dvol}_{g_{0}} .
$$

Therefore, there exist some constants $C_{i}(i=3,4)$ which are independent of $\epsilon$, such that the following holds:

$$
f(Q) \leq C_{3}+C_{4} \int f \cdot \operatorname{dvol}_{\omega_{\epsilon}} .
$$

Thus, we obtain the upper bound of $f(Q)$, when $Q$ is close to a point of $D$. We can obtain such an estimate when $Q$ is far from $D$, similarly and more easily.

2.4.6 Variant 2 of Proposition 2.41 We will use another variant. Let $\pi: \mathcal{C} \longrightarrow \Delta$ be a holomorphic family of smooth projective curves. Let $\mathcal{D} \subset \mathcal{C}$ be a relative divisor. Let $\left(E, \mathbb{D}^{\lambda}\right)$ be a $\lambda$-flat bundle on $\mathcal{C}^{*}:=\mathcal{C}-\mathcal{D}$. We denote the fiber $\pi^{-1}(t)$ by $\mathcal{C}_{t}$ for $t \in \Delta$. We set $\mathcal{C}_{t}^{*}:=\mathcal{C}_{t} \backslash \mathcal{D}$. The restriction $\left(E, \mathbb{D}^{\lambda}\right)_{\mid \mathcal{C}_{t}^{*}}$ is denoted by $\left(E_{t}, \mathbb{D}_{t}^{\lambda}\right)$. Let $\omega$ be a metric of the relative tangent bundle of $\mathcal{C}^{*} / \Delta$ such that $\omega \sim \eta^{2}|z|^{2 \eta-2} d z \cdot d \bar{z}$ around $\mathcal{D}$. Here, $\eta$ denotes a small positive number, and $z$ is a holomorphic function such that $z^{-1}(0)=\mathcal{D}$ and $d z \neq 0$. The restriction of $\omega$ to $\mathcal{C}_{t}^{*}$ is denoted by $\omega_{t}$ for $t \in \Delta$. Let $h$ be a $C^{\infty}$-hermitian metric of $E$ such that $\left|\Lambda_{\omega_{t}} G\left(\mathbb{D}_{t}^{\lambda}, h_{t}\right)\right|_{h_{t}} \leq C_{1}$ for any $t \in \Delta$, where a constant $C_{1}$ is independent of $t$, and $h_{t}$ denotes the restriction of $h$ to $\mathcal{C}_{t}^{*}$. We assume that $\left(E_{t}, \mathbb{D}_{t}^{\lambda}, h_{t}\right)$ are analytic stable. The following lemma can be shown by an argument similar to the proof of Lemma 2.47 . 
Lemma 2.48 There exist positive constants $C_{i}(i=3,4)$, which are independent of $t$, with the following property.

- Let $s^{(t)}$ be an element of $\mathcal{P}_{h_{t}}\left(E_{t}\right)$ satisfying $\operatorname{tr} s^{(t)}=0,\left\|s^{(t)}\right\|_{h_{t}, P}<\infty$ and $\left|\Lambda_{\omega_{t}} G\left(\mathbb{D}_{t}^{\lambda}, h_{t} e^{s^{(t)}}\right)\right| \leq C_{1}$. Then the inequality $\sup \left|s^{(t)}\right| \leq C_{3}+C_{4}$. $M\left(h_{t}, h_{t} e^{s^{(t)}}\right)$ holds.

\subsection{Review of some results on Hermitian-Einstein metrics due to Simpson}

2.5.1 Existence theorem and a consequence Let $(X, \omega)$ be a Kahler manifold satisfying Condition 2.33 , and let $\left(E, \mathbb{D}^{\lambda}, h_{0}\right)$ be a metrized flat $\lambda$-connection satisfying (13).

Proposition 2.49 (Simpson) Assume that $\left(E, \mathbb{D}^{\lambda}, h_{0}\right)$ is analytically stable with respect to $\omega$. Then there exists a hermitian metric $h=h_{0} \cdot s$ satisfying the following conditions:

- $h$ and $h_{0}$ are mutually bounded.

- $\operatorname{det}(h)=\operatorname{det}\left(h_{0}\right)$.

- $\mathbb{D}^{\lambda}(s)$ is $L^{2}$ with respect to $h_{0}$ and $\omega$.

- It satisfies the Hermitian Einstein condition $\Lambda_{\omega} G(h)^{\perp}=0$, where $G(h)^{\perp}$ denotes the trace free part of $G(h)$.

- The following equalities hold:

$$
\begin{gathered}
\int_{Y} \operatorname{tr}\left(G(h)^{2}\right) \cdot \omega^{n-2}=\int_{Y} \operatorname{tr}\left(G\left(h_{0}\right)^{2}\right) \cdot \omega^{n-2} \\
\int_{Y} \operatorname{tr}\left(G(h)^{\perp 2}\right) \cdot \omega^{n-2}=\int_{Y} \operatorname{tr}\left(G\left(h_{0}\right)^{\perp 2}\right) \cdot \omega^{n-2}
\end{gathered}
$$

Proof We need only a minor modification of the proof of Theorem 1, Proposition 3.5 and Lemma 7.4 of [17]. Indeed, we have only to replace $D^{\prime \prime}, D^{\prime}$ and $F(h)$ with $\mathbb{D}^{\lambda}, \mathbb{D}^{\lambda \star}$ and $G(h)$, and to make some obvious modification of positive constant multiplications, as was mentioned by Simpson himself. (See page 754 of [18], for example. Remark that " $D^{c}$ " corresponds to our $-\mathbb{D}^{\lambda \star}$, and hence our $G(h)$ is slightly different from his.) The author recommends the reader to read a quite excellent argument in [17]. However, we will use a result related with the Donaldson functionals, which follows from the proof. Hence, we recall a brief outline of the proof of Proposition 2.49. We will use the notation in Section 2.4. 
Let $h_{0}$ be a metric for $\left(E, \mathbb{D}^{\lambda}\right)$ satisfying the finiteness (14). Let us consider the heat equation for the self adjoint endomorphisms $s_{t}$ with respect to $h_{0}$ :

$$
s_{t}^{-1} \frac{d s_{t}}{d t}=-\sqrt{-1} \Lambda_{\omega} G\left(h_{t}\right)^{\perp} .
$$

A detailed argument to solve (19) is given in Section 6 of [17]. Moreover, $\Lambda_{\omega} G\left(h_{t}\right)$ is shown to be uniformly bounded. We do not reproduce them here.

Then we would like to show the existence of an appropriate subsequence $t_{i} \rightarrow \infty$ such that $\left\{s_{t_{i}}\right\}$ converges to $s_{\infty}$ weakly in $L_{2}^{p}$ locally on $X$, and we would like to show that $h_{\infty}=h_{0} \cdot s_{\infty}$ gives the desired Hermitian-Einstein metric. For that purpose, Simpson used the Donaldson functional $M\left(h_{0}, h_{0} s_{t_{i}}\right)$. As reviewed in Proposition 2.41, he showed that there exist positive constants $C_{i}(i=1,2)$ such that the following holds:

$$
\sup \left|\log s_{t}\right| \leq C_{1}+C_{2} \cdot M\left(h_{0}, h_{0} s_{t}\right)
$$

He also showed [17, Lemma 7.1] that $M\left(h_{0}, h_{0} s_{t}\right)$ is $C^{1}$ with respect to $t$, and that the following formula holds:

$$
\frac{d}{d t} M\left(h_{0}, h_{0} s_{t}\right)=-\int_{X}\left|\Lambda_{\omega} G\left(h_{t}\right)^{\perp}\right|_{h_{t}, \omega}^{2} \leq 0
$$

Because $M\left(h_{0}, h_{0}\right)=0$ by definition, we obtain $M\left(h_{0}, h_{0} s_{t}\right) \leq 0$ from (21). Then we obtain the boundedness of $s_{t}$ from (20). For the solution of (19), we have $\operatorname{det}\left(s_{t}\right)=1$. Hence, the boundedness of $s_{t}^{-1}$ follows. We also obtain the existence of a subsequence $\left\{t_{i}^{\prime}\right\}$ such that $\left|\Lambda_{\omega} G\left(h_{t_{i}}^{\prime}\right)\right|_{L^{2}} \longrightarrow 0$.

From the uniform boundedness of $s_{t}$ and $\Lambda_{\omega} G\left(h_{t}\right)$, we obtain the lower bound of $M\left(h_{0}, h_{0} s_{t}\right)$. (See Corollary 2.40 in this paper, for example.) Moreover, we obtain the uniform bound of $\int_{X}\left|\mathbb{D}^{\lambda} u_{t}\right|_{h_{0}}^{2}$ due to the positivity of $\Psi$ given in (16), where $s_{t}=\exp \left(u_{t}\right)$. Due to the boundedness of $s_{t}$ and $s_{t}^{-1}$, we also obtain the boundedness of $\int_{X}\left|\mathbb{D}^{\lambda} s_{t}\right|_{h_{0}}^{2}$. Then we obtain the $L_{1}^{2}$ boundedness. Hence, we can take a subsequence $\left\{t_{i}^{\prime \prime}\right\}$ such that $s_{t_{i}^{\prime \prime}}$ converges to some $s_{\infty}$ weakly in $L_{1}^{2}$ locally on $X-D$. By using some more excellent additional argument given on page 895 of [17], it can be shown that the convergence is weakly $L_{2}^{p}$ locally on $X-D$, for any $p$. As a result, we obtain a Hermitian-Einstein metric. Thus, a sketch of the proof of Proposition 2.49 is finished.

By the above argument, we can derive the following lemma, which we would like to use in the subsequent argument (Section 4.5.2).

Lemma 2.50 Let $h_{0}$ be the hermitian metric satisfying (13). Let $h_{\mathrm{HE}}$ be the HermitianEinstein metric obtained in Proposition 2.49. Then we have $M\left(h_{0}, h_{\mathrm{HE}}\right) \leq 0$. 
Proof Recall that $h_{\mathrm{HE}}$ is obtained as the limit $h_{0} \cdot s_{\infty}$ of some sequence $\left\{h_{0} s_{t_{i}}\right\}$, and we have $M\left(h_{0}, h_{0} \cdot s_{t_{i}}\right) \leq 0$. We use the formula (15). Let $Z$ be any compact subset of $X$. The sequence $\left\{s_{t_{i}}\right\}$ converges to $s_{\infty}$ in $C^{0}$ on $Z$. The sequence $\left\{\Lambda_{\omega} G\left(h_{t_{i}}\right)\right\}$ converges to $\Lambda_{\omega} G\left(h_{\mathrm{HE}}\right)$ weakly in $L^{2}$ on $Z$. Therefore, we have the convergence:

$$
\lim _{t_{i} \rightarrow \infty} \int_{Z} \operatorname{tr}\left(u_{t_{i}} \cdot \Lambda_{\omega} G\left(h_{t_{i}}\right)\right) \operatorname{dvol}_{\omega}=\int_{Z} \operatorname{tr}\left(u_{\infty} \cdot \Lambda_{\omega} G\left(h_{\mathrm{HE}}\right)\right) \operatorname{dvol}_{\omega} .
$$

Here, $u_{t}$ are given by $\exp \left(u_{t}\right)=s_{t}$. Since $\sup _{X}\left|s_{t}\right|$ and $\sup _{X}\left|\Lambda G\left(h_{t}\right)\right|$ are bounded independently of $t$, we can easily obtain the convergence:

$$
\lim _{t_{i} \rightarrow \infty} \int_{X} \operatorname{tr}\left(u_{t_{i}} \cdot \Lambda_{\omega} G\left(h_{t_{i}}\right)\right) \operatorname{dvol}_{\omega}=\int_{X} \operatorname{tr}\left(u_{\infty} \cdot \Lambda_{\omega} G\left(h_{\mathrm{HE}}\right)\right) \mathrm{dvol} \omega .
$$

We have the $C^{0}$-convergence of the sequence $\left\{\mathbb{D}^{\lambda} u_{t_{i}}\right\}$ to $\mathbb{D}^{\lambda} u_{\infty}$. Hence, we have the following inequality due to Fatou's lemma:

$$
\int_{X}\left(\Psi\left(u_{\infty}\right) \mathbb{D}^{\lambda} u_{\infty}, \mathbb{D}^{\lambda} u_{\infty}\right) \operatorname{dvol}_{\omega} \leq \underline{\lim } \int_{X}\left(\Psi\left(u_{t_{i}}\right) \mathbb{D}^{\lambda} u_{t_{i}}, \mathbb{D}^{\lambda} u_{t_{i}}\right) \operatorname{dvol}_{\omega}
$$

Then we obtain the desired inequality.

2.5.2 Uniqueness The following proposition can be shown by an argument similar to the proof of Proposition 2.6 of [14] via the method in [17]. We state it for the reference in the subsequent argument.

Proposition 2.51 Let $(X, \omega)$ be a complete Kahler manifold satisfying Condition 2.33 , and $\left(E, \mathbb{D}^{\lambda}\right)$ be a $\lambda$-flat bundle on $X$. Let $h_{i}(i=1,2)$ be hermitian metrics of $E$ such that $\Lambda_{\omega} G\left(h_{i}\right)=0$. We assume that $h_{i}(i=1,2)$ are mutually bounded. Then the following holds:

- We have the decomposition of $\lambda$-flat bundles $\left(E, \mathbb{D}^{\lambda}\right)=\bigoplus\left(E_{a}, \mathbb{D}_{a}^{\lambda}\right)$ which is orthogonal with respect to both of $h_{i}(i=1,2)$.

- The restrictions of $h_{i}$ to $E_{a}$ are denoted by $h_{i, a}$. Then there exist positive numbers $b_{a}$ such that $h_{1, a}=b_{a} \cdot h_{2, a}$.

Proof Let $s$ be determined by $h_{2}=h_{1} \cdot s$. We can show $\mathbb{D}^{\lambda} s=0$ by the argument explained in the proof of Proposition 2.6 of [14]. Note we are considering the case $\lambda \neq 0$. Hence, the eigen decomposition of $s$ is $\mathbb{D}^{\lambda}$-flat, which gives the desired decomposition. 


\subsection{Regular filtered $\lambda$-flat bundles associated to tame harmonic bundles}

2.6.1 Tame pluri-harmonic metric Recall the tameness condition for pluri-harmonic metric. Let $X$ be a complex manifold with a simple normal crossing divisor $D$. Let $\left(E, \mathbb{D}^{\lambda}\right)$ be a $\lambda$-flat bundle on $X-D$. Let $h$ be a pluri-harmonic metric of $\left(E, \mathbb{D}^{\lambda}\right)$. Then we have the induced Higgs bundle $\left(E, \bar{\partial}_{h}, \theta_{h}\right)$. Let $P$ be any point of $X$, and let $\left(U_{P}, z_{1}, \ldots, z_{n}\right)$ be a holomorphic coordinate around $P$ such that $D \cap U_{P}=\bigcup_{i=1}^{l}\left\{z_{i}=0\right\}$. Then we have the expression:

$$
\theta=\sum_{i=1}^{l} f_{i} \cdot \frac{d z_{i}}{z_{i}}+\sum_{j=l+1}^{n} g_{j} \cdot d z_{j} .
$$

The pluri-harmonic metric $h$ is called tame, if the coefficients of the characteristic polynomials $\operatorname{det}\left(t-f_{i}\right)$ and $\operatorname{det}\left(t-g_{j}\right)$ are holomorphic on $U_{P}$ for any $P$. A $\lambda$-flat bundle with tame pluri-harmonic metric is called a tame harmonic bundle. Recall that the "curve test" for tameness is valid.

Proposition 2.52 [15, Corollary 8.7] A pluri-harmonic metric $h$ for $\left(E, \mathbb{D}^{\lambda}\right)$ is tame, if and only if $h_{\mid C}$ is tame for any closed curve $C \subset X$ transversal with the smooth part of $D$.

From a holomorphic vector bundle $E$ with a hermitian metric $h$, we obtain the filtered sheaf $\mathbf{E}_{*}(h):=\left({ }_{\mathbf{c}} E \mid \mathbf{c} \in \mathbf{R}^{S}\right)$ as explained in Section 3.5 of [14]. We recall the following proposition.

Proposition 2.53 [15, Theorem 8.58, Theorem 8.59 and Corollary 8.89] Suppose $\left(E, \mathbb{D}^{\lambda}, h\right)$ is a tame harmonic bundle on $X-D$. Then $\left(\mathbf{E}_{*}(h), \mathbb{D}^{\lambda}\right)$ is a regular filtered $\lambda$-flat bundle.

In this situation, we say that $h$ is a pluri-harmonic metric for $\left(\mathbf{E}_{*}(h), \mathbb{D}^{\lambda}\right)$. We also say that $h$ is a pluri-harmonic metric for $\left(\mathbf{c} E(h)_{*}, \mathbb{D}^{\lambda}\right)$.

2.6.2 One dimensional case In the one dimensional case, Simpson established the Kobayashi-Hitchin correspondence for parabolic flat bundles and the parabolic Higgs bundles, ie, $\lambda$-flat bundles in the cases $\lambda=0,1$. His result can be generalized for any $\lambda$.

Proposition 2.54 (Simpson [18]) Let $X$ be a smooth irreducible projective curve, and $D$ be a simple divisor of $X$. Let $\left(\mathbf{E}_{*}, \mathbb{D}^{\lambda}\right)$ be a regular filtered $\lambda$-flat bundle on $(X, D)$. We put $E={ }_{\mathbf{c}} E_{\mid X-D}$. The following conditions are equivalent: 
- $\left(\mathbf{E}_{*}, \mathbb{D}^{\lambda}\right)$ is poly-stable with par-deg $\left(\mathbf{E}_{*}\right)=0$.

- There exists a harmonic metric $h$ of $\left(E, \mathbb{D}^{\lambda}\right)$, which is adapted to the parabolic structure of $\mathbf{E}_{*}$, ie, $\mathbf{E}_{*} \simeq \mathbf{E}_{*}(h)$.

Moreover, such a metric is unique up to obvious ambiguity. Namely, let $h_{i}(i=1,2)$ be two harmonic metrics as above. Then we have the decomposition of Higgs bundles $\left(E, \mathbb{D}^{\lambda}\right)=\bigoplus\left(E_{a}, \mathbb{D}_{a}^{\lambda}\right)$ satisfying the following:

- The decomposition is orthogonal with respect to both of $h_{i}$.

- The restrictions of $h_{i}$ to $E_{a}$ are denoted by $h_{i, a}$. Then there exist positive numbers $b_{a}$ such that $h_{1, a}=b_{a} \cdot h_{2, a}$.

2.6.3 Projective case Let $X$ be a smooth irreducible projective variety with an ample line bundle $L$, and let $D$ be a simple normal crossing hypersurface of $X$ with the irreducible decomposition $D=\bigcup_{i \in S} D_{i}$. Let $\left(E, \mathbb{D}^{\lambda}, h\right)$ be a tame harmonic bundle on $X-D$.

Proposition 2.55 Let $\left(\mathbf{E}_{*}, \mathbb{D}^{\lambda}\right)$ be the regular filtered $\lambda$-flat bundle on $(X, D)$ associated to $\left(E, \mathbb{D}^{\lambda}, h\right)$.

- $\left(\mathbf{E}_{*}, \mathbb{D}^{\lambda}\right)$ is $\mu_{L}$-polystable with par-deg ${ }_{L}\left(\mathbf{E}_{*}\right)=0$.

- Let $\left(\mathbf{E}_{*}, \mathbb{D}^{\lambda}\right)=\bigoplus_{j}\left(\mathbf{E}_{j *}, \mathbb{D}_{j}^{\lambda}\right) \otimes \mathbf{C}^{p(j)}$ be the canonical decomposition of $\mu_{L}-$ polystable regular filtered $\lambda$-flat bundle. Then we have the corresponding decomposition of the metric $h=\bigoplus h_{i} \otimes g_{i}$, where $h_{i}$ denote pluri-harmonic metrics of $\left(E_{i}, \mathbb{D}_{i}^{\lambda}\right)$ adapted to the parabolic structure, and $g_{i}$ denote metrics of $\mathbf{C}^{p(i)}$.

- We have the vanishings of characteristic numbers:

$$
\int_{X} \operatorname{par}^{-\mathrm{ch}_{2, L}}\left(\mathbf{E}_{*}\right)=\int_{X} \operatorname{par}^{-c_{1, L}^{2}}\left(\mathbf{E}_{*}\right)=0 .
$$

Proof The first two claims can be shown by the argument in the proof of Proposition 5.1 of [14]. The third claim can be shown by an argument similar to the proof of Proposition 5.3 of [14], which we explain briefly. We have only to consider the case $\operatorname{dim} X=2$. Since $h$ is pluri-harmonic, we have the following equalities due to Lemma 2.31 and Lemma 2.32 on $X-D$ :

$$
\begin{aligned}
\operatorname{tr} R\left(d^{\prime \prime}, h\right) & =\left(1+|\lambda|^{2}\right)^{-1} \operatorname{tr} G\left(h, \mathbb{D}^{\lambda}\right)=0 \\
\operatorname{tr}\left(R\left(d^{\prime \prime}, h\right)^{2}\right) & =\left(1+|\lambda|^{2}\right)^{-2} \cdot \operatorname{tr}\left(G\left(h, \mathbb{D}^{\lambda}\right)^{2}\right)=0
\end{aligned}
$$

We also have the norm estimate for the holomorphic sections of $\mathbf{c} E$. (It is explained in Section 2.5 of [14] for $\lambda=0$. Similar claims hold for any $\lambda$, as shown in Section 13.3 of [15].) Then the argument in the proof of Proposition 5.3 works. 
Proposition 2.56 Let $\left(\mathbf{E}_{*}, \mathbb{D}^{\lambda}\right)$ be a regular filtered $\lambda$-flat bundle on $(X, D)$. We put $\left(E, \mathbb{D}^{\lambda}\right):=\left(\mathbf{E}_{*}, \mathbb{D}^{\lambda}\right)_{\mid X-D}$. Let $h_{a}(a=1,2)$ be pluri-harmonic metrics of $\left(E, \mathbb{D}^{\lambda}\right)$ on $X-D$ which is adapted to the parabolic structure. Then we have the decomposition $\left(E, \mathbb{D}^{\lambda}\right)=\bigoplus\left(E_{i}, \mathbb{D}^{\lambda}\right)$ with the following properties:

- The decomposition is orthogonal with respect to both of $h_{a}(a=1,2)$. Hence, we have the decompositions $h_{a}=\bigoplus_{i} h_{a, i}$.

- There exist positive numbers $b_{i}$ such that $h_{1, i}=b_{i} \cdot h_{2, i}$.

The decomposition on $X-D$ is prolonged to $\left(\mathbf{E}_{*}, \mathbb{D}^{\lambda}\right)=\bigoplus\left(\mathbf{E}_{i *}, \mathbb{D}^{\lambda}\right)$ on $X$.

Proof Similar to Proposition 5.2 of [14].

\subsection{Some integral for nonflat $\lambda$-connection on a curve}

This subsection is preliminary for Corollary 3.16. Let $Y$ be a smooth projective curve, and let $D$ be a divisor. Let $(E, \mathbf{F})$ be a parabolic bundle on $(Y, D)$. Let $\mathbb{D}^{\lambda}$ be a $C^{\infty}$ $\lambda$-connection on $E_{\mid Y-D}$. In this subsection, we do not assume $\mathbb{D}^{\lambda}$ is flat, ie, $\left(\mathbb{D}^{\lambda}\right)^{2}$ may not be 0 . However, we assume that it is flat around an appropriate neighbourhood $U_{P}$ of each $P \in D$, and that $\left(E, \mathbf{F}, \mathbb{D}^{\lambda}\right)_{\mid U_{P}}$ is a parabolic $\lambda$-flat bundle. In particular, we have $\operatorname{Res}_{P}\left(\mathbb{D}^{\lambda}\right) \in \operatorname{End}\left(E_{\mid P}\right)$. We assume moreover that it is graded semisimple, for simplicity, ie, the induced endomorphism on $\mathrm{Gr}^{F}\left(E_{\mid P}\right)$ is semisimple for each $P \in D$. (By using an $\epsilon$-perturbation in Section 2.1.6, we can drop this assumption.)

For each $P \in D$, we have the generalized eigen decomposition $E_{\mid P}:=\bigoplus^{P} \mathbb{E}_{\alpha}$ of $\operatorname{Res}_{P}\left(\mathbb{D}^{\lambda}\right)$. We also have the filtration ${ }^{P} F$ of $E_{\mid P}$. Let us take a holomorphic frame $\mathbf{v}$ of $E_{\mid U_{P}}$, which is compatible with $\left({ }^{P} \mathbb{E},{ }^{P} F\right)$. We put $\alpha\left(v_{i}\right):=\operatorname{deg}^{\mathbb{E}}\left(v_{i}\right)$ and $a\left(v_{i}\right):=\operatorname{deg}^{F}\left(v_{i}\right)$. Let $h$ be a $C^{\infty}$-metric of $E_{\mid Y-D}$ such that $h\left(v_{i}, v_{j}\right)=|z|^{-2 a\left(v_{i}\right)}$ $(i=j)$ and $0(i \neq j)$. Let us decompose $\mathbb{D}^{\lambda}=d^{\prime \prime}+d^{\prime}$. Let us take a $(1,0)$-operator $d_{0}^{\prime}$ such that $d^{\prime \prime}+d_{0}^{\prime}$ is $C^{\infty} \lambda$-connection of $E$ on $Y$, not only on $Y-D$. We also assume $d_{0}^{\prime} \mathbf{v}=0$. We put $A:=d^{\prime}-d_{0}^{\prime}$, which is a $C^{\infty}$-section of $\operatorname{End}(E) \otimes$ $\Omega^{1,0}(\log D)$ on $Y$, and holomorphic around $D$. We have $\operatorname{tr} \operatorname{Res}_{P}(A)=\operatorname{tr} \operatorname{Res}_{P}\left(\mathbb{D}^{\lambda}\right)$.

Let $h_{0}$ be a $C^{\infty}$-metric of $E$ on $Y$ such that $h_{0}\left(v_{i}, v_{j}\right)$ is $1(i=j)$ or $0(i \neq j)$ on $U_{P}(P \in D)$. Let $s$ be the endomorphism determined by $h=h_{0} \cdot s$. Then $s$ is described by the diagonal matrix $\operatorname{diag}\left(|z|^{-2 a\left(v_{1}\right)}, \ldots,|z|^{-2 a\left(v_{r}\right)}\right)$ with respect to the frame $\mathbf{v}$ on $U_{P}$.

Although $\mathbb{D}^{\lambda}$ is not necessarily flat, we obtain the operators $\delta_{h}^{\prime}, \delta_{h}^{\prime \prime}, \bar{\partial}_{h}, \partial_{h}, \theta_{h}$ and $\theta_{h}^{\dagger}$ as in Section 2.2.1. We put wt $(E, \mathbf{F}, P):=\sum_{a \in \mathcal{P a r}(E, \mathbf{F}, P)} a \cdot \operatorname{rank}\left({ }^{P} \mathrm{Gr}_{a}^{F}(E)\right)$. 
Lemma 2.57 We have the following formula:

$$
\frac{\sqrt{-1}}{2 \pi} \int_{Y} \bar{\partial} \operatorname{tr} \theta=\frac{\lambda}{1+|\lambda|^{2}} \sum_{P}\left(\lambda^{-1} \cdot \operatorname{tr} \operatorname{Res}_{P} \mathbb{D}^{\lambda}+\operatorname{wt}(E, \mathbf{F}, P)\right)
$$

Proof Let $\delta_{h_{0}}^{\prime}$ denote the $(1,0)$-operator obtained from $d^{\prime \prime}$ and $h_{0}$ as in Section 2.2.1. Then we have

$$
\theta_{h}=\frac{1}{1+|\lambda|^{2}}\left(d^{\prime}-\lambda \cdot \delta_{h}^{\prime}\right)=\frac{1}{1+|\lambda|^{2}}\left(d_{0}^{\prime}-\lambda \cdot \delta_{h_{0}}^{\prime}\right)+\frac{1}{1+|\lambda|^{2}}\left(A-\lambda \cdot s^{-1} \delta_{h_{0}}^{\prime} s\right) .
$$

We would like to apply Stokes' formula to the integral of $\bar{\partial} \operatorname{tr} \theta_{h}$. If we do so, $d_{0}^{\prime}-\lambda \delta_{h_{0}}^{\prime}$ does not contribute, because it is the $C^{\infty}$-section of $\operatorname{End}(E) \otimes \Omega^{1,0}$. We have

$$
\frac{\sqrt{-1}}{2 \pi} \int_{Y} \bar{\partial} \operatorname{tr}(A)=\sum_{P} \operatorname{tr} \operatorname{Res}_{P} \mathbb{D}^{\lambda} .
$$

Since $s^{-1} \delta_{h_{0}}^{\prime} s$ is described by $\operatorname{diag}\left(-a\left(v_{1}\right), \ldots,-a\left(v_{r}\right)\right) \cdot d z / z$ with respect to $\mathbf{v}$ on $U_{P}(P \in D)$, we have

$$
\frac{\sqrt{-1}}{2 \pi} \int_{Y} \bar{\partial} \operatorname{tr}\left(s^{-1} \delta_{h_{0}}^{\prime} s\right)=\sum_{P} \sum_{i=1}^{\operatorname{rank} E}-a\left(v_{i}\right)=-\sum_{P} \operatorname{wt}(E, \mathbf{F}, P) .
$$

Therefore, we obtain the following formula:

$$
\frac{\sqrt{-1}}{2 \pi} \frac{1+|\lambda|^{2}}{\lambda} \int \bar{\partial} \operatorname{tr} \theta_{h}=\sum_{P}\left(\lambda^{-1} \operatorname{tr} \operatorname{Res}_{P} \mathbb{D}^{\lambda}+\operatorname{wt}(E, \mathbf{F}, P)\right)
$$

Thus, we obtain (22).

\section{Ordinary metric and some consequences}

In this section, we mainly study graded semisimple regular filtered $\lambda$-flat bundles satisfying the SPW-condition (Definition 2.6). We will construct an ordinary metric for such a regular filtered $\lambda$-flat bundle in Section 3.3. We give an estimate of the induced operators by using the preliminary results in Sections 3.1-3.2, Then we show the existence of Hermitian-Einstein metric for such a parabolic $\lambda$-flat bundle, if it is $\mu_{L}$-stable (Proposition 3.19).

Some results in this section are available for any regular filtered $\lambda$-flat bundles which do not necessarily satisfy graded semisimplicity and the SPW-condition. One is Bogomolov-Gieseker inequality (Corollary 3.20). The others are formulas to express 
parabolic characteristic numbers in terms of the data at $D$ as in Section 3.5. The method of $\epsilon$-perturbation (Section 2.1.6) is used to deduce them from the results in the case that graded semisimplicity and the SPW-condition are satisfied.

\subsection{Around the intersection of the divisor}

3.1.1 Some estimates We put $X:=\Delta_{z}^{2}, D_{i}:=\left\{z_{i}=0\right\}$ and $D:=D_{1} \cup D_{2}$. Let $\left(\mathbf{E}_{*}, \mathbb{D}^{\lambda}\right)$ be a graded semisimple regular filtered $\lambda$-flat bundle on $(X, D)$, satisfying the SPW-condition. We take $c_{i} \notin \mathcal{P} \operatorname{ar}\left(\mathbf{E}_{*}, \mathbb{D}^{\lambda}\right)$ for $i=1,2$. By the assumption, there exist a positive integer $m$ and real numbers $\gamma_{i}$ with $-1 / m<\gamma_{i} \leq 0$, such that

$$
\mathcal{P a r}\left({ }_{\mathbf{c}} E_{*}, i\right) \subset\left\{c_{i}+\gamma_{i}+p / m \mid p \in \mathbf{Z},-1<\gamma_{i}+p / m<0\right\} .
$$

We put $\tilde{X}:=\Delta_{\zeta}^{2}, \widetilde{D}_{i}:=\left\{\zeta_{i}=0\right\}$ and $\widetilde{D}=\widetilde{D}_{1} \cup \widetilde{D}_{2}$. Let $\varphi: \tilde{X} \longrightarrow X$ be the ramified covering given by $\varphi\left(\zeta_{1}, \zeta_{2}\right)=\left(\zeta_{1}^{m}, \zeta_{2}^{m}\right)$. Let $\operatorname{Gal}(\tilde{X} / X)$ denote the Galois group of $\tilde{X} / X$. Recall the construction in [9]. For any $\mathbf{a} \in \mathbf{R}^{2}$, let ${ }_{\mathbf{a}} \widetilde{E}$ denote the subsheaf of $\widetilde{\mathbf{E}}:=\varphi^{*}(\mathbf{E})$ given as follows:

$$
{ }_{\mathbf{a}} \widetilde{E}:=\sum_{\mathbf{n}+m \mathbf{d} \leq \mathbf{a}} \varphi^{*}\left({ }_{\mathbf{d}} E\right) \cdot \prod_{i=1,2} \zeta_{i}^{-n_{i}}
$$

Then it is easy to see that $\widetilde{\mathbf{E}}_{*}=\left({ }_{\mathbf{a}} \widetilde{E} \mid \mathbf{a} \in \mathbf{R}^{2}\right)$ is a filtered bundle, and the induced flat $\lambda$-connection $\widetilde{\mathbb{D}}^{\lambda}$ is regular. We put $\widetilde{c}_{i}:=m \cdot\left(\gamma_{i}+c_{i}\right)$. By the assumption, $\operatorname{Par}\left(\widetilde{\mathbf{E}}_{*}, i\right)=\left\{p+\widetilde{c}_{i} \mid p \in \mathbf{Z}\right\}$.

We have the generalized eigen decompositions ${ }_{\mathrm{c}} E_{\mid D_{i}}=\bigoplus^{i} \mathbb{E}_{\alpha}$ with respect to the residue $\operatorname{Res}_{i}\left(\mathbb{D}^{\lambda}\right)$. We have the parabolic filtration ${ }^{i} F$ of $\mathbf{c} E$. Let $\mathbf{v}$ be a frame of $\mathbf{c} E$ compatible with ${ }^{i} F$ and ${ }^{i} \mathbb{E}(i=1,2)$. We put

$$
a_{i}\left(v_{j}\right):={ }^{i} \operatorname{deg}^{F}\left(v_{j}\right)-\left(c_{i}+\gamma_{i}\right) \in \frac{1}{m} \cdot \mathbf{Z}_{\leq 0} .
$$

Let $\alpha_{i}\left(v_{j}\right) \in \mathbf{C}$ denote the complex numbers determined by $v_{j \mid D_{i}} \in{ }^{i} \mathbb{E}_{\alpha_{i}\left(v_{j}\right)}$. We put

$$
\tilde{v}_{j}:=\varphi^{*}\left(v_{j}\right) \cdot \prod_{i=1,2} \zeta_{i}^{m a_{i}\left(v_{j}\right)}
$$

Then $\widetilde{\mathbf{v}}:=\left(\widetilde{v}_{j}\right)$ gives the frame of $\tilde{\mathbf{c}} \widetilde{E}$. We put $\beta_{i}\left(v_{j}\right):=m\left(\lambda \cdot a_{i}\left(v_{j}\right)+\alpha_{i}\left(v_{j}\right)\right)$. Let $\Gamma$ be the diagonal matrix whose $(j, j)$-entries are $\sum_{i=1,2} \beta_{i}\left(v_{j}\right) \cdot d \zeta_{i} / \zeta_{i}$. Let $A$ be determined by $\widetilde{\mathbb{D}}^{\lambda} \widetilde{\mathbf{v}}=\widetilde{\mathbf{v}} \cdot A$, and let $A_{0}:=A-\Gamma$. In the following, let $F_{\Gamma} \in$ $\operatorname{End}(\tilde{\mathbf{c}} \widetilde{E}) \otimes \Omega^{1}(\log \widetilde{D})$ be determined by $F_{\Gamma}(\widetilde{\mathbf{v}})=\widetilde{\mathbf{v}} \cdot \Gamma$. We put $\widetilde{\mathbb{D}}_{0}^{\lambda}:=\widetilde{\mathbb{D}}^{\lambda}-F_{\Gamma}$.

We have the expression $A_{0}=\sum_{i=1,2} A_{0}^{i} \cdot d \zeta_{i}$. If $m$ is sufficiently large, we may assume the following: 
(A) $A_{0}^{i}=O\left(\zeta_{i}^{2}\right)$. Moreover, $\left(A_{0}^{1}\right)_{j, k}=O\left(\zeta_{1}^{2} \cdot \zeta_{2}^{2}\right)$ in the case $\beta_{2}\left(v_{j}\right) \neq \beta_{2}\left(v_{k}\right)$, and $\left(A_{0}^{2}\right)_{j, k}=O\left(\zeta_{1}^{2} \cdot \zeta_{2}^{2}\right)$ in the case $\beta_{1}\left(v_{j}\right) \neq \beta_{1}\left(v_{k}\right)$.

Let $\tilde{h}$ be the hermitian metric of $\widetilde{\mathbf{c}} \widetilde{E}$ determined by $\tilde{h}\left(\widetilde{v}_{i}, \widetilde{v}_{j}\right)=\delta_{i, j} \cdot\left|\zeta_{1}\right|^{-2 \widetilde{c}_{1}} \cdot\left|\zeta_{2}\right|^{-2 \widetilde{c}_{2}}$. Let $\tilde{\theta}$ (resp. $\tilde{\theta}_{0}$ ) be the section of $\operatorname{End}(\tilde{E}) \otimes \Omega^{1}$ on $\tilde{X}-\tilde{D}$ induced by $\tilde{h}$ and $\widetilde{\mathbb{D}}^{\lambda}$ (resp. $\widetilde{\mathbb{D}}_{0}^{\lambda}$ ) as in Section 2.2.1. Let $\tilde{\theta}^{\dagger}$ and $\widetilde{\theta}_{0}^{\dagger}$ denote the adjoint of $\widetilde{\theta}$ and $\tilde{\theta}_{0}$, respectively. Denote the Euclidean metric of $\tilde{X}$ by $\widetilde{g}$.

\section{Lemma 3.1}

- $\left[\widetilde{\theta}, \widetilde{\theta}^{\dagger}\right]$ is bounded with respect to $\tilde{h}$ and $\tilde{g}$.

- $\tilde{\theta}^{2}=O\left(z_{1} \cdot z_{2}\right) \cdot d z_{1} \cdot d z_{2}$.

Proof We have the relations $\tilde{\theta}=\tilde{\theta}_{0}+\left(1+|\lambda|^{2}\right)^{-1} F_{\Gamma}$ and $\tilde{\theta}^{\dagger}=\tilde{\theta}_{0}^{\dagger}+\left(1+|\lambda|^{2}\right)^{-1} F_{\Gamma}^{\dagger}$. Hence, we have the following:

$$
\left[\tilde{\theta}, \tilde{\theta}^{\dagger}\right]=\left[\tilde{\theta}_{0}, \tilde{\theta}_{0}^{\dagger}\right]+\left(1+|\lambda|^{2}\right)^{-1}\left[\tilde{\theta}_{0}, F_{\Gamma}^{\dagger}\right]+\left(1+|\lambda|^{2}\right)^{-1}\left[\tilde{\theta}_{0}^{\dagger}, F_{\Gamma}\right]
$$

We have the decomposition of $\tilde{\theta}_{0}$ into the sum

$$
\lambda\left(1+|\lambda|^{2}\right)^{-1} \sum \tilde{c}_{i} \cdot d \zeta_{i} / \zeta_{i}+\tilde{\theta}_{0}^{\prime},
$$

where $\tilde{\theta}_{0}^{\prime}$ is the $C^{\infty}$-section of $\operatorname{End}(\tilde{\mathbf{c}} \tilde{E}) \otimes \Omega_{\tilde{X}}^{1}$ on $\tilde{X}$. Hence, $\left[\tilde{\theta}_{0}, \tilde{\theta}_{0}^{\dagger}\right]$ is the $C^{\infty}$ section of $\operatorname{End}(\tilde{\mathbf{c}} \tilde{E}) \otimes \Omega^{2}$ on $\tilde{X}$. Note the following:

$$
\tilde{\theta}_{0}^{\prime} \tilde{\mathbf{v}}=\tilde{\mathbf{v}} \cdot \frac{1}{1+|\lambda|^{2}} A_{0}
$$

(See Section 2.2.2, for example.) By Condition (A), $\left[\tilde{\theta}_{0}, F_{\Gamma}^{\dagger}\right]$ and $\left[F_{\Gamma}, \tilde{\theta}_{0}^{\dagger}\right]$ is also bounded. We have $\widetilde{\theta}^{2}=\widetilde{\theta}_{0}^{2}+2\left[\tilde{\theta}_{0}, F_{\Gamma}\right]$. Then we obtain the desired estimate for $\widetilde{\theta}^{2}$ by Condition (A).

Lemma 3.2 We have the boundedness of $G\left(\widetilde{\mathbb{D}}^{\lambda}, \widetilde{h}\right)$ and $\widetilde{\theta}^{2} \cdot \widetilde{\theta}^{\dagger}$ with respect to $\tilde{h}$ and $\tilde{g}$.

Proof The boundedness of $\tilde{\theta}^{2} \cdot \tilde{\theta}^{\dagger}$ follows from the estimate for $\tilde{\theta}^{2}$. We have the following equality:

$$
G\left(\widetilde{\mathbb{D}}^{\lambda}, \tilde{h}\right)=\left(1+|\lambda|^{2}\right) \cdot R\left(\tilde{h}, d^{\prime \prime}\right)-\frac{\left(1+|\lambda|^{2}\right)^{2}}{\lambda}\left(\bar{\partial}_{\tilde{h}}^{2}+\widetilde{\theta}^{2}-\lambda\left[\tilde{\theta}, \tilde{\theta}^{\dagger}\right]\right)
$$

(See Section 2.2.4.) We have the vanishing of the curvature $R\left(\tilde{h}, d^{\prime \prime}\right)=0$, and the relation $\lambda^{-1} \bar{\partial}_{\widetilde{h}}^{2}=\bar{\lambda}^{-1}\left(\widetilde{\theta}^{\dagger}\right)^{2}$. Hence, we obtain the boundedness of $G\left(\widetilde{\mathbb{D}}^{\lambda}, \widetilde{h}\right)$ from Lemma 3.1. 
Since $\tilde{h}$ is $\operatorname{Gal}(\tilde{X} / X)$-equivariant, we obtain the induced metric $h$ of $E$ on $X-D$. Clearly, $h$ is given by $h\left(v_{i}, v_{j}\right)=\delta_{i, j} \cdot\left|z_{1}\right|^{-2 a\left(v_{i}\right)} \cdot\left|z_{2}\right|^{-2 a\left(v_{j}\right)}$. Let $\theta$ be the section of $\operatorname{End}(E) \otimes \Omega^{1,0}$ on $X-D$ induced by $\mathbb{D}^{\lambda}$ and $h$, and let $\theta^{\dagger}$ denote the adjoint of $\theta$. Let $g_{m}$ denote the metric of $X-D$ given by $g_{m}=\sum\left|z_{i}\right|^{2(-1+1 / m)} \cdot d z_{i} \cdot d \bar{z}_{i}$.

Corollary 3.3 We have the boundedness of $G\left(\mathbb{D}^{\lambda}, h\right)$ and $\theta^{2} \cdot \theta^{\dagger}$ with respect to $g_{m}$ and $h$.

3.1.2 The induced metric and the $\lambda$-connection on the divisors For simplicity, we assume $c_{i}=\gamma_{i}=0(i=1,2)$ in this subsection. Let $(a, \alpha) \in \mathcal{K} \mathcal{M S}\left({ }^{\diamond} E, i\right)$. Let $\rho$ be a $C^{\infty}$-function on $X$ such that $\rho>0$. We put $\chi:=\rho \cdot\left|z_{1}\right|^{2}$. Let $D_{i}^{\circ}:=D_{i}-\left(D_{1} \cap D_{2}\right)$. We study the induced hermitian metric and the induced $\lambda$-connection of ${ }^{i} \mathrm{Gr}_{a, \alpha}^{F, \mathbb{E}}\left({ }^{\diamond} E\right)_{\mid D_{i}^{\circ}}$ $(i=1,2)$, depending on the choice of $\rho$. Let us consider the case $i=1$. Let $u_{j}$ $(j=1,2)$ be sections of ${ }^{1} \operatorname{Gr}_{a, \alpha}^{F, \mathbb{E}}\left({ }^{\diamond} E\right)$. We take sections $u_{j}^{\prime}(j=1,2)$ of ${ }^{\diamond} E$ which induce $u_{j}$. Then it can be shown that $\left(\chi^{a} \cdot h_{0}\left(u_{1}^{\prime}, u_{2}^{\prime}\right)\right)_{\mid D_{1}^{\circ}}$ is independent of the choice of $u_{j}^{\prime}$, which is denoted by $h_{a, \alpha}\left(u_{1}, u_{2}\right)$.

We have the frame $\mathbf{v}_{(a, \alpha)}$ of ${ }^{i} \mathrm{Gr}_{a, \alpha}^{F, \mathbb{E}}\left({ }^{\diamond} E\right)$ induced by $\mathbf{v}$ in Section 3.1.1. By construction,

$$
h_{a, \alpha}\left(v_{i}, v_{j}\right)=\rho^{a} \cdot \delta_{i, j} \cdot\left|z_{2}\right|^{-2 a_{2}\left(v_{i}\right)} .
$$

Hence, the following equality can be checked by a direct calculation:

$$
\operatorname{tr} R\left(h_{a, \alpha}\right)-a \cdot \operatorname{rank} \operatorname{Gr}_{a, \alpha}^{F, \mathbb{E}}\left({ }^{\diamond} E\right) \cdot \bar{\partial} \partial \log \rho=0
$$

Let $F_{0}$ denote the $C^{\infty}$-section of $\operatorname{End}\left({ }^{\diamond} E\right) \otimes \Omega_{X}^{1,0}(\log D)$ determined by $F_{0}\left(v_{j}\right)=$ $v_{j} \cdot \alpha_{1}\left(v_{j}\right) \cdot \partial \log \chi$. Then $\mathbb{D}^{\lambda}-F_{0}$ is $C^{\infty}$ around $D_{1}^{\circ}$, whose restriction preserves the filtration ${ }^{1} F$ and the decomposition ${ }^{1} \mathbb{E}$. Hence, we obtain an induced $\lambda$-connection $\mathbb{D}_{a, \alpha}^{\lambda}$ of ${ }^{1} \operatorname{Gr}_{(a, \alpha)}^{F, \mathbb{E}}\left({ }^{\diamond} E\right)$. We have $\theta_{a, \alpha}$ induced by $\mathbb{D}_{a, \alpha}^{\lambda}$ and $h_{a, \alpha}$.

Lemma 3.4 The following holds:

$$
\bar{\partial} \operatorname{tr} \theta_{a, \alpha}+\frac{\lambda a+\alpha}{1+|\lambda|^{2}} \operatorname{rank}\left({ }^{1} \mathrm{Gr}_{a, \alpha}^{F, \mathbb{E}}\left({ }^{\diamond} E\right)\right) \cdot \bar{\partial} \partial \log \rho=0
$$

Proof Let $\mathbb{D}_{a, \alpha, 1}^{\lambda}$ and $\theta_{a, \alpha, 1}$ denote the operator, and let $h_{a, \alpha, 1}$ denote the metric in the case where $\rho$ is constantly 1 . Since $\theta_{a, \alpha, 1}$ is holomorphic, we have $\bar{\partial} \operatorname{tr} \theta_{a, \alpha, 1}=0$. Note that we have $\mathbb{D}_{a, \alpha}^{\lambda}=\mathbb{D}_{a, \alpha, 1}^{\lambda}-\alpha \cdot \partial \log \rho$ and $h_{a, \alpha}=h_{a, \alpha, 1} \cdot \rho^{a}$. Then we obtain $\theta_{a, \alpha, 1}=\theta_{a, \alpha}+\left(1+|\lambda|^{2}\right)^{-1}(\lambda \cdot a+\alpha) \cdot \partial \log \rho$. Thus, we obtain (24). 


\subsection{Around the smooth part of the divisor}

3.2.1 Construction of the metric and some estimates Let $X:=\Delta^{2}$ and $D:=$ $\left\{z_{1}=0\right\}$. Let $\rho$ be a positive $C^{\infty}$-function on $X$, and we put $\chi:=\rho \cdot\left|z_{1}\right|^{2}$. Let $\left(\mathbf{E}_{*}, \mathbb{D}^{\lambda}\right)$ be a graded semisimple regular filtered $\lambda$-flat bundle on $(X, D)$ satisfying the SPW-condition. We take $c \in \mathbf{R}$ such that $c \notin \mathcal{P}$ ar $\left(\mathbf{E}_{*}\right)$. By the assumption, there exist a positive integer $m$ and real numbers $\gamma$ with $-1 / m<\gamma \leq 0$ such that

$$
\mathcal{P a r}\left({ }_{c} E_{*}\right) \subset\{c+\gamma+p / m \mid p \in \mathbf{Z},-1<\gamma+p / m<0\} .
$$

Let $\tilde{X}:=\Delta_{\zeta}^{2}$ and $\tilde{D}:=\left\{\zeta_{1}=0\right\}$. Let $\varphi: \tilde{X} \longrightarrow X$ be given by $\varphi\left(\zeta_{1}, \zeta_{2}\right)=\left(\zeta_{1}^{m}, \zeta_{2}\right)$. We have the induced filtered $\lambda$-flat bundle $\left(\widetilde{\mathbf{E}}_{*}, \widetilde{\mathbb{D}}^{\lambda}\right)$ on $(\tilde{X}, \widetilde{D})$ as in Section 3.1.1. We put $\tilde{c}:=m \cdot(c+\gamma)$. Then $\operatorname{Par}\left(\tilde{c} \widetilde{E}_{*}\right)$ is contained in $\{\tilde{c}+p \mid p \in \mathbf{Z}\}$.

We have the generalized eigen decomposition ${ }_{c} E_{\mid D}=\bigoplus \mathbb{E}_{\alpha}$. We have the filtration $F$ of ${ }_{c} E_{\mid D}$. Let $\mathbf{v}$ be a frame of ${ }_{c} E$ compatible with $F$ and $\mathbb{E}$. We put $a\left(v_{j}\right):=\operatorname{deg}^{F}\left(v_{j}\right)-(c+\gamma)$. Let $\alpha\left(v_{j}\right) \in \mathbf{C}$ be determined by $v_{j \mid D} \in \mathbb{E}_{\alpha\left(v_{j}\right)}$. We put $\tilde{v}_{j}:=\varphi^{*}\left(v_{j}\right) \cdot \zeta_{1}^{m \cdot a\left(v_{j}\right)}$. Then $\widetilde{\mathbf{v}}=\left(\widetilde{v}_{j}\right)$ gives the frame of $\tilde{c} \widetilde{E}$. Let $\Gamma$ be the diagonal matrix whose $(j, j)$-entries are given by the following:

$$
\alpha\left(v_{j}\right) \cdot \partial \log \left(\varphi^{*} \chi\right)+\lambda \cdot m \cdot a\left(v_{j}\right) \cdot \frac{d \zeta_{1}}{\zeta_{1}}
$$

Let $A$ be determined by $\widetilde{\mathbb{D}}^{\lambda} \widetilde{\mathbf{v}}=\widetilde{\mathbf{v}} \cdot A$, and let $A_{0}:=A-\Gamma$. Let $F_{\Gamma}$ be the $C^{\infty}$-section of $\operatorname{End}(\widetilde{E}) \otimes \Omega^{1}$ on $\widetilde{X}-\widetilde{D}$, determined by $F_{\Gamma} \widetilde{\mathbf{v}}=\widetilde{\mathbf{v}} \Gamma$. We put $\widetilde{\mathbb{D}}_{0}:=\widetilde{\mathbb{D}}-F_{\Gamma}$.

Let $A_{0}=A_{0}^{1} \cdot d \zeta_{1}+A_{0}^{2} \cdot d \zeta_{2}$. If $m$ is sufficiently large, the following holds:

(A) $A_{0}^{1}=O\left(\left|\zeta_{1}\right|^{2}\right)$. Moreover, $\left(A_{0}^{2}\right)_{k, l}=O\left(\left|\zeta_{1}\right|^{2}\right)$ in the case $\left(a\left(v_{k}\right), \alpha\left(v_{k}\right)\right) \neq$ $\left(a\left(v_{l}\right), \alpha\left(v_{l}\right)\right)$.

Let $\tilde{h}_{1}$ be a $\operatorname{Gal}(\tilde{X} / X)$-equivariant hermitian metric of ${ }^{\diamond} \widetilde{E}$ such that $\tilde{h}_{1}\left(v_{i}, v_{j}\right)=$ $O\left(\left|\zeta_{1}\right|^{2}\right)$ if $\left(a\left(v_{i}\right), \alpha\left(v_{i}\right)\right) \neq\left(a\left(v_{j}\right), \alpha\left(v_{j}\right)\right)$. We set $\tilde{h}:=\varphi^{*}\left(\chi^{-c-\gamma}\right) \cdot \tilde{h}_{1}$.

Let $\tilde{\theta}$ (resp. $\tilde{\theta}_{0}$ ) be the section of $\operatorname{End}(\tilde{E}) \otimes \Omega^{1}$ on $\tilde{X}-\widetilde{D}$ induced by $\tilde{h}$ and $\widetilde{\mathbb{D}}^{\lambda}$ (resp. $\widetilde{\mathbb{D}}_{0}^{\lambda}$ ) as in Section 2.2.1. Let $\widetilde{\theta}^{\dagger}$ and $\widetilde{\theta}_{0}^{\dagger}$ denote the adjoint of $\tilde{\theta}$ and $\tilde{\theta}_{0}$, respectively. Denote the Euclidean metric of $\tilde{X}$ by $\widetilde{g}$.

\section{Lemma 3.5}

- $\left[\widetilde{\theta}, \tilde{\theta}^{\dagger}\right]$ is bounded with respect to $\tilde{h}$ and $\tilde{g}$.

- $\tilde{\theta}^{2}=O\left(\left|z_{1}\right|\right) \cdot d z_{1} \cdot d z_{2}$. 
Proof Similar to Lemma 3.1.

Lemma 3.6 We have the boundedness of $G\left(\widetilde{\mathbb{D}}^{\lambda}, \tilde{h}\right)$ and $\tilde{\theta}^{2} \cdot \tilde{\theta}^{\dagger}$ with respect to $\tilde{h}$ and $\tilde{g}$.

Proof It follows from Lemma 3.5. See the proof of Lemma 3.2.

We have the induced hermitian metric $h$ of $E$ on $X-D$. It is adapted to the parabolic structure of $E$. Let $\theta$ denote the section of $\operatorname{End}(E) \otimes \Omega_{X-D}^{1}$ induced by $h$ and $\mathbb{D}^{\lambda}$, and let $\theta^{\dagger}$ denote the adjoint. Let $g_{m}$ denote the metric of $X-D$ given by $g_{m}=\left|z_{1}\right|^{-2+2 / m} \cdot d z_{1} \cdot d \bar{z}_{1}+d z_{2} \cdot d \bar{z}_{2}$.

Corollary 3.7 We have the boundedness of $G\left(\mathbb{D}^{\lambda}, h\right)$ and $\theta^{2} \cdot \theta^{\dagger}$ with respect to $h$ and $g_{m}$.

3.2.2 The induced metric and the $\lambda$-connections on the divisors For simplicity, we assume $c=\gamma=0$ in this subsection. Let $(a, \alpha) \in \mathcal{K} \mathcal{M S}\left({ }^{\diamond} E_{*}\right)$. We study the induced hermitian metric and the induced $\lambda$-connection of $\operatorname{Gr}_{a, \alpha}^{F, \mathbb{E}}\left({ }^{\diamond} E\right)$. Let $u_{j}$ $(j=1,2)$ be sections of $\operatorname{Gr}_{a, \alpha}^{F, \mathbb{E}}\left({ }^{\diamond} E\right)$. We take sections $u_{j}^{\prime}(j=1,2)$ of ${ }^{\diamond} E$ which induce $u_{j}$. Then it can be shown that $\left(\chi^{a} \cdot h_{0}\left(u_{1}^{\prime}, u_{2}^{\prime}\right)\right)_{\mid D}$ is independent of the choice of $u_{j}^{\prime}$, which is denoted by $h_{a, \alpha}\left(u_{1}, u_{2}\right)$.

On the other hand, let $U_{a, \alpha}$ be the subbundle of ${ }^{\diamond} \widetilde{E}$ generated by $\tilde{v}_{j}$ such that $\left(a\left(v_{j}\right), \alpha\left(v_{j}\right)\right)=(a, \alpha)$. It is easy to see that the restriction $U_{a, \alpha \mid \tilde{D}}$ are independent of the choice of the frame $\mathbf{v}$, and $U_{a, \alpha \mid \widetilde{D}}$ are orthogonal with respect to $\widetilde{h}_{\mid \widetilde{D}}$. The induced metric of $U_{a, \alpha \mid \tilde{D}}$ is denoted by $h_{a, \alpha}^{\prime}$.

Lemma 3.8 Let $R\left(h_{a, \alpha}\right)$ and $R\left(h_{a, \alpha}^{\prime}\right)$ denote the curvatures of $\left(\operatorname{Gr}_{a, \alpha}^{F, \mathbb{E}}(E), h_{a, \alpha}\right)$ and $\left(U_{a, \alpha \mid \widetilde{D}}, h_{a, \alpha}^{\prime}\right)$. Then we have the following relation:

$$
\operatorname{tr}\left(R\left(h_{a, \alpha}^{\prime}\right)\right)=\operatorname{tr}\left(R\left(h_{a, \alpha}\right)\right)-a \cdot \operatorname{rank} \operatorname{Gr}_{a, \alpha}^{F, \mathbb{E}}(E) \cdot \bar{\partial} \partial \log \rho
$$

Proof We take the isomorphism $\Phi: \operatorname{Gr}_{a, \alpha}^{F, \mathbb{E}}(E) \simeq U_{a, \alpha \mid \widetilde{D}}$ given as follows. Let $v$ be a section of $\operatorname{Gr}_{a, \alpha}^{F, \mathbb{E}}(E)$. Let $v^{\prime}$ be a section of ${ }^{\diamond} E$ which induces $v$. Then $\Phi(v):=\left(\varphi^{*}\left(v^{\prime}\right) \cdot z_{1}^{m \cdot a}\right)_{\mid \widetilde{D}}$ is contained in $U_{a, \alpha \mid}$, and independent of the choice of $v^{\prime}$. Under the isomorphism, we have $h_{a, \alpha}^{\prime}=h_{a, \alpha} \cdot \rho^{-a}$. Then (25) follows.

We have the induced $\lambda$-connection, once we fix $\chi$. (See Biquard [1].) Let $f$ be any section of $\operatorname{Gr}_{a, \alpha}^{F, \mathbb{E}}(E)$. Let $\tilde{f}$ be a lift of $f$ to ${ }^{\diamond} E$. We put $\mathbb{D}^{\lambda} f-\alpha \cdot \log \chi \cdot f=$ : $G_{1} \cdot\left(d z_{1} / z_{1}\right)+G_{2} \cdot d z_{2}$. Then $G_{1 \mid D}$ is contained in $F_{<a}(E)$. Hence, $G_{2} \cdot d z_{2}$ induces the well defined section of $\operatorname{Gr}_{a, \alpha}^{F, \mathbb{E}}(E) \otimes \Omega_{D}^{1}$, which is $\mathbb{D}_{a, \alpha}(f)$. We have the induced section $\theta_{a, \alpha}$ of $\operatorname{End}\left(\mathrm{Gr}_{a, \alpha}^{F, \mathbb{E}}(E)\right) \otimes \Omega_{D}^{1}$. 
Lemma 3.9 We have the following relation:

$$
\operatorname{tr}\left(R\left(h_{a, \alpha}^{\prime}\right)\right)=-\frac{1+|\lambda|^{2}}{\lambda}\left(\bar{\partial} \operatorname{tr} \theta_{a, \alpha}+\frac{(a \lambda+\alpha) \cdot \bar{\partial} \partial \log \rho}{1+|\lambda|^{2}} \operatorname{rank} \operatorname{Gr}_{a, \alpha}^{F, \mathbb{E}}(E)\right)
$$

Proof We have the relation:

$$
R(\tilde{h})=-\frac{1+|\lambda|^{2}}{\lambda} d^{\prime \prime} \tilde{\theta}=-\frac{1+|\lambda|^{2}}{\lambda}\left(d^{\prime \prime} \tilde{\theta}_{0}+\frac{1}{1+|\lambda|^{2}} d^{\prime \prime} F_{\Gamma}\right)
$$

Let $\mathbb{D}_{a, \alpha}^{\lambda \prime}$ be the induced $\lambda$-connection of $U_{a, \alpha \mid \tilde{D}}$, and let $\theta_{a, \alpha}^{\prime}$ be the section of $\operatorname{End}\left(U_{a, \alpha \mid} \mid \widetilde{D}\right) \otimes \Omega_{\widetilde{D}}^{1}$ induced by $\mathbb{D}_{a, \alpha}^{\lambda \prime}$ and $h_{a, \alpha}^{\prime}$. Then we obtain the following equality from (27):

$$
\operatorname{tr}\left(R\left(h_{a, \alpha}^{\prime}\right)\right)=-\frac{1+|\lambda|^{2}}{\lambda}\left(\bar{\partial} \operatorname{tr} \theta_{a, \alpha}^{\prime}+\frac{1}{1+|\lambda|^{2}} \cdot \alpha \cdot \bar{\partial} \partial \log \rho \cdot \operatorname{rank} \operatorname{Gr}_{a, \alpha}^{F, \mathbb{E}}(E)\right)
$$

Under the isomorphism $\Phi$ in the proof of Lemma 3.8, we have $\mathbb{D}_{a, \alpha}^{\lambda \prime}=\mathbb{D}_{a, \alpha}^{\lambda}$. Because $h_{a, \alpha}^{\prime}=h_{a, \alpha} \cdot \rho^{-a}$, we have $\theta_{a, \alpha}^{\prime}=\theta_{a, \alpha}+a \lambda\left(1+|\lambda|^{2}\right)^{-1} \partial \log \rho$. Therefore, the right hand side of (28) is the same as (26).

\subsection{An ordinary metric}

3.3.1 Setting Let $X$ be a smooth projective surface, and let $D$ be a simple normal crossing hypersurface with the irreducible decomposition $D=\bigcup_{i \in S} D_{i}$. Let $L$ be an ample line bundle on $X$, and $\omega$ be a Kahler form which represents $c_{1}(L)$. We take a hermitian metric $g_{i}$ of $\mathcal{O}\left(D_{i}\right)$. The canonical section $\mathcal{O} \longrightarrow \mathcal{O}\left(D_{i}\right)$ is denoted by $\sigma_{i}$. Let $\epsilon$ be any number such that $0<\epsilon<1 / 2$. Let us fix a sufficiently large number $N$, for example $N>10$. For some positive number $C>0$, we put

$$
\omega_{\epsilon}:=\omega+\sum_{i} C \cdot \epsilon^{N} \cdot \sqrt{-1} \partial \bar{\partial}\left|\sigma_{i}\right|_{g_{i}}^{2 \epsilon} .
$$

It can be shown that $\omega_{\epsilon}$ are Kahler metrics of $X-D$ for any $0<\epsilon<1 / 2$, if $C$ is sufficiently small.

Remark 3.10 The factor $\epsilon^{N}$ is added for the argument in Section 5.1 to use Proposition 2.16 of [14].

Remark 3.11 Let $\tau$ be a closed 2-form on $X-D$ which is bounded with respect to $\omega_{\epsilon}$. Then the following formula holds:

$$
\int_{X-D} \omega \cdot \tau=\int_{X-D} \omega_{\epsilon} \cdot \tau
$$


In particular, we also have $\int_{X-D} \omega^{2}=\int_{X-D} \omega_{\epsilon}^{2}$. We will implicitly use it.

In the case $\epsilon=1 / m$ for some positive integer $m$, it can be shown that the metric $\omega_{\epsilon}$ satisfies Condition 2.33. By construction, the Kahler forms $\omega_{\epsilon}$ behave as follows around any point of $D$ :

- Let $P$ be any point of $D_{i} \cap D_{j}$, and $\left(U_{P}, z_{i}, z_{j}\right)$ be a holomorphic coordinate around $P$ such that $D_{i} \cap U_{P}=\left\{z_{i}=0\right\}$ and $D_{j} \cap U_{P}=\left\{z_{j}=0\right\}$. Then there exist positive constants $C_{i}(i=1,2)$ such that the following holds on $U_{P}$, for any $0<\epsilon<1 / 2$ :

$$
\begin{aligned}
C_{1} \cdot \omega_{\epsilon} & \leq \sqrt{-1} \cdot \epsilon^{N+2} \cdot\left(\frac{d z_{i} \cdot d \bar{z}_{i}}{\left|z_{i}\right|^{2-2 \epsilon}}+\frac{d z_{j} \cdot d \bar{z}_{j}}{\left|z_{j}\right|^{2-2 \epsilon}}\right)+\sqrt{-1}\left(d z_{i} \cdot d \bar{z}_{i}+d z_{j} \cdot d \bar{z}_{j}\right) \\
& \leq C_{2} \cdot \omega_{\epsilon}
\end{aligned}
$$

- Let $Q$ be any point of $D_{i} \backslash \bigcup_{j \neq i} D_{j}$, and $\left(U, w_{1}, w_{2}\right)$ be a holomorphic coordinate around $Q$ such that $U \cap D_{i}=\left\{w_{1}=0\right\}$. Then there exist positive constants $C_{i}(i=1,2)$ such that the following holds for any $0<\epsilon<1 / 2$ on $U$ :

$$
\begin{aligned}
C_{1} \cdot \omega_{\epsilon} & \leq \sqrt{-1} \cdot \epsilon^{N+2} \cdot\left(\frac{d w_{1} \cdot d \bar{w}_{1}}{\left|w_{1}\right|^{2-2 \epsilon}}\right)+\sqrt{-1}\left(d w_{1} \cdot d \bar{w}_{1}+d w_{2} \cdot d \bar{w}_{2}\right) \\
& \leq C_{2} \cdot \omega_{\epsilon}
\end{aligned}
$$

3.3.2 Construction and some property Let $\left(\mathbf{E}_{*}, \mathbb{D}^{\lambda}\right)$ be a graded semisimple parabolic $\lambda$-flat bundle satisfying the SPW-condition. For simplicity, we consider only the case $\lambda \neq 0$. We take $\mathbf{c} \in \mathbf{R}^{S}$ such that $c_{i} \notin \mathcal{P}$ ar $\left(\mathbf{E}_{*}, i\right)$ for each $i \in S$. Let $m$ be as in Definition 2.6, and we set $\epsilon:=m^{-1}$. Let $h_{0}$ be a $C^{\infty}$-hermitian metric of $E$ on $X-D$ as in Section 3.1 around the intersection points of $D$, and as in Section 3.2 around the smooth points of $D$. Let $\theta_{0}$ denote the section of $\operatorname{End}(E) \otimes \Omega^{1,0}$ on $X-D$ induced by $\mathbb{D}^{\lambda}$ and $h_{0}$, and let $\theta_{0}^{\dagger}$ denote the adjoint.

Lemma 3.12 We have the boundedness of $G\left(\mathbb{D}^{\lambda}, h_{0}\right)$ and $\theta_{0}^{2} \cdot \theta_{0}^{\dagger}$ with respect to $h_{0}$ and $\omega_{\epsilon}$.

Proof It follows from Corollary 3.3 and Corollary 3.7.

Corollary 3.13 The following equality holds:

$$
\int_{X-D} \operatorname{tr}\left(R\left(h_{0}\right)^{2}\right)=\frac{1}{\left(1+|\lambda|^{2}\right)^{2}} \int_{X-D} \operatorname{tr}\left(G\left(h_{0}\right)^{2}\right)
$$


As a result, we have the following formula:

$$
\left(\frac{\sqrt{-1}}{2 \pi}\right)^{2} \frac{1}{\left(1+|\lambda|^{2}\right)^{2}} \int_{X-D} \operatorname{tr}\left(G\left(h_{0}\right)^{2}\right)=2 \int_{X} \operatorname{par}^{2} \mathrm{ch}_{2}\left(\mathbf{E}_{*}\right)
$$

Proof The second equality follows from the first equality and the equality (36) in the proof of Proposition 4.18 of [14]. Due to Lemma 2.32, we have only to show the vanishing $\int \bar{\partial} \operatorname{tr}\left(\theta_{0}^{2} \cdot \theta_{0}^{\dagger}\right)=0$, which follows from the estimate of $\theta_{0}^{2} \cdot \theta_{0}^{\dagger}$ in Lemma 3.12 .

We can also show the following equality by using Lemma 4.16 of [14] and the equality $\operatorname{tr} G\left(h_{0}\right)=\left(1+|\lambda|^{2}\right) \cdot \operatorname{tr} R\left(h_{0}\right)$ :

$$
\left(\frac{\sqrt{-1}}{2 \pi}\right)^{2} \int_{X-D}\left(\frac{\operatorname{tr} G\left(h_{0}\right)}{1+|\lambda|^{2}}\right)^{2}=\left(\frac{\sqrt{-1}}{2 \pi}\right)^{2} \int_{X-D}\left(\operatorname{tr} R\left(h_{0}\right)\right)^{2}=\int_{X} \operatorname{par-c_{1}}\left(\mathbf{E}_{*}\right)^{2}
$$

Let $V \subset E$ be a $\lambda$-flat subbundle. Recall $\lambda \neq 0$. Because of the regularity, we have the saturated filtered $\lambda$-flat subsheaf $\mathbf{V}_{*} \subset \mathbf{E}_{*}$. Let $h_{V}$ be the metric of $V$ induced by $h_{0}$.

Lemma 3.14 $\operatorname{deg}_{\omega_{\epsilon}}\left(V, h_{V}\right)=$ par-deg ${ }_{\omega}\left(\mathbf{V}_{*}\right)$ holds. In particular, $\operatorname{deg}_{\omega_{\epsilon}}\left(E, h_{0}\right)=$ $\operatorname{par}-\operatorname{deg}_{\omega}\left(\mathbf{E}_{*}\right)$.

Proof It can be shown by the same argument as the proof of Lemma 4.20 of [14].

3.3.3 The induced metric and the $\lambda$-connection on the divisors For simplicity, we assume $c_{i}=\gamma_{i}=0(i \in S)$ in this subsection. We put $\mathcal{S}\left(D_{i}\right):=D_{i} \cap \bigcup_{j \neq i} D_{j}$ and $D_{i}^{\circ}:=D_{i} \backslash \mathcal{S}\left(D_{i}\right)$. Let $(a, \alpha) \in \mathcal{K} \mathcal{M S}\left({ }^{\diamond} E, \mathbf{F}, i\right)$. We have the naturally induced parabolic flat bundle ${ }^{i} \mathrm{Gr}_{a, \alpha}^{F, \mathbb{E}}\left({ }^{\diamond} E\right)_{*}$ on $\left(D_{i}, \mathcal{S}\left(D_{i}\right)\right)$. By using the functions $\left|\sigma_{i}\right|_{g_{i}}^{2}$, as explained in Section 3.2.2, we obtain the induced hermitian metric ${ }^{i} h_{a, \alpha}$ and the $\lambda-$ connection ${ }^{i} \mathbb{D}_{a, \alpha}^{\lambda}$ of $\left.{ }^{i} \mathrm{Gr}_{a, \alpha}^{F, \mathbb{E}}\left({ }^{\diamond} E\right)\right|_{\mid D_{i}^{\circ}}$. (See also Section 3.1.2.) Let $\tau_{i}:=\bar{\partial} \partial \log \left|\sigma_{i}\right|_{g_{i}}^{2}$.

Lemma 3.15 The following equality holds:

$$
\operatorname{tr}\left(R\left(h_{a, \alpha}\right)\right)=-\frac{1+|\lambda|^{2}}{\lambda} \bar{\partial} \operatorname{tr}\left({ }^{i} \theta_{a, \alpha}\right)-\lambda^{-1} \alpha \cdot \tau_{i} \cdot \operatorname{rank}^{i} \operatorname{Gr}_{a, \alpha}^{F, \mathbb{E}}\left({ }^{\diamond} E\right)
$$

Proof It follows from (23), (24), (25) and (26). 
Corollary 3.16 We have the following formulas:

(30) $\quad \operatorname{par}_{-\operatorname{deg}_{D_{i}}}\left({ }^{i} \operatorname{Gr}_{a, \alpha}^{F, \mathbb{E}}\left({ }^{\diamond} E\right)_{*}\right)=-\operatorname{Re}\left(\lambda^{-1} \alpha\right) \cdot \operatorname{rank}^{i} \operatorname{Gr}_{a, \alpha}^{F, \mathbb{E}}\left({ }^{\diamond} E\right) \cdot\left[D_{i}\right]^{2}$

$$
-\sum_{P \in \mathcal{S}\left(D_{i}\right)}\left(\operatorname{Re}\left(\lambda^{-1} \operatorname{tr}\left(\operatorname{Res} P\left({ }^{i} \mathbb{D}_{a, \alpha}^{\lambda}\right)\right)\right)+\mathrm{wt}^{i}\left(\mathrm{Gr}_{a, \alpha}^{F, \mathbb{E}}\left({ }^{\diamond} E\right)_{*}, P\right)\right)
$$

(31) $0=\sum_{P \in \mathcal{S}\left(D_{i}\right)} \operatorname{Im}\left(\lambda^{-1} \operatorname{tr}\left(\operatorname{Res}_{P}\left({ }^{i} \mathbb{D}_{a, \alpha}^{\lambda}\right)\right)\right)+\operatorname{Im}\left(\lambda^{-1} \alpha\right) \cdot \operatorname{rank}^{i} \operatorname{Gr}_{a, \alpha}^{F, \mathbb{E}}\left({ }^{\diamond} E\right) \cdot\left[D_{i}\right]^{2}$

Proof It follows from Lemma 2.57 and Lemma 3.15.

Remark 3.17 Although we have assumed that graded semisimplicity and the SPWcondition for $\left(\mathbf{E}_{*}, \mathbb{D}^{\lambda}\right)$, the formulas (30) and (31) without the assumption, because the general case can be reduced to the above special case by using $\epsilon$-perturbations explained in Section 2.1.6.

\subsection{Preliminary existence result for a Hermitian-Einstein metric}

Let $X$ be a smooth irreducible projective surface with an ample line bundle $L$ and a simple normal crossing divisor $D$. Let $\omega$ be a Kahler form representing $c_{1}(L)$. Let $\left(\mathbf{E}_{*}, \mathbb{D}^{\lambda}\right)$ be a graded semisimple regular filtered $\lambda$-flat bundle on $(X, D)$. We assume the SPW-condition in Section 3.3.2. Let $m$ be as in Definition 2.6, and we set $\epsilon:=m^{-1}$. Let $\omega_{\epsilon}$ be the Kahler metric given in (29). We have an ordinary metric $h_{0}$ constructed in Section 3.3.2. We also use the other notation in Section 3.3.

Lemma 3.18 We can construct a hermitian metric $h_{i n}$ for $E_{\mid X-D}$ which satisfies the following conditions:

- $h_{i n}$ is adapted to $\mathbf{E}_{*}$. More strongly, $h_{i n}=h_{0} \cdot e^{\chi}$ for some function $\chi$ such that $\chi, \partial \chi$ and $\bar{\partial} \partial \chi$ are bounded with respect to $\omega_{\epsilon}$.

- $G\left(h_{i n}, \mathbb{D}^{\lambda}\right)$ is bounded with respect to $h_{i n}$ and $\omega_{\epsilon}$.

- Let $\mathbf{V}_{*}$ be a $\lambda$-flat filtered subsheaf of $\mathbf{E}_{*}$. Let $V:=\mathbf{V}_{\mid X-D}$ and let $h_{i n, V}$ denote the induced metric of $V$. Then par- $\operatorname{deg}_{\omega}\left(\mathbf{V}_{*}\right)=\operatorname{deg}_{\omega_{\epsilon}}\left(V, h_{i n, V}\right)$ holds.

- $\left(1+|\lambda|^{2}\right)^{-1} \operatorname{tr} G\left(h_{i n}, \mathbb{D}^{\lambda}\right) \cdot \omega_{\epsilon}=a \cdot \omega_{\epsilon}^{2}$ for some constant $a$. The constant $a$ is determined by the following condition:

$$
a \cdot \frac{\sqrt{-1}}{2 \pi} \frac{\operatorname{rank} E}{2} \int_{X-D} \omega_{\epsilon}^{2}=a \cdot \frac{\sqrt{-1}}{2 \pi} \frac{\operatorname{rank} E}{2} \int_{X} \omega^{2}={\operatorname{par}-\operatorname{deg}_{\omega}}_{\left(\mathbf{E}_{*}\right) .}
$$


- The following equalities hold:

$$
\begin{aligned}
& \left(\frac{\sqrt{-1}}{2 \pi}\right)^{2} \int_{X-D} \frac{\operatorname{tr}\left(G\left(h_{i n}\right)^{2}\right)}{\left(1+|\lambda|^{2}\right)^{2}}=\int_{X} 2 \operatorname{par}^{-c_{2}}\left(\mathbf{E}_{*}\right)
\end{aligned}
$$

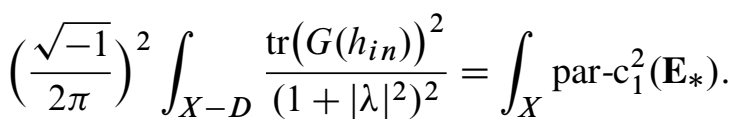

- Let $s$ be determined by $h_{i n}=h_{0} \cdot s$. Then $s$ and $s^{-1}$ are bounded, and $\mathbb{D}^{\lambda} s$ is $L^{2}$ with respect to $h_{0}$ and $\omega_{\epsilon}$.

Due to the third condition, $\left(E, h_{i n}, \theta\right)$ is analytic stable with respect to $\omega_{\epsilon}$, if and only if $\left(\mathbf{E}_{*}, \mathbb{D}^{\lambda}\right)$ is $\mu_{L}$-stable. The metric $h_{i n}$ is called an initial metric.

Proof By a consideration of orbifolds, we can take a real-valued bounded function $\chi$ such that (i) $\partial \chi$ and $\partial \bar{\partial} \chi$ are bounded with respect to $\omega_{\epsilon}$, (ii) $\Delta_{\omega_{\epsilon}} \chi=\sqrt{-1} a-$ $\sqrt{-1} \operatorname{rank}(E)^{-1} \Lambda_{\omega_{\epsilon}} \operatorname{tr} R\left(h_{0}\right)$. We put $h_{i n}:=h_{0} \cdot e^{\chi}$. By construction, the fourth condition is satisfied. The other property can be reduced to the property for $h_{0}$, as in Lemma 6.3 of [14].

Proposition 3.19 If $\left(\mathbf{E}_{*}, \mathbb{D}^{\lambda}, h\right)$ is $\mu_{L}$-stable, there exists a hermitian metric $h_{\mathrm{HE}}$ of $\left(E, \mathbb{D}^{\lambda}\right)$ with respect to $\omega_{\epsilon}$ satisfying the following properties:

- Hermitian-Einstein condition $\left(1+\left|\lambda^{2}\right|\right)^{-1} \Lambda_{\omega_{\epsilon}} G\left(h_{\mathrm{HE}}\right)=a$ holds for the constant a determined by (32).

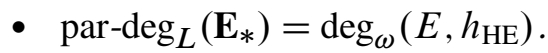

- We have the following formulas:

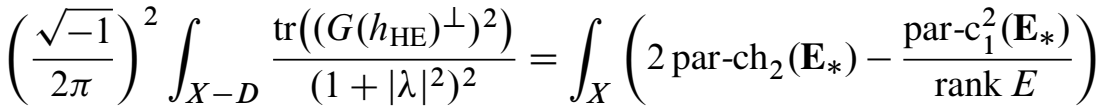

$$
\begin{aligned}
& \left(\frac{\sqrt{-1}}{2 \pi}\right)^{2} \int_{X-D} \frac{\operatorname{tr}\left(G\left(h_{\mathrm{HE}}\right)^{2}\right)}{\left(1+|\lambda|^{2}\right)^{2}}=\int_{X} 2 \operatorname{par}^{-\operatorname{ch}_{2}}\left(\mathbf{E}_{*}\right) \text {. }
\end{aligned}
$$

Here $G\left(h_{\mathrm{HE}}\right)^{\perp}$ denotes the trace free part of $G\left(h_{\mathrm{HE}}\right)$.

- $h_{\mathrm{HE}}$ is adapted to $\mathbf{E}_{*}$, ie, $\mathbf{E}_{*}\left(h_{\mathrm{HE}}\right) \simeq \mathbf{E}_{*}$. More strongly, $s$ and $s^{-1}$ are bounded with respect to $h_{i n}$, and $\mathbb{D}^{\lambda} s$ is $L^{2}$ with respect to $h_{i n}$ and $\omega_{\epsilon}$, where $s$ is determined by $h_{\mathrm{HE}}=h_{\text {in }} \cdot s$.

Proof It follows from Lemma 3.18 and Proposition 2.49. 
Bogomolov-Gieseker inequality Let $Y$ be a smooth projective variety of any dimension. Let $L$ be an ample line bundle on $Y$, and let $D$ be a simple normal crossing divisor.

Corollary 3.20 Let $\left(\mathbf{E}_{*}, \mathbb{D}^{\lambda}\right)$ be a $\mu_{L}$-stable regular filtered $\lambda$-flat bundle on $(Y, D)$ in codimension two. Then Bogomolov-Gieseker inequality holds for $\mathbf{E}_{*}$. Namely, we have the following inequality:

$$
\int_{Y} \operatorname{par}-\mathrm{ch}_{2, L}\left(\mathbf{E}_{*}\right) \leq \frac{\int_{Y} \operatorname{par}^{-c_{1, L}^{2}}\left(\mathbf{E}_{*}\right)}{2 \operatorname{rank} E} .
$$

Proof Similar to Theorem 6.1 of [14]. Namely, since we have the Mehta-Ramanathan type theorem (Proposition 2.21), we have only to prove the claim in the case $\operatorname{dim} Y=2$. Due to the method of perturbation of parabolic structure explained in Section 2.1.6, we have only to prove the inequality in the case $\left(\mathbf{E}_{*}, \mathbb{D}^{\lambda}\right)$ is a graded semisimple $\mu_{L}$-stable regular filtered $\lambda$-flat bundle on $(Y, D)$, satisfying the SPW-condition. Then we can take a Hermitian-Einstein metric $h_{\mathrm{HE}}$ as in Proposition 3.19, for which we have the standard inequality (see Proposition 3.4 of [17]):

$$
\int_{Y-D} \operatorname{tr}\left(\left(G\left(h_{\mathrm{HE}}\right)^{\perp}\right)^{2}\right) \geq 0 .
$$

Hence we obtain the desired inequality from (33).

\subsection{Some formulas and vanishings of characteristic numbers}

Let $X$ be a smooth projective surface, and let $D$ be a simple normal crossing divisor of $X$. We will derive some formulas and vanishings for the characteristic numbers of $\left(\mathbf{E}_{*}, \mathbb{D}^{\lambda}\right)$.

Remark 3.21 To begin with, we remark that we have only to show such formulas for regular filtered $\lambda$-flat bundles satisfying the following conditions, due to the method of perturbation of the parabolic structure (Section 2.1.6):

- graded semisimple, $\mathcal{P} a r\left(\mathbf{E}_{*}, i\right) \subset \mathbf{Q}$, and $0 \notin \mathcal{P} a r\left(\mathbf{E}_{*}, i\right)$ for any $i \in S$.

We will implicitly use the conditions in the following argument.

We restrict ourselves to the case $\operatorname{dim} X=2$ just for simplicity. The formula can be obviously generalized for $\int_{X} \operatorname{par}_{-c_{2, L}}\left(\mathbf{E}_{*}\right)$ of regular $\lambda$-flat parabolic bundles $\left(\mathbf{E}_{*}, \mathbb{D}^{\lambda}\right)$ on $(X, D)$ in codimension two even in the case $\operatorname{dim} X>2$, where $L$ denotes an ample line bundle on $X$. 
We introduce a notation to simplify the description. An element $(a, \alpha) \in \mathcal{K} \mathcal{M S}(i):=$ $\mathcal{K} \mathcal{M S}\left({ }^{\diamond} E, i\right)$ is described as $u$, and we put

$$
r(i, u):=\operatorname{rank}_{D_{i}}\left({ }^{i} \operatorname{Gr}_{u}^{F, \mathbb{E}}\left({ }^{\diamond} E\right)\right)
$$

For any point $P \in D_{i} \cap D_{j}$ and $\left(u_{i}, u_{j}\right) \in \mathcal{K} \mathcal{M S}(P):=\mathcal{K} \mathcal{M S}\left({ }^{\diamond} E, P\right)$, we put

$$
r\left(P, u_{i}, u_{j}\right):=\operatorname{rank}\left({ }^{P} \mathrm{Gr}_{u_{i}, u_{j}}^{F, \mathbb{E}}(E)\right)
$$

The self-intersection number of $D_{i}$ is denoted by $\left[D_{i}\right]^{2}$.

Proposition 3.22 We have the following equality:

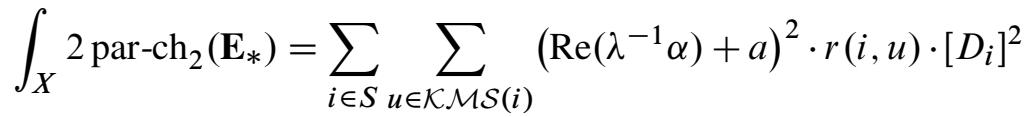

$$
\begin{aligned}
& +\sum_{i \in S} \sum_{\substack{j \neq i \\
P \in D_{i} \cap D_{j}}} \sum_{\left(u_{i}, u_{j}\right) \in \mathcal{K} \mathcal{M S}(P)}\left(\operatorname{Re} \lambda^{-1} \alpha_{i}+a_{i}\right)\left(\operatorname{Re} \lambda^{-1} \alpha_{j}+a_{j}\right) \cdot r\left(P, u_{i}, u_{j}\right)
\end{aligned}
$$

We also have the following equalities:

$$
\begin{aligned}
& \int_{X} 2 \operatorname{par}-\operatorname{ch}_{2}\left(\mathbf{E}_{*}\right) \\
& =\sum_{i \in S} \sum_{u \in \mathcal{K} \mathcal{M} \mathcal{S}(i)} \operatorname{Re}\left(\lambda^{-1} \alpha+a\right) \cdot\left(-\operatorname{par}^{-\operatorname{deg}_{D_{i}}}\left({ }^{i} \operatorname{Gr}_{a, \alpha}^{F, \mathbb{E}}\left({ }^{\diamond} E\right)_{*}\right)+a \cdot r(i, u) \cdot\left[D_{i}\right]^{2}\right) \\
& \text { (36) } 0=\sum_{i \in S} \sum_{u \in \mathcal{K} \mathcal{M} S(i)} \operatorname{Im}\left(\lambda^{-1} \alpha\right) \cdot\left(-\operatorname{par}^{-\operatorname{deg}_{D_{i}}}\left({ }^{i} \operatorname{Gr}_{u}^{F, \mathbb{E}}\left({ }^{\diamond} E\right)_{*}\right)+a \cdot r(i, u) \cdot\left[D_{i}\right]^{2}\right)
\end{aligned}
$$

Proof Let $h_{0}$ be an ordinary metric for $\left(\mathbf{E}_{*}, \mathbb{D}^{\lambda}\right)$ as in Section 3.3. We use the notation there in this proof. Let $X_{\delta}:=\bigcap\left\{\left|\sigma_{i}\right| \geq \delta\right\}$ and $Y_{\delta, i}:=X_{\delta} \cap\left\{\left|\sigma_{i}\right|=\delta\right\}$. We have

$$
R\left(h_{0}\right)=-\lambda^{-1}\left(1+|\lambda|^{2}\right) \cdot d^{\prime \prime} \theta_{0} .
$$

Hence, we have the following equality:

$$
\begin{aligned}
\lim _{\delta \rightarrow 0}\left(\frac{\sqrt{-1}}{2 \pi}\right)^{2} \int_{X_{\delta}} \operatorname{tr}\left(R\left(h_{0}\right)^{2}\right) & \\
= & -\frac{1+|\lambda|^{2}}{\lambda} \lim _{\delta \rightarrow 0}\left(\frac{\sqrt{-1}}{2 \pi}\right)^{2} \int_{\partial X_{\delta}} d \operatorname{tr}\left(\theta_{0} \cdot R\left(h_{0}\right)\right)
\end{aligned}
$$


By using the estimates in Sections 3.1-3.2, the contribution of $Y_{\delta, i}$ to (37) is the following:

$$
\begin{aligned}
-\frac{1}{m} \sum_{(a, \alpha) \in \mathcal{K} \mathcal{M S}(i)} m\left(a+\lambda^{-1} \alpha\right) \frac{\sqrt{-1}}{2 \pi} \int_{D_{i}}\left(\operatorname{tr}\left(R\left({ }^{i} h_{a, \alpha}\right)\right)-a \cdot r(i,(a, \alpha)) \cdot \tau_{i}\right) \\
=-\sum_{(a, \alpha) \in \mathcal{K} \mathcal{M S}(i)}\left(a+\lambda^{-1} \alpha\right) \cdot\left(\operatorname{deg}_{D_{i}}\left({ }^{i} \operatorname{Gr}_{a, \alpha}^{F, \mathbb{E}}\left({ }^{\diamond} E\right)_{*}\right)-a \cdot r(i,(a, \alpha)) \cdot\left[D_{i}\right]^{2}\right)
\end{aligned}
$$

By taking the real part, we obtain (35). By taking the imaginary part, we obtain (36). The equality (34) follows from (35) and Corollary 3.16 by a formal calculation.

Let $\left(D_{i}, \omega\right)$ denote the intersection number of $D_{i}$ and $\omega$.

Lemma 3.23 For any $C^{\infty} 2$-form $\tau$, we have the following:

$$
\begin{aligned}
\int_{X} \operatorname{par}^{-c_{1}\left(\mathbf{E}_{*}\right) \cdot \tau} & =\frac{\sqrt{-1}}{2 \pi} \int_{X} \operatorname{tr} R\left(h_{0}\right) \cdot \tau \\
& =-\sum_{i \in S} \sum_{(a, \alpha) \in \mathcal{K M S}(i)} \operatorname{Re}\left(\lambda^{-1} \alpha+a\right) \cdot r(i,(a, \alpha)) \cdot\left(D_{i}, \tau\right)
\end{aligned}
$$

Namely, the cohomology class of $\sqrt{-1}(2 \pi)^{-1} \operatorname{tr} R\left(h_{0}\right)$ is par-c $\mathbf{c}_{1}\left(\mathbf{E}_{*}\right)$. In particular, we also have the following equality:

$$
\operatorname{par}-\operatorname{deg}_{\omega}\left(\mathbf{E}_{*}\right)=-\sum_{i \in S} \sum_{(a, \alpha) \in \mathcal{K} \mathcal{M S}(i)} \operatorname{Re}\left(\lambda^{-1} \alpha+a\right) \cdot r(i,(a, \alpha)) \cdot\left(D_{i}, \omega\right)
$$

Proof Recall $R\left(h_{0}\right)=\lambda^{-1}\left(1+|\lambda|^{2}\right) \cdot d^{\prime \prime} \theta_{0}$. We obtain (38) by using the estimates in Sections 3.1-3.2.

Remark 3.24 We considered the KMS-spectra of ${ }^{\diamond} E$. But, we have the following equality for any $\mathbf{c} \in \mathbf{R}^{S}$ and $i \in S$ :

$$
\left\{\operatorname{Re}\left(\lambda^{-1} \alpha\right)+a \mid(a, \alpha) \in \mathcal{K} \mathcal{M S}\left({ }^{\diamond} E, i\right)\right\}=\left\{\operatorname{Re}\left(\lambda^{-1} \alpha\right)+a \mid(a, \alpha) \in \mathcal{K} \mathcal{M S}\left({ }_{\mathbf{c}} E, i\right)\right\}
$$

We also have such comparison for $\mathcal{K} \mathcal{M S}\left({ }^{\diamond} E, P\right)$ and $\mathcal{K} \mathcal{M S}\left({ }_{\mathbf{c}} E, P\right)$ for $\mathbf{c} \in \mathbf{R}^{S}$ and $P \in D_{i} \cap D_{j}$. Namely, the choice ${ }^{\diamond} E$ is not essential. (See also Section 6.)

Recall the formulas for $\int_{X}$ par-ch ${ }_{2}\left(\mathbf{E}_{*}\right)$ and $\operatorname{par}-\operatorname{deg}_{\omega}\left(\mathbf{E}_{*}\right)$ in Proposition 3.22 and Lemma 3.23, respectively. Then we immediately obtain the following corollary.

Corollary 3.25 If $a+\operatorname{Re} \lambda^{-1} \alpha=0$ hold for any element of the KMS-spectrum $(a, \alpha)$ of $\left(\mathbf{E}_{*}, \mathbb{D}^{\lambda}\right)$, the characteristic numbers par- $\operatorname{deg}_{\omega}\left(\mathbf{E}_{*}\right)$ and $\int_{X} \operatorname{par}-\mathrm{ch}_{2}\left(\mathbf{E}_{*}\right)$ automatically vanish. 
One of the motivation of this study is to understand the Corlette-Jost-Zuo metric from the viewpoint of the Kobayashi-Hitchin correspondence. For that purpose, we need vanishing of $\int_{X}$ par-ch ${ }_{2}\left(\mathbf{E}_{*}\right)$ as in the corollary, which is "the obstruction on the way from harmonicity to pluri-harmonicity". Corollary 3.25 clarifies this point, which we will revisit in Section 5.3.3.

\section{Continuity of some families of harmonic metrics}

In this section, we will show continuity of two kinds of families of harmonic metrics on curves, ie, Proposition 4.1 and Proposition 4.2. We will give a detailed proof of the first one. Because the second one can be proved similarly and more easily, we just give some remarks in the end of this section.

We have explained in Section 1.2.3 the role of Proposition 4.1 which plays for the proof of our main result. We will use Proposition 4.2 in Section 5.2.5 to show that some hermitian metric is $C^{1}$.

\subsection{Statements}

4.1.1 Continuity for $\epsilon$-perturbation Let $C$ be a smooth projective curve with a finite subset $D$. Let $\left(E, \mathbf{F}, \mathbb{D}^{\lambda}\right)$ be a stable parabolic flat $\lambda$-connection over $(C, D)$ with par-deg $(E, \mathbf{F})=0$. Let $\mathbf{F}^{(\epsilon)}$ be the $\epsilon$-perturbation of the parabolic structures, explained in (II) of Section 2.1.6. We remark $\operatorname{det}(E, \mathbf{F})=\operatorname{det}\left(E, \mathbf{F}^{(\epsilon)}\right)$. We fix a harmonic metric $h_{\operatorname{det}(E)}$ for $\operatorname{det}\left(E, \mathbf{F}, \mathbb{D}^{\lambda}\right)$, ie, $h_{\operatorname{det}(E)}$ is a harmonic metric of $\operatorname{det}\left(E, \mathbb{D}^{\lambda}\right)_{\mid X-D}$, adapted to the parabolic structure. Let $h^{(\epsilon)}$ be the harmonic metric for $\left(E, \mathbf{F}^{(\epsilon)}, \mathbb{D}^{\lambda}\right)$ such that $\operatorname{det}\left(h^{(\epsilon)}\right)=h_{\operatorname{det}(E)}$. Let $\theta^{(\epsilon)}$ denote the Higgs fields for the harmonic bundles $\left(E, \mathbb{D}^{\lambda}, h^{(\epsilon)}\right)$.

Proposition 4.1 The sequences $\left\{h^{(\epsilon)} \mid \epsilon>0\right\}$ and $\left\{\theta^{(\epsilon)}\right\}$ converge to $h^{(0)}$ and $\theta^{(0)}$ respectively, in the $C^{\infty}$-sense locally on $C-D$.

The proof is given in Section 4.5 after the preparation given in Sections 4.2-4.4.

4.1.2 Continuity for a holomorphic family Before going into the proof of Proposition 4.1 , we give a similar statement for another family. Let $\mathcal{C} \longrightarrow \Delta$ be a holomorphic family of smooth projective curve, and $\mathcal{D} \longrightarrow \Delta$ be a relative divisor. Let $\left(E, \mathbf{F}, \mathbb{D}^{\lambda}\right)$ be a parabolic flat bundle on $(\mathcal{C}, \mathcal{D})$. Let $t$ be any point of $\Delta$. We denote the fibers by $\mathcal{C}_{t}$ and $\mathcal{D}_{t}$, and the restriction of $\left(E, \mathbf{F}, \mathbb{D}^{\lambda}\right)$ to $\left(\mathcal{C}_{t}, \mathcal{D}_{t}\right)$ is denoted by $\left(E_{t}, \mathbf{F}_{t}, \mathbb{D}_{t}^{\lambda}\right)$. We assume par-deg $\left(E_{t}, \mathbf{F}_{t}\right)=0$ and the stability of $\left(E_{t}, \mathbf{F}_{t}, \mathbb{D}_{t}^{\lambda}\right)$ for each $t$. For simplicity, we also assume that we are given a pluri-harmonic metric $h_{\operatorname{det}(E)}$ for $\operatorname{det}\left(E, \mathbb{D}^{\lambda}, \mathbf{F}\right)$. 
Let $h_{H, t}$ be harmonic metrics for $\left(E_{t}, \mathbf{F}_{t}, \mathbb{D}_{t}^{\lambda}\right)$ such that $\operatorname{det}\left(h_{H, t}\right)=h_{\operatorname{det}(E) \mid \mathcal{C}_{t}}$. They give the metric $h_{H}$ of $E$. Let $\theta_{H, t}$ be the Higgs fields induced by $\left(E_{t}, \mathbb{D}_{t}^{\lambda}, h_{H, t}\right)$, which are sections of $\operatorname{End}\left(E_{t}\right) \otimes \Omega_{\mathcal{C}_{t}}^{1,0}\left(\log \mathcal{D}_{t}\right)$. They give a section $\theta_{H}$ of $\operatorname{End}(E) \otimes$ $\Omega_{\mathcal{C} / \Delta}^{1,0}(\log \mathcal{D})$, where $\Omega_{\mathcal{C} / \Delta}^{1,0}(\log \mathcal{D})$ denotes the sheaf of logarithmic relative $(1,0)-$ forms.

Proposition 4.2 $h_{H}$ and $\theta_{H}$ are continuous. Their derivatives of any degree along the fiber direction are continuous.

Since Proposition 4.2 can be proved similarly and more easily, we will not give a detailed proof. See Remark 4.16.

\subsection{Preliminary from elementary calculus}

For any $z \in \Delta^{*}=\{z \in \mathbf{C}|| z \mid<1\}$ and $\epsilon>0$, we define

$$
L_{\epsilon}(z):=\frac{|z|^{-\epsilon}-|z|^{\epsilon}}{\epsilon}, \quad K_{\epsilon}(z):=\frac{|z|^{-\epsilon}+|z|^{\epsilon}}{2}, \quad M_{\epsilon}(z):=|z|^{4 \epsilon}\left(1-\log |z|^{4 \epsilon}\right) .
$$

We also put $L_{0}(z):=-\log |z|^{2}, K_{0}(z):=1$ and $M_{0}(z):=1$. Then they are continuous with respect to $(z, \epsilon) \in \Delta^{*} \times \mathbf{R}_{\geq 0}$.

Lemma 4.3 For any $(z, \epsilon) \in \Delta^{*} \times \mathbf{R}_{\geq 0}$, we have $L_{0}(z) \leq L_{\epsilon}(z)$.

Proof We put $g(\epsilon):=a^{-\epsilon}-a^{\epsilon}+\epsilon \cdot \log a^{2}$ for $0<a<1$ and $0 \leq \epsilon$. Taking the derivative with respect to $\epsilon$, we obtain the following:

$$
g^{\prime}(\epsilon)=-\left(a^{-\epsilon}+a^{\epsilon}\right) \log a+\log a^{2}, \quad g^{\prime \prime}(\epsilon)=\left(a^{-\epsilon}-a^{\epsilon}\right)(\log a)^{2} \geq 0
$$

Because $g(0)=g^{\prime}(0)=0$, the claim of the lemma follows.

Lemma 4.4 $\left(K_{\epsilon}(z)-1\right) \cdot\left(L_{\epsilon}(z)^{2} \cdot \epsilon^{2} \cdot|z|^{\epsilon}\right)^{-1}$ are bounded on $\Delta^{*}$, independently of $\epsilon$. We also have $K_{\epsilon}(z)-1 \geq 0$.

Proof The second claim is clear. Let us check the first claim. For $0<a<1$ and $0 \leq \epsilon \leq 1$, we set

$$
g_{1}(\epsilon):=a^{-\epsilon}-2+a^{\epsilon}, \quad g_{2}(\epsilon):=\left(a^{-\epsilon}-a^{\epsilon}\right)^{2} a^{\epsilon}=a^{-\epsilon}-2 a^{\epsilon}+a^{3 \epsilon} .
$$

We have only to show that $g_{2}(\epsilon) \geq g_{1}(\epsilon)$. We put $g(\epsilon):=g_{2}(\epsilon)-g_{1}(\epsilon)=2+a^{3 \epsilon}-3 a^{\epsilon}$. By taking the derivative with respect to $\epsilon$, we obtain the following:

$$
g^{\prime}(\epsilon)=3 a^{3 \epsilon} \cdot \log a-3 a^{\epsilon} \cdot \log a=3\left(-a^{3 \epsilon}+a^{\epsilon}\right)(-\log a) \geq 0
$$

Because $g(0)=0$, we obtain $g(\epsilon) \geq 0$. Thus we are done. 
Lemma $4.5\left(1-M_{\epsilon}(z)\right) \cdot\left(L_{\epsilon}(z)^{2} \cdot \epsilon^{2} \cdot|z|^{\epsilon}\right)^{-1}$ are bounded on $\Delta^{*}$, independently of $\epsilon$. We also have $1-M_{\epsilon}(z) \geq 0$.

Proof We have only to show the following inequalities for $0<a<1$ and $0 \leq \epsilon<1$ :

$$
0 \leq 1-a^{4 \epsilon}\left(1-\log a^{4 \epsilon}\right) \leq 3\left(a^{-\epsilon}-a^{\epsilon}\right)^{2} a^{\epsilon}
$$

To show the left inequality, we put $h(\epsilon):=1-a^{4 \epsilon}\left(1-\log a^{4 \epsilon}\right)$. By taking the derivative with respect to $\epsilon$, we have

$$
h^{\prime}(\epsilon)=-a^{4 \epsilon} \log a^{4}\left(1-\log a^{4 \epsilon}\right)+a^{4 \epsilon} \log a^{4}=\epsilon a^{4 \epsilon}\left(\log a^{4}\right)^{2} \geq 0 .
$$

We also have $h(0)=0$. Hence, we obtain $h(\epsilon) \geq 0$.

To show the right inequality, we put as follows:

$$
\begin{aligned}
g(\epsilon): & =a^{-4 \epsilon}\left(3\left(a^{-\epsilon}-a^{\epsilon}\right)^{2} a^{\epsilon}-\left(1-a^{4 \epsilon}\left(1-\log a^{4 \epsilon}\right)\right)\right) \\
& =3\left(a^{-5 \epsilon}-2 a^{-3 \epsilon}+a^{-\epsilon}\right)+\left(1-\log a^{4 \epsilon}\right)-a^{-4 \epsilon}
\end{aligned}
$$

By taking the derivative with respect to $\epsilon$, we obtain the following:

$$
\begin{aligned}
g^{\prime}(\epsilon)= & 3\left(a^{-5 \epsilon}(-5 \log a)-2 a^{-3 \epsilon}(-3 \log a)+a^{-\epsilon}(-\log a)\right) \\
& -4 \log a-a^{-4 \epsilon}(-4 \log a), \\
g^{\prime \prime}(\epsilon)= & \left(75 a^{-5 \epsilon}-16 a^{-4 \epsilon}-54 a^{-3 \epsilon}+3 a^{-\epsilon}\right) \cdot(\log a)^{2}
\end{aligned}
$$

It is easy to check $g^{\prime \prime}(\epsilon) \geq 0$ by using $a^{-5 \epsilon} \geq a^{-k \epsilon}(k=3,4)$. Because $g^{\prime}(0)=$ $g(0)=0$, we obtain $g(\epsilon) \geq 0$. Thus we are done.

Lemma 4.6 Let $P(t)$ be a polynomial in a variable $t$, and let $b$ be any fixed positive number. Then we have the boundedness of $|z|^{b \epsilon} \cdot P\left(\epsilon L_{0}(z)\right)$ on $\Delta^{*}$, independently of $0 \leq \epsilon \leq 1 / 2$.

Proof We put $u:=|z|^{\epsilon}$, and then $|z|^{b \epsilon} P\left(\epsilon L_{0}(z)\right)=u^{b} \cdot P\left(L_{0}(u)\right)$. Hence, we have only to show the boundedness of $u^{b} \cdot P\left(L_{0}(u)\right)$ when $0<u<1$, which is easy. $\square$

\subsection{A family of metrics for a logarithmic $\lambda$-flat bundle of rank two on a disc}

4.3.1 Construction of a family of metrics We put $X:=\Delta=\{z|| z \mid<1\}$. Let $O$ denote the origin, and we put $X^{*}:=X-\{O\}$. We use the Kahler form $\omega_{\epsilon}:=$ $\left(\epsilon^{2}|z|^{\epsilon-2}+1\right) \cdot d z \cdot d \bar{z}$ in this subsection. We will use the notation in Section 4.2. 
To begin with, we recall an example of a harmonic bundle on a punctured disc. Let $E=\mathcal{O}_{X} \cdot v_{1} \oplus \mathcal{O}_{X} \cdot v_{2}$ be a holomorphic vector bundle on a disc. Let $\theta$ be a Higgs bundle such that $\theta \cdot v_{1}=v_{2} \cdot d z / z$ and $\theta \cdot v_{2}=0$. Let $h$ be the metric of $E_{\mid X^{*}}$ such that $h\left(v_{1}, v_{1}\right)=L_{0}, h\left(v_{2}, v_{2}\right)=L_{0}^{-1}$ and $h\left(v_{i}, v_{j}\right)=0(i \neq j)$. Recall that the tuple $\left(E, \bar{\partial}_{E}, \theta, h\right)$ is a harmonic bundle. Let us look at the associated $\lambda$-connection. We put $u_{1}:=v_{1}$ and $u_{2}:=v_{2}-\lambda \cdot L_{0}^{-1} \cdot v_{1}$. Then $\mathbf{u}=\left(u_{1}, u_{2}\right)$ gives a frame, and we can show $\left(\bar{\partial}_{E}+\lambda \theta^{\dagger}\right) u_{i}=0(i=1,2), \mathbb{D}^{\lambda} u_{1}=u_{2} \cdot d z / z$ and $\mathbb{D}^{\lambda} u_{2}=0$ by a direct calculation. We also have the following:

$h\left(u_{1}, u_{1}\right)=L_{0}, \quad h\left(u_{2}, u_{2}\right)=\left(1+|\lambda|^{2}\right) \cdot L_{0}^{-1}, \quad h\left(u_{1}, u_{2}\right)=-\bar{\lambda}, \quad h\left(u_{2}, u_{1}\right)=-\lambda$

Motivated by this example, we consider the following family of the metrics $h_{\epsilon}$ on the $\lambda$-connection $\left(E, \mathbb{D}^{\lambda}\right)$ given as follows:

$$
h_{\epsilon}\left(u_{1}, u_{1}\right)=L_{\epsilon}, \quad h_{\epsilon}\left(u_{2}, u_{2}\right)=\left(1+|\lambda|^{2}\right) \cdot L_{\epsilon}^{-1}, \quad h_{\epsilon}\left(u_{1}, u_{2}\right)=-\bar{\lambda} \cdot M_{\epsilon}
$$

The $\lambda$-connection $\mathbb{D}^{\lambda}$ and the metric $h_{\epsilon}$ induce the operators $\bar{\partial}_{\epsilon}$ and $\theta_{\epsilon}$ (Section 2.2.1). The main purpose of this subsection is to show the following proposition.

Proposition 4.7 There exists a some positive constant $C$ such that $\left|\bar{\partial}_{\epsilon} \theta_{\epsilon}\right|_{h_{\epsilon}, \omega_{\epsilon}} \leq C$ for any $0 \leq \epsilon<1 / 2$.

Although the proof of the proposition is just a calculation, we will give the details in the rest of this subsection.

Remark 4.8 Let $h_{\epsilon}^{\prime}$ be the metric determined by $h_{\epsilon}^{\prime}\left(u_{1}, u_{1}\right)=L_{\epsilon}, h_{\epsilon}^{\prime}\left(u_{2}, u_{2}\right)=L_{\epsilon}^{-1}$ and $h_{\epsilon}^{\prime}\left(u_{i}, u_{j}\right)=0(i \neq j)$. Then there exist positive constants $C_{i}(i=1,2)$ such that $C_{1} \cdot h_{\epsilon}^{\prime} \leq h_{\epsilon} \leq C_{2} \cdot h_{\epsilon}^{\prime}$ for any $0 \leq \epsilon \leq 1 / 2$. Hence, we have only to consider the norms for $h_{\epsilon}^{\prime}$ instead of those for $h_{\epsilon}$.

4.3.2 Preliminary Let $H_{\epsilon}$ be the hermitian matrix valued function given by $H_{\epsilon}:=$ $H\left(h_{\epsilon}, \mathbf{u}\right)$, ie,

$$
H_{\epsilon}:=\left(\begin{array}{cc}
L_{\epsilon} & -\bar{\lambda} \cdot M_{\epsilon} \\
-\lambda \cdot M_{\epsilon} & \left(1+|\lambda|^{2}\right) L_{\epsilon}^{-1}
\end{array}\right) .
$$


Let $N$ be determined by $\mathbb{D}^{\lambda} \mathbf{u}=\mathbf{u} \cdot N \cdot d z / z$, and let $N_{\epsilon}^{\dagger}$ denote the adjoint of $N$ with respect to the metric $H_{\epsilon}$, ie,

$$
\begin{aligned}
N & =\left(\begin{array}{ll}
0 & 0 \\
1 & 0
\end{array}\right), \\
N_{\epsilon}^{\dagger} & =\bar{H}_{\epsilon}^{-1 t} \bar{N} \bar{H}_{\epsilon} \\
& =\frac{1}{1+|\lambda|^{2}\left(1-M_{\epsilon}^{2}\right)}\left(\begin{array}{cc}
-\bar{\lambda}\left(1+|\lambda|^{2}\right) L_{\epsilon}^{-1} M_{\epsilon} & \left(1+|\lambda|^{2}\right)^{2} L_{\epsilon}^{-2} \\
-\bar{\lambda}^{2} M_{\epsilon}^{2} & \bar{\lambda}\left(1+|\lambda|^{2}\right) M_{\epsilon} L_{\epsilon}^{-1}
\end{array}\right) .
\end{aligned}
$$

Recall the calculation given in Section 2.2.2. Then $\bar{\partial}_{\epsilon}$ and $\theta_{\epsilon}$ can be described as follows:

$$
\bar{\partial}_{\epsilon} \mathbf{u}=\mathbf{u} \cdot \frac{\lambda}{1+|\lambda|^{2}}\left(\bar{\lambda} \cdot \bar{H}_{\epsilon}^{-1} \bar{\partial} \bar{H}_{\epsilon}-N_{\epsilon}^{\dagger} \frac{d \bar{z}}{\bar{z}}\right), \quad \theta_{\epsilon} \mathbf{u}=\mathbf{u} \frac{1}{1+|\lambda|^{2}}\left(N \frac{d z}{z}-\lambda \bar{H}_{\epsilon}^{-1} \partial \bar{H}_{\epsilon}\right)
$$

Therefore, $\bar{\partial}_{\epsilon}\left(\theta_{\epsilon}\right)$ is described by the following $2 \times 2$ matrix valued 2 -form with respect to $\mathbf{u}$ :

$$
\text { 39) } \begin{aligned}
& \frac{1}{1+|\lambda|^{2}} \bar{\partial}\left(-\lambda \bar{H}_{\epsilon}^{-1} \partial \bar{H}_{\epsilon}\right) \\
+ & \frac{\lambda}{\left(1+|\lambda|^{2}\right)^{2}}\left(\left[\bar{\lambda} \cdot \bar{H}_{\epsilon}^{-1} \bar{\partial} \bar{H}_{\epsilon}, N \frac{d z}{z}\right]-\left[N_{\epsilon}^{\dagger} \frac{d \bar{z}}{\bar{z}}, N \frac{d z}{z}\right]+\left[N_{\epsilon}^{\dagger} \frac{d \bar{z}}{\bar{z}}, \lambda \bar{H}_{\epsilon}^{-1} \partial \bar{H}_{\epsilon}\right]\right)
\end{aligned}
$$

Here we have used $\left[\bar{H}_{\epsilon}^{-1} \partial \bar{H}_{\epsilon}, \bar{H}_{\epsilon}^{-1} \bar{\partial} \bar{H}_{\epsilon}\right]=0$, which can be checked easily.

Lemma 4.9 To show Proposition 4.7, we have only to show the uniform boundedness of $(1,1)$-entry, $(2,2)$-entry, $L_{\epsilon} \times(1,2)$-entry and $L_{\epsilon}^{-1} \times(2,1)$-entry, in the matrix valued function (39).

Proof It follows from Remark 4.8.

In the following calculation, we often use the symbols $L$ and $M$ instead of $L_{\epsilon}$ and $M_{\epsilon}$, if there are no risk of confusion. Let us look at $\bar{H}_{\epsilon}^{-1} \partial \bar{H}_{\epsilon}$. We have the following equalities:

$$
\begin{aligned}
\bar{H}_{\epsilon}^{-1} & =\frac{1}{1+|\lambda|^{2}\left(1-M_{\epsilon}^{2}\right)}\left(\begin{array}{cc}
\left(1+|\lambda|^{2}\right) \cdot L_{\epsilon}^{-1} & \lambda \cdot M_{\epsilon} \\
\bar{\lambda} \cdot M_{\epsilon} & L_{\epsilon}
\end{array}\right), \\
\partial \bar{H}_{\epsilon} & =\left(\begin{array}{cc}
\partial L_{\epsilon} & -\lambda \cdot \partial M_{\epsilon} \\
-\bar{\lambda} \cdot \partial M_{\epsilon} & \left(1+|\lambda|^{2}\right) \cdot \partial L_{\epsilon}^{-1}
\end{array}\right)
\end{aligned}
$$


Then we obtain the following formula for $\bar{H}_{\epsilon}^{-1} \partial \bar{H}_{\epsilon}$ :

(40) $\bar{H}_{\epsilon}^{-1} \partial \bar{H}_{\epsilon}=\frac{1}{1+|\lambda|^{2}\left(1-M_{\epsilon}^{2}\right)} \times$

$$
\left(\begin{array}{cc}
\left(1+|\lambda|^{2}\right) L^{-1} \partial L-|\lambda|^{2} M \partial M & \lambda\left(1+|\lambda|^{2}\right)\left(-L^{-1} \partial M+M \partial L^{-1}\right) \\
\bar{\lambda}(M \partial L-L \partial M) & \left(1+|\lambda|^{2}\right) L \partial L^{-1}-|\lambda|^{2} M \cdot \partial M
\end{array}\right)
$$

We also have a similar formula for $\bar{H}_{\epsilon}^{-1} \bar{\partial} \bar{H}_{\epsilon}$. We obtain the following formula for $\bar{\partial}\left(\bar{H}_{\epsilon}^{-1} \partial \bar{H}_{\epsilon}\right)$ :

$$
\begin{aligned}
& \bar{\partial}\left(\bar{H}_{\epsilon}^{-1} \partial \bar{H}_{\epsilon}\right)=\frac{2|\lambda|^{2} M \bar{\partial} M}{\left(1+|\lambda|^{2}\left(1-M^{2}\right)\right)^{2}} \bar{H}_{\epsilon}^{-1} \partial \bar{H}_{\epsilon}+\frac{1}{1+|\lambda|^{2}\left(1-M^{2}\right)} \times \\
& \left(\begin{array}{cc}
\left(1+|\lambda|^{2}\right) \bar{\partial} \partial \log L-2^{-1}|\lambda|^{2} \bar{\partial} \partial M^{2} & \lambda\left(1+|\lambda|^{2}\right)\left(M \bar{\partial} \partial L^{-1}-L^{-1} \bar{\partial} \partial M\right) \\
\bar{\lambda}(M \bar{\partial} \partial L-L \bar{\partial} \partial M) & \left(1+|\lambda|^{2}\right) \bar{\partial} \partial \log L^{-1}-2^{-1}|\lambda|^{2} \bar{\partial} \partial M^{2}
\end{array}\right)
\end{aligned}
$$

The commutator of $\bar{H}_{\epsilon}^{-1} \bar{\partial} \bar{H}_{\epsilon}$ and $N \cdot d z / z$ is as follows:

$$
\begin{aligned}
& {\left[\bar{H}_{\epsilon}^{-1} \bar{\partial} \bar{H}_{\epsilon}, N \cdot \frac{d z}{z}\right]=\frac{\left(1+|\lambda|^{2}\right)}{1+|\lambda|^{2}\left(1-M^{2}\right)} \times } \\
&\left(\begin{array}{cc}
\lambda\left(-L^{-1} \bar{\partial} M+M \bar{\partial} L^{-1}\right) & 0 \\
2 L \bar{\partial} L^{-1} & -\lambda\left(-L^{-1} \bar{\partial} M+M \bar{\partial} L^{-1}\right)
\end{array}\right) \frac{d z}{z}
\end{aligned}
$$

Let us look at the commutator of $\bar{H}_{\epsilon}^{-1} \partial \bar{H}_{\epsilon}$ and $N_{\epsilon}^{\dagger}$. By direct calculations, we have the following equality:

$$
\begin{aligned}
& \bar{H}_{\epsilon}^{-1} \partial \bar{H}_{\epsilon} \cdot N_{\epsilon}^{\dagger} \\
&=\frac{1}{1+|\lambda|^{2}\left(1-M^{2}\right)}\left(\begin{array}{cc}
-\bar{\lambda}\left(1+|\lambda|^{2}\right) L^{-2} M \partial L & \left(1+|\lambda|^{2}\right)^{2} L^{-3} \partial L \\
\bar{\lambda}^{2} \cdot M \partial M & -\bar{\lambda}\left(1+|\lambda|^{2}\right) L^{-1} \partial M
\end{array}\right) \\
& \quad+\frac{1}{\left(1+|\lambda|^{2}\left(1-M^{2}\right)\right)^{2}} \\
& \quad \times\left(\begin{array}{cc}
2|\lambda|^{2} \bar{\lambda}\left(1+|\lambda|^{2}\right) M^{2} L^{-1} \partial M & -2|\lambda|^{2}\left(1+|\lambda|^{2}\right)^{2} M L^{-2} \partial M \\
2 M^{3} \partial M \bar{\lambda}^{2}|\lambda|^{2} & -2 \bar{\lambda}|\lambda|^{2}\left(1+|\lambda|^{2}\right) M^{2} L^{-1} \partial M
\end{array}\right)
\end{aligned}
$$

We also have the following:

$N_{\epsilon}^{\dagger} \cdot \bar{H}_{\epsilon}^{-1} \partial \bar{H}_{\epsilon}$

$$
=\frac{1}{1+|\lambda|^{2}\left(1-M^{2}\right)}\left(\begin{array}{cc}
-\bar{\lambda}\left(1+|\lambda|^{2}\right) L^{-1} \partial M & \left(1+|\lambda|^{2}\right)^{2} L^{-1} \partial L^{-1} \\
-\bar{\lambda}^{2} M \partial M & \bar{\lambda}\left(1+|\lambda|^{2}\right) M \partial L^{-1}
\end{array}\right)
$$


Therefore, we obtain the following formula:

$$
\begin{aligned}
& \left(1+|\lambda|^{2}\left(1-M^{2}\right)\right)\left[N_{\epsilon}^{\dagger}, \bar{H}_{\epsilon}^{-1} \partial \bar{H}_{\epsilon}\right] \\
= & \left(\begin{array}{cc}
-\bar{\lambda}\left(1+|\lambda|^{2}\right)\left(L^{-1} \partial M-L^{-2} M \partial L\right) & -2\left(1+|\lambda|^{2}\right)^{2} L^{-3} \partial L \\
-2 \bar{\lambda}^{2} M \partial M & \bar{\lambda}\left(1+|\lambda|^{2}\right)\left(M \partial L^{-1}+L^{-1} \partial M\right)
\end{array}\right) \\
& -\frac{2|\lambda|^{2}}{\left(1+|\lambda|^{2}\left(1-M^{2}\right)\right)}\left(\begin{array}{cc}
\bar{\lambda}\left(1+|\lambda|^{2}\right) M^{2} L^{-1} \partial M & -\left(1+|\lambda|^{2}\right)^{2} M L^{-2} \partial M \\
\overline{\lambda^{2}} M^{3} \partial M & -\bar{\lambda}\left(1+|\lambda|^{2}\right) M^{2} L^{-1} \partial M
\end{array}\right)
\end{aligned}
$$

The commutator of $N$ and $N_{\epsilon}^{\dagger}$ is as follows:

$$
\left[N_{\epsilon}^{\dagger}, N\right]=\frac{1}{1+|\lambda|^{2}\left(1-M^{2}\right)}\left(\begin{array}{cc}
\left(1+|\lambda|^{2}\right)^{2} L^{-2} & 0 \\
2 \bar{\lambda}\left(1+|\lambda|^{2}\right) M L^{-1} & -\left(1+|\lambda|^{2}\right)^{2} L^{-2}
\end{array}\right)
$$

4.3.3 Estimate We have the following:

$$
\partial L_{\epsilon}=-K_{\epsilon} \frac{d z}{z}, \quad \partial K_{\epsilon}=-\frac{\epsilon^{2}}{4} L_{\epsilon} \frac{d z}{z}, \quad \partial M_{\epsilon}=4 \epsilon^{2} \cdot|z|^{4 \epsilon} \cdot L_{0} \cdot \frac{d z}{z}
$$

In particular, we have the following estimate:

$$
M_{\epsilon} \partial M_{\epsilon}=O\left(\epsilon^{2} \cdot|z|^{8 \epsilon} \cdot L_{0} \cdot\left(1+\epsilon L_{0}\right) \frac{d z}{z}\right)
$$

Let us look at the first term in the right hand side of (41):

$$
\frac{2|\lambda|^{2} M_{\epsilon} \bar{\partial} M_{\epsilon}}{\left(1+|\lambda|^{2}\left(1-M_{\epsilon}^{2}\right)\right)^{2}} \bar{H}_{\epsilon}^{-1} \partial \bar{H}_{\epsilon}
$$

For the $(1,1)$-entry and $(2,2)$-entry, we have the following estimates:

$$
\begin{aligned}
M_{\epsilon} \bar{\partial} M_{\epsilon} L_{\epsilon}^{-1} \partial L_{\epsilon} & =O\left(\epsilon^{2} L_{0}|z|^{8 \epsilon}\left(1+\epsilon L_{0}\right) \frac{K_{\epsilon}}{L_{\epsilon}}\right) \frac{d \bar{z} d z}{|z|^{2}} \\
& =O\left(|z|^{5 \epsilon}\left(1+\epsilon L_{0}\right) \frac{L_{0}}{L_{\epsilon}}\right) \omega_{\epsilon} \\
M_{\epsilon} \bar{\partial} M_{\epsilon} M_{\epsilon} \partial M_{\epsilon} & =O\left(\epsilon^{4}|z|^{16 \epsilon}\left(1+\epsilon L_{0}\right)^{2} L_{0}^{2}\right) \frac{d z d \bar{z}}{|z|^{2}} \\
& =O\left(|z|^{15 \epsilon}\left(1+\epsilon L_{0}\right)^{2}\left(\epsilon L_{0}\right)^{2}\right) \omega_{\epsilon}
\end{aligned}
$$

They are bounded with respect to $\omega_{\epsilon}$ due to Lemma 4.3 and Lemma 4.6. Hence, the $(1,1)$-entry and the $(2,2)$-entry of $(45)$ are bounded independently of $\epsilon$. Let us look at the $(1,2)$-entry. Recall Lemma 4.9. Hence, we have only to see the following:

$$
L_{\epsilon} \times\left(M_{\epsilon} \bar{\partial} M_{\epsilon}\right) \cdot\left(L_{\epsilon}^{-1} \partial M_{\epsilon}-M_{\epsilon} \partial L_{\epsilon}^{-1}\right)=M_{\epsilon} \bar{\partial} M_{\epsilon} \partial M_{\epsilon}+M_{\epsilon}^{2} \bar{\partial} M_{\epsilon} L_{\epsilon}^{-1} \partial L_{\epsilon}
$$


Both terms in the right hand side can be estimated as in the previous case, by using Lemma 4.3 and Lemma 4.6:

$$
\begin{aligned}
M_{\epsilon} \bar{\partial} M_{\epsilon} \partial M_{\epsilon} & =O\left(|z|^{10 \epsilon}\left(1+\epsilon L_{0}\right)\left(\epsilon L_{0}\right)^{2}\right) \cdot \omega_{\epsilon}=O(1) \cdot \omega_{\epsilon} \\
M_{\epsilon}{ }_{\epsilon} \bar{\partial} M_{\epsilon} L_{\epsilon}^{-1} \partial L_{\epsilon} & =O\left(|z|^{11 \epsilon}\left(1+\epsilon L_{0}\right)^{2} \frac{L_{0}}{L_{\epsilon}}\right) \cdot \omega_{\epsilon}=O(1) \cdot \omega_{\epsilon}
\end{aligned}
$$

The $(2,1)$-entry can be estimated similarly:

$L_{\epsilon}^{-1} \times\left(M_{\epsilon} \bar{\partial} M_{\epsilon}\right)\left(M_{\epsilon} \partial L_{\epsilon}-L_{\epsilon} \partial M_{\epsilon}\right)=M_{\epsilon}^{2} L_{\epsilon}^{-1} \bar{\partial} M_{\epsilon} \partial L_{\epsilon}-M_{\epsilon} \cdot \bar{\partial} M_{\epsilon} \partial M_{\epsilon}=O(1) \cdot \omega_{\epsilon}$

Let us look at the second term in the right hand side of (41), which is the product of $\left(1+|\lambda|^{2}\left(1-M^{2}\right)\right)^{-1}$ and the following:

$$
\left(\begin{array}{cc}
\left(1+|\lambda|^{2}\right) \bar{\partial} \partial \log L-2^{-1}|\lambda|^{2} \bar{\partial} \partial M^{2} & \lambda\left(1+|\lambda|^{2}\right)\left(M \bar{\partial} \partial L^{-1}-L^{-1} \bar{\partial} \partial M\right) \\
\bar{\lambda}\left(1+|\lambda|^{2}\right)(M \bar{\partial} \partial L-L \bar{\partial} \partial M) & \left(1+|\lambda|^{2}\right) \bar{\partial} \partial \log L^{-1}-2^{-1}|\lambda|^{2} \bar{\partial} \partial M^{2}
\end{array}\right)
$$

It is easy to see the following estimate:

$$
\bar{\partial} \partial M_{\epsilon}^{2}=O\left(\epsilon^{2} \cdot|z|^{6 \epsilon}\left(1+\epsilon L_{0}\right)^{2}\right) \cdot \omega_{\epsilon}=O\left(\epsilon^{2}\right) \cdot \omega_{\epsilon}
$$

So it is bounded with respect to $\omega_{\epsilon}$ independently of $\epsilon$. We remark that $L_{\epsilon}^{-1} M_{\epsilon} \bar{\partial} \partial L_{\epsilon}$ is also bounded independently of $\epsilon$ :

$$
L_{\epsilon}^{-1} M_{\epsilon} \cdot \bar{\partial} \partial L_{\epsilon}=\frac{\epsilon^{2}}{4} M_{\epsilon} \cdot \frac{d \bar{z} \cdot d z}{|z|^{2}}=O(1) \cdot \omega_{\epsilon}
$$

Hence, we have the following, modulo the uniformly bounded term with respect to $\left(h_{\epsilon}, \omega_{\epsilon}\right)$ :

$$
\bar{\partial}\left(\bar{H}_{\epsilon}^{-1} \partial \bar{H}_{\epsilon}\right) \equiv \frac{\left(1+|\lambda|^{2}\right)}{1+|\lambda|^{2}\left(1-M_{\epsilon}^{2}\right)}\left(\begin{array}{cc}
\bar{\partial} \partial \log L_{\epsilon} & \lambda M_{\epsilon} \bar{\partial} \partial L_{\epsilon}^{-1} \\
0 & -\bar{\partial} \partial \log L_{\epsilon}
\end{array}\right)
$$

Let us look at (42). By the same argument, we have the following uniform boundedness:

$$
L_{\epsilon}^{-1} \bar{\partial} M_{\epsilon} \cdot \frac{d z}{z}=O\left(\epsilon^{2}|z|^{4 \epsilon} \frac{L_{0}}{L_{\epsilon}}\right) \cdot \frac{d z \cdot d \bar{z}}{|z|^{2}}=O(1) \cdot \omega_{\epsilon}
$$

Hence, we have the following, modulo the uniformly bounded terms with respect to $\left(h_{\epsilon}, \omega_{\epsilon}\right)$ :

$$
\left[\bar{H}_{\epsilon}^{-1} \bar{\partial} \bar{H}_{\epsilon}, \quad N \cdot \frac{d z}{z}\right] \equiv \frac{\left(1+|\lambda|^{2}\right)}{1+|\lambda|^{2}\left(1-M_{\epsilon}^{2}\right)}\left(\begin{array}{cc}
\lambda M_{\epsilon} \bar{\partial} L_{\epsilon}^{-1} & 0 \\
2 L_{\epsilon} \bar{\partial} L_{\epsilon}^{-1} & -\lambda M_{\epsilon} \bar{\partial} L_{\epsilon}^{-1}
\end{array}\right) \cdot \frac{d z}{z}
$$


Let us look at (43). We remark the following, for any $k \geq 1$ :

$$
\frac{d \bar{z}}{\bar{z}} \frac{M_{\epsilon}^{k} \partial M_{\epsilon}}{L_{\epsilon}}=O\left(\epsilon^{2}|z|^{4(k+1) \epsilon}\left(1+\epsilon L_{0}\right)^{k} \frac{L_{0}}{L_{\epsilon}}\right) \cdot \frac{d \bar{z} \cdot d z}{|z|^{2}}=O(1) \cdot \omega_{\epsilon}
$$

Hence, the terms containing $\partial M$ in the right hand side of (43) can be ignored. Hence, we obtain the following, modulo the uniformly bounded terms with respect to $\left(h_{\epsilon}, \omega_{\epsilon}\right)$ :

$$
\begin{aligned}
& {\left[N_{\epsilon}^{\dagger} \frac{d \bar{z}}{\bar{z}}, \bar{H}_{\epsilon}^{-1} \partial \bar{H}_{\epsilon}\right]} \\
& \quad \equiv \frac{\left(1+|\lambda|^{2}\right)}{1+|\lambda|^{2}\left(1-M_{\epsilon}^{2}\right)} \frac{d \bar{z}}{\bar{z}}\left(\begin{array}{cc}
\bar{\lambda} L_{\epsilon}^{-2} M_{\epsilon} \partial L_{\epsilon} & -2\left(1+|\lambda|^{2}\right) L_{\epsilon}^{-3} \partial L_{\epsilon} \\
0 & \bar{\lambda} M_{\epsilon} \partial L_{\epsilon}^{-1}
\end{array}\right)
\end{aligned}
$$

In all, (39) is equal to the following, modulo uniformly bounded terms due to (44), (47), (48) and (49):

$$
\frac{1}{1+|\lambda|^{2}\left(1-M_{\epsilon}^{2}\right)}\left(\begin{array}{cc}
-\lambda \bar{\partial} \partial \log L_{\epsilon} & -\lambda^{2} M_{\epsilon} \cdot \bar{\partial} \partial L_{\epsilon}^{-1} \\
0 & \lambda \bar{\partial} \partial \log L_{\epsilon}
\end{array}\right)
$$

$$
\begin{aligned}
& +\frac{1}{1+|\lambda|^{2}\left(1-M_{\epsilon}^{2}\right)} \frac{|\lambda|^{2}}{1+|\lambda|^{2}} \frac{d \bar{z} \cdot d z}{|z|^{2}}\left(\begin{array}{cc}
\lambda \cdot M_{\epsilon} \cdot K_{\epsilon} \cdot L_{\epsilon}^{-2} & 0 \\
2 K_{\epsilon} \cdot L_{\epsilon}^{-1} & -\lambda \cdot M_{\epsilon} \cdot K_{\epsilon} \cdot L_{\epsilon}^{-2}
\end{array}\right) \\
& +\frac{1}{1+|\lambda|^{2}\left(1-M_{\epsilon}^{2}\right)} \frac{\lambda^{2}}{1+|\lambda|^{2}} \frac{d \bar{z} \cdot d z}{|z|^{2}}\left(\begin{array}{cc}
-\bar{\lambda} \cdot M_{\epsilon} \cdot K_{\epsilon} \cdot L_{\epsilon}^{-2} & 2\left(1+|\lambda|^{2}\right) L_{\epsilon}^{-3} \cdot K_{\epsilon} \\
0 & \bar{\lambda} \cdot M_{\epsilon} \cdot K_{\epsilon} \cdot L_{\epsilon}^{-2}
\end{array}\right)
\end{aligned}
$$$$
-\frac{\lambda}{1+|\lambda|^{2}\left(1-M_{\epsilon}^{2}\right)} \frac{d \bar{z} \cdot d z}{|z|^{2}}\left(\begin{array}{cc}
L_{\epsilon}^{-2} & 0 \\
2 \bar{\lambda}\left(1+|\lambda|^{2}\right)^{-1} M_{\epsilon} \cdot L_{\epsilon}^{-1} & -L_{\epsilon}^{-2}
\end{array}\right)
$$

The summation of the last three term in (50) is as follows:

$$
\frac{1}{1+|\lambda|^{2}\left(1-M_{\epsilon}^{2}\right)} \frac{d \bar{z} \cdot d z}{|z|^{2}}\left(\begin{array}{cc}
-\lambda L_{\epsilon}^{-2} & 2 \lambda^{2} L_{\epsilon}^{-3} K_{\epsilon} \\
2|\lambda|^{2}\left(1+|\lambda|^{2}\right)^{-1}\left(K_{\epsilon}-M_{\epsilon}\right) L_{\epsilon}^{-1} & \lambda L_{\epsilon}^{-2}
\end{array}\right)
$$

By a direct calculation, we can show the following equalities:

$$
\bar{\partial} \partial \log L_{\epsilon}=-\frac{1}{L_{\epsilon}^{2}} \frac{d \bar{z} \cdot d z}{|z|^{2}}, \quad \bar{\partial} \partial L_{\epsilon}^{-1}=\frac{2}{L_{\epsilon}^{3}} \frac{d \bar{z} \cdot d z}{|z|^{2}}-\frac{\epsilon^{2}}{2} \frac{1}{L_{\epsilon}} \frac{d \bar{z} \cdot d z}{|z|^{2}}
$$


Therefore, (50) can be rewritten as follows:

$$
\begin{aligned}
& \frac{1}{1+|\lambda|^{2}\left(1-M_{\epsilon}^{2}\right)} \\
& \times\left(\begin{array}{cc}
2|\lambda|^{2}\left(1+|\lambda|^{2}\right)^{-1} L_{\epsilon}^{-1}\left(K_{\epsilon}-M_{\epsilon}\right) & 2 \lambda^{2} L_{\epsilon}^{-3}\left(K_{\epsilon}-M_{\epsilon}\right) \\
0
\end{array}\right) \frac{d \bar{z} d z}{|z|^{2}} \\
& \quad+\frac{1}{1+|\lambda|^{2}\left(1-M_{\epsilon}^{2}\right)}\left(\begin{array}{cc}
0 & \lambda^{2} \epsilon^{2} M_{\epsilon}\left(2 L_{\epsilon}\right)^{-1} \\
0 & 0
\end{array}\right) \frac{d \bar{z} d z}{|z|^{2}}
\end{aligned}
$$

Due to $M_{\epsilon}=O\left(|z|^{4 \epsilon}\left(1+\epsilon L_{0}\right)\right)$, the second term in (51) can be ignored. Due to Lemma 4.5 and Lemma 4.4, we have the uniform boundedness of $\left(M_{\epsilon}-1\right) \cdot L_{\epsilon}^{-2} \cdot d z \cdot d \bar{z} /|z|^{2}$ and $\left(K_{\epsilon}-1\right) \cdot L_{\epsilon}^{-2} \cdot d z \cdot d \bar{z} /|z|^{2}$. Thus, the proof of Proposition 4.7 is finished.

\subsection{A family of metrics of a parabolic $\lambda$-flat bundle on a disc}

4.4.1 Simple case We put $X:=\Delta=\{z \in \mathbf{C}|| z \mid<1\}$ and $X^{*}:=\Delta-\{O\}$. Let $V_{l}$ be a vector space over $\mathbf{C}$ with a base $\mathbf{e}=\left(e_{1}, \ldots, e_{l}\right)$, and let $N_{l}$ be the nilpotent endomorphism of $V_{l}$ given by $N_{l} \cdot e_{i+1}=e_{i}$ for $i=1, \ldots, l-1$ and $N_{l} \cdot e_{l}=0$. We put $E_{l}:=\mathcal{O}_{X} \otimes V_{l}$. Then $e_{i}$ naturally induce the frame of $E_{l}$, which we denote by $\mathbf{v}=\left(v_{1}, \ldots, v_{l}\right)$. The fiber $E_{\mid O}$ is naturally identified with $V$, and we have $\mathbf{v}_{\mid O}=\mathbf{e}$. We have the logarithmic $\lambda$-connection $\mathbb{D}_{l}^{\lambda}$ of $E_{l}$ given by $\mathbb{D}_{l}^{\lambda} v_{i}=v_{i+1} \cdot d z / z$ for $i=1, \ldots, l-1$ and $\mathbb{D}_{l}^{\lambda} v_{l}=0$. The residue $\operatorname{Res}\left(\mathbb{D}^{\lambda}\right)$ is given by $N_{l}$. We have the weight filtration $W$ of $E_{\mid O}$ with respect to $N_{l}$.

We have the trivial parabolic structure $F$ of $E_{l}$. Take a sufficiently small positive number $\epsilon$. We consider the $\epsilon$-perturbation $F^{(\epsilon)}$ given by $F_{k \epsilon}^{(\epsilon)}=W_{k}$ for $k=-l+$ $1,-l+3 \ldots, l-1$ in this case.

Let us fix a sufficiently small positive number $\epsilon_{0}$ such that $\operatorname{rank} E \cdot \epsilon_{0}<\eta / 10$. In the previous subsection, we have constructed a family of metrics $h_{2}^{(\epsilon)}\left(0 \leq \epsilon \leq \epsilon_{0}\right)$. It induces a metric of $\operatorname{Sym}^{l-1}\left(E_{2}, \mathbb{D}_{2}^{\lambda}\right) \simeq\left(E_{l}, \mathbb{D}_{l}\right)$, which we denote by $h_{l}^{(\epsilon)}$. The following property can be shown by reducing to the case $l=2$.

- $h_{l}^{(0)}$ is a harmonic metric.

- $h_{l}^{(\epsilon)} \longrightarrow h_{l}^{(0)}$ as $\epsilon \rightarrow 0$ in the $C^{\infty}$-sense locally on $X^{*}$.

- There exists a constant $C>0$ such that $\left|\Lambda_{\omega_{\epsilon}} G\left(h_{l}^{(\epsilon)}\right)\right|_{h_{l}^{(\epsilon)}}<C$.

- $h_{l}^{(\epsilon)}$ is adapted to the parabolic structure $F^{(\epsilon)}$.

- We set $t_{\epsilon}:=\operatorname{det}\left(h_{l}^{(\epsilon)}\right) / \operatorname{det}\left(h_{l}^{(0)}\right)$. Then $t_{\epsilon}$ and $t_{\epsilon}^{-1}$ are bounded, independently of $\epsilon$. 
Lemma 4.10 Let $H_{\epsilon}$ denote the Hermitian matrix valued function whose $(i, j)-$ entries are given by $\left(h_{l}^{(\epsilon)}\left(v_{i}, v_{j}\right)\right)$. Then we have the following estimate on $\{0<|z|<$ $1 / 2\}$ with respect to $h_{l}^{(\epsilon)}$ :

$$
\bar{H}_{\epsilon}^{-1} \cdot(\bar{\partial}+\lambda \partial) \bar{H}_{\epsilon}=O(1) \cdot \frac{d z}{z}+O(1) \cdot \frac{d \bar{z}}{\bar{z}}
$$

Proof We show the estimate only for $\bar{H}_{\epsilon}^{-1} \partial \bar{H}_{\epsilon}$. The term $\bar{H}_{\epsilon}^{-1} \bar{\partial} \bar{H}_{\epsilon}$ can be shown in the same way. We have only to check the case $l=2$. As in Lemma 4.9, we have only to look at the $(1,1)$-entry, $(2,2)$-entry, $L_{\epsilon} \times(1,2)$-entry and $L_{\epsilon}^{-1} \times(2,1)$-entry in the matrix valued function (40). As is seen in Section 4.3.3, the term containing $\partial M_{\epsilon}$ is bounded with respect to $\omega_{\epsilon}$, and the estimate is uniform for $\epsilon$. Hence, we can ignore them. Therefore, we have only to show that $L_{\epsilon}^{-1} \partial L_{\epsilon}=-L_{\epsilon} \partial L_{\epsilon}^{-1}$ is $O(1) \cdot d z / z$, but it can be checked by a direct calculation.

4.4.2 General case Let $\left(E, \mathbf{F}, \mathbb{D}^{\lambda}\right)$ be a parabolic flat $\lambda$-connection on $(X, O)$. Take a positive number $\eta$ such that $10 \cdot \eta<\operatorname{gap}(E, \mathbf{F})$. We will use the metrics

$$
\omega_{\epsilon}=\epsilon^{2}|z|^{\epsilon} \frac{d z \cdot d \bar{z}}{|z|^{2}}+|z|^{2 \eta} \frac{d z \cdot d \bar{z}}{|z|^{2}} .
$$

Here, $\epsilon$ will be $m^{-1}$ for some $m \in \mathbf{Z}_{>0}$ such that $10 \operatorname{rank}(E) \cdot \epsilon<\eta$. We take the $\epsilon$-perturbation $\mathbf{F}^{(\epsilon)}$ as in (II) of Section 2.1.6. Let $a(\epsilon)$ be the numbers which are denoted by $a(\epsilon, i)$ in the explanation there.

We have the endomorphism $\operatorname{Res}\left(\mathbb{D}^{\lambda}\right)$ of $\mathrm{Gr}_{a}^{F}(E)$. It induces the generalized eigen decomposition $\operatorname{Gr}_{a}^{F}(E)=\bigoplus_{\alpha \in \mathbf{C}} \operatorname{Gr}_{a, \alpha}^{F, \mathbb{E}}(E)$. On $\operatorname{Gr}_{u}^{F, \mathbb{E}}(E)$, the endomorphism Res $\left(\mathbb{D}^{\lambda}\right)$ is decomposed as $\alpha \cdot \mathrm{id}+N_{u}$, where $u=(a, \alpha) \in \mathbf{R} \times \mathbf{C}$. Let $W$ be the weight filtration of $N_{u}$ on $\mathrm{Gr}_{u}^{F, \mathbb{E}}(E)$. They induce the filtration $W$ of $\mathrm{Gr}_{a}^{F}(E)$.

For $u \in \mathbf{R} \times \mathbf{C}$, we put $V_{u}:=\mathrm{Gr}_{u}^{F, \mathbb{E}}(E)$ with the induced nilpotent map $N_{u}$. Then we can take an isomorphism

$$
\left(V_{u}, N_{u}\right) \simeq \bigoplus_{i=1}^{m(u)}\left(V_{l(u, i)}, N_{l(u, i)}\right),
$$

where $\left(V_{l}, N_{l}\right)$ are as in Section 4.4.1. We put

$$
\left(E_{u}, \mathbb{D}_{u}^{\lambda}\right):=\bigoplus\left(E_{l(u, i)}, \mathbb{D}_{l(u, i)}^{\lambda}\right)
$$

Let $h_{u}^{\prime(\epsilon)}$ denote the metric of $E_{u}$ induced by $h_{l(u, i)}^{(\epsilon)}(i=1, \ldots, m(u))$. (See Section 4.4.1). 
Let $Q(u)$ denote the logarithmic $\lambda$-flat bundle of rank one for $u=(a, \alpha)$, which is given by $\mathcal{O}_{X} \cdot e$ with the $\lambda$-connection $\mathbb{D}^{\lambda} e=e \cdot \alpha \cdot d z / z$. It is equipped with the family of the harmonic metrics $h_{u, \epsilon}^{\prime \prime(\epsilon)}(e, e)=|z|^{-2 a(\epsilon)}$. Then we obtain the vector bundle $E_{0}$ with the $\lambda$-connection $\mathbb{D}_{0}^{\lambda}$ and the parabolic structure $F$, as follows:

$$
\left(E_{0}, \mathbb{D}_{0}^{\lambda}\right)=\bigoplus_{u}\left(E_{u}, \mathbb{D}_{u}^{\lambda}\right) \otimes Q(u), \quad F_{b}\left(E_{0 \mid O}\right)=\bigoplus_{a \leq b} E_{(a, \alpha) \mid O} \otimes Q(a, \alpha)_{\mid O} .
$$

The metrics $h_{u}^{\prime(\epsilon)}$ and $h_{u}^{\prime \prime(\epsilon)}$ induce a metric $h_{u}^{(\epsilon)}$ of $E_{u} \otimes Q(u)$. Let $h_{0}^{(\epsilon)}$ denote the direct sum of them. We can take a holomorphic isomorphism $\Psi: E_{0} \longrightarrow E$ satisfying the following conditions:

- It preserves the filtration $F$.

- $\mathrm{Gr}^{F}(\Psi) \circ \mathrm{Gr}^{F} \operatorname{Res} \mathbb{D}^{\lambda}=\mathrm{Gr}^{F} \operatorname{Res} \mathbb{D}_{0}^{\lambda} \circ \mathrm{Gr}^{F}(\Psi)$.

We identify $E_{0}$ and $E$ via $\Psi$. The naturally induced metric of $E$ is denoted by the same symbol $h_{0}^{(\epsilon)}$.

Lemma 4.11 The family of the hermitian metrics $\left\{h_{0}^{(\epsilon)} \mid 0 \leq \epsilon \leq \epsilon_{0}\right\}$ has the following properties:

- $G\left(\mathbb{D}^{\lambda}, h_{0}^{(\epsilon)}\right)$ is uniformly bounded with respect to $\left(\omega_{\epsilon}, h_{0}^{(\epsilon)}\right)$.

- $\left\{h_{0}^{(\epsilon)} \mid \epsilon>0\right\}$ converges to $h_{0}^{(0)}$ in the $C^{\infty}$-sense locally on $X^{*}$.

- $h_{0}^{(\epsilon)}$ is adapted to the perturbed parabolic structure $F^{(\epsilon)}$.

- Let $t_{\epsilon}$ be determined by $\operatorname{det}\left(h_{0}^{(\epsilon)}\right) / \operatorname{det}\left(h_{0}^{(0)}\right)$. Then $t_{\epsilon}$ and $t_{\epsilon}^{-1}$ are bounded, independently from $\epsilon$.

Proof We check only the first claim. The other claims are easy to see. Let $f$ be determined by $f \cdot d z / z=\mathbb{D}^{\lambda}-\mathbb{D}_{0}^{\lambda}$, and we put $f_{\epsilon}^{\dagger}:=f_{h^{(\epsilon)}}^{\dagger}$. We put $\mathbb{D}_{\epsilon}^{\lambda \star}:=\mathbb{D}_{h^{(\epsilon)}}^{\lambda \star}$ and $\mathbb{D}_{0, \epsilon}^{\lambda \star}:=\mathbb{D}_{0, h^{(\epsilon)}}^{\lambda \star}$. Then we have the following:

$$
\begin{aligned}
G\left(\mathbb{D}^{\lambda}, h_{0}^{(\epsilon)}\right) & =\left[\mathbb{D}^{\lambda}, \mathbb{D}_{\epsilon}^{\lambda \star}\right]=\left[\mathbb{D}_{0}^{\lambda}+f \frac{d z}{z}, \mathbb{D}_{0, \epsilon}^{\lambda \star}+f_{\epsilon}^{\dagger} \frac{d \bar{z}}{\bar{z}}\right] \\
& =G\left(\mathbb{D}_{0}^{\lambda}, h_{0}^{(\epsilon)}\right)+\mathbb{D}_{0, \epsilon}^{\lambda \star}(f) \frac{d z}{z}+\mathbb{D}_{0}^{\lambda}\left(f_{\epsilon}^{\dagger}\right) \frac{d \bar{z}}{\bar{z}}+\left[f, f_{\epsilon}^{\dagger}\right] \frac{d z \cdot d \bar{z}}{|z|^{2}}
\end{aligned}
$$

We have the decomposition $f=\sum f_{u, u^{\prime}}$, where $f_{u, u^{\prime}} \in \operatorname{Hom}\left(E_{u} \otimes Q(u), E_{u^{\prime}} \otimes Q\left(u^{\prime}\right)\right)$. We have $f_{u, u^{\prime} \mid O}=0$ unless $\alpha=\alpha^{\prime}$ and $a>a^{\prime}$. Hence, there exist positive constants $C$ and $N$ such that the following holds for $0<\epsilon<\epsilon_{0}$ :

$$
|f|_{h_{0}^{(\epsilon)}} \leq C \cdot|z|^{10 \eta} L_{\epsilon}^{N}
$$


Here $N \cdot \epsilon$ is sufficiently smaller than $\eta$. Hence, we have the following:

$$
|f|_{h_{0}^{(\epsilon)}} \leq C \cdot|z|^{9 \eta}, \quad\left|\left[f, f_{\epsilon}^{\dagger}\right]\right|_{h^{(\epsilon)}} \leq C \cdot|z|^{18 \eta}
$$

We have the induced frames $\mathbf{v}_{u}$ of $E_{u} \otimes Q(u)$. They induce the frame $\mathbf{v}$ of $E_{0}$. Let $B$ and $A_{0}$ be determined by $f \mathbf{v}=\mathbf{v} \cdot B \cdot d z / z$ and $\mathbb{D}_{0}^{\lambda} \mathbf{v}=\mathbf{v} \cdot A_{0} \cdot d z / z$. Then we have the following:

$$
\left[\mathbb{D}_{0}^{\lambda}, f^{\dagger}\right] \mathbf{v}=\mathbf{v}\left(\mathcal{D} B_{\epsilon}^{\dagger} \frac{d \bar{z}}{\bar{z}}+\left[A_{0}, B_{\epsilon}^{\dagger}\right] \frac{d z \cdot d \bar{z}}{|z|^{2}}\right)
$$

Here we put $\mathcal{D}=\bar{\partial}+\lambda \partial$ and $B_{\epsilon}^{\dagger}=\bar{H}_{\epsilon}^{-1} \cdot{ }^{t} \bar{B} \cdot \bar{H}_{\epsilon}$, where $H_{\epsilon}=H\left(h_{0}^{(\epsilon)}, \mathbf{u}\right)$. Since $B_{\epsilon}^{\dagger}$ is sufficiently small with respect to $\left(\omega_{\epsilon}, h_{0}^{(\epsilon)}\right),\left[A_{0}, B_{\epsilon}^{\dagger}\right]$ is also sufficiently small. Corresponding to the decomposition $f=\sum f_{u, u^{\prime}}$, we have $B=\sum B_{u, u^{\prime}}$. Then the following holds:

$$
\left(B_{\epsilon}^{\dagger}\right)_{u, u^{\prime}}=\bar{H}_{u^{\prime}, \epsilon}^{-1 t} \bar{B}_{u^{\prime}, u} \bar{H}_{u, \epsilon}
$$

Here $H_{u, \epsilon}:=H\left(h_{u}^{(\epsilon)}, \mathbf{v}_{u}\right)$. Hence, we obtain the following:

$$
\left(\mathcal{D} B_{\epsilon}^{\dagger}\right)_{u, u^{\prime}} \frac{d \bar{z}}{\bar{z}}=\bar{H}_{u^{\prime}, \epsilon}^{-1} \cdot\left(\mathcal{D}^{t} \bar{B}_{u^{\prime}, u}\right) \cdot \bar{H}_{u, \epsilon}-\bar{H}_{u^{\prime}, \epsilon}^{-1} \mathcal{D} \bar{H}_{u^{\prime}, \epsilon} \cdot\left(B_{\epsilon}^{\dagger}\right)_{u, u^{\prime}}+\left(B_{\epsilon}^{\dagger}\right)_{u, u^{\prime}} \cdot \bar{H}_{u, \epsilon}^{-1} \mathcal{D} \bar{H}_{u, \epsilon}
$$

Since $B$ is holomorphic, we have $\bar{H}_{u^{\prime}, \epsilon}^{-1} \cdot\left(\mathcal{D}^{t} \bar{B}_{u^{\prime}, u}\right) \cdot \bar{H}_{u, \epsilon} \cdot d \bar{z} / \bar{z}=0$. We put $H_{u \epsilon}^{\prime}:=$ $H\left(h_{u}^{\prime(\epsilon)}, \mathbf{v}_{u}\right)$. Then we have $H_{u, \epsilon}=|z|^{-2 a} H_{u, \epsilon}^{\prime}$, and the following holds with respect to $h_{0}^{(\epsilon)}$ due to Lemma 4.10:

$$
\bar{H}_{u, \epsilon}^{-1} \mathcal{D} \bar{H}_{u, \epsilon}=-a\left(\lambda \frac{d z}{z}+\frac{d \bar{z}}{\bar{z}}\right)+\bar{H}_{u, \epsilon}^{\prime-1} \mathcal{D} \bar{H}_{u, \epsilon}^{\prime}=O(1) \frac{d z}{z}+O(1) \frac{d \bar{z}}{\bar{z}}
$$

Since $\left(B_{\epsilon}^{\dagger}\right)_{u, u^{\prime}}$ is small with respect to $\left(\omega_{\epsilon}, h_{0}^{(\epsilon)}\right),\left(B_{\epsilon}^{\dagger}\right)_{u, u^{\prime}} \cdot \bar{H}_{u, \epsilon}^{-1} \partial \bar{H}_{u, \epsilon}$ is also small. Therefore, $\mathbb{D}_{0}^{\lambda} f^{\dagger} \cdot d \bar{z} / \bar{z}$ is small with respect to $\left(\omega_{\epsilon}, h_{0}^{(\epsilon)}\right)$. It also follows that $\mathbb{D}_{0, \epsilon}^{\lambda \star} f$. $d z / z$ is small. Thus we are done.

\subsection{Proof of Proposition 4.1}

4.5.1 Construction of a family of initial metrics Let $\eta$ be a small positive number such that $\eta<\operatorname{gap}(E, \mathbf{F}) / 10$. Let $\epsilon_{0}$ be a small positive number such that 10 rank $E \cdot \epsilon_{0}<$ $\eta$. For any $0 \leq \epsilon<\epsilon_{0}$, let us take $\omega_{\epsilon}$ be the Kahler forms of $C-D$ with the following properties:

- Let $\left(U_{P}, z\right)$ be a holomorphic coordinate around $P \in D$ such that $z(P)=0$, and then $\omega_{\epsilon}$ is given by (52).

- $\omega_{\epsilon} \longrightarrow \omega_{0}$ for $\epsilon \longrightarrow 0$ in the $C^{\infty}$-sense locally on $X-D$. 
Lemma 4.12 We can construct a family of metrics $h_{0}^{(\epsilon)}$ of $E_{\mid C-D}$ with the following properties:

- $h_{0}^{(\epsilon)}$ is adapted to the perturbed parabolic structure $\mathbf{F}^{(\epsilon)}$.

- $h_{0}^{(\epsilon)} \longrightarrow h_{0}^{(0)}$ in the $C^{\infty}$-sense locally on $C-D$.

- $G\left(h_{0}^{(\epsilon)}\right)$ is uniformly bounded with respect to $\left(\omega_{\epsilon}, h_{0}^{(\epsilon)}\right)$.

- We put $t_{\epsilon}:=\operatorname{det}\left(h_{0}^{(\epsilon)}\right) / \operatorname{det}\left(h_{0}^{(0)}\right)$. Then $t_{\epsilon}$ and $t_{\epsilon}^{-1}$ are bounded independently from $\epsilon$.

Proof We construct a $C^{\infty}$-metric of $E$ on $\bigcup_{P \in D}\left(U_{P}-\{P\}\right)$, by applying the construction given in Section 4.4.2 to $\left(E, \mathbf{F}, \mathbb{D}^{\lambda}\right)_{\mid U_{P}}$ for each $P \in D$, and then we prolong it to a $C^{\infty}$-metric of $E$ on $C-D$.

Let $R\left(\operatorname{det} h_{0}^{(0)}\right)$ be the curvature of the metrized holomorphic bundle $\operatorname{det}\left(E, d^{\prime \prime}, h_{0}^{(0)}\right)$, where $d^{\prime \prime}$ denote the $(0,1)$-part of $\mathbb{D}^{\lambda}$. Since $\operatorname{det} h_{0}^{(0)}$ gives the harmonic metric around $D$ due to our construction, $R\left(\operatorname{det} h_{0}^{(0)}\right)$ vanishes around $D$. We also have $\int R\left(\operatorname{det} h_{0}^{(0)}\right)=-2 \pi \sqrt{-1} \cdot \operatorname{par}-\operatorname{deg}(E, \mathbf{F})=0$. Let us take the $C^{\infty}$-function $\chi_{0}$ on $C$ satisfying $\operatorname{rank}(E) \bar{\partial} \partial \chi_{0}+R\left(\operatorname{det}\left(h_{0}^{(0)}\right)\right)=0$. We put $h_{i n}^{(0)}:=h_{0}^{(0)} \cdot \exp \left(\chi_{0}\right)$. Then $R\left(\operatorname{det} h_{i n}^{(0)}\right)=0$, ie, $\operatorname{det} h_{i n}^{(0)}$ is a harmonic metric of $\operatorname{det}\left(E, \mathbb{D}^{\lambda}\right)$. Let $\chi_{\epsilon}$ be the functions determined by $\operatorname{det}\left(h_{i n}^{(0)}\right)=\operatorname{det}\left(h_{0}^{(\epsilon)}\right) \cdot \exp \left(\operatorname{rank}(E) \cdot \chi_{\epsilon}\right)$. The following claims immediately follows from Lemma 4.12.

- $\left|\chi_{\epsilon}\right|$ are bounded on $C$, independently from $\epsilon$.

- $\chi_{\epsilon} \longrightarrow 0$ in the $C^{\infty}$-sense locally on $C-D$.

We put $h_{i n}^{(\epsilon)}:=h_{0}^{(\epsilon)} \cdot \exp \left(\chi_{\epsilon}\right)$, which is the metric of $E_{\mid C-D}$.

Lemma 4.13 The following claims are easy to check.

- $h_{i n}^{(\epsilon)}$ is adapted to the parabolic structure $\mathbf{F}^{(\epsilon)}$.

- $h_{\text {in }}^{(\epsilon)} \longrightarrow h_{i n}^{(0)}$ in the $C^{\infty}$-sense locally on $C-D$.

- $G\left(h_{i n}^{(\epsilon)}\right)$ is uniformly bounded with respect to $\left(\omega_{\epsilon}, h_{i n}^{(\epsilon)}\right)$.

- $\operatorname{det} h_{i n}^{(\epsilon)}$ is harmonic, and we have $\operatorname{det} h_{i n}^{(\epsilon)}=\operatorname{det} h_{i n}^{(0)}$.

In other words, they give initial metrics for $\left(E, \mathbf{F}^{(\epsilon)}, \mathbb{D}^{\lambda}\right)$ in the sense of Lemma 3.18, and their pseudo-curvatures satisfy uniform finiteness. 
4.5.2 $L_{1}^{2}$-finiteness of the sequence Due to Proposition 2.49, we obtain the harmonic metrics $h^{(\epsilon)}$ for $\left(E, \mathbf{F}^{(\epsilon)}, \mathbb{D}^{\lambda}\right)$ such that $\operatorname{det} h^{(\epsilon)}=\operatorname{det} h_{i n}^{(0)}$. Due to Lemma 2.50, we have the following inequalities for any $\epsilon$ :

$$
M_{\omega_{\epsilon}}\left(h_{i n}^{(\epsilon)}, h^{(\epsilon)}\right) \leq 0 .
$$

Let $s^{(\epsilon)}$ be determined by $h^{(\epsilon)}=h_{i n}^{(\epsilon)} s^{(\epsilon)}$. Due to Lemma 2.45, (53) and $\operatorname{det} s^{(\epsilon)}=1$, there exists a positive constant $A$ which is independent on $\epsilon$, with the following property:

$$
\left|S^{(\epsilon)}\right|_{h_{i n}^{(\epsilon)}} \leq A, \quad\left|\left(s^{(\epsilon)}\right)^{-1}\right|_{h_{i n}^{(\epsilon)}} \leq A
$$

Let $\mathbb{D}_{i n}^{\lambda \star}$ be the operator obtained from $\mathbb{D}^{\lambda}, \omega_{\epsilon}$ and $h_{i n}^{(\epsilon)}$ as in Section 2.2.1. We have the following equalities:

$$
\Delta_{\omega_{\epsilon}}^{\lambda} \operatorname{tr} s^{(\epsilon)}=-\sqrt{-1} \operatorname{tr}\left(s^{(\epsilon)} \Lambda_{\omega_{\epsilon}} G\left(h_{i n}^{(\epsilon)}\right)\right)+\sqrt{-1} \operatorname{tr}\left(\Lambda_{\omega_{\epsilon}} \mathbb{D}^{\lambda} s^{(\epsilon)} \cdot\left(s^{(\epsilon)}\right)^{-1} \cdot \mathbb{D}_{i n}^{\lambda \star} s^{(\epsilon)}\right)
$$

See Remark 2.27 for $\Delta_{\omega_{\epsilon}}^{\lambda}$.

Lemma 4.14 We have $\int \Delta_{\omega_{\epsilon}}^{\lambda} \operatorname{tr} s^{(\epsilon)} \mathrm{dvol}_{\omega_{\epsilon}}=0$.

Proof Note that $\mathbb{D}^{\lambda} S^{(\epsilon)}$ is $L^{2}$ with respect to $h^{(\epsilon)}$ and $\omega_{\epsilon}$ according to Proposition 2.49. Then it is easy to obtain the vanishing $\int \Delta_{\omega_{\epsilon}}^{\lambda} \operatorname{tr} s^{(\epsilon)} \mathrm{dvol}_{\omega_{\epsilon}}=0$ by Stokes' formula and Lemma 5.2 of [17].

Then there exists a positive constant $A^{\prime}$ such that the following holds:

$$
\int\left|\mathbb{D}^{\lambda} s^{(\epsilon)} \cdot\left(s^{(\epsilon)}\right)^{-1 / 2}\right|_{h_{i n}^{(\epsilon)}, \omega_{\epsilon}}^{2} \operatorname{dvol}_{\omega_{\epsilon}} \leq A^{\prime}
$$

In particular, we obtain $\left\|\mathbb{D}^{\lambda} S^{(\epsilon)}\right\|_{L^{2}, \omega_{\epsilon}, h_{i n}^{(\epsilon)}}$ is bounded for $0<\epsilon<\epsilon_{0}$.

Remark 4.15 A rather detailed review surrounding the Donaldson functional in Section 2.4 and Section 2.5 is preparation to obtain the boundedness (54).

4.5.3 End of the proof of Proposition 4.1 Let $Q$ be a point of $C-D$. Let $(U, z)$ be a holomorphic coordinate around $Q$ such that $z(Q)=0$ and $U \simeq \Delta=\{z|| z \mid<1\}$. We use the standard metric $g=d z \cdot d \bar{z}$ of $U$. The harmonic bundles $\left(E, \mathbb{D}^{\lambda}, h^{(\epsilon)}\right)$ induce the Higgs bundles $\left(E, \bar{\partial}_{\epsilon}, \theta_{\epsilon}\right)$. We have $\theta_{\epsilon}=f_{\epsilon} \cdot d z$ on $U$. On the other hand, we also obtain $\bar{\partial}_{i n, \epsilon}$ and $\theta_{i n, \epsilon}$ from $\left(E, \mathbb{D}^{\lambda}, h_{i n}^{(\epsilon)}\right)$, although $\bar{\partial}_{i n, \epsilon}\left(\theta_{i n, \epsilon}\right)=0$ may not 
be satisfied. Let $\delta_{i n, \epsilon}^{\prime}$ be the $(1,0)$-operator obtained from $h_{i n}^{(\epsilon)}$ and $d^{\prime \prime}$, as in Section 2.2.1. Then we have the following relation:

$$
\theta_{\epsilon}=\theta_{i n, \epsilon}-\frac{\lambda}{1+|\lambda|^{2}}\left(\left(s^{(\epsilon)}\right)^{-1} \cdot \delta_{i n, \epsilon}^{\prime} s^{(\epsilon)}\right)
$$

Due to (54), (55) and (56), there exists a constant $C_{0}>0$ such that $\int_{U}\left|f_{\epsilon}\right|_{h^{(\epsilon)}}^{2} \operatorname{dvol}{ }_{g}<$ $C_{0}$ holds for any $0<\epsilon<\epsilon_{0}$. Hence, the following inequality holds for some constants $C_{i}>0(i=1,2,3)$ and for any $0<\epsilon<\epsilon_{0}$ :

$$
\int_{U} \log \left|f_{\epsilon}\right|_{h^{(\epsilon)}}^{2} \mathrm{dvol}_{g} \leq C_{1}+\int_{U} C_{2} \cdot\left|f_{\epsilon}\right|_{h^{(\epsilon)}}^{2} \mathrm{dvol}_{g} \leq C_{3}
$$

Recall the fundamental inequality for the Higgs field of a harmonic bundle [18]:

$$
\Delta_{g} \log \left|f_{\epsilon}\right|_{h^{(\epsilon)}}^{2} \leq-\frac{\left|\left[f_{\epsilon}, f_{\epsilon}^{\dagger}\right]\right|_{h^{(\epsilon)}}^{2}}{\left|f_{\epsilon}\right|_{h^{(\epsilon)}}^{2}} \leq 0 .
$$

Due to (57) and (58), there exists a constant $C_{4}>0$ such that the following holds for any $Q^{\prime} \in U(1 / 2):=\{|z|<1 / 2\}$ :

$$
\left|f_{\epsilon}\left(Q^{\prime}\right)\right|_{h_{i n}^{(\epsilon)}}^{2} \leq C_{4}
$$

By using (56), we obtain that $\delta_{i n, \epsilon}^{\prime} s^{(\epsilon)}$ is uniformly bounded with respect to $\left(\omega_{\epsilon}, h_{i n}^{(\epsilon)}\right)$ on $U(1 / 2)$.

Since $\theta_{\epsilon}^{\dagger}$ is the adjoint of $\theta_{\epsilon}$, we obtain the uniform boundedness of $\theta_{\epsilon}^{\dagger}$ on $U(1 / 2)$. Let $\delta_{i n, \epsilon}^{\prime \prime}$ be the operator obtained from $h_{i n}^{(\epsilon)}$ and $d^{\prime}$ as in Section 2.2.1, where $d^{\prime}$ denotes the $(1,0)$-part of $\mathbb{D}^{\lambda}$. Then we also obtain the uniform boundedness of $\delta_{i n, \epsilon}^{\prime \prime} s^{(\epsilon)}$ on $U(1 / 2)$. Hence, $\mathbb{D}_{i n, \epsilon}^{\lambda \star} S^{(\epsilon)}$ is uniformly bounded on $U(1 / 2)$, where

$$
\mathbb{D}_{i n, \epsilon}^{\lambda \star}=\delta_{i n, \epsilon}^{\prime}-\delta_{i n, \epsilon}^{\prime \prime} .
$$

Because

$$
\begin{aligned}
d^{\prime \prime} & =\bar{\lambda}^{-1}\left(\delta_{i n, \epsilon}^{\prime \prime}+\left(1+|\lambda|^{2}\right) \theta_{i n, \epsilon}^{\dagger}\right) \\
d^{\prime} & =\lambda \delta_{i n, \epsilon}^{\prime}+\left(1+|\lambda|^{2}\right) \theta_{i n, \epsilon},
\end{aligned}
$$

and

we also obtain $\mathbb{D}^{\lambda} S^{(\epsilon)}$ are uniformly bounded on $U(1 / 2)$. Recall the formula

$$
\mathbb{D}^{\lambda} \mathbb{D}_{i n}^{\lambda \star} s^{(\epsilon)}=s^{(\epsilon)} \cdot G\left(h_{i n}^{(\epsilon)}\right)+\mathbb{D}^{\lambda} s^{(\epsilon)} \cdot\left(s^{(\epsilon)}\right)^{-1} \cdot \mathbb{D}_{i n}^{\lambda \star} s^{(\epsilon)} .
$$

Thus $\mathbb{D}^{\lambda} \mathbb{D}_{i n}^{\lambda \star} s^{(\epsilon)}$ is also uniformly bounded on $U(1 / 2)$. Therefore, $\left\{s^{(\epsilon)}\right\}$ is $L_{2}^{p}-$ bounded for any $p>1$ and $U(1 / 2)$. By taking an appropriate subsequence $\left(\epsilon_{i}\right), s^{\left(\epsilon_{i}\right)}$ weakly converges to some $\tilde{s}$ in $L_{2}^{p}$ locally on $C-D$. 
It is easy to see that $h_{i n}^{(0)} \cdot \widetilde{s}$ is a harmonic metric. We have $\operatorname{det} \tilde{s}=1$. We also have the boundedness of $\widetilde{s}$ and $\widetilde{s}^{-1}$ with respect to $h_{i n}^{(0)}$. Thus, we have $h_{i n}^{(0)} \cdot \widetilde{s}=h^{(0)}$, ie, the sequence $\left\{h^{\left(\epsilon_{i}\right)}\right\}$ converges to $h^{(0)}$ weakly in $L_{2}^{p}$ locally on $C-D$.

Although we take a subsequence in the above argument, we can conclude that $h^{(\epsilon)}$ converges to $h^{(0)}$ weakly in $L_{2}^{p}$ locally on $C-D$, due to a general argument. We can also obtain the $C^{\infty}$-convergence by a standard bootstrapping. In the above argument, the convergence of $\left\{\theta^{(\epsilon)}\right\}$ is also proved.

Remark 4.16 As for the proof of Proposition 4.2, we take a $C^{\infty}$-metric $h_{\text {in }}$ of $\left(E, \mathbf{F}, \mathbb{D}^{\lambda}\right)$ such that each restriction $h_{i n \mid C_{t}}$ is an initial metric. Let $s$ be determined by $h_{H}=h_{i n} \cdot s$. By applying the same argument, we obtain the continuity of $s$. Similarly for $\theta_{H}$.

\section{Existence of a pluri-harmonic metric}

We will prove our main existence theorem (Theorem 5.16) of a pluri-harmonic metric for a $\mu_{L}$-stable parabolic $\lambda$-flat bundle with trivial characteristic numbers, which is adapted to the parabolic structure. (See Section 3.3 of [14] for adaptedness.) As we recalled in Proposition 2.54, if the dimension of the base space is 1, it was established by Simpson [18].

For the 2-dimensional case, as mentioned in the introduction, the problem is reduced to the convergence of a sequence of Hermitian-Einstein metrics for $\epsilon$-perturbations. To show it, we use a variant of the classical argument by Donaldson in [4; 5]. Namely, we study the convergence of their restrictions to generic curves in Sections 5.1-5.2 by using the result in Section 4 for which the Donaldson functionals played the essential roles. (See also Section 1.2.3 for an outline of the argument.)

The $n$-dimensional case $(n \geq 3)$ can be reduced to the 2-dimensional case (Section 5.3.1). It may remind readers that Mehta and Ramanathan [12] established the Kobayashi-Hitchin correspondence for stable bundles with trivial Chern classes in the $n$-dimensional case ( $n \geq 3$ ) by reducing it to the 2 -dimensional case.

As a consequence of Theorem 5.16, we explain an equivalence of $\mu_{L}$-polystable regular filtered $\lambda$-flat bundles for various $\lambda$ in Section 5.3.2, and the existence of Corlette-Jost-Zuo metric in Section 5.3.3.

\subsection{Preliminary}

Let $C$ be a smooth projective curve with a finite subset $D$. Let $\left(E, \mathbf{F}, \mathbb{D}^{\lambda}\right)$ be a stable parabolic $\lambda$-flat bundle on $(C, D)$ with $\operatorname{par}-\operatorname{deg}(E, \mathbf{F})=0$. For each $P \in D$, let 
$\left(U_{\boldsymbol{P}}, z\right)$ be a holomorphic coordinate around $P$ such that $z(P)=0$. Let $\mathbf{F}^{(\epsilon)}$ be an $\epsilon$-perturbation as in (II) of Section 2.1.6 for $\epsilon=m^{-1}$. We have harmonic metrics $h_{0}^{(\epsilon)}$ for $\left(E, \mathbf{F}^{(\epsilon)}, \mathbb{D}^{\lambda}\right)$. We assume $\operatorname{det} h_{0}^{(\epsilon)}=\operatorname{det} h_{0}^{(0)}$. As shown in Proposition 4.1, the sequence $\left\{h_{0}^{(\epsilon)}\right\}$ converges to $h_{0}^{(0)}$ in the $C^{\infty}$-sense locally on $C-D$. Let $N$ be a large positive number, for example $N>10$. In this subsection, we use Kahler metrics $g_{\epsilon}(\epsilon \geq 0)$ of $C-D$ which are as follows on $U_{P}$ for each $P \in D$ :

$$
\left(\epsilon^{N+2}|z|^{2 \epsilon}+|z|^{2}\right) \frac{d z \cdot d \bar{z}}{|z|^{2}}
$$

We assume that the sequence $\left\{g_{\epsilon}\right\}$ converges to $g_{0}$ for $\epsilon \longrightarrow 0$ in the $C^{\infty}$-sense locally on $C-D$.

Proposition 5.1 Let $h^{(\epsilon)}(\epsilon>0)$ be hermitian metrics of $E_{\mid C-D}$ with the following properties:

(1) Let $s^{(\epsilon)}$ be determined by $h^{(\epsilon)}=h_{0}^{(\epsilon)} \cdot s^{(\epsilon)}$. Then $s^{(\epsilon)}$ is bounded with respect to $h_{0}^{(\epsilon)}$, and we have $\operatorname{det} s^{(\epsilon)}=1$. It also satisfies the finiteness $\left\|\mathbb{D}^{\lambda} s^{(\epsilon)}\right\|_{2, h_{0}^{(\epsilon)}, g_{\epsilon}}<$ $\infty$. (The estimates may depend on $\epsilon$.)

(2) $\left\|G\left(h^{(\epsilon)}\right)\right\|_{2, h^{(\epsilon)}, g_{\epsilon}}<\infty$ and $\lim _{\epsilon \rightarrow 0}\left\|G\left(h^{(\epsilon)}\right)\right\|_{2, h^{(\epsilon)}, g_{\epsilon}}=0$.

Then the following claims hold.

- The sequence $\left\{s^{(\epsilon)}\right\}$ is weakly convergent to the identity in $L_{1}^{2}$ locally on $C-D$.

- $\left|s^{(\epsilon)}\right|_{h_{0}^{(\epsilon)}}$ and $\left|\left(s^{(\epsilon)}\right)^{-1}\right|_{h_{0}^{(\epsilon)}}$ are bounded on $C-D$ uniformly in $\epsilon$.

Proof In some sense, the proposition means that the sequence $\left\{s^{(\epsilon)}\right\}$ cannot bubble off, which heuristically follows from the assumption that the energies go to 0 .

To begin with, we remark that we have only to show the existence of a subsequence $\left\{s^{\left(\epsilon_{i}\right)}\right\}$ with the desired properties as above. We put

$$
\left\|S^{(\epsilon)}\right\|_{\infty, h_{0}^{(\epsilon)}}:=\sup _{P \in C-D}\left|s_{\mid P}^{(\epsilon)}\right|_{h_{0}^{(\epsilon)}}
$$

For any point $P \in C-D$, let $S E\left(s^{(\epsilon)}\right)(P)$ denote the maximal eigenvalue of $s_{\mid P}^{(\epsilon)}$. There exists a constant $0<C_{1}<1$ such that

$$
C_{1} \cdot\left|s_{\mid P}^{(\epsilon)}\right|_{h_{0}^{(\epsilon)}} \leq S E\left(s^{(\epsilon)}\right)(P) \leq\left|s_{\mid P}^{(\epsilon)}\right|_{h_{0}^{(\epsilon)}} .
$$

We have $\operatorname{det} s_{\mid P}^{(\epsilon)}=1$. Hence, it is easy to see $\log \operatorname{tr} s_{\mid P}^{(\epsilon)} \geq \log \operatorname{rank}(E) \geq 0$. We also have $S E\left(s^{(\epsilon)}\right)(P) \geq 1$ for any $P$. 
Let us take $b_{\epsilon}>0$ satisfying $2 \leq b_{\epsilon} \cdot \sup S E\left(s^{(\epsilon)}\right)(P) \leq 2+\epsilon$. We put $\widetilde{s}^{(\epsilon)}=b_{\epsilon} S^{(\epsilon)}$ and $\widetilde{h}^{(\epsilon)}:=h_{0}^{(\epsilon)} \cdot \widetilde{s}^{(\epsilon)}$. Then $\widetilde{s}^{(\epsilon)}$ are uniformly bounded with respect to $h_{0}^{(\epsilon)}$. We remark $G\left(\tilde{h}^{(\epsilon)}\right)=G\left(h^{(\epsilon)}\right)$. We also remark that $h^{(\epsilon)}$ and $\widetilde{h}^{(\epsilon)}$ induce the same metric of $\operatorname{End}(E)$.

Lemma 5.2 After going to an appropriate subsequence, $\left\{\widetilde{s}^{\left(\epsilon_{i}\right)}\right\}$ converges to a positive constant multiplication, weakly in $L_{1}^{2}$ locally on $C-D$.

Proof We have the following (Section 2.2.5):

$$
\Delta_{g_{0}, h_{0}^{(\epsilon)}}^{\lambda} \widetilde{s}^{(\epsilon)}=\widetilde{s}^{(\epsilon)} \sqrt{-1} \Lambda_{g_{0}} G\left(\widetilde{h}^{(\epsilon)}\right)+\sqrt{-1} \Lambda_{g_{0}} \mathbb{D}^{\lambda} \widetilde{s}^{(\epsilon)}\left(\widetilde{s}^{(\epsilon)}\right)^{-1} \mathbb{D}_{h_{0}^{(\epsilon)}}^{\lambda \star} \tilde{s}^{(\epsilon)}
$$

We can show $\int \Delta_{g_{0}}^{\lambda} \operatorname{tr} \widetilde{s}^{(\epsilon)} \cdot \operatorname{dvol}_{g_{0}}=0$ by the same argument as the proof of Lemma 4.14. We obtain the following inequality from (59) and the uniform boundedness of $\tilde{S}^{(\epsilon)}$ :

$$
\begin{aligned}
\int\left|\mathbb{D}^{\lambda} \widetilde{s}^{(\epsilon)} \cdot\left(\widetilde{s}^{(\epsilon)}\right)^{-1 / 2}\right|_{g_{0}, h_{0}^{(\epsilon)}}^{2} & \operatorname{dvol}_{g_{0}} \leq A \cdot \int\left|\operatorname{tr} \Lambda_{g_{0}} G\left(\tilde{h}^{(\epsilon)}\right)\right| \cdot \operatorname{dvol}_{g_{0}} \\
=A \cdot \int\left|\operatorname{tr} \Lambda_{g_{\epsilon}} G\left(\widetilde{h}^{(\epsilon)}\right)\right| \cdot \operatorname{dvol}_{g_{\epsilon}} \leq A^{\prime} \cdot\left\|G\left(\widetilde{h}^{(\epsilon)}\right)\right\|_{2, \widetilde{h}^{(\epsilon)}, g_{\epsilon}} &
\end{aligned}
$$

In particular, we obtain the uniform estimate

$$
\left\|\mathbb{D}^{\lambda} \widetilde{s}^{(\epsilon)}\right\|_{2, g_{0}, h_{0}^{(\epsilon)}}^{2} \leq A^{\prime \prime} \cdot\left\|G\left(\widetilde{h}^{(\epsilon)}\right)\right\|_{2, \widetilde{h}^{(\epsilon)}, g_{\epsilon}} .
$$

Therefore, the sequence $\left\{\widetilde{S}^{(\epsilon)}\right\}$ is $L_{1}^{2}$-bounded on any compact subset of $C-D$. By taking an appropriate subsequence, it is weakly $L_{1}^{2}$-convergent locally on $C-D$. Let $\widetilde{S}^{(\infty)}$ denote the weak limit. We obtain $\mathbb{D}^{\lambda} \widetilde{S}^{(\infty)}=0$. We also know that $\widetilde{s}^{(\infty)}$ is bounded with respect to $h_{0}^{(0)}$. Therefore, $\widetilde{s}^{(\infty)}$ gives an automorphism of $\left(E, \mathbf{F}, \mathbb{D}^{\lambda}\right)$. Due to the stability of $\left(E, \mathbf{F}, \mathbb{D}^{\lambda}\right), \widetilde{s}^{(\infty)}$ is a constant multiplication.

We would like to show $\widetilde{s}^{(\infty)} \neq 0$. Let us take any point $Q_{\epsilon} \in C-D$ satisfying the following:

$$
S E\left(s^{(\epsilon)}\right)\left(Q_{\epsilon}\right) \geq \frac{9}{10} \cdot \sup _{P \in C-D} S E\left(s^{(\epsilon)}\right)(P)
$$

Then we have $\log \operatorname{tr} \widetilde{S}^{(\epsilon)}\left(Q_{\epsilon}\right) \geq \log (9 / 5)$. By taking an appropriate subsequence, we may assume the sequence $\left\{Q_{\epsilon}\right\}$ converges to a point $Q_{\infty}$. There are two cases (i) $Q_{\infty} \in D$, (ii) $Q_{\infty} \notin D$. We argue only the case (i). The other case can be argued similarly and more easily.

We use the coordinate neighbourhood $(U, z)$ such that $z\left(Q_{\infty}\right)=0$. For any point $P \in U$, we put $\Delta(P, r):=\{Q \in U|| z(P)-z(Q) \mid<r\}$. When $\epsilon$ is sufficiently small, 
$Q_{\epsilon}$ is contained in $\Delta\left(Q_{\infty}, 1 / 2\right)=\{|z|<1 / 2\}$. Let $g=d z \cdot d \bar{z}$ denote the standard metric of $U$. We have the following inequality on $U-\left\{Q_{\infty}\right\}$ (see Section 2.2.5):

$$
\Delta_{g}^{\lambda} \log \operatorname{tr} \widetilde{s}^{(\epsilon)} \leq\left|\Lambda_{g} G\left(\widetilde{h}^{(\epsilon)}\right)\right|_{\widetilde{h}^{(\epsilon)}}
$$

Let $B^{(\epsilon)}$ be the endomorphism of $E$ determined as follows:

$$
G\left(\tilde{h}^{(\epsilon)}\right)=G\left(h^{(\epsilon)}\right)=B^{(\epsilon)} \frac{d z \cdot d \bar{z}}{|z|^{2}}
$$

Then we have the following estimate for some constant $A>0$ which is independent of $\epsilon$ :

$$
\int\left|B^{(\epsilon)}\right|_{\widetilde{h}_{0}^{(\epsilon)}}^{2}\left(\epsilon^{N+1}|z|^{2 \epsilon}+|z|^{2}\right)^{-1} \frac{\mathrm{dvol}_{g}}{|z|^{2}} \leq A \int\left|G\left(\widetilde{h}^{(\epsilon)}\right)\right|_{\widetilde{h}^{(\epsilon)}, g_{\epsilon}}^{2} \operatorname{dvol}_{g_{\epsilon}}
$$

Here $A$ denotes a constant independent of $\epsilon$. Due to Proposition 2.16 in [14], there exist functions $v^{(\epsilon)}$ such that the following inequalities hold for some constant $A^{\prime}>0$ which is independent of $\epsilon$ :

$\bar{\partial} \partial v^{(\epsilon)}=\left|B^{(\epsilon)}\right|_{\widetilde{h}^{(\epsilon)}} \frac{d z \cdot d \bar{z}}{|z|^{2}}, \quad\left|v^{(\epsilon)}(z)\right| \leq A^{\prime} \cdot\left(\epsilon^{(N-1) / 2}|z|^{\epsilon}+|z|^{1 / 2}\right) \cdot\left\|G\left(\widetilde{h}^{(\epsilon)}\right)\right\|_{2, \widetilde{h}^{(\epsilon)}, g_{\epsilon}}$

Then we have $\Delta_{g}^{\lambda}\left(\log \operatorname{tr} \widetilde{s}^{(\epsilon)}-v^{(\epsilon)}\right) \leq 0$ on $U-\left\{Q_{\infty}\right\}$. Since $\log \operatorname{tr} \widetilde{s}^{(\epsilon)}-v^{(\epsilon)}$ is bounded from above, the inequality holds on $U$. Therefore, we obtain the following:

$$
\log \operatorname{tr} \tilde{s}^{(\epsilon)}\left(Q_{\epsilon}\right)-v^{(\epsilon)}\left(Q_{\epsilon}\right) \leq A^{\prime \prime} \cdot \int_{\Delta\left(Q_{\epsilon}, 1 / 2\right)}\left(\log \operatorname{tr} \tilde{s}^{(\epsilon)}-v^{(\epsilon)}\right) \cdot \operatorname{dvol} g
$$

Here $A^{\prime \prime}>0$ denotes a constant. Due to the uniform boundedness of $v^{(\epsilon)}$, we obtain the following inequalities, for some positive constants $C_{i}(i=1,2)$ which are independent of $\epsilon$ :

$$
\log (9 / 5) \leq \log \operatorname{tr} \widetilde{s}^{(\epsilon)}\left(Q_{\epsilon}\right) \leq C_{1} \cdot \int_{\Delta\left(Q_{\epsilon}, 1 / 2\right)} \log \operatorname{tr} \widetilde{s}^{(\epsilon)} \cdot \operatorname{dvol}_{g}+C_{2}
$$

Recall that $\log \operatorname{tr} \widetilde{s}^{(\epsilon)}$ are uniformly bounded from above. Therefore, there exists a positive constant $C_{3}$ such that the following holds for any sufficiently small $\epsilon>0$ :

$$
\int_{\Delta\left(Q_{\epsilon}, 1 / 2\right)}-\min \left(0, \log \operatorname{tr} \widetilde{s}^{(\epsilon)}\right) \cdot \operatorname{dvol}_{g} \leq C_{3}
$$

Due to Fatou's lemma, we obtain the following:

$$
\int_{\Delta\left(Q_{\infty}, 1 / 2\right)}-\min \left(0, \log \operatorname{tr} \widetilde{s}^{(\infty)}\right) \cdot \operatorname{dvol}_{g} \leq C_{3}
$$


It means $\widetilde{S}^{(\infty)}$ is not constantly 0 on $\Delta\left(Q_{\infty}, 1 / 2\right)$. In all, we can conclude that $\widetilde{s}^{(\infty)}$ is a positive constant multiplication. Thus, the proof of Lemma 5.2 is accomplished.

Let $\left\{\widetilde{s}^{\left(\epsilon_{i}\right)}\right\}$ be a subsequence as in Lemma 5.2. It is almost everywhere convergent to a constant multiplication. Then we obtain that the sequence $\left\{\operatorname{det} \widetilde{s}^{\left(\epsilon_{i}\right)}=b_{\epsilon_{i}}^{\operatorname{rank} E} \cdot \operatorname{id}_{\operatorname{det}(E)}\right\}$ converges to a positive constant. In particular, $\left\{b_{\epsilon_{i}}\right\}$ is convergent. Therefore, the sequence $\left\{s^{\left(\epsilon_{i}\right)}\right\}$ is convergent to the identity. Moreover, $S E\left(s^{(\epsilon)}\right)$ are bounded on $C-D$ uniformly in $\epsilon$. Hence, $\mid s^{\left(\epsilon_{i}\right)} h_{0}^{(\epsilon)}$ are bounded on $C-D$ uniformly in $\epsilon$. Because $\operatorname{det}\left(s^{\left(\epsilon_{i}\right)}\right)=1$, we also obtain the uniform boundedness of $\left|\left(s^{\left(\epsilon_{i}\right)}\right)^{-1}\right|_{h_{0}^{(\epsilon)}}$. Thus the proof of Proposition 5.1 is finished.

\section{Corollary 5.3}

- The sequence $\left\{h^{(\epsilon)}\right\}$ is convergent to $h_{0}^{(0)}$ weakly in $L_{1}^{2}$ locally on $C-D$.

- The sequence $\left\{\mathbb{D}^{\lambda} S^{(\epsilon)}\right\}$ is weakly convergent to 0 in $L^{2}$ locally on $C-D$.

- The sequence $\left\{\theta^{(\epsilon)}\right\}$ converges to $\theta^{(0)}$ is weakly convergent to 0 in $L^{2}$ locally on $C-D$.

- In particular, the sequences are convergent almost everywhere.

\subsection{The surface case}

5.2.1 Statement Let $X$ be a smooth irreducible projective surface with an ample line bundle $L$, and let $D$ be a simple normal crossing hypersurface with the irreducible decomposition $D=\bigcup_{i \in S} D_{i}$. We put $X^{*}:=X-D$. Let $\mathbf{c}$ be any element of $\mathbf{R}^{S}$. Let $\left(E, \mathbf{F}, \mathbb{D}^{\lambda}\right)$ be a $\mu_{L}$-stable $\mathbf{c}$-parabolic $\lambda$-flat bundle on $(X, D)$ with trivial characteristic numbers par-deg ${ }_{L}(E, \mathbf{F})=\int_{X} \operatorname{par} \operatorname{ch}_{2}(E, \mathbf{F})=0$. Recall that it implies par-c $c_{1}(E, \mathbf{F})=0$, due to Bogomolov-Gieseker inequality and Hodge index theorem. (See Corollary 6.2 of [14].) Recall that the problem can be reduced to the classical Hodge-de Rham theory in the rank one case.

Lemma 5.4 There exists a pluri-harmonic metric $h_{\operatorname{det}(E)}$ for the determinant bundle $\operatorname{det}\left(E, \mathbf{F}, \mathbb{D}^{\lambda}\right)$. It is unique up to constant multiplications.

Proof We take an ordinary metric $h_{1}$ for $\operatorname{det}\left(E, \mathbf{F}, \mathbb{D}^{\lambda}\right)$. By construction, we may assume that $R\left(h_{1}\right)$ is $C^{\infty}(1,1)$-form on $X$. By Lemma 3.23 and par-c ${ }_{1}(E, \mathbf{F})=0$, the cohomology class of $R\left(h_{1}\right)$ is 0 . Hence, we can take a $C^{\infty}$-function $\chi$ on $X$ such that $\bar{\partial} \partial \chi=R\left(h_{1}\right)$. We set $h_{\operatorname{det}(E)}:=h_{1} \cdot e^{-\chi}$. Then we have $G\left(h_{\operatorname{det}(E)}\right)=$ $\left(1+|\lambda|^{2}\right) \cdot R\left(h_{1} \cdot e^{-\chi}\right)=0$ due to Lemma 2.31. The uniqueness is also easy, or already contained in Proposition 2.55 . 
The purpose of this subsection is to show the following existence theorem.

Theorem 5.5 There exists a tame pluri-harmonic metric $h$ of $\left(E, \mathbb{D}^{\lambda}\right)_{\mid X^{*}}$ with $\operatorname{det}(h)=h_{\operatorname{det} E}$ which is adapted to the parabolic structure.

The proof will be given in the rest of this subsection.

\subsubsection{A sequence of Hermitian-Einstein metrics for the $\epsilon$-perturbations Let} $\mathbf{F}^{(\epsilon)}$ be an $\epsilon$-perturbation as in (II) of Section 2.1.6. If $\epsilon$ is sufficiently small, $\left(E, \mathbf{F}^{(\epsilon)}, \mathbb{D}^{\lambda}\right)$ is also $\mu_{L}$-stable. We also have par-c ${ }_{1}\left(E, \mathbf{F}^{(\epsilon)}\right)=\operatorname{par} \mathrm{c}_{1}(E, \mathbf{F})=0$. Since $\left(E, \mathbf{F}^{(\epsilon)}, \mathbb{D}^{\lambda}\right)$ is graded semisimple and satisfies the SPW-condition by the construction in (II) of Section 2.1.6, we can apply Proposition 3.19, and we can take the hermitian metric $h^{(\epsilon)}$ of $E_{\mid X-D}$ such that (i) adapted to $\mathbf{F}^{(\epsilon)}$, (ii) $\operatorname{det} h^{(\epsilon)}=h_{\operatorname{det}(E)}$, (iii) $\Lambda_{\omega_{\epsilon}} G\left(h^{(\epsilon)}\right)=0$, where $\omega_{\epsilon}$ are given as in (29).

Since $h_{\operatorname{det}(E)}$ is pluri-harmonic, we also have $\operatorname{tr} G\left(h^{(\epsilon)}\right)=0$. Therefore, we have the following convergence:

$$
\begin{aligned}
\left(\frac{\sqrt{-1}}{2 \pi}\right)^{2} \int\left|G\left(h^{(\epsilon)}\right)\right|_{h^{(\epsilon)}, \omega_{\epsilon}}^{2} \operatorname{dvol}_{\omega_{\epsilon}} & =\left(\frac{\sqrt{-1}}{2 \pi}\right)^{2} \int \operatorname{tr}\left(G\left(h^{(\epsilon)}\right)^{2}\right) \\
& =2\left(1+|\lambda|^{2}\right)^{2} \cdot \operatorname{par}_{-c_{2}}\left(E, \mathbf{F}^{(\epsilon)}\right) \longrightarrow 0
\end{aligned}
$$

Let us study the convergence of $h^{(\epsilon)}$ as $\epsilon \rightarrow 0$.

5.2.3 Convergence on almost every curve Let $L^{m}$ be sufficiently ample. We put $\mathbb{P}_{m}:=\mathbb{P}\left(H^{0}\left(X, L^{m}\right)^{\vee}\right)$. For any $s \in \mathbb{P}_{m}$, we put $X_{s}:=s^{-1}(0)$. Recall Proposition 2.21, and let $\mathcal{U}$ denote the nonempty Zariski open subset of $\mathbb{P}_{m}$ which consists of the points $s$ with the following properties:

- $X_{S}$ is smooth, and $X_{S} \cup D$ is a simple normal crossing hypersurface.

- $\left(E, \mathbf{F}, \mathbb{D}^{\lambda}\right)_{\mid X_{S}}$ is $\mu_{L}$-stable.

In the following, $\epsilon$ are assumed to be sufficiently small, such that $\left(E, \mathbf{F}^{(\epsilon)}, \mathbb{D}^{\lambda}\right)_{\mid X_{s}}$ are $\mu_{L}$-stable for any $s \in \mathcal{U}$.

We set $X_{s}^{*}:=X_{S} \backslash D$ and $D_{s}:=X_{S} \cap D$. We have the metric $\omega_{\epsilon, s}$ of $X_{S}^{*}$, induced by $\omega_{\epsilon}$. The induced volume form is denoted by $\mathrm{dvol}_{s}$. We put $\left(E_{s}, \mathbf{F}_{s}, \mathbb{D}_{s}^{\lambda}\right):=\left(E, \mathbf{F}, \mathbb{D}^{\lambda}\right)_{\mid X_{s}}$.

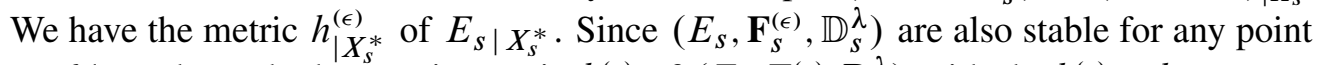
$s \in \mathcal{U}$, we have the harmonic metric $h_{s}^{(\epsilon)}$ of $\left(E_{s}, \mathbf{F}_{s}^{(\epsilon)}, \mathbb{D}_{s}^{\lambda}\right)$ with $\operatorname{det} h_{s}^{(\epsilon)}=h_{\operatorname{det} E} \mid X_{s}^{*}$. Let $u_{s}^{(\epsilon)}$ be the endomorphism of $E_{\mid X_{s}^{*}}$ determined by $h_{\mid X_{s}^{*}}^{(\epsilon)}=h_{s}^{(\epsilon)} \cdot u_{s}^{(\epsilon)}$. Let $\theta_{s}^{(\epsilon)}$ denote the associated Higgs field. For a point $x \in X^{*}$, we put $\mathcal{U}_{x}:=\left\{s \in \mathcal{U} \mid x \in X_{s}\right\}$. 
We put $Z:=\left\{x \in X^{*} \mid \mathcal{U}_{x}=\varnothing\right\}$. We remark that $Z$ is a finite set. Let us fix a sequence $\epsilon_{i} \longrightarrow 0$. We often use the symbol " $\epsilon$ " instead of " $\epsilon_{i}$ ", to simplify the description. We set $\mathbb{D}_{s}^{\lambda}:=\mathbb{D}_{\mid X_{s}^{*}}^{\lambda}$

Lemma 5.6 For almost every $s \in \mathcal{U}$, the following holds:

- We have the following convergence as $\epsilon \longrightarrow 0$ :

$$
\int_{X_{s}}\left|G\left(h_{\mid X_{S}}^{(\epsilon)}\right)\right|_{h_{s}^{(\epsilon)}, \omega_{\epsilon}}^{2} \mathrm{dvol}_{s} \longrightarrow 0
$$

- For each $\epsilon$, we have the finiteness:

$$
\left\|\mathbb{D}_{s}^{\lambda} u_{s}^{(\epsilon)}\right\|_{L^{2}, h_{s}^{(\epsilon)}, \omega_{\epsilon}}<\infty
$$

Let $\widetilde{\mathcal{U}}$ denote the set of $s$ for which both (61) and (62) hold.

Proof It can be shown by the argument in the proof of Lemma 9.3 of [14]. ( $\mathcal{Z}_{2}$ should be corrected to $\left\{(x, s, t) \in X \times U_{1} \times \mathcal{B} \mid\left(t s_{2}+(1-t) s\right)(x)=0\right\}$.) We give only a brief explanation. The first claim is heuristically clear from (60). Let us explain the second claim. Let $h_{i n}^{(\epsilon)}$ be an initial metric for $\left(E, \mathbf{F}^{(\epsilon)}, \mathbb{D}^{\lambda}\right)$ as in Lemma 3.18. Let $w^{(\epsilon)}$ be determined by $h^{(\epsilon)}=h_{i n}^{(\epsilon)} \cdot w^{(\epsilon)}$. According to Proposition 3.19, $h_{i n}^{(\epsilon)}$ and $h^{(\epsilon)}$ are mutually bounded, and $\mathbb{D}^{\lambda} w^{(\epsilon)}$ is $L^{2}$ with respect to $h^{(\epsilon)}$ and $\omega_{\epsilon}$. Hence, we obtain (i) $h_{\mid X_{s}^{*}}^{(\epsilon)}$ and $h_{i n \mid X_{s}^{*}}^{(\epsilon)}$ are mutually bounded, (ii) $\mathbb{D}_{s}^{\lambda} w_{\mid X_{s}^{*}}^{(\epsilon)}$ is $L^{2}$ with respect to $h_{\mid X_{s}^{*}}^{(\epsilon)}$ and $\omega_{\epsilon}$ for almost all $s$. Let $v_{s}^{(\epsilon)}$ be determined by $h_{s}^{(\epsilon)}=X_{i n \mid X_{s}^{*}}^{(\epsilon)} \cdot v_{s}^{(\epsilon)}$. Applying Proposition 3.19 to $\left(E_{s}, \mathbf{F}_{s}, \mathbb{D}_{s}^{\lambda}\right)$, and using the uniqueness of a harmonic metric, we obtain (i) $h_{s}^{(\epsilon)}$ and $h_{i n \mid X_{s}^{*}}^{(\epsilon)}$ are mutually bounded, (ii) $\mathbb{D}_{s}^{\lambda} v_{s}^{(\epsilon)}$ is $L^{2}$ with respect to $h_{s}^{(\epsilon)}$ and $\omega_{s}$. Because $u_{s}^{(\epsilon)}=\left(w_{\mid X_{s}^{*}}^{(\epsilon)}\right)^{-1} \cdot v_{s}$, the second claim follows.

Recall $h_{s}^{(0)}$ is a harmonic metric for $\left(E_{s}, \mathbf{F}_{s}, \mathbb{D}_{s}^{\lambda}\right)$ such that $\operatorname{det}\left(h_{s}^{(0)}\right)=h_{\operatorname{det}(E) \mid X_{s}^{*} \text {, and }}$ $\theta_{s}^{(0)}$ denotes the associated Higgs field. We obtain the following claims from Corollary 5.3 and Lemma 5.6.

Corollary 5.7 For any $s \in \widetilde{\mathcal{U}}$, the sequence $\left\{h_{\mid X_{s}^{*}}^{(\epsilon)}\right.$ converges to $h_{s}^{(0)}$ weakly in $L_{1}^{2}$ locally on $X_{s}^{*}$, and $\left\{\theta_{\mid X_{s}^{*}}^{(\epsilon)}\right\}$ converges to $\theta_{s}^{(0)}$ weakly in $L^{2}$ locally on $X_{s}^{*}$. In particular, they are almost everywhere convergent. 
5.2.4 Construction of a metric defined almost everywhere Let us take any Kahler form $\omega_{\mathbb{P}_{m}}$ of $\mathbb{P}_{m}$. We put $\mathcal{Z}:=\left\{(s, x) \in \mathcal{U} \times X^{*} \mid x \in X_{s}\right\}$. Then we have the induced metric of $\mathcal{Z}$. The induced volume form is denoted by $\operatorname{dvol}_{\mathcal{Z}}$. Let $\mathcal{T}$ denote the set of $(s, x) \in \widetilde{\mathcal{U}} \times X$ such that (i) $(s, x) \in \mathcal{Z}$, (ii) $\lim _{\epsilon \rightarrow 0} h_{\mid x}^{(\epsilon)}=h_{s \mid x}^{(0)}$, where $h_{s}^{(0)}$ is as in Corollary 5.7 .

Lemma 5.8 The measure of $\mathcal{Z}-\mathcal{T}$ is 0 with respect to $\operatorname{dvol}_{\mathcal{Z}}$.

Proof Let us consider the naturally defined fibration $\mathcal{Z} \longrightarrow \mathcal{U}$. Then the claim follows from Corollary 5.7 and Fubini's theorem.

Lemma 5.9 For almost every $x \in X^{*}$ and almost every $s \in \mathcal{U}_{x}$, the sequence $\left\{h_{\mid x}^{(\epsilon)}\right\}$ converges to $h_{S \mid x}^{(0)}$.

Proof Let us consider the naturally defined fibration $\mathcal{T} \longrightarrow X^{*}$. Then the claim follows from Lemma 5.8 and Fubini's theorem.

Let $\mathcal{V}$ denote the set of $x \in X^{*}$ such that the sequence $\left\{h_{\mid x}^{(\epsilon)}\right\}$ converges to $h_{s \mid x}^{(0)}$ for almost $s \in \mathcal{U}_{x}$. For any $x \in \mathcal{V}$, let $\widetilde{\mathcal{U}}_{x}$ denote the set of $s$ such that $\left\{h_{\mid x}^{(\epsilon)}\right\}$ converges to $h_{s \mid x}^{(0)}$.

Lemma 5.10 For any $x \in \mathcal{V}$ and for any $s_{i} \in \widetilde{\mathcal{U}}_{x}(i=1,2)$, we have $h_{s_{1} \mid x}^{(0)}=h_{s_{2} \mid x}^{(0)}$.

Proof Both of them are the same as the $\operatorname{limit}_{\lim _{\epsilon \rightarrow 0}} h_{x}^{(\epsilon)}$.

Let us take any $x \in \mathcal{V}$ and any $s \in \widetilde{\mathcal{U}}_{x}$. Then the metric $h_{x}$ of $E_{\mid x}$ is given by $h_{x}:=h_{s \mid x}^{(0)}$. Due to Lemma 5.10, it is well defined. Thus, we obtain the metric $h_{\mathcal{V}}:=\left(h_{x} \mid x \in \mathcal{V}\right)$ of $E_{\mid \mathcal{V}}$.

5.2.5 The $C^{1}$-property We would like to show that $h_{\mathcal{V}}$ is $C^{1}$ on $X^{*}-Z$, in other words, we would like to show the existence of a $C^{1}$-metric $h$ of $E_{\mid X^{*}-Z}$ such that $h=h_{\mathcal{V}}$ on $\mathcal{V}$. Let us begin with a preparation.

Lemma 5.11 Let $x \in X^{*}-Z$. Let us take any $s \in \mathcal{U}_{x}$. Then there exists a Lefschetz fibration $\varphi: \tilde{X} \longrightarrow \mathbb{P}^{1}$ with the following properties:

- $x$ is not a singular point of $\varphi$.

- $\varphi^{-1}(0)=X_{s}$.

- Almost every $t \in \mathbb{P}^{1}$ belongs to $\widetilde{\mathcal{U}}$. 
Proof Let $\mathcal{M}$ denote the set of the lines $\ell$ of $\mathbb{P}_{m}$ which contain $s$. We put

$$
\widehat{\mathbb{P}}_{m}:=\left\{\left(\ell, s^{\prime}\right) \in \mathcal{M} \times \mathbb{P}_{m} \mid s^{\prime} \in \ell\right\} \subset \mathcal{M} \times \mathbb{P}_{m} .
$$

It is the blow up of $\mathbb{P}_{m}$ at $s$. We have the projection $\pi_{2}: \widehat{\mathbb{P}}_{m} \longrightarrow \mathbb{P}_{m}$. We put $\mathcal{U}^{*}:=\pi_{2}^{-1}(\mathcal{U})$ and $\widetilde{\mathcal{U}}^{*}:=\pi_{2}^{-1}(\widetilde{\mathcal{U}})$. Since $\mathcal{U}-\widetilde{\mathcal{U}}$ has measure 0 , the measure of $\widehat{\mathbb{P}}_{m}-\widetilde{\mathcal{U}}^{*}$ is also 0 . Let us consider the projection $\pi_{1}: \widehat{\mathbb{P}}_{m} \longrightarrow \mathcal{M}$, and apply Fubini's theorem. Then for almost every $\ell \in \mathcal{M}$ and for almost every $s_{1} \in \ell$, we have $s_{1} \in \widetilde{\mathcal{U}}^{*}$. Thus we are done.

Let $x$ be any point of $X^{*}-Z$. Let us take a Lefschetz fibration $\pi_{i}: \tilde{X}_{i} \longrightarrow \mathbb{P}^{1}$ $(i=1,2)$ with the following properties:

- Both of them satisfy the properties in Lemma 5.11.

- Around $x$, the fibers of $\pi_{1}$ and $\pi_{2}$ are transversal. Then two fibrations give the holomorphic coordinate $\left(z_{1}, z_{2}\right)$ of an appropriate neighbourhood $U_{x}$ of $x$, such that $\left\{z_{i}=a\right\}=\pi_{i}^{-1}(a) \cap U_{x}$.

For any $t_{i} \in \mathbb{P}^{1}$, let $X_{t_{i}}:=\pi_{i}^{-1}\left(t_{i}\right)$. If $t_{i}$ are close to $0,\left(E, \mathbf{F}, \mathbb{D}^{\lambda}\right)_{\mid X_{t_{i}}}$ are stable, and hence there exist tame harmonic metrics $h_{t_{i}}$ for $\left(E, \mathbf{F}, \mathbb{D}^{\lambda}\right){ }_{\mid X_{t_{i}}}$ such that $\operatorname{det}\left(h_{t_{i}}\right)=h_{\operatorname{det}(E) \mid X_{t_{i}}}$. Let $\theta_{t_{i}}$ denote the operator obtained from $\mathbb{D}_{\mid X_{t_{i}}}^{\lambda}$ and $h_{t_{i}}$ as in Section 2.2.1.

Let us take an appropriate neighbourhoods $B_{i} \subset \mathbb{P}^{1}$ of 0 . Recall Proposition 4.2. Then $\left\{h_{t_{1}} \mid t_{1} \in B_{1}\right\}$ are $C^{\infty}$-along $z_{2}$, and it is continuous with respect to $\left(z_{1}, z_{2}\right)$. The family $\left\{\theta_{t_{1}} \mid t_{1} \in B_{1}\right\}$ has a similar property. Thus, we obtain a continuous metric $h^{(1)}$ and the continuous section $\theta^{(1)}$ of $\operatorname{End}(E) \otimes \Omega^{1,0}$ around $x$. Similarly $\left\{h_{t_{2}} \mid t_{2} \in B_{2}\right\}$ is $C^{\infty}$ along $z_{1}$ and it is continuous with respect to $\left(z_{1}, z_{2}\right)$. The family $\left\{\theta_{t_{2}} \mid t_{2} \in B_{2}\right\}$ has a similar property. Thus, we obtain a continuous metric $h^{(2)}$ and the continuous section $\theta^{(2)}$ of $\operatorname{End}(E) \otimes \Omega^{1,0}$ around $x$.

We remark that $h^{(1)}=h_{\mathcal{V}}=h^{(2)}$ on $U_{x} \cap \mathcal{V}$ due to our construction of $h_{\mathcal{V}}$. Since $h^{(i)}$ are continuous, we obtain $h^{(1)}=h^{(2)}$ on $U_{x}$. Then we obtain that $h^{(i)}$ are $C^{1}$ on $U_{x}$, due to the continuity of $\theta^{(i)}$.

Therefore, we obtain the $C^{1}$-metric $h$ of $E$ on $X^{*}-Z$ with the following properties:

- $h_{\mid \mathcal{V}}=h_{\mathcal{V}}$

- For any $s \in \mathcal{U}$, we have $h_{\mid X_{s}^{*}}=h_{s}$ and $\theta_{h \mid X_{s}^{*}}=\theta_{h_{s}}$. 
5.2.6 Pluri-harmonicity We would like to show that $h$ is pluri-harmonic. By the formalism explained in Section 2.2.1, the operators $\bar{\partial}_{h}$ and $\theta_{h}$ are given on $X-(D \cup Z)$ from $h$ and $\mathbb{D}^{\lambda}$. Let us take any $C^{\infty}$ metric $h^{\prime}$ of $E$ on $X-D$, and let $s^{\prime}$ be the endomorphism determined by $h=h^{\prime} \cdot s^{\prime}$. Then $s^{\prime}$ is $C^{1}$, and we have the following relation:

$$
\bar{\partial}_{h}=\bar{\partial}_{h^{\prime}}+\frac{\lambda}{1+|\lambda|^{2}} s^{\prime-1} \delta_{h^{\prime}}^{\prime \prime} s^{\prime}, \quad \theta_{h}=\theta_{h^{\prime}}-\frac{\lambda}{1+|\lambda|^{2}} s^{\prime-1} \delta_{h^{\prime}}^{\prime} s^{\prime}
$$

Then we obtain $\bar{\partial}_{h} \theta_{h}$ as a distribution:

$$
\begin{aligned}
\bar{\partial}_{h} \theta_{h}=\bar{\partial}_{h^{\prime}} \theta_{h^{\prime}} & -\frac{\lambda}{1+|\lambda|^{2}} \bar{\partial}_{h^{\prime}}\left(s^{\prime-1} \delta_{h^{\prime}}^{\prime} s^{\prime}\right)+\frac{\lambda}{1+|\lambda|^{2}}\left[s^{\prime-1} \delta_{h^{\prime}}^{\prime \prime} s^{\prime}, \theta_{h^{\prime}}\right] \\
& -\left(\frac{\lambda^{2}}{1+|\lambda|^{2}}\right)^{2}\left[s^{\prime-1} \delta_{h^{\prime}}^{\prime \prime} s^{\prime}, s^{\prime-1} \delta_{h^{\prime}}^{\prime} s^{\prime}\right]
\end{aligned}
$$

Similarly, we obtain $G(h)$ as a distribution.

Lemma 5.12 $\bar{\partial}_{h} \theta_{h}=0$.

Proof For any point $x \in X^{*}-D$, let us take the holomorphic coordinate $\left(z_{1}, z_{2}\right)$ as before. We remark that the curves

$$
\left\{z_{i}=a\right\} \quad(i=1,2), \quad\left\{z_{1}+z_{2}=b\right\}, \quad\left\{z_{1}+\sqrt{-1} z_{2}=c\right\}
$$

can be regarded as parts of $X_{s^{\prime}}$ for some $s^{\prime} \in \mathcal{U}$. We have the expression $\theta=$ $f_{1} \cdot d z_{1}+f_{2} \cdot d z_{2}$, where $f_{i}$ are continuous sections of $\operatorname{End}(E)$. We have already known $\partial f_{1} / \partial \bar{z}_{1}=\partial f_{2} / \partial \bar{z}_{2}=0$. Thus, we have only to show $\partial f_{i} / \partial \bar{z}_{j}=0$ for $i \neq j$. Let us consider the change of the coordinate given by $w_{1}=z_{1}+z_{2}$ and $w_{2}=z_{1}-z_{2}$. Then we have the following:

$$
f_{1} \cdot d z_{1}+f_{2} \cdot d z_{2}=\frac{1}{2}\left(f_{1}+f_{2}\right) \cdot d w_{1}+\frac{1}{2}\left(f_{1}-f_{2}\right) \cdot d w_{2}
$$

Thus, we obtain the following:

$$
0=\frac{\partial}{\partial \bar{w}_{1}}\left(f_{1}+f_{2}\right)=\frac{1}{2}\left(\frac{\partial}{\partial \bar{z}_{1}}+\frac{\partial}{\partial \bar{z}_{2}}\right)\left(f_{1}+f_{2}\right)=\frac{1}{2}\left(\frac{\partial f_{2}}{\partial \bar{z}_{1}}+\frac{\partial f_{1}}{\partial \bar{z}_{2}}\right)
$$

Let us consider the change of the coordinate given by $u_{1}=z_{1}+\sqrt{-1} z_{2}$ and $u_{2}=$ $z_{1}-\sqrt{-1} z_{2}$. Then we have the following:

$$
f_{1} \cdot d z_{1}+f_{2} \cdot d z_{2}=\frac{1}{2}\left(f_{1}+\frac{1}{\sqrt{-1}} f_{2}\right) d u_{1}+\frac{1}{2}\left(f_{1}-\frac{1}{\sqrt{-1}} f_{2}\right) d u_{2}
$$


Thus, we obtain the following:

$$
\begin{aligned}
0=\frac{\partial}{\partial \bar{u}_{1}}\left(f_{1}+\frac{1}{\sqrt{-1}} f_{2}\right) & =\frac{1}{2}\left(\frac{\partial}{\partial \bar{z}_{1}}-\frac{1}{\sqrt{-1}} \frac{\partial}{\partial \bar{z}_{2}}\right)\left(f_{1}+\frac{1}{\sqrt{-1}} f_{2}\right) \\
& =\frac{1}{2}\left(\frac{1}{\sqrt{-1}} \frac{\partial f_{2}}{\partial \bar{z}_{1}}-\frac{1}{\sqrt{-1}} \frac{\partial f_{1}}{\partial \bar{z}_{2}}\right)
\end{aligned}
$$

From (63) and (64), we obtain $\partial f_{i} / \partial \bar{z}_{j}=0$ for $i \neq j$. Thus, we obtain $\bar{\partial}_{h} \theta_{h}=0$, and the proof of Lemma 5.12 is accomplished.

Lemma 5.13 $h$ is a harmonic metric for $\left(E, \mathbb{D}^{\lambda}\right)$ with respect to $\omega_{0}$ on $X^{*}-Z$. (Recall $Z=\left\{x \in X^{*} \mid \mathcal{U}_{x}=\varnothing\right\}$.)

Proof Due to Lemma 5.12, we have $\Lambda_{\omega} G(h)=\Lambda_{\omega}\left(\bar{\partial}_{h} \theta_{h}\right)=0$. Hence, we have only to show that $h$ is $C^{\infty}$. We obtain the following formula in the level of distribution, by the formalism explained in Section 2.2.5:

$$
\Delta_{h^{\prime}, \omega}^{\lambda}\left(s^{\prime}\right)=s^{\prime}\left(-\Lambda_{\omega} G\left(h^{\prime}\right)\right)+\sqrt{-1} \Lambda_{\omega} \mathbb{D}^{\lambda} s^{\prime} \cdot s^{\prime-1} \cdot \mathbb{D}_{h^{\prime}}^{\lambda \star} s^{\prime}
$$

The right hand side is $C^{0}$. Hence, by using the elliptic regularity and the standard bootstrapping, we obtain that $s^{\prime}$ is $C^{\infty}$. Thus, we obtain Lemma 5.13.

Lemma 5.14 $h$ is pluri-harmonic metric of $E_{\mid X^{*}-Z}$.

Proof We have already shown $\bar{\partial}_{h} \theta_{h}=0$ in Lemma 5.12. According to Corollary 2.30, we have only to show $\theta_{h}^{2}=0$. Due to Corollary 5.7 and $\theta_{h \mid X_{s}}=\theta_{s}$, we know that the sequence $\left\{\theta^{(\epsilon)}\right\}$ converges to $\theta_{h}$ almost everywhere. In particular, we obtain the almost everywhere convergence of $\left\{\theta^{(\epsilon) 2}\right\}$ to $\theta_{h}^{2}$. On the other hand, we know the almost everywhere convergence $G\left(h^{(\epsilon)}\right) \longrightarrow 0$, due to (60). We have $G\left(h^{(\epsilon)}\right)=\bar{\partial}^{(\epsilon) 2}+\bar{\partial}^{(\epsilon)} \theta^{(\epsilon)}+\theta^{(\epsilon) 2}$, which is the decomposition into $(2,0),(1,1)$ and $(0,2)$-forms. Therefore, we obtain $\theta_{h}^{2}=0$, almost everywhere. Thus, we obtain Lemma 5.14 .

Lemma 5.15 $h$ gives a pluri-harmonic metric of $E_{\mid X^{*}}$.

Proof We have only to check that $h$ gives a $C^{\infty}$-metric of $E_{\mid X^{*}}$. Let $Q$ be a point of $Z$. Let $\left(U, z_{1}, z_{2}\right)$ be a holomorphic coordinate around $Q$ such that $z_{1}(Q)=z_{2}(Q)=$ 0 . The pluri-harmonic metric $h$ of $\left(E, \mathbb{D}^{\lambda}\right)_{\mid U-\{Q\}}$ is given. We would like to show that $h$ is naturally extended to the pluri-harmonic metric of $\left(E, \mathbb{D}^{\lambda}\right)_{\mid U}$.

We have $\theta=f_{1} \cdot d z_{1}+f_{2} \cdot d z_{2}$ defined on $U-\{Q\}$. Let us consider the characteristic polynomials $\operatorname{det}\left(t-f_{i}\right)$ for $i=1,2$. The coefficients are holomorphic on $U-\{Q\}$, and 
thus on $U$ due to the theorem of Hartog's. Hence, the eigenvalues of $f_{i}$ are bounded on $U$. Let us consider the restriction of $\left(E, \mathbb{D}^{\lambda}, h\right)$ to the $\operatorname{discs} C\left(a_{j}\right):=\left\{z_{j}=a_{j}\right\}$ $\left(a_{j} \neq 0\right)$ for $j=1,2$. Then it can be shown that the norms $\left|f_{i \mid C\left(a_{j}\right)}\right|_{h} \leq C(i \neq j)$ can be dominated independently from $a_{j}$. (See Lemma 2.7 in [19], for example.) Thus, $f_{i}$ are bounded with respect to $h$ on $U-\{Q\}$. In other words, $\theta$ is bounded on $U-\{Q\}$.

Let $E^{\prime}:=E_{\mid U-\left\{z_{1} \cdot z_{2}=0\right\}}$. Let us consider the sheaf ${ }^{\diamond} E^{\prime}$ on $U$ of sections $g$ satisfying the growth condition $|g|_{h}=O\left(\prod\left|z_{i}\right|^{-\epsilon}\right.$ ) for any $\epsilon>0$ (Section 2.6.3). By using the result of the asymptotic behaviour of tame harmonic bundle at $\lambda[15],{ }^{\diamond} E^{\prime}$ is locally free on $U$. Since ${ }^{\diamond} E^{\prime}$ and $E_{\mid U-\{Q\}}$ are naturally isomorphic on $U-\{Q\}$, they are isomorphic on $U$. Let $h^{\prime}$ be any $C^{\infty}$-metric of $E_{\mid U}$, and let $s^{\prime}$ be the endomorphism determined by $h=h^{\prime} \cdot s^{\prime}$. Due to the norm estimate given in [15], the metrics $h$ and $h^{\prime}$ are mutually bounded. Hence, $s^{\prime}$ and $\left(s^{\prime}\right)^{-1}$ are bounded on $U$. Let $\delta_{h^{\prime}}^{\prime}$ and $\delta_{h^{\prime}}^{\prime \prime}$ be obtained from $\mathbb{D}^{\lambda}$ and $h^{\prime}$ as in Section 2.2.1. Due to the boundedness of $\theta$, we have the boundedness of $\left(s^{\prime}\right)^{-1} \delta_{h^{\prime}}^{\prime} s^{\prime}$ on $U-\{Q\}$. Due to the boundedness of $\theta^{\dagger}$, we have the boundedness of $\left(s^{\prime}\right)^{-1} \delta_{h^{\prime}}^{\prime \prime} s^{\prime}$ on $U-\{Q\}$. Then we can deduce that $s^{\prime-1} \mathbb{D}^{\lambda} s^{\prime}$ is also bounded on $U-\{Q\}$. (See Section 2.2.5. for example.) Since we have the formula $\Delta_{h^{\prime}, \omega_{0}}^{\lambda} s^{\prime}=s^{\prime}\left(-\Lambda_{\omega_{0}} G\left(h^{\prime}\right)\right)+\Lambda_{\omega_{0}} \mathbb{D}_{h^{\prime}}^{\lambda} s^{\prime} \cdot s^{\prime-1} \cdot \mathbb{D}_{h^{\prime}}^{\lambda \star} s^{\prime}$, we can conclude that $s^{\prime}$ is $C^{\infty}$ due to the standard bootstrapping. Namely, $h$ is extended to the $C^{\infty}$-metric of $E_{\mid U}$.

5.2.7 The end of the proof of Theorem 5.5 Now, we have only to show that $h$ is tame and adapted to the parabolic structure. Since $h_{\mid X_{s}}=h_{s}$ for any $s \in \mathcal{U}$, the tameness immediately follows from the curve test. (See Proposition 2.52.) Hence, we obtain the harmonic bundle $\left(\left(E, \mathbb{D}^{\lambda}\right)_{\mid X^{*}}, h\right)$. We have the locally free sheaf $\widetilde{E}:={ }_{\mathbf{c}}\left(E_{\mid X^{*}}\right)$ with the induced parabolic structure $\mathbf{F}$ (Section 2.6.3). We would like to show that $\left(E, \mathbf{F}, \mathbb{D}^{\lambda}\right)$ and $\left(\widetilde{E}, \mathbf{F}, \mathbb{D}^{\lambda}\right)$ are isomorphic. For that purpose, we see that the identity $E_{\mid X^{*}} \longrightarrow E_{\mid X^{*}}$ can be prolonged to the homomorphism $\Psi: E \longrightarrow \widetilde{E}$. Let $Q$ be any smooth point of $D_{i} \subset D$. We take a holomorphic coordinate $\left(U_{Q}, z_{1}, z_{2}\right)$ with the following properties:

- The curve $z_{1}^{-1}(0)$ is the same as $U_{Q} \cap D$.

- The curves $C(b):=z_{2}^{-1}(b)$ are parts of $X_{s(b)}$ for $s(b) \in \mathcal{U}$.

Let $f$ be a holomorphic section of $E_{\mid U}$. Let $c_{i}$ be the $i$-th component of c. Since the restriction $h_{\mid X_{s(b)}}$ is the same as $h_{s(b)}$, we have $\left|f_{\mid C(b)}\right|_{h}=O\left(\left|z_{1}\right|^{-c_{i}-\epsilon}\right)$ for any $\epsilon>0$. Then we obtain $|f|_{h}=O\left(\left|z_{1}\right|^{-c_{i}-\epsilon}\right)$ for any $\epsilon>0$, due to the result given in [15]. Thus, $f$ naturally gives the section of $\tilde{E}$ on $U$. Therefore, we obtain the 
morphism $E \longrightarrow \widetilde{E}$ on $X-\left(\cup_{i \neq j} D_{i} \cap D_{j}\right)$. It is naturally extended to the morphism $E \longrightarrow \widetilde{E}$.

Recall that the restriction of $\widetilde{E}={ }_{\mathbf{c}} E(h)$ to $X_{S}$ is the same as $\mathbf{c}\left(E_{\mid X_{s}^{*}}\right)\left(h_{s}\right)$. (See Corollary 8.52 of [15].) Therefore, the restrictions of $\Psi$ to $X_{S}$ are isomorphic, by construction. Hence, $\Psi$ is isomorphic on $X-\left(\bigcup_{i \neq j} D_{i} \cap D_{j}\right)$, and thus on $X$. By a similar argument, we can show that the parabolic structures are also the same. Thus, the proof of Theorem 5.5 is finished.

\subsection{Correspondences}

\subsubsection{Kobayashi-Hitchin correspondence in the higher dimensional case Let $X$} be an $n$-dimensional smooth irreducible projective variety with an ample line bundle $L$, and let $D$ be a simple normal crossing hypersurface with the irreducible decomposition $D=\bigcup_{i \in S} D_{i}$. Let $\left(\mathbf{E}_{*}, \mathbb{D}^{\lambda}\right)$ be a $\mu_{L}$-stable regular filtered $\lambda$-flat bundle on $(X, D)$ in codimension two with trivial characteristic numbers

$$
\operatorname{par}-\operatorname{deg}_{L}\left(\mathbf{E}_{*}\right)=\int_{X} \operatorname{par}^{-c_{2, L}}\left(\mathbf{E}_{*}\right)=0
$$

We put $\left(E, \mathbb{D}^{\lambda}\right):=\left(\mathbf{E}_{*}, \mathbb{D}^{\lambda}\right)_{\mid X-D}$. Recall par-c ${ }_{1}\left(\mathbf{E}_{*}\right)=0$ due to the BogomolovGieseker inequality, the Hodge index theorem and a theorem of Lefschetz. For each $\mathbf{c} \in \mathbf{R}^{S}$, we have the determinant line bundle $\operatorname{det}\left({ }_{\mathbf{c}} E\right)$ of torsion-free sheaf $\mathbf{c} E$, on which we have the induced parabolic structure and the induced flat $\lambda$-connection. Thus, we obtain the canonically determined regular filtered $\lambda$-flat bundle $\left(\operatorname{det} \mathbf{E}_{*}, \mathbb{D}^{\lambda}\right)$ on $(X, D)$ of rank one. We also have par-c $\operatorname{c}_{1}\left(\operatorname{det} \mathbf{E}_{*}\right)=\operatorname{par}-\mathrm{c}_{1}\left(\mathbf{E}_{*}\right)=0$. Therefore, we can take a pluri-harmonic metric $h_{\operatorname{det} E}$ of $\left(\operatorname{det}(E), \mathbb{D}^{\lambda}\right)$ which is adapted to the parabolic structure of $\operatorname{det} \mathbf{E}_{*}$ by using classical Hodge-de Rham theory. (See Lemma 2.26, for example.) By the assumption, we have a subset $Z \subset D$ with $\operatorname{codim}_{X}(Z) \geq 3$ such that $\left(\mathbf{E}_{*}, \mathbb{D}^{\lambda}\right)_{\mid X-Z}$ is a regular filtered $\lambda$-flat bundle.

Theorem 5.16 There exists the unique tame pluri-harmonic metric $h$ of $\left(E, \mathbb{D}^{\lambda}\right)$ with the following properties:

- $\operatorname{det}(h)=h_{\operatorname{det} E}$.

- It is adapted to the parabolic structure of $\mathbf{E}_{*}$ on $X-Z$. Namely,

$$
\left(\mathbf{E}_{*}(h), \mathbb{D}^{\lambda}\right)_{\mid X-Z} \simeq\left(\mathbf{E}_{*}, \mathbb{D}^{\lambda}\right)_{\mid X-Z},
$$

where $\left(\mathbf{E}_{*}(h), \mathbb{D}^{\lambda}\right)$ denotes the regular filtered $\lambda$-flat bundle on $(X, D)$ obtained from $\left(E, \mathbb{D}^{\lambda}, h\right)$. (See Section 2.6.) 
Proof Due to Mehta-Ramanathan type theorem (Proposition 2.21), the uniqueness can be easily reduced to the $\operatorname{dim} X=1$ case, by considering the restriction to the generic curves $C \subset X$. We have already known it (Proposition 2.56).

We will use the induction on the dimension $n$ to show the existence. The case $n=2$ has already been shown (Theorem 5.5). Assume that $L^{m}$ is sufficiently ample. We put $\mathbb{P}_{m}:=\mathbb{P}\left(H^{0}\left(X, L^{m}\right)^{\vee}\right)$. For any $s \in \mathbb{P}_{m}$, we put $X_{s}:=s^{-1}(0)$. Recall Proposition 2.21. Let $\mathcal{U}$ be the nonempty Zariski open subset of $\mathbb{P}_{m}$ which consists of $s \in \mathbb{P}_{m}$ with the following properties:

- $X_{S}$ is smooth, and $D_{S}:=X_{S} \cap D$ is a normal crossing divisor.

- The codimension of $Z \cap X_{s}$ in $X_{S}$ is larger than 3 .

- $\left(\mathbf{E}, \mathbb{D}^{\lambda}\right)_{\mid X_{s}}$ is $\mu_{L}$-stable.

We use the existence hypothesis in the $(n-1)$-dimensional case of the induction. Then we may have the tame pluri-harmonic metric $h_{s}$ of $\left(E, \mathbb{D}^{\lambda}\right)_{\mid X_{s} \backslash D}$ with $\operatorname{det}\left(h_{s}\right)=$ $h_{\operatorname{det} E \mid X_{s} \backslash D}$ which is adapted to the parabolic structure on $X_{s} \backslash W$. We also use the uniqueness result in the $(n-2)$-dimensional case. Then we can show the existence of a finite subset $Z^{\prime} \subset X-D$ and a metric $h$ of $E_{\mid X-D}$ such that $h_{s \mid P}=h_{\mid P}$. By the arguments given in Sections 5.2.5-5.2.7, we can show that $h$ is the desired metric. The only different point is the argument to show the vanishing of $G(h)=0$. Due to $\operatorname{dim} X_{s} \geq 2$, it can be shown more easily.

Theorem 5.17 Let $\left(\mathbf{E}_{*}, \mathbb{D}^{\lambda}\right)$ be a saturated $\mu_{L}$-stable regular filtered $\lambda$-flat sheaf on $(X, D)$ with the trivial characteristic numbers par- $\operatorname{deg}_{L}\left(\mathbf{E}_{*}\right)=\int_{X} \operatorname{par}_{-c_{2, L}}\left(\mathbf{E}_{*}\right)=0$. We put $\left(E, \mathbb{D}^{\lambda}\right):=\left(\mathbf{E}_{*}, \mathbb{D}^{\lambda}\right)_{\mid X-D}$. Then there exists a pluri-harmonic metric $h$ of $\left(E, \mathbb{D}^{\lambda}\right)$ such that the induced regular filtered $\lambda$-flat bundle $\left(\mathbf{E}_{*}(h), \mathbb{D}^{\lambda}\right)$ is isomorphic to $\left(\mathbf{E}_{*}, \mathbb{D}^{\lambda}\right)$. (See Proposition 2.53 for an induced regular filtered $\lambda$-flat bundle.) Such a metric is unique up to positive constant multiplications. In particular, $\mathbf{E}_{*}$ is a filtered bundle.

Proof Since a saturated regular filtered $\lambda$-flat sheaf is a regular filtered $\lambda$-flat bundle in codimension two (Lemma 2.9), we may apply Theorem 5.16. Then there exists a pluri-harmonic metric $h$ and a subset $W \subset D$ with $\operatorname{codim}_{X}(W) \geq 3$ such that the induced regular filtered $\lambda$-flat bundle $\left(\mathbf{E}_{*}(h), \mathbb{D}^{\lambda}\right)$ is isomorphic to $\left(\mathbf{E}_{*}, \mathbb{D}^{\lambda}\right)$ on $X-W$. Since both of $\left(\mathbf{E}_{*}(h), \mathbb{D}^{\lambda}\right)$ and $\left(\mathbf{E}_{*}, \mathbb{D}^{\lambda}\right)$ are saturated, they are isomorphic on $X$. 
5.3.2 An equivalence of some categories We say that a $\mu_{L}$-polystable regular filtered $\lambda$-flat bundle $\left(\mathbf{E}_{*}, \mathbb{D}^{\lambda}\right)$ has trivial characteristic numbers, if each $\mu_{L}$-stable direct summand $\left(\mathbf{E}_{1 *}, \mathbb{D}_{1}^{\lambda}\right)$ satisfy par-deg ${ }_{L}\left(\mathbf{E}_{1 *}\right)=\int_{X} \operatorname{par}^{-c_{2, L}}\left(\mathbf{E}_{1 *}\right)=0$. Let $\mathcal{C}_{\lambda}^{\text {poly }}$ denote the category of $\mu_{L}$-polystable regular filtered $\lambda$-flat bundles $\left(\mathbf{E}_{*}, \mathbb{D}^{\lambda}\right)$ on $(X, D)$ with trivial characteristic numbers. Morphisms $f:\left(\mathbf{E}_{1 *}, \mathbb{D}_{1}^{\lambda}\right) \longrightarrow\left(\mathbf{E}_{2 *}, \mathbb{D}_{2}^{\lambda}\right)$ are defined to be an $\mathcal{O}_{X}$-homomorphism $f: \mathbf{E}_{1} \longrightarrow \mathbf{E}_{2}$ satisfying $\mathbb{D}_{2}^{\lambda} \circ f=f \circ \mathbb{D}_{1}^{\lambda}$ and $f\left({ }_{\mathbf{c}} E_{1}\right) \subset{ }_{\mathbf{c}} E_{2}$ for any c. By Lemma 2.12 and Corollary 2.14 , the category $\mathcal{C}_{\lambda}^{\text {poly }}$ is semisimple, ie, any object is a direct sum of simple objects.

Corollary 5.18 Let $\lambda_{i}(i=1,2)$ be two complex numbers. We have a functor $\Xi_{\lambda_{1}, \lambda_{2}}: \mathcal{C}_{\lambda_{1}}^{\text {poly }} \longrightarrow \mathcal{C}_{\lambda_{2}}^{\text {poly }}$, which gives an equivalence of the categories. (See the proof for the construction.) It preserves direct sums, tensor products and duals.

Proof Let $\left(\mathbf{E}_{*}^{\lambda_{1}}, \mathbb{D}^{\lambda_{1}}\right)$ be an object of $\mathcal{C}_{\lambda_{1}}^{\text {poly }}$. We put $E^{\lambda_{1}}:=\mathbf{E}_{\mid D}^{\lambda_{1}}$. We have a pluri-harmonic metric $h$ of $\left(E^{\lambda_{1}}, \mathbb{D}^{\lambda_{1}}\right)$, which is adapted to the parabolic structure. Then we obtain the operators $\bar{\partial}_{h}, \partial_{h}, \theta_{h}, \theta_{h}^{\dagger}$, as in Section 2.2.1. Note that the holomorphic structure of $E^{\lambda_{1}}$ is given by $\bar{\partial}_{h}+\lambda_{1} \theta_{h}^{\dagger}$. The $(0,1)$-operator $\bar{\partial}_{h}+\lambda_{2} \theta_{h}^{\dagger}$ also gives a holomorphic structure of $C^{\infty}$-bundle $E^{\lambda_{1}}$. To distinguish them, we use the notation $E^{\lambda_{2}}$, when we consider the holomorphic structure $\bar{\partial}_{h}+\lambda_{2} \theta_{h}^{\dagger}$. We put $\mathbb{D}^{\lambda_{2}}:=\bar{\partial}_{h}+\theta_{h}+\lambda_{2}\left(\partial_{h}+\theta_{h}^{\dagger}\right)$, which gives a flat $\lambda_{2}$-connection of $E^{\lambda_{2}}$. The metric $h$ is pluri-harmonic for $\left(E^{\lambda_{2}}, \mathbb{D}^{\lambda_{2}}\right)$. Since the corresponding Higgs bundle for $\left(E^{\lambda_{1}}, \mathbb{D}^{\lambda_{1}}, h\right)$ and $\left(E^{\lambda_{2}}, \mathbb{D}^{\lambda_{2}}, h\right)$ are the same, we obtain the tameness of $\left(E^{\lambda_{2}}, \mathbb{D}^{\lambda_{2}}, h\right)$. Therefore, we obtain the prolongment $\left(\mathbf{E}_{*}^{\lambda_{2}}, \mathbb{D}^{\lambda_{2}}\right)$, which are $\mu_{L^{-}}$ polystable regular filtered $\lambda_{2}$-flat bundle on $(X, D)$ with trivial characteristic numbers (Proposition 2.55).

We remark that $\left(\mathbf{E}_{*}^{\lambda_{2}}, \mathbb{D}^{\lambda_{2}}\right)$ is independent of the choice of $h$, due to the uniqueness in Proposition 2.56. Therefore, we put $\Xi_{\lambda_{1}, \lambda_{2}}\left(\mathbf{E}^{\lambda_{1}}, \mathbb{D}^{\lambda_{1}}\right):=\left(\mathbf{E}^{\lambda_{2}}, \mathbb{D}^{\lambda_{2}}\right)$. It is easy to see that $\Xi_{\lambda_{1}, \lambda_{2}}$ gives a functor. (Note that the categories are semisimple.) It is also easy to see that $\Xi_{\lambda_{2}, \lambda_{1}} \circ \Xi_{\lambda_{1}, \lambda_{2}}\left(\mathbf{E}^{\lambda_{1}}, \mathbb{D}^{\lambda_{1}}\right)$ is naturally isomorphic to $\left(\mathbf{E}^{\lambda_{1}}, \mathbb{D}^{\lambda_{1}}\right)$. The compatibility with the direct sums, duals and tensor products are obtained from the corresponding compatibility of the prolongments for tame harmonic bundles [15]. $\square$

5.3.3 Corlette-Jost-Zuo metric Let $(E, \nabla)$ be a simple flat bundle on $X-D$, ie, the associated representation of the fundamental group of $X-D$ is assumed to be simple. Recall that there exists a Corlette-Jost-Zuo metric of $(E, \nabla)$ which is a pure imaginary tame pluri-harmonic metric. (See Corlette [2] for the case $D=\varnothing$ and Jost and Zuo [10] for the general case. See also Mochizuki [15].) Let us show the existence theorem for Corlette-Jost-Zuo metric from the viewpoint of the Kobayashi-Hitchin correspondence. 
Let $(\widetilde{E}, \nabla)$ be the Deligne extension. It is equipped with the canonical parabolic structure $\mathbf{F}$ determined by the condition $a+\operatorname{Re}(\alpha)=0$ for any $(a, \alpha) \in \mathcal{K} \mathcal{M S}(\widetilde{E}, \mathbf{F})$. Let $L$ be any ample line bundle on $X$. As remarked in Corollary 3.25, we have the vanishings

$$
\operatorname{par}_{-d^{\prime}}(\widetilde{E}, \mathbf{F})=0, \quad \int_{X} \operatorname{par}^{-c_{2, L}}(\widetilde{E}, \mathbf{F})=0 .
$$

Simplicity of $(E, \nabla)$ is equivalent to $\mu_{L}$-stability of $(\widetilde{E}, \mathbf{F})$, which follows from the fact that the first formula in (65) also holds for any flat subbundle of $(E, \nabla)$. Hence, we have a tame pluri-harmonic metric $h$ of $(E, \nabla)$, adapted to $\mathbf{F}$. It is unique up to positive constant multiplication. Let $\left(\mathcal{E}_{*}^{\lambda}, \mathbb{D}^{\lambda}\right)$ denote the regular filtered $\lambda$-flat bundle associated to the tame harmonic bundle $(E, \nabla, h)$. By construction, $(\widetilde{E}, \mathbf{F})$ is the same as ${ }^{\diamond} \mathcal{E}^{1}$ with the induced parabolic structure. Recall the correspondence of KMS-spectrum due to Simpson [18]:

$$
\mathcal{K} \mathcal{M S}\left(\mathcal{E}^{0}, i\right) \longleftrightarrow \mathcal{K} \mathcal{M S}\left(\mathcal{E}^{1}, i\right), \quad(b, \beta) \longleftrightarrow(b+2 \operatorname{Re}(\beta), \beta-b-\bar{\beta})
$$

Hence, we can check $\mathcal{K} \mathcal{M S}\left(\mathcal{E}^{0}, i\right) \subset \mathbf{R} \times(\sqrt{-1} \mathbf{R})$, ie, the eigen values of the residues of the Higgs field is purely imaginary. It means that $h$ is a Corlette-Jost-Zuo metric.

\section{Filtered local system}

We established the correspondence between regular filtered flat bundles and regular filtered Higgs bundles which are $\mu_{L}$-stable with trivial characteristic numbers. One more important piece in the nonabelian Hodge theory is filtered local system, which is the topic in this section.

In Section 6.1, we define filtered local system which is a straightforward generalization of Simpson's definition in the one dimensional case. In Section 6.2, we construct a functor which gives an equivalence of the categories of filtered local systems and saturated regular filtered $\lambda$-flat sheaves. We show that it preserves parabolic characteristic numbers and stability conditions.

Note that filtered bundle corresponds to filtered local system which satisfies some compatibility condition around the intersection of the divisors, like a locally abelian condition in [9].

\subsection{Definition}

6.1.1 Filtered structure Let $X$ be a complex manifold, and let $D$ be a simple normal crossing hypersurface with the irreducible decomposition $D=\bigcup_{i \in S} D_{i}$. We 
set $D^{[2]}:=\bigcup_{i \neq j} D_{i} \cap D_{j}$ and $D_{i}^{\circ}:=D_{i} \backslash \bigcup_{j \neq i} D_{j}$. Let $\mathcal{L}$ be a local system on $X-D$. A filtered structure of $\mathcal{L}$ at $D$ is a tuple of increasing filtrations ${ }^{i} \mathcal{F}(i \in S)$ of $\mathcal{L}_{\mid U_{i} \backslash D}$ indexed by $\mathbf{R}$, where $U_{i}$ denotes an appropriate open neighbourhood of $D_{i}$. Let $U_{i}^{\prime}$ be an open neighbourhood of $D_{i}$ such that $U_{i}^{\prime} \subset U_{i}$, then we have the induced filtration ${ }^{i} \mathcal{F}_{\mid U_{i}^{\prime} \backslash D}$, and the filtration ${ }^{i} \mathcal{F}$ can be reconstructed from ${ }^{i} \mathcal{F}_{\mid U_{i}^{\prime} \backslash D}$. Hence, we define two filtered structures $\left({ }^{i} \mathcal{F}, U_{i} \mid i \in S\right)$ and $\left({ }^{i} \mathcal{F}^{\prime}, U_{i}^{\prime} \mid i \in S\right)$ are equivalent, if there exists an open neighbourhood $U_{i}^{\prime \prime}$ of $D_{i}$ such that $U_{i}^{\prime \prime} \subset U_{i} \cap U_{i}^{\prime}$ and ${ }^{i} \mathcal{F}_{\mid U_{i}^{\prime \prime}}={ }^{i} \mathcal{F}_{\mid U_{i}^{\prime \prime}}^{\prime}$. A local system $\mathcal{L}$ equipped with an equivalence class of filtered structures $\left({ }^{i} \mathcal{F}, U_{i}\right)$ is called a filtered local system, and it is denoted by $\mathcal{L}_{*}$. We do not have to care about the choice of open neighbourhoods $U_{i}$.

Morphisms of filtered local systems $f: \mathcal{L}_{1 *} \longrightarrow \mathcal{L}_{2 *}$ are defined to be a morphism $f: \mathcal{L}_{1} \longrightarrow \mathcal{L}_{2}$ of local systems preserving the filtered structures in an obvious sense. We denote by $\tilde{\mathcal{C}}(X, D)$ the category of filtered local systems on $(X, D)$.

\subsubsection{Characteristic numbers We put}

and

$$
\begin{aligned}
U_{i}^{*} & :=U_{i} \backslash D \\
{ }^{i} \operatorname{Gr}_{a}{ }^{\mathcal{F}}\left(\mathcal{L}_{\mid U_{i}^{*}}\right) & :={ }^{i} \mathcal{F}_{a}\left(\mathcal{L}_{\mid U_{i}^{*}}\right) /{ }^{i} \mathcal{F}_{<a}\left(\mathcal{L}_{\mid U_{i}^{*}}\right)
\end{aligned}
$$

Since the local monodromy around $D_{i}$ preserves the filtration ${ }^{i} \mathcal{F}$, we obtain the induced endomorphism of ${ }^{i} \mathrm{Gr}_{a}^{\mathcal{F}}\left(\mathcal{L}_{\mid U_{i}^{*}}\right)$, and thus the generalized eigen decomposition:

$$
{ }^{i} \mathrm{Gr}_{a}^{\mathcal{F}}\left(\mathcal{L}_{\mid U_{i}^{*}}\right)=\bigoplus_{\omega}^{i} \operatorname{Gr}_{(a, \omega)}^{\mathcal{F}, \mathbb{E}}\left(\mathcal{L}_{\mid U_{i}^{*}}\right)
$$

We consider the following sets:

$$
\begin{aligned}
\operatorname{Par}\left(\mathcal{L}_{*}, i\right) & :=\left\{a \in \mathbf{R} \mid{ }^{i} \operatorname{Gr}_{a}^{\mathcal{F}}\left(\mathcal{L}_{\mid U_{i}^{*}}\right) \neq 0\right\} \\
\mathcal{K} \mathcal{M S}\left(\mathcal{L}_{*}, i\right) & :=\left\{(a, \omega) \in \mathbf{R} \times \mathbf{C}^{*} \mid{ }^{i} \operatorname{Gr}_{(a, \omega)}^{\mathcal{F}, \mathbb{E}}\left(\mathcal{L}_{\mid U_{i}^{*}}\right) \neq 0\right\}
\end{aligned}
$$

The parabolic first Chern class is defined as follows:

$$
\begin{aligned}
\operatorname{wt}\left(\mathcal{L}_{*}, i\right) & :=\sum_{a \in \mathcal{P a r}\left(\mathcal{L}_{*}, i\right)} a \cdot \operatorname{rank}^{i} \operatorname{Gr}_{a}^{\mathcal{F}}\left(\mathcal{L}_{\mid U_{i}^{*}}\right) \\
\operatorname{par}_{-c_{1}}\left(\mathcal{L}_{*}\right) & :=-\sum_{i \in S} \operatorname{wt}\left(\mathcal{L}_{*}, i\right) \cdot\left[D_{i}\right] \in H^{2}(X, \mathbf{R})
\end{aligned}
$$

Here $\left[D_{i}\right]$ denotes the cohomology class of $D_{i}$.

Let $\operatorname{Irr}\left(D_{i} \cap D_{j}\right)$ denote the set of the irreducible components of $D_{i} \cap D_{j}$. For each $P \in \operatorname{Irr}\left(D_{i} \cap D_{j}\right)$, let $U_{P}$ be an appropriate open neighbourhood of $P$ in $X$ such that 
$U_{P} \subset U_{i} \cap U_{j}$. We put $U_{P}^{*}:=U_{P} \backslash D$. We have the two filtrations ${ }^{i} \mathcal{F}$ and ${ }^{j} \mathcal{F}$ of $\mathcal{L}_{\mid U_{P}^{*}}$. The naturally induced graded local system is denoted as follows:

$$
\begin{aligned}
{ }^{P} \operatorname{Gr}_{\left(a_{i}, a_{j}\right)}^{\mathcal{F}}\left(\mathcal{L}_{\left.\mid U_{P}^{*}\right)}:=\right. & \frac{{ }^{i} \mathcal{F}_{a_{i}} \cap{ }^{j} \mathcal{F}_{a_{j}}}{\sum_{\left(b_{i}, b_{j}\right) \lesseqgtr\left(a_{i}, a_{j}\right)}{ }^{i} \mathcal{F}_{b_{i}} \cap{ }^{j} \mathcal{F}_{b_{j}}} \\
{ }^{P} \mathrm{Gr}^{\mathcal{F}}\left(\mathcal{L}_{\mid U_{P}^{*}}\right) & =\bigoplus_{\left(a_{i}, a_{j}\right) \in \mathbf{R}^{2}}{ }^{{ }^{2}} \mathrm{Gr}_{\left(a_{i}, a_{j}\right)}\left(\mathcal{L}_{\mid U_{P}^{*}}\right)
\end{aligned}
$$

Here $\left(b_{i}, b_{j}\right) \Varangle\left(a_{i}, a_{j}\right)$ means " $b_{i} \leq a_{i}, b_{j} \leq a_{j}$ and $\left(b_{i}, b_{j}\right) \neq\left(a_{i}, a_{j}\right)$ ". We have the two endomorphisms induced by the local monodromies around $U_{P} \cap D_{i}$ and $U_{P} \cap D_{j}$, which are commutative. Hence, we obtain the generalized eigen decomposition:

$$
{ }^{P} \operatorname{Gr}_{\mathbf{a}}^{\mathcal{F}}\left(\mathcal{L}_{\mid U_{P}^{*}}\right)=\bigoplus_{\boldsymbol{\omega} \in\left(\mathbf{C}^{*}\right)^{2}}{ }^{P} \operatorname{Gr}_{\mathbf{a}, \boldsymbol{\omega}}^{\mathcal{F}, \mathbb{E}}\left(\mathcal{L}_{\mid U_{P}^{*}}\right)
$$

The sets $\operatorname{Par}\left(\mathcal{L}_{*}, P\right)$ and $\mathcal{K} \mathcal{M S}\left(\mathcal{L}_{*}, P\right)$ are defined as follows:

$$
\begin{gathered}
\operatorname{Par}\left(\mathcal{L}_{*}, P\right):=\left\{\left(a_{i}, a_{j}\right) \in \mathbf{R}^{2} \mid{ }^{P} \mathrm{Gr}_{\left(a_{i}, a_{j}\right)}^{\mathcal{F}}\left(\mathcal{L}_{\mid U_{P}^{*}}\right) \neq 0\right\} \\
\mathcal{K} \mathcal{M S}\left(\mathcal{L}_{*}, P\right):=\left\{(\mathbf{a}, \boldsymbol{\omega}) \in \mathbf{R}^{2} \times\left(\mathbf{C}^{*}\right)^{2} \mid{ }^{P}{ }^{\mathcal{G r}}{ }_{(\mathbf{a}, \boldsymbol{\omega})}^{\mathcal{F}, \mathbb{E}}\left(\mathcal{L}_{\mid U_{P}^{*}}\right) \neq 0\right\}
\end{gathered}
$$

The parabolic second Chern character is defined as follows:

$$
\begin{aligned}
\operatorname{par}-\operatorname{ch}_{2}\left(\mathcal{L}_{*}\right) & :=\frac{1}{2} \sum_{i \in S} \sum_{a \in \mathcal{P a r}\left(\mathcal{L}_{*}, i\right)} a^{2} \cdot \operatorname{rank}^{i} \operatorname{Gr}_{a}^{\mathcal{F}}(\mathcal{L}) \cdot\left[D_{i}\right]^{2} \\
+ & \frac{1}{2} \sum_{i \in S} \sum_{j \neq i} \sum_{P \in \operatorname{Irr}\left(D_{i} \cap D_{j}\right)} \sum_{\left(a_{i}, a_{j}\right) \in \mathcal{P} a r} a_{\left.\mathcal{L}_{*}, P\right)} \cdot a_{j} \cdot \operatorname{rank}^{P} \operatorname{Gr}_{\left(a_{i}, a_{j}\right)}^{\mathcal{F}}\left(\mathcal{L}_{\mid U_{P}^{*}}\right) \cdot[P]
\end{aligned}
$$

If $X$ is a smooth projective variety with an ample line bundle $L$, we set

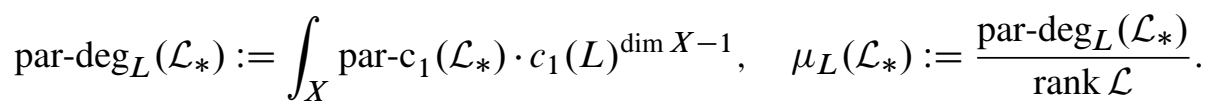

Then the notion of $\mu_{L}$-stability, $\mu_{L}$-semistability, and $\mu_{L}$-polystability for filtered local systems on $(X, D)$ are defined in the standard manner. We also put

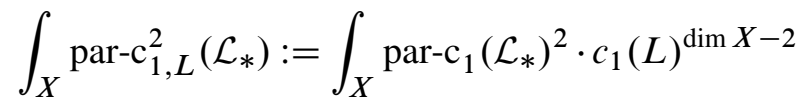

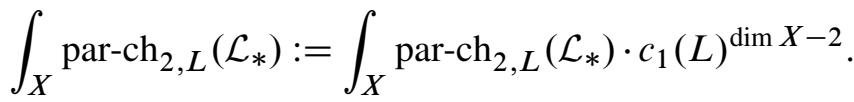




\subsection{Correspondence}

In this subsection, we explain the correspondence of filtered local systems on $(X, D)$ and saturated regular filtered $\lambda$-flat sheaves $(\lambda \neq 0)$ as in Proposition 1.8. See Section 2.1.3 for saturated regular filtered $\lambda$-flat sheaves. Since we have the obvious equivalence between flat $\lambda$-connection and flat 1 -connection, we argue only the case $\lambda=1$, ie ordinary flat connections.

Let $\mathcal{C}_{1}^{\text {sat }}(X, D)$ denote the category of saturated regular filtered flat sheaves on $(X, D)$. We explain in Section 6.2.1 the construction of a functor $\Phi: \widetilde{\mathcal{C}}(X, D) \longrightarrow \mathcal{C}_{1}^{\text {sat }}(X, D)$. Since it is given by Simpson in [18] essentially in the curve case, we give only an outline. In Section 6.2.2, we will check that it gives an equivalence of the categories. Then we will check that it preserves the characteristic numbers in Sections 6.2.3-6.2.4. which implies that the $\mu_{L}$-stability condition is also preserved.

6.2.1 Construction of $\Phi$ First, we give a construction of $\Phi$. Let $\mathcal{L}_{*}$ be a filtered local system on $(X, D)$. Let $(E, \nabla)$ be the corresponding flat bundle on $X-D$. We have the Deligne extension $(\widetilde{E}, \nabla)$ on $(X, D)$. We put $\mathbf{E}:=\widetilde{E} \otimes \mathcal{O}(* D)$. We have only to give a way of the construction of the $\mathcal{O}_{X}$-coherent submodules ${ }_{\mathrm{a}} E \subset \mathbf{E}$ such that $\nabla_{\mathbf{a}} E \subset{ }_{\mathbf{a}} E \otimes \Omega^{1,0}(\log D)$ and $\bigcup_{\mathbf{a} \in \mathbf{R}^{S} \mathbf{a}} E=\mathbf{E}$.

Let us consider the case $X=\Delta^{n}=\left\{\left(z_{1}, \ldots, z_{n}\right)|| z_{i} \mid<1\right\}$ and $D=\left\{z_{1}=0\right\}$. Then the construction is essentially the same as that given by Simpson [18] in the case $\operatorname{dim} X=1$ We briefly recall it. Let $H(\mathcal{L})$ denote the space of the multivalued flat sections of $\mathcal{L}$. We have the induced filtration $\mathcal{F} H(\mathcal{L})$ and the generalized eigen decomposition $H(\mathcal{L})=\bigoplus_{\omega} \mathbb{E}_{\omega}(H(\mathcal{L}))$, which are compatible in the sense $\mathcal{F}_{a}=\bigoplus_{\omega} \mathcal{F}_{a} \cap \mathbb{E}_{\omega}$. Let $\mathbf{u}=\left(u_{1}, \ldots, u_{r}\right)$ be a frame of $H(\mathcal{L})$, compatible with $(\mathcal{F}, \mathbb{E})$. Then for each $u_{i}$, the numbers $\omega\left(u_{i}\right) \in \mathbf{C}^{*}$ and $a\left(u_{i}\right) \in \mathbf{R}$ are determined by $u_{i} \in \mathbb{E}_{\omega\left(u_{i}\right)}$ and $u_{i} \in \mathcal{F}_{a\left(u_{i}\right)}-\mathcal{F}_{<a\left(u_{i}\right)}$. The complex number $\alpha\left(u_{i}\right)$ is determined by the conditions $\exp \left(-2 \pi \alpha\left(u_{i}\right)\right)=\omega\left(u_{i}\right)$ and $0 \leq \operatorname{Re} \alpha\left(u_{i}\right)<1$. Let $M^{u}$ denote the endomorphism of $H(\mathcal{L})$ or $\mathcal{L}$, which is the unipotent part of the monodromy around $D$, and we put $N:=-(2 \pi \sqrt{-1})^{-1} \log M^{u}$. We regard $u_{i}$ as a multivalued $C^{\infty}$-section of $E$. Then it is standard that $v_{i}:=\exp \left(\log z_{1}\left(\alpha\left(u_{i}\right)+N\right)\right) \cdot u_{i}$ gives a holomorphic section of $E$. Moreover, $\mathbf{v}=\left(v_{1}, \ldots, v_{r}\right)$ gives a frame of the Deligne extension $\widetilde{E}$. For any real number $b$, we put

$$
n\left(b, u_{i}\right):=\max \left\{n \in \mathbf{Z} \mid a\left(u_{i}\right)-\operatorname{Re} \alpha\left(u_{i}\right)+n \leq b\right\}, \quad v_{i}(b):=z_{1}^{-n\left(b, u_{i}\right)} \cdot v_{i} .
$$

Let ${ }_{b} E$ denote the $\mathcal{O}_{X}$-submodule of $\mathbf{E}$ generated by $v_{1}(b), \ldots, v_{r}(b)$. It is easy to check that ${ }_{b} E$ is locally free and independent of a choice of $\mathbf{u}$. It is also easy to see $\mathbf{E}=\bigcup_{b \in \mathbf{R}} b E$. Thus, we obtain the filtration in the case $X=\Delta^{n}$ and $D=\left\{z_{1}=0\right\}$. 
It can be checked that the filtration is independent of a choice of the coordinate $\left(z_{1}, z_{2}, \ldots, z_{n}\right)$ satisfying $D=\left\{z_{1}=0\right\}$.

Let us consider the general case. For any $\mathbf{b} \in \mathbf{R}^{S}$, we have already obtained ${ }_{\mathbf{b}} E$ on $X-D^{[2]}$. The subsheaves ${ }_{\mathbf{b}} E$ of $\mathbf{E}$ are determined by the condition (3).

Lemma 6.1 ${ }_{b} E$ is a coherent $\mathcal{O}_{X}-$ module. Hence, we obtain the saturated regular filtered flat sheaf $\left(\mathbf{E}_{*}, \nabla\right)$ on $(X, D)$.

Proof We may assume that $X=\Delta^{n}$ and $D=\bigcup_{i=1}^{\ell}\left\{z_{i}=0\right\}$. Let $H(\mathcal{L})$ denote the space of multivalued flat sections of $\mathcal{L}$. We have the monodromy endomorphisms $M_{i}(i=1, \ldots, \ell)$ along the loop around $D_{i}$ in the counter clockwise direction. They induce the decomposition

$$
H(\mathcal{L})=\bigoplus_{\omega \in\left(\mathbf{C}^{*}\right)^{\ell}} \mathbb{E}_{\boldsymbol{\omega}} H(\mathcal{L}),
$$

where each $\mathbb{E}_{\omega} H(\mathcal{L})$ is preserved by $M_{i}(i=1, \ldots, \ell)$, and the eigenvalues of $M_{i}$ on $\mathbb{E}_{\omega} H(\mathcal{L})$ are $\omega_{i}$. We also have the filtrations ${ }^{i} \mathcal{F}(i=1, \ldots, \ell)$ of $H(\mathcal{L})$, corresponding to the divisor $D_{i}$. Each ${ }^{i} \mathcal{F}$ is compatible with the decomposition (66). Fix $j$ such that $1 \leq j \leq \ell$. We take a frame $\mathbf{u}=\left(u_{1}, \ldots, u_{r}\right)$ of $H(\mathcal{L})$ compatible with the filtration ${ }^{j} \mathcal{F}$ and the decomposition (66). For each $u_{p}$, the tuple $\omega\left(u_{p}\right) \in$ $\left(\mathbf{C}^{*}\right)^{\ell}$ is determined by $u_{p} \in \mathbb{E}_{\omega}$. Let $\alpha_{i}\left(u_{p}\right) \in \mathbf{C}(i=1, \ldots, \ell)$ be determined by $\exp \left(-2 \pi \alpha_{i}\left(u_{p}\right)\right)=\omega_{i}\left(u_{p}\right)$ and $0 \leq \operatorname{Re} \alpha_{i}\left(u_{p}\right)<1$. We also have the numbers $a_{j}\left(u_{p}\right) \in \mathbf{R}$ such that $u_{p} \in{ }^{j} \mathcal{F}_{a_{j}\left(u_{p}\right)}-{ }^{j} \mathcal{F}_{<a_{j}\left(u_{p}\right)}$. We put

$$
n\left(b_{j}, u_{p}\right):=\max \left\{n \in \mathbf{Z} \mid a_{j}\left(u_{p}\right)-\operatorname{Re} \alpha_{j}\left(u_{p}\right)+n \leq b_{j}\right\} .
$$

Let $N_{i}:=-(2 \pi \sqrt{-1})^{-1} \log M^{u}(i=1, \ldots, \ell)$, where $N_{i}$ denotes the logarithm of the unipotent part of $M_{i}$. We take a sufficiently large integer $I$. Then we put

$$
v_{p}:=z_{j}^{n\left(b_{j}, u_{p}\right)} \cdot \prod_{i \neq j} z_{i}^{I} \prod_{i=1}^{\ell} \exp \left(\log z_{i} \cdot\left(\alpha_{i}\left(u_{p}\right)+N_{i}\right)\right) \cdot u_{p} .
$$

If $I$ is sufficiently large, $v_{p}$ gives the section of ${ }_{\mathbf{b}} E$ on $X$. By the correspondence, we obtain the following morphism, for $j=1, \ldots, \ell$ :

$$
\Phi_{j}: \bigoplus_{p=1}^{r} \mathcal{O}_{X} \cdot v_{p} \longrightarrow_{\mathbf{b}} E
$$

The morphisms $\Phi_{j}(j=1, \ldots, \ell)$ induce the morphism $\Phi: \mathcal{O}^{\oplus \ell \cdot r} \longrightarrow_{\mathbf{b}} E$. The image of $\Phi$ is $\mathcal{O}_{X}$-coherent, and it is the same as ${ }_{\mathbf{b}} E$ on $X-D^{[2]}$. Then it is easy to show that ${ }_{\mathbf{b}} E$ is the same as the double dual of the image of $\Phi$ which is $\mathcal{O}_{X}$-coherent. 
Let $f: \mathcal{L}_{1 *} \longrightarrow \mathcal{L}_{2 *}$ be a morphism. Let $\left(\mathbf{E}_{i *}, \nabla_{i}\right):=\Phi\left(\mathcal{L}_{i}\right)$. We have the induced map $\tilde{f}: \mathbf{E}_{1} \longrightarrow \mathbf{E}_{2}$. It is easy to see that ${ }_{\mathbf{c}} E_{1 \mid X-D^{[2]}} \longrightarrow{ }_{\mathrm{c}} E_{2 \mid X-D^{[2]}}$ is induced. Due to saturatedness of $\left(\mathbf{E}_{2 *}, \nabla\right)$, we obtain maps ${ }_{\mathbf{c}} E_{1} \longrightarrow_{\mathbf{c}} E_{2}$, and thus $\Phi(f):\left(\mathbf{E}_{1 *}, \nabla_{1}\right) \longrightarrow\left(\mathbf{E}_{2 *}, \nabla_{2}\right)$.

6.2.2 Equivalence Let us show that $\Phi$ is gives an equivalence of the categories. To begin with, we consider the case $X=\Delta^{n}$ and $D=\left\{z_{1}=0\right\}$. Let $\mathcal{C}_{1}^{v b}(X, D)$ denote the category of regular filtered flat bundles on $(X, D)$, which is the subcategory of $\mathcal{C}_{1}^{\text {sat }}(X, D)$. By the construction, the image of $\Phi$ is contained in $\mathcal{C}_{1}^{v b}(X, D)$. The following lemma can be shown as in [18].

Lemma 6.2 The functor $\Phi$ gives the equivalence of $\widetilde{\mathcal{C}_{1}}(X, D)$ and $\mathcal{C}_{1}^{v b}(X, D)$. It is also compatible with direct sums, duals, and tensor products.

Lemma 6.3 In the case $X=\Delta^{n}$ and $D=\left\{z_{1}=0\right\}$, we have $\mathcal{C}_{1}^{v b}(X, D) \simeq \mathcal{C}_{1}^{\text {sat }}(X, D)$ naturally. In particular, $\Phi$ gives the equivalence $\tilde{\mathcal{C}}_{1}(X, D) \simeq \mathcal{C}_{1}^{\text {sat }}(X, D)$.

Proof Let $\left(\mathbf{E}_{*}, \nabla\right)$ be a saturated regular filtered flat sheaf on $(X, D)$. We put $(E, \nabla):=\left(\mathbf{E}_{*}, \nabla\right)_{\mid X-D}$, and let $\mathcal{L}$ denote the underlying local system on $X-D$. Let $H(\mathcal{L})$ denote the space of multivalued flat sections of $\mathcal{L}$.

Recall that there exists a subset $W \subset D$ with $\operatorname{codim}_{X}(W) \geq 3$ such that $\left(\mathbf{E}_{*}, \nabla\right)_{\mid X-W}$ is a regular filtered flat bundle on $(X-W, D-W)$ (Lemma 2.9). Let $P$ be any point of $D-W$, and let $\left(U_{P}, z_{1}, \ldots, z_{n}\right)$ be a holomorphic coordinate neighbourhood such that $z_{1}^{-1}(0)=U_{P} \cap D$ and $U_{P} \cap W=\varnothing$. Due to Lemma 6.2, we have the unique filtration $\mathcal{F}$ of $H\left(\mathcal{L}_{\mid U_{P} \backslash D}\right) \simeq H(\mathcal{L})$ corresponding to $\left(\mathbf{E}_{*}, \nabla\right)_{\mid U_{P}}$. Due to the uniqueness, it is independent of the choice of $P$ and $U_{P}$.

Let $\mathbf{u}=\left(u_{1}, \ldots, u_{r}\right)$ be a frame of $H(\mathcal{L})$ compatible with the filtration $\mathcal{F}$ and the generalized eigen decomposition with respect to the monodromy around $D$. For any real number $b \in \mathbf{R}$, we construct $\mathbf{v}(b)=\left(v_{1}(b), \ldots, v_{r}(b)\right)$ as above. Then for any $P \in D-W, \mathbf{v}(b)$ gives a holomorphic frame of ${ }_{b} E_{\mid U_{P}}$ compatible with the filtration due to Lemma 6.3. Hence, each $v_{i}(b)$ gives a section of ${ }_{b} E_{\mid X-W}$. Due to the saturatedness of $\left(\mathbf{E}_{*}, \nabla\right), v_{i}(b)$ gives a section of ${ }_{b} E$ on $X$. It is easy to see that $\mathbf{v}(b)$ gives a frame of ${ }_{b} E$, and in particular, ${ }_{b} E$ is locally free. Hence, $\left(\mathbf{E}_{*}, \nabla\right)$ is a regular filtered flat bundle on $(X, D)$.

It is easy to see that $\Phi$ is equivalent for general $(X, D)$. Let us see the fully faithfulness of $\Phi$. The faithfulness is obvious. Let $f: \Phi\left(\mathcal{L}_{1 *}\right) \longrightarrow \Phi\left(\mathcal{L}_{2 *}\right)$ be a morphism in $\mathcal{C}_{1}^{\text {sat }}(X, D)$. We have the map $g: \mathcal{L}_{1} \longrightarrow \mathcal{L}_{2}$ corresponding to $f$. We would like to 
check that $g$ preserves the filtrations ${ }^{i} \mathcal{F}$. Let $P$ be any point of $D_{i}^{\circ}$, and $\left(U, z_{1}, \ldots, z_{n}\right)$ be any coordinate neighbourhood such that $U \cap D=z_{1}^{-1}(0)$. Applying Lemma 6.3, we obtain that $g$ preserves the filtration ${ }^{i} \mathcal{F}$ on $U \backslash D_{i}$. Thus, we obtain the fully faithfulness.

Let us show the essential surjectivity. Let $\left(\mathbf{E}_{*}, \nabla\right)$ be a saturated filtered flat sheaf on $(X, D)$. Let $\mathcal{L}$ denote the local system corresponding to $\left(\mathbf{E}_{*}, \nabla\right)_{\mid X-D}$. We have only to construct the appropriate filtrations ${ }^{i} \mathcal{F}$ of $\mathcal{L}_{\mid U_{i} \backslash D}$ on appropriate neighbourhoods of $D_{i}$. Let $P$ be any point of $D_{i}^{\circ}$, and $\left(U_{P}, z_{1}, \ldots, z_{n}\right)$ denote any coordinate neighbourhood around $P$ such that $z_{1}^{-1}(0)=U_{P} \cap D$. Due to Lemma 6.2, we obtain the unique filtration ${ }^{i} \mathcal{F}$ of $\mathcal{L}_{\mid U_{P} \backslash D}$. We obtain the filtration ${ }^{i} \mathcal{F}$ on $\bigcup_{P \in D_{i}^{\circ}} U_{P}$ by gluing them, due to the uniqueness. Thus, we obtain that $\Phi$ is essentially surjective, and hence equivalent.

6.2.3 The parabolic first Chern class We have the $\mathbf{Z}$-action on $\mathbf{R} \times \mathbf{C}$ given by $n \cdot(a, \alpha)=(a+n, \alpha-n)$. It induces the action of $\mathbf{Z}$ on $\mathcal{K} \mathcal{M S}\left(\mathbf{E}_{*}, i\right)$. The following lemma is clear from the construction of $\Phi$.

Lemma 6.4 We have the bijective correspondence of the sets $\mathcal{K} \mathcal{M S}\left(\Phi\left(\mathcal{L}_{*}\right), i\right) / \mathbf{Z}$ and $\mathcal{K} \mathcal{M S}\left(\mathcal{L}_{*}, i\right)$, which is given by $(a, \alpha) \longmapsto(b, \omega)=(a+\operatorname{Re} \alpha, \exp (-2 \pi \sqrt{-1} \alpha))$ for $(a, \alpha) \in \mathcal{K} \mathcal{M S}\left(\Phi\left(\mathcal{L}_{*}\right), i\right)$. Moreover, $\operatorname{rank}^{i} \operatorname{Gr}_{(a, \alpha)}^{F, \mathbb{E}}=\operatorname{rank}^{i} \operatorname{Gr}_{(b, \omega)}^{\mathcal{F}, \mathbb{E}}$.

Corollary 6.5 $\Phi$ preserves the parabolic first Chern classes, ie,

$$
\operatorname{par}-\mathrm{c}_{1}\left(\mathcal{L}_{*}\right)=\operatorname{par}_{-1}\left(\Phi\left(\mathcal{L}_{*}\right)\right) .
$$

In particular, when $X$ is a smooth irreducible projective variety with an ample line bundle $L$, the $\mu_{L}$-stability of $\mathcal{L}_{*}$ and $\mu_{L}$-stability of $\Phi\left(\mathcal{L}_{*}\right)$ are equivalent.

Proof Recall Lemma 3.23. It is shown for the case in which $\left(\mathbf{E}_{*}, \nabla\right)$ is graded semisimple and $X$ is two dimensional. However, the graded semisimplicity condition is not necessary as is explained in Remark 3.21. The assumption $\operatorname{dim} X=2$ is also not necessary, due to the Lefschetz theorem. Then the claim of the corollary follows from Lemma 3.23 and the correspondence of the KMS-spectrums given in Lemma 6.4. $\square$

\subsubsection{The second parabolic Chern character}

Lemma 6.6 Let $X=\Delta^{n}=\left\{\left(z_{1}, \ldots, z_{n}\right)|| z_{i} \mid<1\right\}$, and $D=D_{1} \cup D_{2}$, where $D_{i}=\left\{z_{i}=0\right\}$. Let $\left(\mathbf{E}_{*}, \nabla\right)$ be a saturated regular filtered flat sheaf on $(X, D)$.

(1) $\left(\mathbf{E}_{*}, \nabla\right)$ is a regular filtered flat bundle on $(X, D)$. 
(2) Let $\mathbf{c}$ be any element of $\mathbf{R}^{2}$, and let $\mathbf{c} E$ denote the $\mathbf{c}$-truncation. Let $\mathcal{L}_{*}$ be the corresponding filtered local system on $(X, D)$. Then we have the equality:

$$
\operatorname{rank}^{\stackrel{2}{2}} \operatorname{Gr}_{(\mathbf{b}, \boldsymbol{\omega})}^{\mathcal{F}, \mathbb{E}}(\mathcal{L})=\operatorname{rank}^{\stackrel{2}{G}} \operatorname{Gr}_{(\mathbf{a}, \boldsymbol{\alpha})}^{F, \mathbb{E}}(\mathbf{c} E)
$$

Here the meaning of the notation is as follows:

- $\mathbf{b}=\left(b_{1}, b_{2}\right)$ and $\boldsymbol{\omega}=\left(\omega_{1}, \omega_{2}\right)$ denote elements of $\mathbf{R}^{2}$ and $\left(\mathbf{C}^{*}\right)^{2}$ respectively.

- $\mathbf{a}=\left(a_{1}, a_{2}\right)$ and $\boldsymbol{\alpha}=\left(\alpha_{1}, \alpha_{2}\right)$ denote elements of $\mathbf{R}^{2}$ and $\mathbf{C}^{2}$ respectively, determined by the conditions $c_{i}-1<a_{i} \leq c_{i}$, $\exp \left(-2 \pi \sqrt{-1} \alpha_{i}\right)=\omega_{i}$ and $a_{i}+\operatorname{Re} \alpha_{i}=b_{i}$.

Proof Let $\mathcal{L}_{*}=\left(\mathcal{L},{ }^{1} \mathcal{F},{ }^{2} \mathcal{F}\right)$ be as above. Let $\mathbf{u}$ be a frame of $H(\mathcal{L})$ compatible with the filtrations ${ }^{k_{\mathcal{F}}}(k=1,2)$ and the generalized eigen decompositions of $H(\mathcal{L})$. For each $u_{j}$ and the divisor $D_{k}$, the numbers $\alpha_{k}\left(u_{j}\right)$ and $a_{k}\left(u_{j}\right)$ are determined as before. For the monodromies around $D_{k}$, we obtain the nilpotent endomorphism $N_{k}$ as before. The holomorphic section $v_{j}$ is given by $v_{j}:=\exp \left(\sum \log z_{k}\left(\alpha_{k}\left(u_{j}\right)+N_{k}\right)\right)$. Let $n_{k}\left(u_{j}\right)$ be the numbers determined by the condition $c_{k}-1<n_{k}\left(u_{j}\right)+a_{k}\left(u_{j}\right)-$ $\operatorname{Re} \alpha_{k}\left(u_{j}\right) \leq c_{k}$. We put

$$
\tilde{v}_{j}:=\prod_{k=1,2} z_{k}^{-n_{k}\left(u_{j}\right)} \cdot v_{j}
$$

Then $\widetilde{\mathbf{v}}=\left(\widetilde{v}_{1}, \ldots, \widetilde{v}_{r}\right)$ gives the frame of $\mathbf{c} E_{\mid X-\left(D_{1} \cap D_{2}\right)}$. Due to the saturatedness, $\widetilde{\mathbf{v}}=\left(\widetilde{v}_{1}, \ldots, \widetilde{v}_{r}\right)$ gives the frame of $\mathbf{c} E$, and hence $\mathbf{c} E$ are locally free. Thus, the first claim is proved. The frame $\widetilde{\mathbf{v}}$ is compatible with ${ }^{i} \mathbb{E}$ and ${ }^{i} F$, and we have ${ }^{k} \operatorname{deg}{ }^{F}\left(\widetilde{v}_{j}\right)=$ $a_{k}\left(u_{j}\right)-\operatorname{Re} \alpha_{k}\left(u_{j}\right)+n_{k}\left(u_{j}\right)$ and $\widetilde{v}_{j \mid D_{k}} \in{ }^{k} \mathbb{E}\left(\alpha_{k}\left(u_{j}\right)-n_{k}\left(u_{j}\right)\right)$. Thus, the second claim follows.

Let $X$ be a smooth irreducible complex projective variety with an ample line bundle $L$, and let $D$ be a simple normal crossing hypersurface.

Corollary 6.7 Let $\left(\mathbf{E}_{*}, \nabla\right)$ be a saturated regular filtered flat sheaf on $(X, D)$, and let $\mathcal{L}_{*}$ denotes the corresponding filtered local system. Then the following holds:

$$
\int_{X} \operatorname{par}^{-\mathrm{ch}_{2, L}}\left(\mathcal{L}_{*}\right)=\int_{X} \operatorname{par}^{-\mathrm{ch}_{2, L}}\left(\mathbf{E}_{*}\right)
$$

Corollary 6.8 Let $\mathcal{L}_{*}$ be a $\mu_{L}$-stable filtered local system on $(X, D)$. Then the Bogomolov-Gieseker inequality for $\mathcal{L}_{*}$ holds:

$$
\int_{X} \operatorname{par-\operatorname {ch}_{2,L}}\left(\mathcal{L}_{*}\right) \leq \frac{\int_{X} \operatorname{par}^{2}{ }_{1, L}^{2}\left(\mathcal{L}_{*}\right)}{2 \operatorname{rank} \mathcal{L}} .
$$


Proof Recall that saturated regular filtered flat shaves are regular filtered flat bundles in codimension two (Lemma 2.9). Hence, the claim follows from Corollary 6.5, Corollary 6.7 and Corollary 3.20.

Corollary 6.9 Let $\mathcal{C}_{1}^{\text {poly }}$ be the category of $\mu_{L}$-polystable regular filtered flat bundles on $(X, D)$ with trivial characteristic numbers, and let $\widetilde{\mathcal{C}}_{1}^{\text {poly }}$ be the category of $\mu_{L}-$ polystable filtered local systems on $(X, D)$ with trivial characteristic numbers. Then the functor $\Phi$ naturally induces an equivalence of these categories.

Proof We have only to remark that saturated $\mu_{L}$-stable regular filtered flat sheaves with trivial characteristic numbers are regular filtered bundles (Theorem 5.17).

Remark 6.10 By a result in [15] and the existence of a pluri-harmonic metric for $\Phi\left(\mathcal{L}_{*}\right)$, the filtrations ${ }^{i} \mathcal{F}$ for $\mu_{L}$-stable filtered local systems $\mathcal{L}_{*}$ with trivial characteristic numbers satisfy some compatibility around the intersection points of $D$.

\section{References}

[1] O Biquard, Fibrés de Higgs et connexions intégrables: le cas logarithmique (diviseur lisse), Ann. Sci. École Norm. Sup. (4) 30 (1997) 41-96 MR1422313

[2] K Corlette, Flat G-bundles with canonical metrics, J. Differential Geom. 28 (1988) 361-382 MR965220

[3] P Deligne, Équations différentielles à points singuliers réguliers, Lecture Notes in Math. 163, Springer, Berlin (1970) MR0417174

[4] S K Donaldson, Anti self-dual Yang-Mills connections over complex algebraic surfaces and stable vector bundles, Proc. London Math. Soc. (3) 50 (1985) 1-26 MR765366

[5] S K Donaldson, Infinite determinants, stable bundles and curvature, Duke Math. J. 54 (1987) 231-247 MR885784

[6] J Eells, Jr, J H Sampson, Harmonic mappings of Riemannian manifolds, Amer. J. Math. 86 (1964) 109-160 MR0164306

[7] C Hertling, C Sevenheck, Limits of families of Brieskorn lattices and compactified classifying spaces arXiv:0805.4777

[8] R Hotta, K Takeuchi, T Tanisaki, D-modules, perverse sheaves, and representation theory, Progress in Math. 236, Birkhäuser, Boston (2008) MR2357361Translated from the 1995 Japanese edition by Takeuchi

[9] J N N Iyer, C T Simpson, A relation between the parabolic Chern characters of the de Rham bundles, Math. Ann. 338 (2007) 347-383 MR2302066 
[10] J Jost, K Zuo, Harmonic maps of infinite energy and rigidity results for representations of fundamental groups of quasiprojective varieties, J. Differential Geom. 47 (1997) 469-503 MR1617644

[11] M Lübke, A Teleman, The universal Kobayashi-Hitchin correspondence on Hermitian manifolds, Mem. Amer. Math. Soc. 863 (2006) MR2254074

[12] V B Mehta, A Ramanathan, Restriction of stable sheaves and representations of the fundamental group, Invent. Math. 77 (1984) 163-172 MR751136

[13] T Mochizuki, Wild harmonic bundles and wild pure twistor D-modules arXiv: 0803.1344

[14] T Mochizuki, Kobayashi-Hitchin correspondence for tame harmonic bundles and an application, Astérisque 309 (2006) MR2310103

[15] T Mochizuki, Asymptotic behaviour of tame harmonic bundles and an application to pure twistor D-modules. II, Mem. Amer. Math. Soc. 185 (2007) xii+565 MR2283665

[16] C Sabbah, Polarizable twistor D-modules, Astérisque 300 (2005) MR2156523

[17] C T Simpson, Constructing variations of Hodge structure using Yang-Mills theory and applications to uniformization, J. Amer. Math. Soc. 1 (1988) 867-918 MR944577

[18] C T Simpson, Harmonic bundles on noncompact curves, J. Amer. Math. Soc. 3 (1990) 713-770 MR1040197

[19] C T Simpson, Higgs bundles and local systems, Inst. Hautes Études Sci. Publ. Math. (1992) 5-95 MR1179076

[20] C T Simpson, The Hodge filtration on nonabelian cohomology, from: "Algebraic geometry—Santa Cruz 1995”, Proc. Sympos. Pure Math. 62, Amer. Math. Soc. (1997) 217-281 MR1492538

[21] C T Simpson, The construction problem in Kähler geometry, from: "Different faces of geometry", Int. Math. Ser. (N. Y.) 3, Kluwer/Plenum, New York (2004) 365-402 MR2103668

[22] K Uhlenbeck, S-T Yau, On the existence of Hermitian-Yang-Mills connections in stable vector bundles, Comm. Pure Appl. Math. 39 (1986) S257-S293 MR861491Frontiers of the mathematical sciences: 1985 (New York, 1985)

Research Institute for Mathematical Sciences, Kyoto University Kyoto 606-8502, Japan takuro@kurims.kyoto-u.ac.jp

Proposed: Simon Donaldson

Seconded: Tom Mrowka, Ron Fintushel
Received: 11 February 2008 Revised: 26 September 2008 